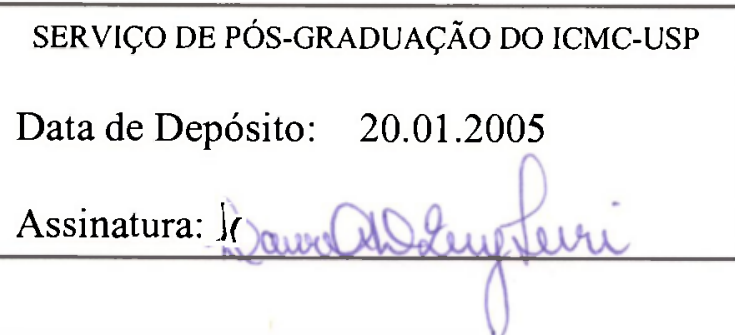

\title{
Multiplicidades e multiplicidades mixtas de ideais m-primários
}

Márcio Alexandre de Oliveira Reis

Orientador: Prof. Dr. Victor Hugo Jorge Pérez

Dissertação apresentada ao Instituto de Ciências Matemáticas e de Computação - ICMC-USP, como parte dos requisitos para obtenção do título de Mestre em Matemática.

USP - São Carlos

Janeiro/2005 
Aluno: Marcio Alexandre de Oliveira Reis

A Comissão Julgadora:

Prof. Dr. Victor Hugo Jorge Pérez

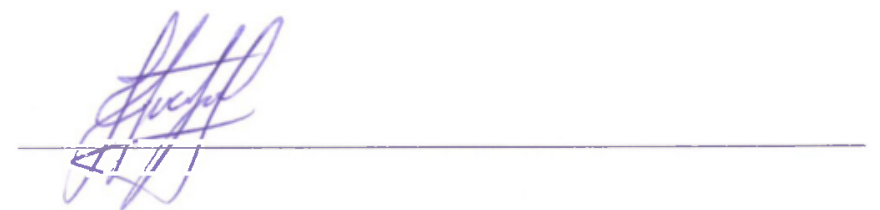

Prof. Dr. Daniel Levcovitz

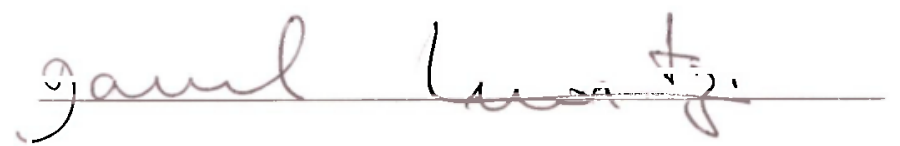

Prof. Dr. João Nivaldo Tomazella

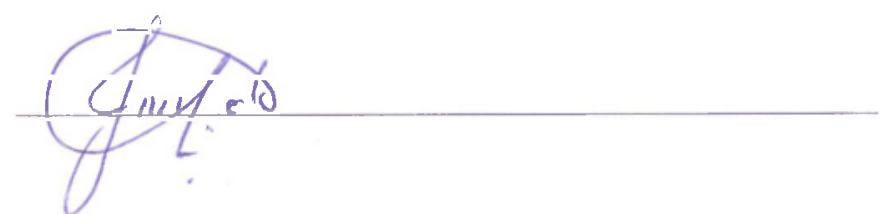




\section{AGRADECIMENTOS}

A minha família, especialmente aos meus pais, Florêncio Reis que nunca se conformon por eu ter parado de estudar, e Zilda de Oliveira Reis por ter sido uma mãe zclosa com seus filhos.

Ao meu primo Cláudio de Oliveira, por ter conseguido um emprego para mim em Montenegro, sem o qual talvez eu jamais tivesse voltado às salas de aulas, depois de sete longos anos.

À memória do meu chefe, Ercílio Leindorff, por ter permitido que eu me ausentasse do trabalho, em alguns momentos, para terminar o Ensino Médio e por seus incentivos para que eu fizesse um curso superior.

Aos meus colegas do "Barraco"em Montencgro, os molhores amigos que tive.

Ao Prof. Dr. Pencireiro, por ter acreditado em mim.

Ao Prof. Dr. Osmar Giuliani pela bolsa e pelo incentivo tăo necessários.

A todos os professores da LFSM, por terem me suportado, em especial a Profa. Dr. Maria de Lourdes.

Aos meus amigos da LFSM, principalmente ao Marcio Paim e ao Fabiano Becker.

Ao Prof. 1)r. Victor Hugo Jorge Pŕre\%, pelo profissionalismo, seriedade e paciencia demonsirados durante a realização deste trabalho.

A minha esposa, Cristina Spohr, pelo companheirismo, paciència e o apoio, que foram fundamentais desde que a conheci. Também a Deus, por ter me presenteado com uma pessoa tão maravilhosa cono cla e por ter me dado tantas oportunidades nesta vida. que por muitas vezes cu arho năo ter merecido.

Ao Thiago de Melo pela ajuda com o $B \mathrm{~T}_{\mathrm{E}} \mathrm{X}$.

Aos funcionários da LSP, pelo profissionalismo, competência e pela simpatia que 
demonstram no desenvolver de suas tarefas.

À CAPES pela bolsa.

E a mim por ter largado a Biologia e mesmo tendo desistido várias vezes da Matemática (foram três vezes), ter voltado a trás. 


\section{RESUMO}

Neste trabalho, estudamos as principais propriedades da teoria geral da multiplicidade algébrica de um ideal $I$ de um anel $A$, com relação a um $A$-módulo $M$. A definição da multiplicidade surge a partir do conceito de comprimento e a partir disto estudamos as relaçôes entre o ś́mbolo da multiplicidade o o comprimento. Támbém estudamos a função de Hilbert associada a vários idteais $\mathcal{M}$-primários o definimos as multiplicidades mixtas, definidas originalmente por B. Teissier e J.J. Risler. Utilizando as propriedades da multiplicidades algébrica, calculamos o número de Milnor de algumas hipersuperfícies complexas com singularidade isolada. 


\section{ABSTRACT}

In this work, we study the main properties of general theory of algebraic multiplicities of an ideal of a ring $A$, with respect to $A$-module $M$. The definition of multiplicities arises ont from concept of length and from this concept we study the relations among the multiplicity's symbol and the length. We also study the Hilbert's functions associated to many ideals $\mathcal{M}$-primary and we define the mixed multiplicitics which was defined originally by B. Teissier and J.J. Risler. Using the propertics of algebraic multiplicities we calculate the Milnor's number of some complex hypersurface with isolated singularity. 


\section{Sumário}

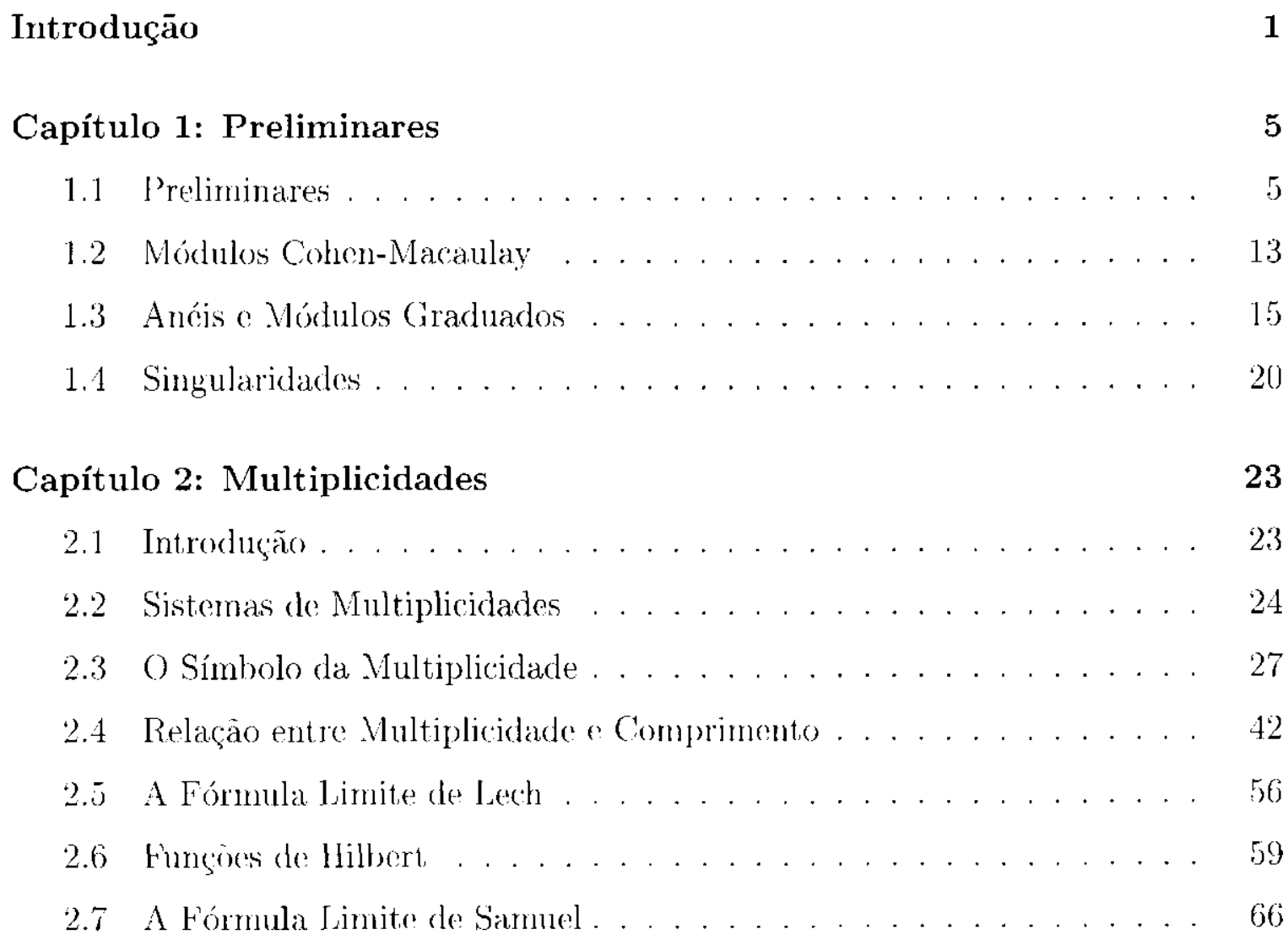

Capítulo 3: Multiplicidades Mixtas de dois Ideais $\mathcal{M}$-primários $\quad 75$

3.1 Introduçäo . . . . . . . . . . . . . . 75

3.2 Resultados Preliminares . . . . . . . . . . . . 76

3.3 Ideais Bihomogeneos . . . . . . . . . . . . . . . . . 81

3.4 A Função de Ifilbert de dois Ideais $\mathcal{M}$-primários . . . . . . . . . 88

3.5 Multiplicidade Mixta e Dependência Integral . . . . . . . . . 95 
Capítulo 4: Multiplicidades Mixtas $\quad 103$

4.1 Introduçăo . . . . . . . . . . . . . . . . . . . . . . . . . 103

4.2 Resultados Básicos . . . . . . . . . . . . . . . . . . . 103

4.3 A Definiçäo de Multiplicidade Mixta . . . . . . . . . . . . . 107

Capítulo 5: Aplicações $\quad 115$

5.1 O Número de Milnor . . . . . . . . . . . . . . . . . . . . 115

5.2 o Cálculo do Númcro de Milnor . . . . . . . . . . . . . . 117

5.3 Multiplicidades Mixtas e o Número de Milnor . . . . . . . . . . . . 119

Apêndice A: Localização $\quad 123$

A.1 Localização e Multiplicidades . . . . . . . . . . . . . . . . 123

Apêndice B: Teorema de Rees $\quad 127$

B.1 O Complexo de Koszul . . . . . . . . . . . . . . . . 127

B.2 Complexo de Košul e Multiplicidades Mixtas . . . . . . . . . 135

Referências Bibliográficas $\quad 145$ 


\section{Introdução}

Fim [20], Sarnuel provou que se $I$ é um ideal $\mathcal{M}$-primário de uma anel local Noetheriano $(A, \mathcal{M})$, entào para valores suficientemente grandes de $n$, a função $H: \mathbb{N}^{2} \longrightarrow \mathbb{N}$ definida por

$$
H(n)-L_{A}\left(A / I^{n}\right)
$$

é um polinomio em $n$, onde $L_{A}\left(A / I^{n}\right)$ denota o comprimento do anel $A / I^{n}$. O fato mais importante neste resultado, é que o coeficiente de maior grau deste polinomio, é igual a $\frac{e(I \mid A)}{d !}$, onde e $(I \mid A)$ ó um número inteciro o qual é chamado a multiplicidade de Hilbert-Samuel do ideal $I$ em A. A partir deste artigo, vários matemáticos passaram a investigar as propriedades deste núnero, entre eles podemos citar C. Lech, D. Northcott, D. Rees, etc. Mais tarde, Teissier e Risler mostraram que se $I_{1} \ldots, I_{s}$ é um conjunto finito de ideais $\mathcal{M}$-primários do anel Noetheriano local $(A, \mathcal{M})$, entäo a função $I I: \mathbb{N}^{2} \longrightarrow \mathbb{N}$, definidla por

$$
H\left(n_{1}, \ldots, n_{s}\right)=L_{\lambda}\left(M /\left(I_{1}^{n_{1}} \ldots I_{s}^{n_{s}}\right)\right)
$$

também é um polinômio en $n_{1}, \ldots, n_{s}$, rescle que estes inteiros positivos fossem suficientemente grandes. Além disso, os coeficientes do termo de maior gran deste. polinômio foram chamados de multiplicidades mixtas. A contribuiçăo de Teissier e Risler, foi a de mostrar que estes coelicientes, eram na verdade, a multiplicidade de Hilbert-Samuel de um ideal gerado por uma sequencia de elementos, os quais eles chamaram de superficiais. Além disso, eles deram uma interpretaçào geométrica para estas multiplicidades.

Nesta dissertação, nós seguimos basicamente o ponto de vista de [32] e [13], para estudar o símbolo da multiplicidade. Durante o estudo do símbolo da multiplicidade, 
cm algumas situaçóes, nos restringimos an caso local para estudar alguns resultados importantes de Álgebra Comutativa, como por exemplo, mostrar que se $M$ é um $A$ módulo Cohen-Macaulay, onde $A$ é anel Noetheriano local e $I$ é un idcal gerado por um sistema de parâmetros, então o comprimento $L_{A}(M / I M)$ e a multiplicidade do ideal $I$ coincidem numericamente, com relação ao módulo $M$

Também é feito um estudo da função de Hilbert do mais do um ideal $\mathcal{M}$-primário e algumas fórmulas clássicas na teoria da multiplicidade, como a Fórmula Limite de Samuel e a Fórmula Limite de Samuel, são estabelecidas nesta dissertação.

Este texto também contém dois apêndices. O primeiro contém vários resultados da teoria da multiplicidade como, por exemplo, o Princípio da Localização e a Lei Associativa. No segundo, utilizamos o complexo de Koszul para demonstrar uma generalização do Teorema de Teissier devida a D. Rees. A justificativa destes apendices está no fato de que se estes resultados fossem acrescentados no corpo da disscrtação, está ficaria muito estensa, por isto, a sua leitura pode ser omitida, ou pode ser lida como cultura matemática. Os resultados dos apêndices não são tão detalhados como aqueles que fazem parte dos cinco capítulos que a compõe e em algumas situações, sua leitura é um pouco difícil.

Procuramos dar as referências sobre praticamente todos os resultados que não foram demonstrados neste texto. Infelizmente, não é possível dar os créditos merecidos a cada autor, pois isto exigiria um trabalho de pesquisa bibliográfico que demandaria muito tempo, o talvez ainda assim nâo teríamos sidos justos.

Apesar de termos corrigido cuidadosamente todos os detalhes das demonstraçooes feitas aqui, temos a certeza de que alguns erros, quer sejam de naturcza gramatical ou matemático, permaneceram. A bibliografia é estensa c não temos a pretensão de que o leitor acredite que as lemos na integra, mas todas clas foram consultadas, em maior ou menor grau para que tivéssemos um visão da t,coria da multiplicidade, bem como sua história, seu desenvolvimento atual e aplicaçôses quer sejam geométricas ou puramente algébricas.

Este trabalho não contém nenhum resultado original, talvez apenas alguns crros ou equívocos matemáticos que eventualmente persistiram, após o trabalho final de correçäo. 
O trabalho está organizado do seguinte modo.

No Capítulo 1, encontram-se os pré-requisitos de Álgebra Comulativa e da Teoria de Singularidades. Alguns exemplos säo apresentados para fixar alguns conceitos deste capítulo. As principais referencias para este capítulo foram basicamente as seguintes: sobre Álgebra Comnlativa [1], [9]: [21], [12] e principalmente [13].

No Capítulo 2. definimos sistemas de multiplicidades e estudamos as principais propriedades do símbolo da multiplicidade, que é definido indutivamente e a relacionamos com o comprimento de um módulo. Também usamos a função de Hilbert para estabelecer a fómula Limite de Samuel. A fómula Limite de Lech também é provada. Neste capítulo utilizamos basicamente [32] e [13].

No Capitulo 3, estudamos a função de Hilbert de dois idedis $\mathcal{M}$-primários num anel Noetheriano local e provamos que ela ó um polinômio em duas variávois. Várias propriedades da multiplicidade são estabelecidas, sendo que um dos principais resultados é que em determinadas situações, a multiplicidade e o fecho integral estão intimamente relacionados, bem como o conceito de redução. Aqui usamos as roferèncias $[3],[11]$ e $[28]$.

No Capítulo 1, mostramos que as multiplicidades mixtas são de fato a multiplicidade de Samuel de: um ideal gerado por uma sequência de elementos superficiais. Também mostramos o Teorema de Teissier e Risler. Convóm observar que segundo alguns autores, o conceito de uma sequência de elementos superficiais é muito "vaga", pois é muito difícil exibir uma sequència constituída intciramente por estes elementos, num caso geral. Neste capítulo utilizamos [23] e [34].

No Capítulo 5, usamos as propriedades do símbolo da multiplicidade para calcular o número de Milnor de uma hipersuperfície complexa analítica com singularidade isolada na origem. Vários exemplos são calculados usando as propricdades do símbolo da multiplicidade estudadas no Capítulo 2. Aqui utilizamos [23] :[24].

No Apèndice A enunciamos, scm demonstração, vários resultados sobre o símbolo da multiplicidade, tais como o P’rincípio da Localizaçào, a Fórmula da Fxtensão e a Lei Associativa. Os resultados deste apendice podem ser encontrados em [32] o [13].

No Apendice. B mostramos que o simbolo da multiplicidade e a caracteristica do Euler-Poincaró são numericamente iguais. Também apresentamos a demonstração do 
Teorema de Teissier, devida a Rees, ntilizando o complexo de Koszul. As referências para este apêndice são [13], [17] e [22]. 


\section{Capítulo 1}

\section{Preliminares}

\section{$1.1 \quad$ Preliminares}

Por um anel $A$, entendemos um anel Noetheriano comutativo com unidade $(1 \neq 0)$. Todo anel $A \neq 0$, possui pelo menos um ideal maximal. No caso de $A$ possuir exatamente un idcal maximal, diremos que $A$ é um anel local. Usaremos $(A, \mathcal{M})$ para denotar o anel local $A$ com o seu ideal maximal $\mathcal{M}$. Se $A$ é um anel local com idcal maximal $\mathcal{M}$, cntão o corpo $k=A / \mathcal{M}$, ć chamado o corpo residual do anel $A$, que supomos ser sempre infinito.

Corpos são exemplos de anéis locais. Também o anel das coordenadas $\mathcal{O}_{X, 0}$ de $K(X)-k\left[x_{1}, \ldots, x_{n}\right] / I$, localizado no ideal maximal $\mathcal{M}=\left(x_{1}, \ldots, x_{n}\right)$, onde $I$ éo icleal que define $X$, é local.

A interseção de todos os idcais maximais do anel $A$ ć um ideal, chamado o radical de Jacobson de $A$, denolado por $\mathcal{R}(A)$. So $(A, \mathcal{M})$ é un anel local, então $\mathcal{R}(A)$ é exatanente seu ideal maximal $\mathcal{M}$.

Definição 1.1.1: Diremos que um A-módulo $M$ é Noetheriano se todo submódulo $N$ de $M$ é finitamente gerado. Como todo anel $A$ é um $A$-módulo sobre si mesmo, diremos que $A$ é um anel Noetheriano se todo ideal $I$ de $A$ é finitamente gerado.

Ná verdade, näo é necessário verificar se todos os idcais de $A$ sä́o finitamente 
gerados, pois se todos os ideais primos de $A$ são finitamente gerados, então $A$ ć Noctheriano ([9], pag. 17).

Mostra-se que as duas afirmaçôes abaixo, são equivalenus em qualquer $A$-módulo Noetheriano $M$ :

i) Vale a condição de cadeia ascendente, isto é, qualquer cadcia ascendente de submódulos de $M$

$$
N_{1} \subset N_{2} \subset \cdots \subset N_{n} \subset \cdots
$$

éstacionária, ou seja, existe um inteiro positivo $n$, tal que $N^{n}=N^{n-r}$, para todo inteiro $r \geq 1$

ii) Qualquer coleção não-vazia de submódulos de $M$, contóm um elemento máximo (com relação à inclusão de conjuntos).

Anćis principais e corpos são exemplos de anćis Noetherianos.

Teorema 1.1.2: (Teorema da Base de Hilbert) ([9], pag. 16) Se $A$ é um anel Noetheriano, então os anćis $A\left[x_{1}, \ldots, x_{n}\right]$ e $A\left\{x_{1}, \ldots, x_{n}\right\}$, dos polinômios e das sérics convergentes sobre $A$, respectivamente, nas indeterminadas $x_{1}, \ldots, x_{n}$, também são Noetherianos.

O Teorema da Base de Hilbert, além de sua importância própria, nos fornece uma mancira concreta de obtermos uma infinidade de exemplos de anéis Noctherianos. Para ver isto, basta tomar um anel Noetheriano o um conjunto finito de indeterminadas sobre o anel $A$ e construir o ancl de polinomios sobre $A$ nestas indeterminadas.

Definição 1.1.3: Um A-módulo $M$ é chamado Artiniano se qualquer cadeia descendente de submódulos

$$
M=M_{0} \supset M_{1} \supset \cdots
$$

ć estacionária. Diremos que um anel A é Artiniano se ele é Artiniano como A-módulo.

Espaços vetoriais de dimensão finita sobre um corpo $k$, são excmplos de $k$-módulos Artinianos. Todo anel Artiniano é Noetheriano ([9], pag. 16), mas a recíproca não é 
válida, por exemplo, o anel $\mathbb{Z}$ é Noetheriano, mas não é Artiniano.

O próximo Teorema reúno várias propriedades sobre anéis Artinianos que usaremos durante este trabalho. A prova destes resultados se encontram em (11], Capítulo $8)$.

Um elemento $b$ do ancl $A$ co chamado nilpotente, se existe um inteiro positivo s (dependendo de $b$ ), tal que $b^{s}=0$. O conjunto de todos os elementos nilpotentes de um anel $A$ é um ideal, chamado de ideal nilradical de $A$ e denotado por nil(A). Um ideal $I$ de um ancl $A$ ć chamado nilpotente, se existe um inteiro positivo $r$ (dependendo de $I$ ), tal que $I^{r}=0$.

Teorema 1.1.4: 1) Um anel $A$ é Artiniano se, esomente se, $A$ ó Voetheriano o $\operatorname{din} A=0$.

2) Num anel Artiniano $A$, o nilradical de $A$ é um ideal nilpotente.

3) Todo ideal primo num anel Artiniano $A$ é maximal. Além disso, num anel Artiniano existe apenas un número finito de ideais maximais.

Uma série normal num A-módulo $M$, é uma cadeia descendente finita de submódulos de $M$ tal que

$$
M=M_{0} \supset M_{1} \supset \cdots \supset M_{l}-\{0\}
$$

com $M_{i} \neq M_{i+1}$, para $i=0, \ldots, l-1$. O número natural $l$ é chamado o comprimento da série normal. A série normal (1.1) é chamadi una série de composição, se $M_{i} / M_{i+1}$ é unn módulo, cujos únicos submódulos são o $\{0\}$ e o próprio $M_{i} / M_{i+1}$, para $i=0, \ldots, l-1$.

Teorema 1.1.5: (i1], pag. 77) Se um A-módulo $M$ tem una série de composição de comprimento finito, então todas as outras séries de composição de $M$ têm comprimento finito e, além disso, todas clas tên o mesmo comprimento.

O 'leorema 1.1 .5 permite definir o comprinento de um $A$-módulo $M$.

Definição 1.1.6: O comprimento de un A-módulo $M$, denotado por $L_{A}(M)$, é 
definido como o comprimento de qualquer série de composição de $M$. Neste caso, diremos (ple $M$ tem comprimento finito.

Afirmar que um A-módulo $M$ ć Noetheriano, é equivalente a dizer que todos os seus submódulos são finitamente gerados, mas isto não significa dizer que cle ten comprimento finito. O próximo resultado esclarece definitivamente esta questão.

Teorema 1.1.7: ([1], pag. 77) Um A-módulo $M$ tem uma série de composição se, c somente se, satisfaz ambas as condições de cadeia.

Por exemplo, o anel $k\left[x_{1}, \ldots, x_{n}\right]$ ć Noctheriano, polo Teorcma 1.1.2, mas não tem comprimento finito, pois não satisfaz a condição de cadeia descendente. Basta, por excmplo, tomar a cadeia descendente

$$
(x) \supset\left(x^{2}\right) \supset \cdots \supset\left(x^{n}\right) \supset \cdots,
$$

que não é estacionária.

Definição 1.1.8: Seja $\mathcal{C}$ uma classe de $A$-módulos. Uma furçãa $\mathcal{L}$ de $\mathcal{C}$ em $\mathbb{Z}$ ć chamada aditiva, se para toda sequência exata da forma

$$
0 \longrightarrow M^{\prime} \longrightarrow M \longrightarrow M^{\prime \prime} \longrightarrow 0
$$

onde $M, M^{\prime}$ e $M^{\prime \prime}$ pertencem a $\mathcal{C}$, tem-se

$$
\mathcal{L}\left(M^{\prime}\right)-\mathcal{L}(M)+\mathcal{L}\left(M^{\prime \prime}\right)=0
$$

Por exemplo, o comprimento $L_{A}(M)$, c uma função aditiva na classe de todos os A-módulos de comprimento finito. Mais precisamente, se

$$
0 \rightarrow M^{\prime}>M->M^{\prime \prime}>0
$$

ć uma sequência exata de $A$-módulos, ambos de comprimento finito, então

$$
L(M)=L\left(M^{\prime}\right)+L\left(M^{\prime \prime}\right)
$$

O mesmo acontece para a dimensão de espaços vetoriais, $\operatorname{dim}_{k}(V)$, onde $V$ a um espaço vetorial de dimensão finita sobre o corpo $k$. 
Um elemento $a \in A$, é chamado um divisor do zero em $A$, se existe um elemento não nulo $y \in A$, tal que $a y=0$. O conjunto dos divisores do zero num anel Noctheriano $A$, e caracterizado como a união de todos os ideais primos minimais do ideal rumlo do A ([1: pag. 5:3).

Un ideal I do anel $A$ é chamado primário, se qualquer divisor do zero no ancl $A / I$ for um elemento nilpotente, ou equivalentemente, se $a, b \in A$ com $a b \in I$ o $a$ não pertence a $I$, entào existe $n \in \mathbb{N}$ (dependendo de $b$ e $I$ ), tal cue $b^{n} \in I$.

Seja $I$ um ideal do anel A. O conjunto

$$
\left\{a \in A \text { tais que } a^{n} \subset l \text {, para algum inteiro } n>0\right\}
$$

cum ideal de $A$, chamado o radical do ideal $I$ có denotado por $\sqrt{I}$. Claramentr $I \subset$ $\sqrt{I}$. No caso particular, em que $I \ldots 0, \sqrt{0}$ é o nilradical de $A$. Outras propriedades que usaremos do radical são as seguintes:

i) Sejam $I$ e $J$ dois ideais de um anel $A$. Então $\sqrt{I J}=\sqrt{I \cap J}-\sqrt{I} \cap \sqrt{J}$.

ii) Se $\mathcal{P}$ ó um ideal primo de $A$, entăo $\sqrt{\mathcal{P}^{t}}=\mathcal{P}$, para todo intciro $n>0$.

Observaçāo 1.1.9: Sejam $I$ a $J$ dois ideais $\mathcal{M}$-primários do ancl local $(A, \mathcal{M})$. Fntão dados $r$ e s inteiros positivos, o ideal $I^{r} J^{s}$ é $\mathcal{M}$-primário, pelo item (ii) acima. $N_{a}$ verdade, esta afirmação vale para um númoro finito de ideais $\mathcal{M}$-primários.

Scja $(A, \mathcal{M})$ um anel local. Tom ideal $I$ de $(A, \mathcal{M})$ o chamado $\mathcal{M}$-primátio se $\sqrt{I}-\mathcal{M}$. Mais geralmente, se $\mathcal{P}$ é um ideal primo de $A$, um ideal $I$ de $A$ échamado $\mathcal{P}$-primário se $\sqrt{I}=\mathcal{P}$. Por exemplo, o radical de um ideal primário é um ideal primo.

() próximo resultado, fornece uma caracterização muito útil des ideais $\mathcal{M}$-primários do $u$ mancl local $(A, \mathcal{M})$.

Teorema 1.1.10: ([12], pag, 23) Scja $I$ um ideal do ancl Noctheriano local $(A, \mathcal{M})$. Então un ideal $I$ de $A$ é $\mathcal{M}$-primário se, esomente se, contém alguma potência de $\mathcal{M}$, isto é, existe um inteiro positivo $r$ (dependendo do $I$ e $\mathcal{M}$ ), tal que $\mathcal{M}^{r} \subseteq I$. 
Vamos definir o comprimento de um ideal $\mathcal{M}$-primário $I$ num anel Noctheriano local $(A, \mathcal{M})$. Para isto, suponha que que $I_{1}, \ldots, I_{s}$ são ideais $\mathcal{M}$-primários tais que

$$
I=I_{1} \subset \cdots \subset I_{s}=\mathcal{M},
$$

onde todas as inclusões são estritas. Descrevemos esta situação, dizendo que $\left[I_{1}, \ldots, I_{s}\right]$ é uma cadeia primária que começa no ideal $I$ e termina no ideal maximal $\mathcal{M}$, ou simplesmente, que é uma cadcia primária do idcal $I$ ao ideal $\mathcal{M}$. Toda cadeia primária de $I$ a $\mathcal{M}$, pode ser refinada numa série de composição ([12], pag. 52) de $I$. Observe que se $I$ é um ideal do anel $(A, \mathcal{M})$ tal que

$$
I \subset J \subset \mathcal{M}
$$

então desde que I é um ideal $\mathcal{M}$-primário, podemos encontrar, pelo Tcorema 1.1 .10 um inteiro positivo $r$, tal que

$$
\mathcal{M}^{r} \subseteq I \subseteq \sqrt{J} \subseteq \mathcal{M}
$$

c aplicando radicais

$$
\mathcal{M}=\sqrt{\mathcal{M}^{r}} \subseteq \sqrt{I} \subseteq J \subseteq \sqrt{\mathcal{M}}=M
$$

Portanto, o ideal $J$ deve ser $\mathcal{M}$-primário. Em outras palavras, isto significa que os ideais $\mathcal{M}$-primários que aparecem entre $I$ o $\mathcal{M}$, são exatamente os idcais $J$ do anel $A$ que aparecem entre os idcais $I \circ \mathcal{M}$. Prova-se ainda, ([12],pag. 54), que existe uma correspondência bijetiva entre as cadcias de ideais do $I$ a $\mathcal{M}$ e as cadeias de ideais entre $\mathcal{M} / I$ e o ideal nulo de $A / I$. Podemos então definir o comprimento de ideais $\mathcal{M}$-primários do seguinte modo.

Definiçāo 1.1.11: Seja $I$ um ideal $\mathcal{M}$-primário de um anel Noctheriano local $(A, \mathcal{M})$. Definimos o comprimento do ideal $I$, denotado por $L_{A}(I)$, por

$$
L_{A}(l)=L_{A}(A / I)
$$

Definição 1.1.12: Seja $I$ um ideal do ancl $A$. So o ideal $I$ pode ser expresso como uma intersecção finita de ideais primários, isto é,

$$
I=I_{1} \cap \cdots \cap I_{n} .
$$


onde cada $I_{i}, i=1, \ldots, n$ é um ideal primário de $A$, então dizemos que a expressão (1.2) ć uma decomposiçáo primária do ideal $I$. Cada ideal $I_{i}, i=1, \ldots, n$ que aparece em (1.2), 6 chamado uma componente primária de $I$. Todo ideal $I$ de $A$ que admite uma decomposiçăo primária é chamado decomponível.

Uma decomposição primária em que todos os $\sqrt{I_{i}}$ são distintos e cada ideal $I_{i}$ não contém a interseção dos demais, é chamada decomposiçâo irredundanle ou decomposiçäro normal.

Toda decomposição primária pode ser refinada até uma decomposição normal. Além disso, todo ideal $I$ de um anel Noetheriano $A$ admite uma docomposição primária.

Definiçāo 1.1.13: Seja $I$ um ideal do ancl $A$. Um ideal primo $\mathcal{P}$ de $A$ é chamado um ideal primo minimal de $I$, se cle contém $I$ o não existe nenhum outro ideal primo de $A$ que esteja contido estritamente entre $\mathcal{P} \cap I$.

Pocte-se caracterizar o conjunto dos elementos nilpotentes num anel Noctheriano A, em função dos ideais primos minimais do ideal nulo da seguinte mameira. o conjunto dos elementos nilpotentes do ancl $A$, ć a intersecção de todos os ideais primos minimais do ideal nulo de $A$ ([1], pag. 53).

A conexão entre o radical de um idoal $I$ num anel $A$ e seus ideais primos minimais está explicada no próximo tcoroma.

Teorema 1.1.14: ([12], pag. 14) Suponha que

$$
I=I_{1} \cap I_{2} \cap \ldots \cap I_{n}
$$

scja uma decomposição primária do ideal $I$, onde cada $I_{i}$ é $\mathcal{P}_{i}$-primário para $1 \leq i \leq n$. Então valem as seguintes afirmações:

i) Qualquer ideal primo $\mathcal{P}$ que contém $I$, necessariamente contém pelo monos um dos ideais $\mathcal{P}_{i}$.

ii) Os ideais primos minimais de $I$. são exatamente os ideais primos $\mathcal{P}_{i}$, que não contenham estritamente qualguer outro $\mathcal{P}_{j}$, para $i \neq j$. 
iii) Temos

$$
\sqrt{I}=\mathcal{P}_{1} \cap \ldots \cap \mathcal{P}_{n}
$$

Além disso, o radical de $I$, ć a intersecção de todos os ideais primos minimais de $I$.

() Teorema 1.1.14, mostra que so I é um ideal decomponívol de $A$, então existe apenas um número finito de ideais primos minimais de $I$ e que eles estão associados com cada decomposiçâo primária de $I$.

Teorema 1.1.15: ([12], pag. 15) Suponha que o ideal $I$ admita uma decomposição primária e, além disso, tenhamos

$$
I=I_{1} \cap \ldots \cap I_{m}=\tilde{I}_{1} \cap \ldots \cap \tilde{I}_{n}
$$

duas decomposiçoos primárias de $I$, onde $I_{i} 6 \mathcal{P}_{i}$ primário para $i-1, \ldots, m$ e $I_{j} 6$ $\tilde{\mathcal{P}}_{j}$-primário, para $j=1, \ldots, n$. Então $m=n$, é possível ordenar as componentes de modo que $\mathcal{P}_{i}=\tilde{\mathcal{P}}_{i}$ jara $1 \leq i \leq m=n$.

Em outras palavras, o Teorema 1.1.15, mostra que se $I$ é um ideal decomponível de $A$, então os ideais primos que que aparecem na decomposição normal de $I$ não dependem de nenhuma decomposiçẫo normal particular, mas apenas do ideal $I$. Fstes ideais primos serão chamados do associados de $I$, on também são ditos pertencer a I. Pelo Teorema 1.1.14, todo ideal primo minimal de $I$, pertence a $I$ neste sentido. Os ideais primos associados, que nâo são minimais, são chamados de imersos. () conjunto dos ideais primos associados de $I$ será denotado por Ass $(I)$.

Por uma cadeia de ideais primos do anel $A$, entendemos uma sequencia estritamente ascendente de ideais primos de $A$

$$
\mathcal{P}_{0} \subset \mathcal{P}_{1} \subset \cdots \subset \mathcal{P}_{k}
$$

e diremos que k é o comprimento da cadeia.

Definição 1.1.16: Definimos a dimensăo de Krull de um ancl $A$, como sendo o supremo dos comprimentos de todas as cadeias de idcais primos em $A$ e a denotaremos 
por $\operatorname{dim} A$. Para um ideal primo $\mathcal{P}$ do anel $A$, o supremo dos comprimentos, tomados sobre todas as cadeias cstritamente decrescentes de ideais primos

$$
\mathcal{P}_{0} \subset \mathcal{P}_{1} \subset \cdots \subset \mathcal{P}_{k}=\mathcal{P}
$$

e chamado a altura de $\mathcal{P}$ e denotado por htP. Se $I$ ć um ideal de $A$, definimos a alturat de $I$, denotada por $h t I$ como sendo o ínfino das alturas dos ideais primos contendo I.

Observação 1.1.17: Convém observar que, dim $A$ é um número inteiro não-negativo $011+\infty$.

Exemplo 1.1.18: 1) Todo corpo tem dimensão 0

$O$ anel dos inteiros $\mathbb{Z}$ tem dimensão 1. Mais geralmente, todo domínio principal que não é corpo, tem dimensão 1, pois num domínio principal, todo ideal primo é maximal.

2) ([9], pag. 35) Soja $k$ um corpo. Então o anel dos polinómios $k\left[x_{1}, \ldots, x_{n}\right]$ e o ancl das sćries de potências $k\left\{x_{1}, \ldots x_{n}\right\}$ têm dimensảo $n$.

\subsection{Módulos Cohen-Macaulay}

Seja $A$ un ancl Noetheriano local com ideal maximal $\mathcal{M}$. Soja $M$ um $A$-módulo finitamente gerado. O anulador de $M$, c o conjunto

$$
A n n(M)=\{a \in A: a m=0 \text { para todo } m \in M\} .
$$

Mostra-se que $A n n(M)$ é um ideal de $A$

A dimensão de $M$, denotada por $\operatorname{dim} M$, é definida como a dimensão de Krull do anel $A / \operatorname{Ann}(M)$, isto é,

$$
\operatorname{dim} M=\operatorname{dim}(\Lambda / \operatorname{Am}(M))
$$

Note que quando $A n n(M)=\{0\}$, tem-se $\operatorname{dim} M=\operatorname{dim} A$

Seja $(A, \mathcal{M})$ um anel Noetheriano local $r$-dimensional, isto é, $\operatorname{dim} A=r$. So os elementos $a_{1}, \ldots, a_{r} \in \mathcal{M}$, geram um ideal $\mathcal{M}$-primário, dizemos cue $\left\{a_{1}, \ldots, a_{r}\right\}$ o um sistema de parâmetros de $A$ 
Seja $M u m A$-módulo finitamente gerado e $\operatorname{dim} M=s$. Suponha que os elementos $a_{1}, \ldots, a_{s} \in \mathcal{M}$ tenham a seguint.c propricdade: o $A$-módulo

$$
M /\left(a_{1} M+\ldots+a_{s} M\right)
$$

tem comprimento finito. Neste caso, dizemos que $a_{1}, \ldots, a_{s}$ é um sistcma de parâmetros de $M$. Observe que estamos exigindo que o número de elementos num sistema de parâmetros de $M$ seja exatamente igual a dimensão de $M$. Um ideal que é gerado por um sistema de parâmetros ó chamado um ideal parâmetro de $A$.

Lm elemento $a \in A$ ć dito ser $M$-regular se $a m=0$ para todo $m \in M, m \neq 0$ implicue $a=0$.

Definição 1.2.1: Scja $(A, \mathcal{M})$ um ancl Noetheriano local. Uma sequência $a_{1}, \ldots, a_{n} \in$ $\mathcal{M}$ de elementos de $A$ é chamada uma $M$-sequência se as seguintes condições forem satisfeitas:

i) $a_{1}$ é $M$-regular, $a_{2}$ é $\left(M / a_{1} M\right)$-regular, $\ldots, a_{n}$ é $\left(M /\left(a_{1}, \ldots, a_{n-1}\right) M\right)$-regular;

ii) $M /\left(a_{1}, \ldots, a_{n}\right) M \neq 0$.

Suponha que $M \neq 0$. Qualquer $M$-sequencia pode ser extendida até uma $M$ sequência maximal. De fato, pois caso contrário, existiria uma sequência infinita $\left(a_{1}, a_{2}, \ldots\right)$ tendo a propriedade $(i)$ da Definição 1.2 .1 e uma scquência ascendente de ideais

$$
\left(a_{1}\right) \subset\left(a_{1}, a_{2}\right) \subset \ldots
$$

o que contradiria o fato de $A$ scr um ancl Noctheriano. Prova-se ainda, que todas as $M$-seqüencias maximais tôm o mesmo número de elementos e, além disso, toda $M$-sequência pode ser extendida a un sistema de parâmetros ([21], pag. 61).

Definição 1.2.2: $O$ número de elementos numa $M$-sequência maximal de $M$ é chamada a profundidude de $M$, cé denotada por deptha $M$. Um módulo $M$ é chamado módulo Cohen-Macaulay se

$$
\operatorname{dim} M=\operatorname{depth} M
$$


Lm anel A é chamado anel Cohen-Macaulay, se ele é um módulo Cohen-Macaulay quando visto como un módulo sobre si mesmo.

O próximo resultado, fornece uma caracterização muito útil de módulos CohenMacaulay .

Teorema 1.2.3: ([21], pag. 65) Se $M$ ć um módulo Cohen-Macaulay, todo sist.ema de parämetros de $M$ é uma $M$-sequencia. Reciprocamente, se um sistema de paràmetros de $M$ é uma $M$-sequência, entào $M$ é um módulo Cohen-Macaulay.

Finalmente, cncerramos esta seção com um exemplo de um módulo Cohen-Macaulay

Exemplo 1.2.4: Seja $k$ um corpo e considere os anéis $A=k\left[x_{1}, \ldots, x_{n}\right]$ e $B=$ $\left\{x_{1}, \ldots, x_{n}\right\}$. Entáo $A$ e $B$ são módulos de Cohen-Macaulay. Neste caso, $x_{1}, \ldots, x_{n}$, desempenham o pajel de uma $M$-sequência regular em $A$.

\subsection{Anéis e Módulos Graduados}

Definiçāo 1.3.1: Seja $T$ um conjunto nào-vazio. Um monóide de graduaçäo é um par $(T,+)$, onde

$$
+: T \times T \longrightarrow T
$$

é uma operação binária em $T$, satisfazendo os seguintes axiomas:

i) Para quaisquer $a, b$ pertencentes a $T$, vale a comutatividade, isto $c$,

$$
a+b=b+a
$$

ii) Para quaisquer $a, b, c$ pertencentes a $T$, vale a associatividade, ou seja

$$
a+(b+c)=(a+b)+c
$$

iii) Existe um elemento neutro cm $T$, denotado por 0 , tal que

$$
a+0=a=0+a,
$$

para qualquer $a \in T$;

iv) Se $a+b=a+c$, onde $a, b$, r cstão $\mathrm{cm} T$, então $b=c$. 
Observaçāo 1.3.2: 0 elemento 0 do item (iii) é único.

Exemplo 1.3.3: 1) Qualquer grupo abeliano escrito aditivamente é um monóide de gradıação;

2) O conjunto dos números naturais, com a adição usual, é um monóide de graduação;

3) O conjunto das n-uplas de números naturais, com a adição definida coordenada a coordenada, também ć um monóide de graduaçào.

Sejam $T$ um monóide de graduação e $A$ um anel. Os elementos de $A$ formam um grupo abcliano com relação à adição. Não faremos distinção entre o anel $A$ e o grupo abeliano $A$.

Definição 1.3.4: Uma $T$-graduação em $A$ c uma família $\{A \gamma\}_{\gamma \in T}$ de subgrupos do grupo $A$ tal que:

i) $A=\oplus_{\gamma \subset T} A_{\gamma}$;

ii) $A_{\gamma} A_{\beta} \subseteq A_{\gamma+\beta}$.

Neste caso dizemos que $A$ é um anel $T$-graduado.

Observação 1.3.5: Sejam $A$ um ancl e $T$ um monóide de graduaşão. Defina $A_{0}=A$ e $A_{\gamma}=0$ para todo $\gamma \in T-\{0\}$. Então $\left\{A_{\gamma}\right\}_{\gamma \in T}$ ć uma $T$-graduação em $A$. Portanto, dados um monóide de graduação $T$ e um anel $A$, sempre é possível definir uma $T$ graduação em $A$. Esta graduação é chamada de $T$-graduação lrivial, on de graduação natural.

Suponhamos que $A$ seja um ancl $T$-graduado. Pelo item (i) da Definição 1.3.4, cada elemento $r$ de $A$, admite uma única representação da forma

$$
r=\sum_{\gamma \in T} r_{\gamma} \text {, onde } r_{\gamma} \in A_{\gamma}
$$

c. alúm disso, esta soma contém somente um número finito de termos não nulos.

Os elementos de $A_{\gamma}$, são ditos ser homogêneos. ou formas, de grau $\gamma$ e $r_{\gamma}$ é chamado a componente homogênea de $r$ de gran $\gamma$. 
Observação 1.3.6: 1) Por convenção, o clemento zero é homogêneo de grau $\gamma$, para todo $y \subset T$;

2) O elemento identidade de $A$ é homogènco de gran zero:

3) $A_{0}$ ć um subanel de $A$ c todos os seus elementos säo homogeneos de grau zero.

Scjam $A=\oplus_{\gamma \in T} A_{\gamma}$ um anel $T$-graduado e $M$ urn $A$-módulo. Os elementos de $M$ formam um grupo abeliano com relação à adição. Por abuso de notação, continuaremos a denotar este grupo por $M$.

Definição 1.3.7: Una $T$-graduação em $M$, é una família $\left\{M_{\beta}\right\}_{\beta \in T}$ de subgrupos do grupo abeliano $M$ que satisfaz:

i) $M-\oplus_{B \in T} M_{\beta}$ :

ii) $A_{\gamma} M_{\beta} \subset M_{\gamma+\beta}$, para quaisquer $\gamma, \beta \in T$.

Neste caso, dizcmos que $M$ ó um módulo T-graduado sobre o ancl $T$-graduado $A=$ $\mathbb{1}_{\gamma \subset T} A_{\gamma}$

Observação 1.3.8: Todo $A$-módulo $M$ sempre admite uma graduação. De fato, so A é um anel com a graduação trivial, basta definirmos $M_{0}=M \circ M_{\beta}=0$, para cada $\beta \in T-\{0\}$. Da mesma forma, dizemos que esta $T$-graduação ć a graduação trivial ou a graduação natural de $M$.

Seja $M-\oplus_{\beta \in T} M_{\beta}$ um módulo $T$-graduado sobre o anel $A=\oplus_{\gamma \in T} A_{\gamma}$. Então cada elemento $m \in M$ admite uma única representação da forma

$$
m=\sum_{\beta \in T} m_{\beta} \text { onde } m_{\beta} \in M_{\beta}
$$

e a soma contém somente um número finito de ternos năo-nulos.

Observação 1.3.9: Segue do itemn (ii) da Definição 1.3.7, que $M_{\beta}$ é um $A_{0}$-módulo para cada $\beta \in T$. Assim, $M=\Phi_{3 c T} M_{3}$, ́́ uma decomposição em soma direta de $M$ como $A_{0}$-módulo.

Proposição 1.3.10: (13], pag. 115) Seja $K$ um submódulo do $A$-módulo $T$ graduado, $M=\oplus_{\beta} M_{\beta}$. Então as seguintes afirmaçôes são equivalentes: 
i) $K=\oplus_{\beta \in T}\left(M_{\beta} \cap K\right)$

ii) Se $m \in K$, então todas as componentes homogêneas de $m$ pertencem a $K$;

iii) $K$ pode scr gerado, como $A$-módulo, por elementos homogêneos.

Definição 1.3.11: Um submódulo $K$ de um $T$-módulo graduado $M$ que satisfaz qualquer uma das propriedades equivalentes da Proposição 1.3.10, é chamado de submódulo homogêneo de $M$. Os submódulos graduados de $A$, são chamados os ideais graduados de $A$.

Vamos citar várias propriedades dos ideais graduados. Suas demonstrações podem ser encontradas em ([34], pag. $152-153$ ).

Teorema 1.3.12: Sejam $I$ e $J$ dois idcais homogêneos de um anel graduado $A$. Entäo valem as seguintes propriedades.

1) $I+J, I J, I \cap J, \sqrt{I}$, são ideais homogêneos de $A$.

2) Se $I$ admite una decomposição primária da forma $I=\cap_{j=1}^{n} I_{j}$, então cle também admite uma decomposição primária $I=\cap_{j=1}^{n} I_{j}^{\star}$, onde $I_{j}^{\star}$ são ideais primários homogênecos, para $j=1, \ldots, n$.

Sejam $M=\oplus_{\beta \in T} M_{\beta}$ um módulo $T$-graduado sobre o anel $A$ e $K$ um submódulo homogêneo de $M$. So definimos

$$
K_{\beta}=M_{\beta} \cap K
$$

então a família $\left\{K_{\beta}\right\}_{\beta \in T}$ ć uma $T$-graduação cm $K$. Fista graduação, ć chamada a $T$-graduaçâo induzida em $K$ pela graduação de $M$.

Ainda, assumindo que $K$ seja um submódulo homogêneo de $M$, considere

$$
\phi: M \longrightarrow M / K
$$

onde $\phi$ é a projeção canônica. Agora como $M \mathrm{e} M / K$ podem ser considerados como $A_{0}$-módulos, e portanto, $\phi$ pode ser considerada como um $A_{0}$-homomorfismo de $A_{0^{-}}$ módulos. Definimos

$$
\phi\left(M_{\beta}\right)=(M / K)_{\beta}
$$


para todo $\beta \in T$. Entrào $(M / K)_{3}$ é um $A_{0}$-submódulo do $M / K$ e portanto, um subgrupo do grupo aditivo $M / K$. Além disso, $\left\{(M / K)_{\beta}\right\}$ é uma $T$-graduação em $M / K$, chamada de $T$-graduaçâ quociente.

Exemplo 1.3.13: Scjam $T=\mathbb{N}, k=\mathbb{R}$ on $\mathbb{C}$ e $A \because k \mid x_{1}, \ldots x_{n} \vdots$ Então com a notação usual, família $\left\{(k[X])_{r}\right\}_{r \in \mathbb{N}}$, onde $(k[X])_{r}$ é o subgrupo do $k[X]$ formado pelos polinominos homogêneos de grau $r \in \mathbb{N}$, é uma $\mathbb{N}$-graduação de $A=k[X]$.

Seja $I$ um ideal homogèneo de $k[X]$. Entäo a familia $\left\{(k:[X] / I)_{r}\right\}_{r c s}$, onde $(k:[X] / I)_{r}=\left(k:[X]_{r}+I\right) / I$ é uma $\mathbb{N}$-graduação para o ancl $k:[X] / I$, isto é,

$$
k[X] / I-\mathrm{O}_{r} \mathrm{r}(k[X] / I)_{r}
$$

Definição 1.3.14: Sejam $M=\Theta_{\beta \in T} M_{\beta}$ e $N=\mathbb{D}_{\beta \in T} N_{\beta}$ dois $A$-módulos $T$-graduados. Suponha quo

$$
\int: M \longrightarrow N
$$

seja um homomorfismo de $A$-módulos. So existe $\gamma \in T$ tal que

$$
f\left(M_{3}\right) \subseteq N_{\beta+\gamma}
$$

para todo $\beta \in T$, dizemos que $f$ é um homomorfismo de grau $\gamma$ entre $A$-módulos graduados. So $\gamma=0$, dizemos que $f$ preserva grau.

Sejan I um ideal do anel $A$ o $M$ um $A$-módulo. Considere os seguintos grupos abelianos

$$
G_{I}(A)=\oplus_{n \cdot 0}^{\infty} I^{n} / I^{n+1}
$$

e

$$
G_{I}(M)=\oplus_{n=0}^{\infty} I^{n} M / I^{n+1} M
$$

É possível ([34], pag. 248) introduzir uma estrutura de anel em $C_{I}(A)$ que o torna unn anel graduado onde os clementos de $I^{n} / I^{n+1}$ são os clementos homogeneos de grau $n$. Como $G_{l}(A)$ é un anel graduado pode-se munir $G_{l}(M)$ com uma estrutura de $G_{I}(A)$-móxlulo graduado. Dizemos que $G_{1}(A)$ ó o anel graduado associado de $A$ com relação a $I$ e que $G_{I}(M)$ é o módulo graduado associado a $M$ com relação ao ideal $I$. 


\subsection{Singularidades}

Sejam $z$ um ponto arbitrário de $\mathbb{C}^{n}$ e $\mathcal{V}$ uma vizinhança aberta de $z$ em $\mathbb{C}^{n}$. Considere o seguinte conjunto

$$
S-\left\{h: \mathcal{V} \rightarrow \mathbb{C} \text { onde } \mathcal{V} \subset \mathbb{C}^{n} \text { é uma vizinhanga aberta de } z\right\},
$$

das aplicações contínuas definidas numa vizinhança aberta do ponto $z \in \mathbb{C}^{n} \mathrm{~cm} \mathbb{C}$.

Definição 1.4.1: Dizemos que duas funções contínuas $f: \mathcal{V} \longrightarrow \mathbb{C}$ e $g: \mathcal{U} \longrightarrow \mathbb{C}$, são equivalentes no ponto z se:

i) existe uma vizinhança $\mathcal{W}$ de z tal que $\mathcal{W} \subset \mathcal{V} \cap \mathcal{U}$;

ii) $f_{I_{w}}(w)=g_{w}(w)$, para tollo $w \in \mathcal{W}$.

Denotamos por $f \sim_{z} g$, o fato de $f$ ser equivalente a $g$ no ponto z. Convém observar, que $\sim_{z}$ é uma relaçào de equivalência no conjunto de todas as funçóes contínuas de $\mathbb{C}^{n}$ em $\mathbb{C}$. Cada classe de equivaloncia ó chamada de um germe de aplicaçoes contínuas. Fim particular, o conjunto de todas as funções contínuas que säo equivalentes a $f$ no ponto $z \in \mathbb{C}^{n}$, ó chamado o germe da $f$, com relabăo ao ponto $z$, denotado por $[f]$.

Observação 1.4.2: 1) O item $i$ ), nos diz que o germe de uma função $f$ num ponto $z$ de $\mathbb{C}^{n}$, depende do comportamento da funçăo em toda uma vizinhança aberta $\mathcal{W}$ de z, nào somente do valor da fumçăo no ponto z. Por exemplo, os polinomios z e $z^{2}$ tem o mesmo valor na origem do pldino complexo, mas determinam germes bem distintos na origem. Para ver isto, basta considerar cstes dois polinômios restritos, por exemplo, ao cixo real, pois neste cixo, $z \neq z^{2}$, para toda vizinhança abertid $\mathcal{U}-\{0\}$.

2) O conjunto de germes das funções contínuas pode ser munido de urma estrutura de anel como segue. l'ara quaisquer dois germes $[f] \mathrm{e}[g] \mathrm{em} z$ selecione representantes $f_{\mathcal{U}}$ e $g \mathcal{V}$, respectivamente. Se $\mathcal{W}$ é uma vizinhança abcrta de $z$ tal que $\mathcal{W} \subseteq \mathcal{U} \cap \mathcal{V}$ o germe da função $f_{U}|W+g \mathcal{W}| W$ ó definido como a soma $\left.[f]+\mid g\right]$ a o germe da função $\left(f_{Z} \mid \mathcal{W}\right)\left(g_{\mathcal{V}} \mid \mathcal{W}\right)$ é definido como sendo o produto $[f][g]$. Verifica-se que estas operaçoes säo bem definidas, no sentido de serem independentes da eseolha dos representantes. 
Se considerarmos apenas as funções analíticas complexas de $\mathbb{C}^{n}$ em $\mathbb{C}$, o anel resultante scrá chamalo o anel dos germes analíticos, e o germe de mana função analítica complexa é chamado de germe analítico. Os gemes analíticos de $\mathbb{C}^{n}$ em $\mathbb{C}$, no ponto $z$, serão denotados por $\mathcal{O}_{n . z}$. So $z=0$, então $\mathcal{O}_{n, 0}$ será simplesmente demotado por $\mathcal{O}_{n}$.

As translações de $\mathbb{C}^{n}$ em $\mathbb{C}^{n}$ estabelecem um isomorfismo entre os anéis $\mathcal{O}_{n, z}$ e $\mathcal{O}_{n}$. Mais precisamente, a aplicação

$$
\mathcal{O}_{n, a} \longrightarrow \mathcal{O}_{n}
$$

definida por $f \longmapsto g$, onde $g(x)=f(x-a)$, ó $1 \mathrm{~mm}$ isomorfismo. Estes isomorfismos preservam todas as propriedades analíticas de interesse e, portanto, basta estudar apenas as propriedades analíticas do anel $\mathcal{O}_{n}$.

Pode-se provar que o ancl $\mathcal{O}_{n}$ goza das seguintes propriedades algébricas:

i) $\left([6]\right.$, pag. 3) $\mathcal{O}_{n}$ ć isomorfo ao ancl $\mathbb{C}\left\{x_{1}, \ldots, x_{n}\right\}$ das séries de potências convergentes em $n$ indeterminadas sobro $\mathbb{C}$, que convergem na origem;

ii) ([6], pag. 7) $\mathcal{O}_{n}$ é um ancl Noctheriano. 


\section{Capítulo 2}

\section{Multiplicidades}

\subsection{Introdução}

As idéias modernas da teoria da multiplicidade emergiram da aplicacão da teoria das furcecoes de Hilbert ans anósis locais. Neste capútulo, desenvolveremos a teoria da multiplicidade algébrica, também chamada de multiplicidade de Samuel. Uma definição indutiva será dada para o símbolo da multiplicidade geral para $s$ elementos $\gamma_{1}, \ldots, \gamma_{s}$ de um anel $A$, relativarnente a um $A$-módulo $M$ de modo que o comprimento

$$
L_{A}\left(M /\left(a_{1}, \ldots, a_{r}\right) M\right)
$$

seja finito. O conceito de multiplicidade surge a partir da definição de comprimento. Embora a definição de comprimento não exige que o anel $A$ scja comutativo, voltamos a enfatizar (1ıe por todo o nosso trabalho, a palavra anel, deverá ser sempre entendida (como anel Noetheriano commtativo con unidade.

Os resultados deste capítulo, encontram-se basicamente nas seguintes referencias: [13] e [32] c a definição de multiplicidade que daremos na segunda segão, difere ligeiramente da definição devida a [32]. 'Tratamentos diferentes do adpi apresentado, podem ser encontrados $\mathrm{em}[9],[10]$.

Segundo alguns autores, o artigo [20], marca o início do tratamento moderno da teoria da multiplicidade, como é contuecida atualmente. 


\subsection{Sistemas de Multiplicidades}

Nesta seção, definiremos sistemas de multiplicidades e estudaremos algumas de suas principais propriedades, que utilizaremos no desenvolvimento deste capítulo. Os resultados aqui apresentados fazem parte do livro [13].

Sejam $a_{1}, \ldots, a_{r}$ elementos arbitrários do anel $A$. Escreveremos $I=\left(a_{1}, \ldots, a_{r}\right)$, para indicar o ideal de $A$, gerado por estes elementos. Por outro lado, escreveremos, $I M$, para denotar o submódulo $a_{1} M+\ldots+a_{r} M$ de $M$. Outra notaçãa, que eventualmente podemos usar á a seguinte: $\left(a_{3}, \ldots, a_{s}\right) M$.

Definição 2.2.1: Sejam $M$ um A-módulo e $\gamma_{1}, \ldots, \gamma_{s}$ elementos do anel $A$. Dizemos que $\gamma_{1}, \ldots, \gamma_{s}$ é um sistema de multiplicidade $\mathrm{em} M$, se o A-módulo

$$
M /\left(\gamma_{1} M+\ldots+\gamma_{r} M\right)
$$

tem comprimento finito. Quando $s=0$, entende-se que $M$ tem comprimento finito.

Sejam $A$ um anel Noetheriano local, $M$ um $A$-módulo finitamente gerado e $x_{1}, \ldots, x_{r}$, um sistema de parâmetros para $M$. Então, pela Definição 2.2.1, todo sistema de parâmetros para $M$ também ć ım sistema de multiplicidade para $M$.

Exemplo 2.2.2: Sejam $k$ um corpo e $x_{1}, \ldots, x_{s}$ um conjunto finito de indeterminadas sobre $k$. Então o conjunto $\left\{x_{1}, \ldots, x_{s}\right\}$ é um sistema de multiplicidade, tanto no anel dos polinômios $A=k\left[x_{1}, \ldots, x_{s}\right]$, quanto no anel das séries de potências formais $A^{\prime}=k\left\{x_{1}, \ldots, x_{s}\right\}$. De fato, desde que

$$
A /\left(x_{1}, \ldots, x_{s}\right) A \simeq k: A^{\prime} /\left(x_{1}, \ldots, x_{s}\right) A^{\prime} \cong k
$$

segue da Definição 2.2.1, que $x_{1}, \ldots, x_{s}$ é um sistema de multiplicidade tanto em $\left.k i x_{1}, \ldots, x_{s}\right]$ quanto em $k\left\{x_{1}, \ldots, x_{s}\right\}$.

As seguintes propriedades sobre sistemas de multiplicidades serão úteis no estudo do símbolo da multiplicidade, que estudaremos na próxima seção. Para não nos estendermos muito, iremos apenas enunciá-las, omilindo suas demonstrações. 
Na proposição abaixo, não é nccessário que os comprimentos sejam finitos.

Proposiçāo 2.2.3: ([13], pag. 296$)$ Sejam $M$ um $A$-módulo e $\gamma_{1}, \ldots, \gamma_{s}$ elementos do anel A. Então temos que

$$
L_{A}\left(M /\left(\gamma_{1}^{n_{1}} M+\ldots+\gamma_{s}^{n_{s}} M\right)\right) \leq n_{1} \cdots n_{s} L_{A}\left(M /\left(\gamma_{1} M+\ldots+\gamma_{s} M\right)\right)
$$

para quaisquer inteiros positivos $n_{1}, \ldots, n_{s}$.

A partir desta proposiçäo, segue facilmente que se $\gamma_{1}, \ldots, \gamma_{s}(s \geq 0)$ c um sistema de nultiplicidade em $M$, entico $\gamma_{1}^{n_{1}}, \ldots, \gamma_{s}^{n_{s}}$ também é um sistema de multiplicidade em $M$, para quaisquer inteiros positivos $n_{1}, \ldots, n_{s}$.

A proposição abaixo, apesar do seu caracter elementar, desempenhará um papel importanie en várias demonstracōos.

Proposição 2.2.4: ([13], pag. 297) Sejam $M u$ um $A$-módulo e $\gamma_{1}, \ldots, \gamma_{s}$ um sistema de multiplicidade $\mathrm{cm} M$. Então temos as seguintes propriedades:

i) Os elementos $\gamma_{1}, \ldots, \gamma_{s}$, permanecen um sistema de multiplicidade en $M$ mesmo se alterarmos a sua ordem. Além disso, eles continuarão a formar um sistema de multiplicidade cm $M$ se excluimmos qualquer $\gamma_{i}$. para o qual tenhamos $\gamma_{i} M=0$.

ii) Para quaisquer inteiros positivos $n_{1}, \ldots, n_{s}$ os elementos $\gamma_{1}^{n_{1}}, \ldots, \gamma_{s}^{n_{s}}$ também formam um sistema de muliplicidade em $M$.

iii) So $M^{\prime}$ é $u m$ módulo quociente de $M$, então $\gamma_{1}, \ldots, \gamma_{s}$ também é um sistema de multiplicidade em $M^{\prime}$.

iv) Seja $\gamma_{1}, \ldots, \gamma_{s}$ um sistema de multiplicidade em $M$ e $J$ um ideal do anel $A$ tal que $J M=0$. Scja $\bar{A}-A / J$. Denote por $\gamma_{i}$ a imagem de $\gamma_{i}$ em $\bar{A}$. Fintão $\bar{\gamma}_{1}, \ldots, \bar{\gamma}_{s}$ é un sistema de multiplicidade em $M$, quando $M$ for considerado como um $A$-módulo.

A próxima proposição, nos fornece uma condiçăo necessária c suficiente, para que dados um A-módulo $M$ e um conjunto de elementos de $A$, decidir so estes são ou não um sistema de multiplicidade $\operatorname{con} M$. 
Proposiçāo 2.2.5: ([13], pag. 296) Sejam

$$
0 \longrightarrow M^{\prime} \longrightarrow M \longrightarrow M^{\prime \prime} \longrightarrow 0
$$

uma sequência exata de $A$-módulos Noetherianos $c \gamma_{1}, \ldots, \gamma_{s}$ elementos do ancl $A$. Então $\gamma_{1}, \ldots, \gamma_{s}$ é um sistema de multiplicidade em $M$ se, e somente se, ó um sistema de multiplicidade nos $A$-módulos $M^{\prime}$ e $M^{\prime \prime}$.

Sejam $K$ um submódulo do $A$-módulo $M$ e $U$ um subconjunto qualquer do anel A. Então o conjunto

$$
\left(K:_{M} U\right)-\{x \in M \mid \gamma x \in K \text { para todo } \gamma \in U\}
$$

é um submódulo de $M$.

Proposição 2.2.6: ([13], pag. 291-292) Scjam $\gamma \circ \gamma^{\prime}$ elementos do anel $A$ e $K \subset L$ submódulos do $A$-módulo $M$. Entrão as seguintes propriedades são verdadeiras:

i) $\left(\left(K:_{M} \gamma\right):_{M} \gamma^{\prime}\right)=\left(K:_{M} \gamma \gamma^{\prime}\right)$;

ii) $\gamma M \cap K=\gamma(K: M \gamma)$;

iii) $M /(K+\gamma M) \cong(M / K) / \gamma(M / K)$

iv) $\left(L:_{M} \gamma\right) / K=\left((L / K):_{M / K} \gamma\right)$

v) $\left(K:_{M} \gamma\right) / K=(0: M / K \gamma)$.

Para definirmos o símbolo da multiplicidade, precisaremos de um resultado bem conhecido em Álgebra Comutativa, mas por conveniència vamos apenas enunciá-lo.

Proposição 2.2.7: ([13], pag. 175) Seja

$$
0 \longrightarrow M^{\prime} \longrightarrow M \longrightarrow M^{\prime \prime} \longrightarrow 0
$$

uma sequencia exata de $A$-módulos. Então $M$ satisfaz a condiçăo maximal para seus submódulos se, e somente se, $M^{\prime}$ e $M^{\prime \prime}$ também satisfazem a condição maximal para seus submódulos. 


\subsection{O Símbolo da Multiplicidade}

Sejam $M$ um A-módulo Noetheriano e $\gamma_{1}, \ldots \gamma_{s}$ clementos do arel $A$ que formam 1 m sistema de multiplicidade em $M$. Vamos definir a multiplicidarle de $\gamma_{1}, \ldots \gamma_{s} \mathrm{em}$ $M$, isto ć, a multiplicidade do ideal $I$, gerado por este sistema de multiplicidade, o qual iremos denotar pelo símbolo

$$
e_{A}\left(\gamma_{1}, \ldots, \gamma_{s} \mid M\right)
$$

Algumas vezes, iremos denotar o símbolo $e_{A}\left(\gamma_{1}, \ldots, \gamma_{s} \mid M\right)$, simplesmente por $e_{A}(I ; M)$, para simplificar a notaçáo, ou ainda, por razòcs tipográficas. Se $M=A$, entào escreveremos $e_{A}(I)$ ou $e(I)$. O simbolo $e_{A}\left(\gamma_{1}, \ldots, \gamma_{s} \mid M\right)$, wai ser um número inteiro, não-negativo e, para defini-lo vamos usar indução sobre s, o número de clementos do sistema de multiplicidade.

Sejam $M$ um $A$-módulo, $I$ o ideal do anel $A$, gerado pelo sistema do multiplicidade $\gamma_{1}, \ldots, \gamma_{s}$, isto $6, I-\left(\gamma_{1}, \ldots, \gamma_{s}\right)$ e $L_{A}($.$) a funçăo comprimento, definida na classe$ de todos os $A$-módulos de comprimento finito.

Se $s=0$, então o conjunto vazio á, por convenção, um sistema de multiplicidade am $M$ e, portanto, $L_{A}(M)$ é finito. Podemos então definir $e_{A}($.$) pela igualdade$

$$
e_{A}(! M)=L_{R}(M)
$$

ou seja, quando $s=0$, o símbolo da multiplicidade está bem definido.

Suponha agora, que $s \geq 1$ e que o símbolo da multiplicidade já foi definido para A-módulos Noetherianos e sistemas de multiplicidades em $M$ com no máximo s - 1 elementos.

Seja $\gamma_{1}, \ldots, \gamma_{s}$ um sistema de multiplicidade em $M$, consistindo de exatamentes elementos distintos. Considere as seguintes seqüencias exatas de $A$-módulos Noetherianos.

$$
\begin{gathered}
0 \longrightarrow \gamma_{1} M \rightarrow M \longrightarrow M / \gamma_{1} M \longrightarrow 0 \\
0 \longrightarrow\left(0:_{M} \gamma_{1}\right) \longrightarrow M \longrightarrow M /\left(0: M \gamma_{1}\right) \cdots \rightarrow 0
\end{gathered}
$$


Pcla Proposição 2.2.7, $M / \gamma_{1} M$ e $\left(0 i_{M} \gamma_{1}\right)$ são $A$-módulos Noetherianos. Então pela Proposição 2.2.5, o conjunto $\gamma_{1}, \ldots, \gamma_{s}$ é um sistema de multiplicidade tanto em $M / \gamma_{1} M$ quanto em $\left(0:_{M} \gamma_{1}\right)$. Mas $\gamma_{1}$ anula os $A$-módulos $M / \gamma_{1} M$ e $\left(0:_{M} \gamma_{1}\right)$. Daí, tomando $\bar{M}=M / \gamma_{1} M$ e $\bar{N}=\left(0:_{M} \gamma_{1}\right)$ temos as seguintes igualdades

$$
\begin{gathered}
\bar{M} /\left\{\gamma_{1} \bar{M}+\ldots+\gamma_{s} \bar{M}\right\}=\bar{M} /\left\{\gamma_{2} \bar{M}+\ldots+\gamma_{s} \bar{M}\right\} \\
N /\left(\gamma_{1} N+\ldots+\gamma_{s} N\right)=N /\left(\gamma_{2} \bar{N}+\ldots+\gamma_{s} \bar{N}\right) .
\end{gathered}
$$

Como

$$
L_{A}\left(\bar{M} /\left\{\gamma_{2} \bar{M}+\ldots+\gamma_{s} \bar{M}\right\}\right)=L_{A}\left(\bar{M} /\left\{\gamma_{1} \bar{M}+\ldots+\gamma_{s} \bar{M}\right\}\right)<\infty
$$

$$
L_{A}\left(\bar{N} /\left(\gamma_{2} \bar{N}+\ldots+\gamma_{s} \bar{N}\right)\right)-L_{A}\left(\bar{N} /\left(\gamma_{1} \bar{N}+\ldots+\gamma_{s} \bar{N}\right)\right)<\infty
$$

segne pela Definição 2.2.1, que o conjunto $\gamma_{2}, \ldots, \gamma_{s}$, é um sistema de multiplicidade tanto em $M / \gamma_{1} M$ (juanto em $\left(0:_{M} \gamma_{1}\right)$. Então, pela hipótese de indução, os símbolos $e_{A}\left(\gamma_{2}, \ldots, \gamma_{s} \mid M / \gamma_{1} M\right) e e_{A}\left(\gamma_{2}, \ldots, \gamma_{s} \mid\left(0:_{M} \gamma_{1}\right)\right)$ estão bem definidos.

Definição 2.3.1: Sejam $M$ um $A$-módulo Noetheriano e $\gamma_{1}, \ldots, \gamma_{s}$ elementos do anel $A$ tais que $\gamma_{1}, \ldots, \gamma_{s}$ é um sistema de multiplicidade em $M$. Definimos a multiplicidade do ideal $I$, gerado por $\gamma_{1}, \ldots, \gamma_{s}$ en $M$. que denotaremos por $e(I ; M)=$ $\epsilon_{1}\left(\gamma_{1}, \ldots, \gamma_{s} \mid M\right)$, pela equação

$$
e_{A}\left(\gamma_{1}, \ldots, \gamma_{s} \mid M\right)=e_{A}\left(\gamma_{2}, \ldots, \gamma_{s} \mid M / \gamma_{1} M\right)-e_{A}\left(\gamma_{2}, \ldots, \gamma_{s} \mid\left(0:_{M} \gamma_{1}\right)\right)
$$

Observação 2.3.2: 1) Apesar da Definição 2.3.1, não parecer muito natural à primeira vista, ela é motivada pelo teorema 3.3 de [2].

2) O cálculo da multiplicidade de um ideal $I$ gerado por um sistema de multiplicidade $\gamma_{1}, \ldots, \gamma_{s}$ é, en geral, muito complicado. Para se ter uma idéia disto, note que para calcularmos $\epsilon_{A}\left(\gamma_{1}, \ldots, \gamma_{s} \mid M\right)$ devcmos saber inicialmente, os valores de $e_{A}\left(\gamma_{2}, \ldots, \gamma_{s} \mid M / \gamma_{1} M\right) \odot e_{A}\left(\gamma_{2}, \ldots, \gamma_{s} \mid\left(0:_{M} \gamma_{1}\right)\right)$. Agora pela Definição 2.3.1, temos

$$
\begin{aligned}
\epsilon_{A}\left(\gamma_{2}, \ldots, \gamma_{s} \mid M / \gamma_{1} M\right)= & \epsilon_{A}\left(\gamma_{3}, \ldots, \gamma_{s} \mid\left(M / \gamma_{1} M\right) / \gamma_{2}\left(M / \gamma_{1} M\right)\right)- \\
& \epsilon_{A}\left(\gamma_{3}, \ldots, \gamma_{s} \mid\left(0:_{\left(M / \gamma_{1} M\right)} \gamma_{2}\right)\right.
\end{aligned}
$$




$$
\begin{aligned}
\epsilon_{A}\left(\gamma_{2}, \ldots, \gamma_{s} \mid\left(0:_{M} \gamma_{1}\right)\right)- & \epsilon_{A}\left(\gamma_{3}, \ldots, \gamma_{s} \mid\left(0:_{M} \gamma_{1}\right) / \gamma_{2}\left(\left(0:_{M} \gamma_{1}\right)\right)\right)- \\
& \left.e_{A}\left(\gamma_{3}, \ldots, \gamma_{s} \mid\left(0:(0)_{M} \gamma_{1}\right) \gamma_{2}\right)\right) .
\end{aligned}
$$

Esperamos com isto, que o leitor tenha percebido as dificuldades que podem surgir numa situação muito geral.

Observação 2.3.3: Se continuásscmos com o processo acima, teríamos obtido a seguinte igualdade.

$$
\begin{aligned}
e_{A}\left(\gamma_{1}, \ldots \gamma_{s} \mid M\right) & =e_{A}\left(. \mid M /\left(\gamma_{1}, \ldots, \gamma_{s}\right) M\right)-e_{A}\left(\mid\left(0: M /\left(\gamma_{1}, \ldots, \gamma_{s}\right) M\right)\right) \\
& -L_{A}\left(M /\left(\gamma_{1}, \ldots, \gamma_{s}\right) M\right)-L_{A}\left(\left(0: M /\left(\gamma_{1}, \ldots, \gamma_{s}\right) M\right)\right) .
\end{aligned}
$$

Isto mostra, que o símbolo da multiplicidade realmente cstá relacionado com o comprimento, como era de se esperar, uma vez que ele foi definido a partir do comprimento. Portanto, é natural questionar que propriedades o símbolo da multiplicidade e o comprimento possuem em comum. Discutiremos esta questão com maiores detalhes no decorrer deste capítulo.

Observação 2.3.4: ([13], pag. 331) Scjam $\gamma_{1}, \ldots, \gamma_{s}$ e $\gamma_{1}^{\prime}, \ldots, \gamma_{s}^{\prime}$ elementos de $A$ tais que $\left(\gamma_{1}, \ldots, \gamma_{s}\right)=\left(\gamma_{1}^{\prime}, \ldots, \gamma_{s}^{\prime}\right)$. Então para todo $A$-módulo Noetheriano $M$ que admite $\gamma_{1}, \ldots, \gamma_{s}$ e $\gamma_{1}^{\prime}, \ldots, \gamma_{s}^{\prime}$ como sistemas de multipliciclades tem-se que

$$
C_{A}\left(\gamma_{1}, \ldots, \gamma_{s} \mid M\right)=e_{A}\left(\gamma_{1}^{\prime}, \ldots, \gamma_{s}^{\prime} \mid M\right)
$$

As seguintes propriedades do símbolo da multiplicidade, decorrem imediatamente dà Definição 2.3.1.

i) $e_{A}\left(\gamma_{1}, \ldots, \gamma_{s} \mid M\right)$ é um numero inteiro:

ii) $\epsilon_{A}\left(\gamma_{1}, \ldots, \gamma_{s} \mid M\right)=0$ se $M=\{0\}$ :

iii) Se $M \simeq M^{\prime}$ então

$$
e_{A}\left(\gamma_{1}, \ldots, \gamma_{s} \mid M\right)=e_{A}\left(\gamma_{1}, \ldots, \gamma_{s} \mid M^{\prime}\right)
$$

cm outras palavras, o símbolo da multiplicidade é invariante por isomorfismos, isto é, quando $M$ é substituído por um módulo $M^{\prime}$ isomorfo a $M$. os símbolos da multiplicidade $e_{A}\left(\gamma_{1}, \ldots, \gamma_{s} \mid M\right)$ e $\epsilon_{A}\left(\gamma_{1}, \ldots, \gamma_{s} \mid M^{\prime}\right)$, tem o incsmo valor. 
Suponha que $M$ é um $A$-módulo Noetheriano e $\gamma_{1}, \ldots, \gamma_{s}$ é um sistema de multiplicidade em $M$. Suponha que $J$ é um ideal de $A$ tal que $J M=0$. Seja $\bar{A}=A / J$ e $\bar{\gamma}$ a imagem natural de $\gamma_{i} \mathrm{~cm} \bar{A}$. Pela Proposição $2.2 .4(i v), \bar{\gamma}_{1}, \ldots, \bar{\gamma}_{s}$ também ó um sistema de multiplicidade em $M$.

iv) (Ver [13] pag. 300) Com a notação acima

$$
e_{A}\left(\gamma_{1}, \ldots, \gamma_{s} \mid M\right)=e_{\bar{A}}\left(\overline{\gamma_{1}}, \ldots, \bar{\gamma}_{s} \mid M\right)
$$

Antes de continuarmos o nosso estudo sobre o símbolo da multiplicidade, gostaríamos de dar alguns exemplos, que embora simples, ilustram a teoria e motivam novas perguntas. Em algumas ocasiões, por razões tipográficas, omitiremos o índice do símbolo da multiplicidade $e_{A}\left(\gamma_{1}, \ldots, \gamma_{s} \mid M\right)$, escrevendo simplesmente $e\left(\gamma_{1}, \ldots, \gamma_{s} \mid M\right)$, desde que isto não cause confusão.

Exemplo 2.3.5: Seja $A=k\{x, y\}$ o anel das séries de potências sobre o corpo $k$, nas indeterminadas $x: y$. Considere o ideal $I=\left(x, y^{2}\right)$. Inicialmente note que

$$
k\{x, y\} /\left(x, y^{2}\right) \simeq k\{y\} /\left(y^{2}\right) .
$$

Segue deste isomorfismo que

$$
\begin{aligned}
L\left(k\{x, y\} /\left(x, y^{2}\right)\right) & =L\left(k\{y\} /\left(y^{2}\right)\right) \\
& \leq 2 L(k\{y\} /(y)) \\
& =2 .
\end{aligned}
$$

Na verdade, tem-se $L\left(k,\{x, y\} /\left(x, y^{2}\right)\right)=2$. Portanto, $\left\{x, y^{2}\right\}$ é um sistema de multiplicidade em $A=k\{x, y\}$

Antes de calcularmos a multiplicidade do ideal $I=\left(x, y^{2}\right)$, obscrvemos que $y$ não ć um divisor do zero em $A=k\{x, y\}$, e por isso, $(0:(k\{x, y\}) y)$ - 0 . Da mesma forma, $\left(0:\left(k\{y\} /\left(y^{2}\right)\right) x\right)=0$. Agora pela Definição 2.3.1, temos

$$
\begin{aligned}
e\left(x, y^{2} \mid k:\{x, y\}\right) & =e\left(y^{2}: k\{x, y\} /(x)\right)-e\left(y^{2} \mid(0:\{k\{x, y\}) x)\right) \\
& =e\left(y^{2} \mid k\{y\}\right) \\
& =e\left(. \mid k\{y\} /\left(y^{2}\right)\right) \quad o\left(0: k\{y\} y^{2}\right) \\
& =L\left(k\{y\} /\left(y^{2}\right)\right) \\
& =2 .
\end{aligned}
$$


Em particular, $e(x, y \mid k\{x, y\})=1$. De fato, poderíamos ainda, ter mostrado que, $e\left(x, y^{r} \mid k\{x, y\}\right)=r$, para qualquer inteiro $r>0$.

Uma questào natural que surge a partir deste excmplo é a seguinte: será que $e\left(x, y^{2} \mid k:\{x, y\}\right)=e\left(y^{2}, x \mid k\{x, y\}\right) ?$ A resposta $\{$ afirmativa. Na verdade, provaremos no final desta seção, que so $i_{1}, \ldots, i_{s}$ é uma permutação qualquer do conjunto $\{1, \ldots, s\}$, entào $e_{1}\left(\gamma_{1}, \ldots, \gamma_{s} \mid M\right)=c_{A}\left(\gamma_{i_{1}}, \ldots, \gamma_{i_{s}} \mid M\right)$.

Apesar do símbolo da multiplicidade ser um número inteiro, ainda náo temos condiçōes de provarmos que ele é $11 \mathrm{~m}$ número inteiro näo-negativo. A demonstração deste fato importante, será feita mais adiante, quando tivermos obtido outras propriedades interessantes sobre ole.

Una propriedade importante do símbolo da multiplicidade, que demonstraremos a seguir, a a propriedade aditiva. Esta propriedade é crucial para o desenvolvimento da teoria da multiplicidade, como veremos na sequéncia deste capitulo. O seguinte lema será usado na demonstraça desta importante propriedade.

Lema 2.3.6: ([13], pag, 301) Sejam

$$
0 \rightarrow M^{\prime} \rightarrow M \rightarrow M^{\prime \prime} \rightarrow 0
$$

uma sequência exata de A-módulos Noetherianos e $\gamma \in A$. Então podemos construir a seguinte sequència exata de A-módulos Noetherianos

$$
0 \rightarrow\left(0:_{M^{\prime}} \gamma\right) \rightarrow\left(0:_{M} \gamma\right) \rightarrow\left(0:_{M^{\prime \prime}} \gamma\right) \rightarrow M^{\prime} / \gamma M^{\prime} \rightarrow M / \gamma M \rightarrow M^{\prime \prime} / \gamma M^{\prime \prime} \rightarrow 0
$$

Teorema 2.3.7: Sejam $\gamma_{1}, \ldots, \gamma_{\text {s }}$ elementos do anel $A$. Suponha que

$$
0 \longrightarrow M^{\prime} \longrightarrow M \longrightarrow M^{\prime \prime} \longrightarrow 0
$$

seja uma sequência exata de $A$-módulos Noetherianos e, além disso, que $\gamma_{1}, \ldots, \gamma_{s}$ seja um sistema de multiplicidade em cada termo desta sequência. Então

$$
e_{A}\left(\gamma_{1}, \ldots, \gamma_{s} \mid M\right)=e_{A}\left(\gamma_{1} \ldots, \gamma_{s} \mid M^{\prime}\right)+e_{A}\left(\gamma_{1}, \ldots, \gamma_{s} \mid M^{\prime \prime}\right)
$$


É conveniente provar o Teorema 2.3.7, junto com o seu corolário.

Corolário 2.3.8: Seja

$$
0 \longrightarrow M_{p} \longrightarrow \cdots \rightarrow M_{1} \longrightarrow M_{0} \longrightarrow 0
$$

uma sequencia exata de $A$-módulos Noctherianos o suponha que $\gamma_{1}, \ldots, \gamma_{s}$ seja um sistema de multiplicidade em cada termo desta sequência. Então

$$
\sum_{i .0}^{p}(-1)^{i} e_{A}\left(\gamma_{1}, \ldots, \gamma_{s} \mid M_{i}\right)=0 .
$$

Demonstração: Quando $s=0$, a aditividade da função comprimento garante que o T'corema 2.3 .7 e o Corolário 2.3.8 sejam verdadeiros.

Vamos supor que $s \geq 1$ e que tanto o teorema quanto o seu corolário sejam válidos para sistemas de multiplicidades contendo apenas $s-1$ elementos. Considere então, $\gamma_{1}, \ldots, \gamma_{s}$ um sistema de multiplicidade em $M^{\prime} ; M$ e em $M^{\prime \prime}$, contendo exatamente $s$ elementos distintos.

Entrão pelo Lema 2.3.6, temos a seguinte sequência exata de $A$-módulos Noetherianos

$0 \rightarrow\left(0:_{M^{\prime}} \gamma_{1}\right) \rightarrow\left(0:_{M} \gamma_{1}\right) \rightarrow\left(0:_{M^{\prime \prime}} \gamma_{1}\right) \rightarrow M^{\prime} / \gamma_{1} M^{\prime} \rightarrow M / \gamma_{1} M \rightarrow M^{\prime \prime} / \gamma_{1} M^{\prime \prime} \rightarrow 0$

onde $\gamma_{1}$, é um elemento do sistema de multiplicidade $\gamma_{1}, \ldots, \gamma_{s}$ em $M$.

Como nesta sequência exata, cada termo é um A-módulo Noetheriano, segue pela Proposição 2.2.4 (ii) que $\gamma_{2}, \ldots, \gamma_{s}$ é um sistema de multiplicidade para cada um dos termos da sequência exata (2.4). Consequentemente estamos em posição de aplicar o Corolário 2.3.8 em (2.4). Daí temos

$$
\begin{aligned}
e_{A}\left(\gamma_{2}, \ldots, \gamma_{s} \mid M / \gamma_{1} M\right)-e_{A}\left(\gamma_{2}, \ldots, \gamma_{s} \mid\left(0:_{M} \gamma_{1}\right)\right)= & e_{A}\left(\gamma_{2}, \ldots, \gamma_{s} \mid M^{\prime} / \gamma_{1} M^{\prime}\right)- \\
& e_{A}\left(\gamma_{2}, \ldots, \gamma_{s} \mid\left(0:_{M^{\prime}} \gamma_{1}\right)\right)+ \\
& e_{A}\left(\gamma_{2}, \ldots, \gamma_{s} \mid M^{\prime \prime} / \gamma_{1} M^{\prime \prime}\right)- \\
& e_{A}\left(\gamma_{2}, \ldots, \gamma_{s} \mid\left(0:_{M^{\prime \prime}} \gamma_{1}\right)\right)
\end{aligned}
$$

Mas isto pode ser escrito, pela Definição 2.3.1, como

$$
\left.e_{A}\left(\gamma_{1}, \ldots, \gamma_{s} \mid M\right)\right)=\epsilon_{A}\left(\gamma_{1}, \ldots, \gamma_{s} \mid M^{\prime}\right)+\epsilon_{A}\left(\gamma_{1}, \ldots, \gamma_{s} \mid M^{\prime \prime}\right)
$$


Assim o teorema está provado para um sistema de multiplicidade contendo $s$ elementos.

Para provar o corolário, basta usar indução no número de elementos da sequência exatia.

Observação 2.3.9: Na Proposição 2.3.19, provaremos que se $\gamma_{1}, \ldots, \gamma_{s} \dot{e}$ um sistema de multiplicidade num A-módulo Noetheriano $M$ e para algum $i \in\{1, \ldots, s\}$, tivermos $\gamma_{i}^{m} M-0$ para algum inteiro positivo $m$, então

$$
e_{A}\left(\gamma_{1}, \ldots, \gamma_{s} \mid M\right)=0
$$

Exemplo 2.3.10: Considere o ideal $(x, y)$ do ancl $\mathbb{C}\{x, y\}$. Então $(x, y)$ pode ser visto como un submódulo de $\mathbb{C}\{x, y\}$. Assim temos a seguinte sequência exata de $\mathbb{C}\{x, y\}$-módulos Noetherianos

$$
0 \longrightarrow(x, y) \longrightarrow \mathbb{C}\{x, y\} \longrightarrow \mathbb{C}\{x, y\} /(x, y) \longrightarrow 0 .
$$

Agora, o conjunto $\left\{x, y^{2}\right\}$ é um sistema de multiplicidade no módulo $\mathbb{C}\{x, y\}$. Entrão pela Proposição 2.2.5, $\left\{x, y^{2}\right\}$ também é um sistema de multiplicidade tanto em $\{x, y\}$ quanto $\mathbb{C}\{x, y\} /(x, y)$. Mas, pela Observaçăo 2.3.9, temos que $x \mathbb{C}\{x, y\} /(x, y))=$ 0, ou seja, $e\left(x, y^{2} \mid \mathbb{C}\{x, y\} /(x, y)\right)-0$. Was pelo Exemplo 2.3.5, $e\left(x, y^{2}, \mathbb{C}\{x, y\}\right)=2$. Por outro lado,

$$
\begin{aligned}
e\left(x, y^{2} \mid(x, y)\right) & \left.-e\left(y^{2} \mid(x, y) /(x)\right)-e\left(y^{2} \mid(0):(x, y) x\right)\right) \\
& =e\left(. \mid(y) /\left(y^{2}\right)\right)-e\left(. \mid\left(0::_{(y)} y^{2}\right)\right) \\
& =L\left((y) /\left(y^{2}\right)\right) \\
& -2 .
\end{aligned}
$$

Portanto,

$$
e\left(x, y^{2} \mid \mathbb{C}\{x, y\}\right)=e\left(x, y^{2} \mid(x, y)\right)+\epsilon\left(x, y^{2} \mid \mathbb{C}\{x, y\} /(x, y)\right)
$$

Veremos mais adiante uma fórmula que possibilita o cálculo inediato da nultiplicidade de um ideal $I$, gerado por um sistema de multiplicidade da forma $\gamma_{1}^{n}, \ldots, \gamma_{*}^{n_{*}}$, onde $n_{1}, \ldots, n_{s}$, são inteiros positivos. 
Corolário 2.3.11: Sejam $M_{1}, M_{2}$ dois submódulos de um A-módulo Noctheriano $M$ e $\gamma_{1}, \ldots, \gamma_{s}$ um sistcma de multiplicidade para os $A$-módulos $M_{1}$ e $M_{2}$. Então $\gamma_{1}, \ldots, \gamma_{s}$ ć um sistema de multiplicidade para os $A$-módulos Noetherianos $M_{1}+M_{2}$ e $M_{1} \cap M_{2}$. Além disso, se $I$ é o ideal de $A$ gerado por $\gamma_{1}, \ldots, \gamma_{s}$, temos a seguinte igualdade:

$$
e_{A}\left(I \mid M_{1}+M_{2}\right)+e_{A}\left(I \mid M_{1} \cap M_{2}\right)=e_{A}\left(I \mid M_{1}\right)+\epsilon_{A}\left(I \mid M_{2}\right) .
$$

Demonstração: Inicialmente, observe que $M_{1} \cap M_{2}$ é um submódulo de $M$. Desde que $\gamma_{1}, \ldots, \gamma_{s}$ é um sistema de multiplicidade para $M_{1}$ c $M_{2}$, segue das seqüencias exatas

$$
\begin{gathered}
0 \longrightarrow M_{1} \longrightarrow M_{1}+M_{2} \longrightarrow M_{2} \longrightarrow 0 \\
0 \longrightarrow M_{1} \cap M_{2} \longrightarrow M \longrightarrow M_{2} / M_{1} \cap M_{2}
\end{gathered}
$$

c da Proposição 2.2.j, quo $\gamma_{1}, \ldots, \gamma_{s}$ também é um sistema de multiplicidade, tanto para $M_{1}+M_{2}$ quanto para $M_{1} \cap M_{2}$. Isto mostra que todos os símbolos da multiplicidade em (2.5), cstão bem definidos.

Considere agora, a seguinte sequência exata de A-módulos Noetherianos

$$
0 \longrightarrow M_{1} \longrightarrow M_{1}+M_{2} \longrightarrow\left(M_{1}+M_{2}\right) / M_{1} \longrightarrow 0 .
$$

Então do isomorfismo de $A$-módulos

$$
\left(M_{1}+M_{2}\right) / M_{2} \cong M_{2} /\left(M_{1}+M_{2}\right),
$$

temos que a sequencia

$$
0 \longrightarrow M_{1} \longrightarrow M_{1}+M_{2} \longrightarrow M_{2} /\left(M_{1}+M_{2}\right) \longrightarrow 0,
$$

é uma sequencia exata de $A$-módulos Noctherianos. Logo aplicando o 'leorema 2.3.7 na sequência exata (2.6) obtemos a igualdade

$$
e_{A}\left(\gamma_{1}, \ldots, \gamma_{s} \mid M_{1}+M_{2}\right)=e_{A}\left(\gamma_{1}, \ldots, \gamma_{s} \mid M_{1}\right)+e_{A}\left(\gamma_{1}, \ldots, \gamma_{s} \mid M_{2} /\left(M_{1}+M_{2}\right)\right)
$$

Usando o mesmo raciocínio com a seguinte sequência exata baixo

$$
() \longrightarrow M_{1} \cap M_{2} \longrightarrow M_{2}+M_{2} / M_{1} \cap M_{2} \longrightarrow 0
$$


obtemos a seguinte igualdade numórica

$$
{ }^{\prime}\left(\gamma_{1}, \ldots, \gamma_{s i} M_{2}\right)=e_{A}\left(\gamma_{1}, \ldots, \gamma_{s} M_{1} \cap M_{2}\right)+e_{A}\left(\gamma_{1}, \ldots, \gamma_{s} \mid M_{2} /\left(M_{1}+M_{2}\right)\right)
$$

Pondo em evidéncia $e_{A}\left(\gamma_{1}, \ldots, \gamma_{s} \mid M_{2} /\left(M_{1}+M_{2}\right)\right)$ em (2.8), e em seguida substituindo em (2.7), obtemos a igualdade desejada.

Teorema 2.3.12: ([13], pag. 303) Scjam $M_{1}$ e $M_{2}$ submódulos de um $A$-módulo $M$ ( $M$ não necessariamente Noetheriano) tais que $M / M_{1}$ e $M / M_{2}$ sejam Noetherianos. Se $\gamma_{1}, \ldots, \gamma_{s}$ é um sistema de multiplicidade em $M / M_{1}$ e em $M / M_{2}$, cntão é um sistema de multiplicidade tanto em $M / M_{1} \cap M_{2}$ quanto em $M /\left(M_{1}+M_{2}\right)$. Além disso, definindo $I=\left(\gamma_{1}, \ldots, \gamma_{s}\right)$ tem-se que

$$
\epsilon_{A}\left(I \mid M / M_{1} \cap M_{2}\right)+e_{A}\left(I \mid M /\left(M_{1}+M_{2}\right)\right)=e_{A}\left(I \mid M / M_{1}\right)+e_{A}\left(I \mid M / M_{2}\right) .
$$

Já observamos anteriomente, que se $M$ c $M^{\prime}$ são dois módulos isomórfos, então $\epsilon(I \mid M)=\epsilon\left(I \mid M^{\prime}\right)$. Por isso, é interessante a útil, ter algum critério que possamos utilizar no caso de dados dois módulos $M$ e $M^{\prime}$, dizer se eles são ou năo isomorfos. () próximo teorema, classicamente conhecido como o Primeiro Teorema do Isomorfismo, é uma ferramenta muito valiosa para responder a esta questão, e por isso, iremos enunciá-lo, mesmo que sem demonstração.

Teorema 2.3.13: ([13], pag. 16) Sejan $f: M \rightarrow N$ um epinorfismo de $A$-módulos, $L$ um submódulo de $N$ e $f^{\prime}(L)$ um submódulo de $M$. Então os módulos

$$
M / f^{-1}(L) \because N / L
$$

são isonorfos. Fin particular, $M / \operatorname{Ker}(f) \simeq N$. Se, além disso, $f$ for injetora, isto é, $\operatorname{Ker}(f)=\{0\}$, então $M \simeq N$.

No que segue, vamos mostrar que o símbolo da multiplicidade é invariante por permutacoes, isto 6 o valor de $e_{1}\left(\gamma_{1}, \ldots, \gamma_{s} \mid M\right)$ nào depende da ordem em que cada um dos $\gamma, i=1, \ldots, s$ aparecem no símbolo da multiplicidade. Como a prova deste 
resultado envolve vários cálculos técnicos, é mais conveniente dividirmos a sua demonstração, o que facilitará a sua compreensão. Antes de iniciarmos a demonstração, vamos fazer algumas observaçóes quanto a notaçâo. O próximo lema, pode ser considerado como o primeiro passo indutivo.

Observação 2.3.14: Scjam $K$ um A-submódulo Noetheriano de $M$ e $\gamma_{3}, \ldots$. $\gamma_{s}$ um sistema de multiplicidades cm $K$. Para representar esta afirmação, usaremos a seguinte notação.

$$
(K)-\epsilon_{A}\left(\gamma_{3}, \ldots, \gamma_{s} \mid M\right)
$$

Observação 2.3.15: Suponha que $L \subset N$ são submódulos de um $A$-módulo Noctheriano $M$ e que $\gamma_{3}, \ldots, \gamma_{s}$ seja um sistema de multiplicidade em $M / L$. Desde que as inclusões

$$
N / L \subseteq M / L
$$

são sempre verdadeiras, a seguinte sequência de $A$-módulos Noetherianos

$$
0 \longrightarrow N / L \longrightarrow M / L \longrightarrow(M / L) /(N / L) \longrightarrow 0
$$

é exata. Do isomorfismo

$$
(M / L) /(N / L) \simeq M / N
$$

segue que a seguinte sequência

$$
0 \longrightarrow N / L \longrightarrow M / L \longrightarrow M / N \longrightarrow 0
$$

também é exata. Então, pela Proposiçào $2.2 .5, \gamma 3, \ldots, \gamma$, também é um sistema do multiplicidade tanto em $N / L$ quanto em $M / N$. Além disso, pelo Teorema 2.3.7, temos

$$
(N / L)=(N / M)+(M / L)
$$

Lema 2.3.16: Sejam $M$ um $A$-módulo Noetheriano e $\gamma_{1}, \ldots, \gamma_{s}(s \geq 2)$ um sistema de multiplicidade em $M$. Então

$$
e_{A}\left(\gamma_{1}, \gamma_{2}, \ldots, \gamma_{s} \mid M\right)=e_{A}\left(\gamma_{2}, \gamma_{1}, \ldots, \gamma_{s} \mid M\right)
$$


Demonstração: Sejam $\gamma_{1}, \ldots, \gamma_{s}$ um sistema de multiplicidade $\mathrm{em} M$. Então pela Definição 2.3.1, tem-se que

$$
e_{A}\left(\gamma_{1}, \ldots, \gamma_{s} \mid M\right)=e_{A}\left(\gamma_{2}, \ldots, \gamma_{s} \mid M / \gamma_{1} M\right)-\epsilon_{A}\left(\gamma_{2}, \ldots, \gamma_{s} \mid\left(0:_{M} \gamma_{1}\right)\right)
$$

Mas, usando novamente a Definição 2.3.1, nos módulos $M / \gamma_{1} M$ e $\left(0: M / \gamma_{1} M \gamma_{2}\right)$, temos as seguintes relações

$$
\begin{aligned}
c_{A}\left(\gamma_{2}, \ldots, \gamma_{s} \mid M / \gamma_{1} M\right)= & +e_{A}\left(\gamma_{3}, \ldots, \gamma_{s} \mid\left(M / \gamma_{1} M\right) / \gamma_{1}\left(M / \gamma_{1} M\right)\right) \\
& e_{A}\left(\gamma_{3}, \ldots, \gamma_{s} \mid\left(0:_{M / \gamma_{1} M} \gamma_{2}\right)\right)
\end{aligned}
$$

$$
\begin{aligned}
-e_{1}\left(\gamma_{2}, \ldots, \gamma_{s} \mid\left(0:_{M} \gamma_{1}\right)\right)= & -e_{A}\left(\gamma_{3}, \ldots, \gamma_{s} \mid\left(0:_{M} \gamma_{1}\right) / \gamma_{2}\left(0:_{M} \gamma_{1}\right)\right)+ \\
& e_{1}\left(\gamma_{3}, \ldots, \gamma_{s} \mid\left(0:\left(0: M \gamma_{1}\right) \gamma_{2}\right)\right) .
\end{aligned}
$$

Para simplificar o nosso trabalho, vamos fazer uso da seguinte notaçāo

$$
\ell_{A}\left(\gamma_{1}, \ldots, \gamma_{s} \mid M\right)=(1)-(2)-(3)+(4)
$$

onde

$$
\begin{aligned}
& (1)=\left(\left(M / \gamma_{1} M\right) / \gamma_{2}\left(M / \gamma_{1} M\right)\right) \\
& (2)=\left(\left(0:_{M / \gamma_{1} M} \gamma_{2}\right)\right) \\
& (3)=\left(\left(0:_{M} \gamma_{1}\right) / \gamma_{2}\left(0: M \gamma_{1}\right)\right) \\
& (4)=\left(\left(0:\left(0: M \gamma_{2}\right) \gamma_{2}\right)\right) .
\end{aligned}
$$

Agora, pela Proposição 2.2.6 (iii), temos o seguinte isomorfismo de A-módulos

$$
M /\left(\gamma_{1} M+\gamma_{2} M\right) \cong\left(M / \gamma_{1} M\right) /\left(M / \gamma_{2} M\right) .
$$

Assim podemos resscrever (1) como

$$
\left.\left.(1)=\left(\left(M / \gamma_{1} M\right)\right) /\left(\gamma_{2} M / \gamma_{1} M\right)\right)\right)-\left(M /\left(\gamma_{1} M+\gamma_{2} M\right)\right) .
$$

Além disso, a igualdade abaixo

$$
\left(0:_{\left(0:{ }_{M} \gamma_{1}\right)} \gamma_{2}\right)=\left(0:_{M} \gamma_{1}\right) \cap\left(0:_{M} \gamma_{2}\right),
$$

nos permite rescrever (4) de uma mancira mais conveniente como

$$
(4)=\left(\left(0:\left(0: M \gamma_{1}\right) \gamma_{2}\right)\right)=\left(\left(0:_{M} \gamma_{1}\right) \cap\left(0:_{M} \gamma_{2}\right)\right) \text {. }
$$


Vemos com isto, que tanto (1) quanto (4) envolvem $\gamma_{1}$ e $\gamma_{2}$ simetricamente, ou seja, não importa como eles aparecem na expressão da multiplicidade, numericamente clas são iguais. Falta então provar que o mesmo acontece com a soma $(2)+(3)$.

Pcla Proposição 2.2.6 (v), t.emos

$$
\left(0:_{M / \gamma_{1} M} \gamma_{2}\right)=\left(\gamma_{1} M:_{M} \gamma_{2}\right) / \gamma_{1} M
$$

e pelas inclusões abaixo

$$
\gamma_{1} M \subseteq \gamma_{1} M+\left(0:_{M} \gamma_{2}\right) \subseteq\left(\gamma_{1} M:_{M} \gamma_{2}\right)
$$

t.emos por (2.9) que

$$
\begin{aligned}
(2) & =\left(\left(0:_{M / \gamma_{1}} \gamma_{2}\right)\right) \\
& \left.=\left(\gamma_{1} M:_{M} \gamma_{2}\right) / \gamma_{1} M\right) \\
& \left.=\left(\left(\gamma_{1} M:_{M} \gamma_{2}\right)\right) /\left(\gamma_{1} M+\left(0:_{M} \gamma_{2}\right)\right)\right)+\left(\gamma_{1} M+\left(0:_{M} \gamma_{2}\right) / \gamma_{1} M\right) \\
& \left.=\left(\left(\gamma_{1} M:_{M} \gamma_{2}\right)\right) /\left(\gamma_{1} M+\left(0:_{M} \gamma_{2}\right)\right)\right)+\left(\left(0:_{M} \gamma_{2}\right) /\left(\gamma_{1} M \cap\left(0:_{M} \gamma_{2}\right)\right)\right)
\end{aligned}
$$

pois pelo Teorema 2.3.13,

$$
\gamma_{1} M+\left(0: M \gamma_{2}\right) / \gamma_{1} M \simeq\left(0:_{M} \gamma_{2}\right) /\left(\gamma_{1} M \cap\left(0:_{M} \gamma_{2}\right)\right)
$$

Mas novamente pela Proposição $2.2 . \bar{j}(i i)$, (i), respectivamente, temos que

$$
\left(0:_{M} \gamma_{2}\right) /\left(\gamma_{1} M \cap\left(0:_{M} \gamma_{2}\right)\right)=\gamma_{1}\left(0:_{M} \gamma_{1} \gamma_{2}\right)
$$

Assim a equação (2.10) torna-so

$$
(2)=(5)+(6) \text {, }
$$

onde

$$
(5)=\left(\left(0:_{M} \gamma_{2}\right) / \gamma_{1}\left(0:_{M} \gamma_{1} \gamma_{2}\right)\right),
$$

e

$$
(6)=\left(\left(\gamma_{1} M:_{M} \gamma_{2}\right) / \gamma_{1} M+\left(0:_{M} \gamma_{2}\right)\right) .
$$


Logo temos a igualdade

$$
(1)-(2)-(3)+(4)=(1)+(4)-(5) \cdots(6) \cdot(3) \text {. }
$$

Agora a multiplicaçào por $\gamma_{2}$ fornece um epinorfismo de $A$-módulos

$$
\left(\gamma_{1} M:_{M} \gamma_{2}\right) \longrightarrow \gamma_{2}\left(\gamma_{1} M:_{M} \gamma_{2}\right)
$$

Mas pela Proposição 2.2.5 (ii), temos $\gamma_{2}\left(\gamma_{1} M:_{M} \gamma_{2}\right)-\gamma_{1} M \cap \gamma_{2} M$. Assim temos o epimorfismo de A-módulos

$$
\left(\gamma_{1} M:_{M} \gamma_{2}\right) \longrightarrow \gamma_{1} M \cap \gamma_{2} M
$$

cujo núcleo é exatamente $\left(0:_{M} \gamma_{2}\right)$ e está contido $\mathrm{cm} \gamma_{1} M+\left(0:_{M} \gamma_{2}\right)$ que é levado por esse epimorfismo em $\gamma_{1} \gamma_{2} M$. Segue, do Teorema 2.3.13, o seguinte isomorfismo

$$
\left.\left(\gamma_{1} M:_{M} \gamma_{2}\right) /\left(\gamma_{1} M+\left(0:_{M} \gamma_{2}\right)\right) \simeq\left(\gamma_{1} M \cap \gamma_{2} M\right) / \gamma_{1} \gamma_{2} M\right)
$$

Assim sendo,

$$
(6)=\left(\left(\gamma_{1} M \cap \gamma_{2} M\right) / \gamma_{1} \gamma_{2} M\right),
$$

que também é simétrico em relaçào a fo ca a $\gamma_{2}$.

Resta examinar a somal (3) + (5). As seguintes inclusôes são triviais

$$
\gamma_{2}\left(0:_{M} \gamma_{1}\right) \subseteq \gamma_{2}\left(0 \gamma_{M} \gamma_{1} \gamma_{2}\right) \subseteq\left(0:_{M} \gamma_{1}\right)
$$

Mas pela pela equação (2.9), temos

$$
(3)+(5)=(7)+\{8\}
$$

onde

$$
(7)=\left(\gamma_{2}\left(0:_{M} \gamma_{1} \gamma_{2}\right) / \gamma_{2}\left(0: M \gamma_{1}\right)\right)
$$

$$
\{8\}=\left(\left(0:_{M} \gamma_{1}\right) / \gamma_{2}\left(0{ }_{M} \gamma_{1} \gamma_{2}\right)\right)+\left(\left(0:_{M} \gamma_{2}\right) / \gamma_{1}\left(0_{M} \gamma_{1} \gamma_{2}\right)\right)
$$

A última dessas igualdades tem a propriedade de simetria desejada. Novamente, a multiplicação por $\gamma_{2}$ produz um epimorfismo

$$
\left(0: \gamma_{M} \gamma_{1}\right) \longrightarrow \gamma_{2}\left(0: M \gamma_{1} \gamma_{2}\right)
$$


cuja imagem inversa de $\gamma_{2}\left(0: M \gamma_{1}\right)$ por este epimorfismo é exatamente $\left(0::_{M} \gamma_{1}\right)+$ $\left(0:_{M} \gamma_{2}\right)$. Assim, temos pelo Teorema 2.3.13, o seguinte isomorfismo

$$
\left(0:_{M} \gamma_{1} \gamma_{2}\right) /\left(0:_{M} \gamma_{1}\right)+\left(0:_{M} \gamma_{2}\right) \cong \gamma_{2}\left(0:_{M} \gamma_{1} \gamma_{2}\right) / \gamma_{2}\left(0:_{M} \gamma_{1}\right)
$$

e portanto,

$$
(7)=\left(\left(0:_{M} \gamma_{1} \gamma_{2}\right) /\left(0:_{M} \gamma_{1}\right)+\left(0:_{M} \gamma_{2}\right)\right)
$$

que é outra expressão simétrica em relação a $\gamma_{1}$ e a $\gamma_{1}$.

Como

$$
\epsilon_{A}\left(\gamma_{1}, \ldots, \gamma_{s} M\right)=(1)+(4)-(6)-(7)-\{8\}
$$

e cada $1 \mathrm{~m}$ dos termos do lado direito tem a propricdade de permanecer inalterado quando $\gamma_{1}$ e $\gamma_{2}$ são permutados, a proposição está provada.

Proposição 2.3.17: Sejam $M$ um A-módulo Noetheriano e $\gamma_{t}, \ldots, \gamma_{s}$ um sistema de multiplicidade em $M$. Se $\left\{i_{1}, \ldots, i_{s}\right\}$ é uma permutação do conjunto $\{1, \ldots, s\}$, então

$$
e_{A}\left(\gamma_{1}, \gamma_{2}, \ldots, \gamma_{s} \mid M\right)=e_{A}\left(\gamma_{i_{1}}, \gamma_{i_{2}}, \ldots, \gamma_{i_{s}} \mid M\right)
$$

Demonstração: É suficiente mostrarmos que

$$
r_{A}\left(\gamma_{1}, \ldots, \gamma_{m}, \gamma_{m+1}, \ldots, \gamma_{s}, M\right)
$$

permanece inalterado se trocarmos $\gamma_{m}$ por $\gamma_{m+1}$.

Agora por meio de $m-1$ aplicaçóes sucessivas da Definição 2.3.1, obtemos uma expressão para $e_{A}\left(\gamma_{1}, \ldots, \gamma_{s} \mid M\right)$ como uma soma finita da forma

$$
c_{A}\left(\gamma_{1}, \ldots, \gamma_{s} \mid M\right)=\sum_{v} \epsilon_{n} e_{A}\left(\gamma_{m}, \gamma_{m+1}, \ldots, \gamma_{s} \mid M_{v}\right)
$$

onde cada $\epsilon_{v}$ é igual a \pm 1 e cada $M_{v}$ ó um A-módulo Noetheriano que admite $\gamma_{m}, \gamma_{m+1}, \ldots, \gamma_{s}$ como um sistema do multiplicidade. Além disso, os números $\epsilon_{*}$ e os módulos $M_{v}$ são determinados unicamente por $\gamma_{1}, \ldots, \gamma_{m-1}$, isto significa dizer que eles independem de $\gamma_{m}, \gamma_{m+1}, \ldots, \gamma_{s}$. Então pelo Lema 2.3 .16 , se trocarmos $\gamma_{m}$ por $\gamma_{m+1}$ obteremos

$$
e_{A}\left(\gamma_{1}, \ldots, \gamma_{m}, \gamma_{m+1}, \ldots, \gamma_{s} \mid M\right)=\sum_{\eta} \epsilon_{\eta} e_{A}\left(\gamma_{m}, \gamma_{m+1}, \ldots, \gamma_{s} \mid M_{\eta}\right)
$$


onde $\epsilon_{\eta}$ e $M_{2}$ tém o mesmo significado como antes. Mas novamente pelo Lema 2.3.16

$$
\epsilon_{A}\left(\gamma_{m}, \gamma_{m, 1}, \ldots, \gamma_{s} \mid M_{n}\right)=c_{A}\left(\gamma_{m+1}, \gamma_{m}, \ldots, \gamma_{s} \mid M_{n}\right)
$$

Segue disto que

$$
e_{1}\left(\gamma_{1}, \ldots, \gamma_{m}, \gamma_{m+1} \ldots, \gamma_{s}, M\right)=\sum_{v} \epsilon_{n} e_{A}\left(\gamma_{m}, \gamma_{m+1}, \ldots, \gamma_{s} \mid M_{n}\right)
$$

c assim a proposição está provadà.

Observação 2.3.18: 1) No Excmplo 2.3.5 havíamos questionado se os símbolos da multiplicidade $e\left(x, y^{2} \mid k[x, y]\right)$ e $\left(y^{2}, x \mid k[x, y]\right.$, eram numericamente iguais ou năo. Após a Proposição 2.3.17, podemos afirmar com certéa que eles são iguais.

2) A Proposição 2.3.17, também é conhecida como "Propriedade da Mudança", e clesempenhará um papel importante em algumas demonstraçôes no decorrer desta seção. A próxima proposição é uma aplicação imediata dela.

Proposição 2.3.19: Sejam $M$ um A-módulo Noetheriano e $\gamma_{1}, \ldots, \gamma_{s}$ um sistema de: multiplicidade em $M$. Suponha que para algum valor particular de $i \in\{1, \ldots, s\}$ tenhamos $\gamma_{i}^{m} M=0$, onde $m$ ć um inteiro positivo. Entäo

$$
e_{A}\left(\gamma_{1}, \ldots, \gamma_{s} \mid M\right)=0
$$

Demonstração: $\Lambda$ Proposição 2.3 .17 mostra que podemos supor que $i=1$. Tendo feito esta hipótese adicional, provaremos a proposição por indução sobre $m$.

Primeiro suponha que $m=1$. Então $\gamma_{1} M=0$, e portanto, $M / \gamma_{1} M \cong M$ e $\left(0:_{M} \gamma_{1}\right)=M$. Assim, pela Definição 2.3.1, temos

$$
e_{A}\left(\gamma_{1}, \ldots, \gamma_{s}, M\right)=\epsilon_{A}\left(\gamma_{2}, \ldots, \gamma_{s} \mid M\right) \cdots \iota_{A}\left(\gamma_{2}, \ldots, \gamma_{s} \mid M\right)=0
$$

Agora vamos supor que $m>1$, o que o resultado descjado já foi provado para valores menores da variável indutiva, isto é, para $m-1$. Pelo Teorema 2.3.7 e pela sequência exata,

$$
0 \rightarrow \gamma_{1} M \longrightarrow M \longrightarrow M / \gamma_{1} M \longrightarrow 0
$$


temos quo

$$
e_{A}\left(\gamma_{1}, \ldots, \gamma_{s} \mid M\right)=e_{A}\left(\gamma_{1}, \ldots, \gamma_{s} \mid \gamma_{1} M\right)+e_{A}\left(\gamma_{1}, \ldots, \gamma_{s} \mid M / \gamma_{1} M\right)
$$

Porém $\gamma_{1}^{m-1}\left(\gamma_{1} M\right)=0$ e $\gamma_{1}\left(M / \gamma_{1} M\right)=0$. Assim pela nossa hipótese indutiva, tanto ${ }^{\prime}{ }_{A}\left(\gamma_{1}, \ldots, \gamma_{s} \mid \gamma_{1} M\right)$ quanto $e_{A}\left(\gamma_{1}, \ldots, \gamma_{s} \mid M / \gamma_{1} M\right)$ são ambos igualis a zero.

Portanto

$$
\epsilon_{A}\left(\gamma_{1}, \ldots, \gamma_{s} M\right)-0
$$

como queríamos demonstrar.

\subsection{Relação entre Multiplicidade e Comprimento}

Sejarm $M$ um $A$-módulo Noetheriano o $\gamma_{1}, \ldots, \gamma_{s}$ um sistema de multiplicidade em $M$. Nesta seção iremos procurar relaçoes entre a multiplicidade e $\left(\gamma_{1}, \ldots, \gamma_{s} \mid M\right)$ o o comprimento $L\left(M /\left(\gamma_{1}, \ldots, \gamma_{s}\right) M\right)$. No caso de um anel $A$, podemos adiantar o seguinte resultado.

Teorema 2.4.1: ([9], pag, 138) Num anc] local Noctheriano $(A, \mathcal{M})$, são equivalentes:

i) $A$ é um and Cohen-Macaulay;

ii) $\mathrm{L}(\mathrm{A} / \mathrm{I})=\mathrm{e}(\mathrm{I}, \mathrm{A})$, para qualquer ideal parâmetro $I$ de $A$ :

iii) $\mathrm{L}(\mathrm{A} / \mathrm{I})=\mathrm{e}(\mathrm{I}, \mathrm{A})$, para algum ideal parâmetro $I$ de $A$.

Observação 2.4.2: ([9], pags. 109) Vamos observar que se $(A, \mathcal{M})$ a um anel local $d$-dimensional e $x_{1}, \ldots, x_{d}$ ć um sistema de parâmetros de $A c I=\left(x_{1}, \ldots, x_{d}\right)$ então

$$
L_{A}(A / I)>C_{A}(I \mid A)
$$

Portianto, o Teorema 2.4 .1 mostra que a igualdade ocorre se, somente se, o anel é Cohen-Macaulay.

No caso de um $A$-módulo $M$, com $\operatorname{dim} A>0$, temos um resultado semelhante ao Teorema 2.4 .1 
Mas como já observamos, um sistema de multiplicidade pode não ser um sistema de parâmetros a menos que consideremos anéis Voetheriano que também sejam locais. Vamos voltar ao caso em que nào exigimos que $A$ seja local.

Lema 2.4.3: Seja $M$ um A-módulo Noetheriano e $\gamma$ um elemento de $A$. Seja $F_{m}=$ $M /\left(0: M \gamma^{m}\right)$, então $\left(0: f_{m} \gamma\right)=0$ desde que $m$ seja um inteiro suficientemente grande.

Demonstração: Desde que $M$ ć um $A$-módulo Noetheriano, a sequência ascendente de submódulos de $M$

$$
\left(0: i_{M} \gamma_{1}\right) \subseteq\left(0: M \gamma_{1}^{2}\right) \subset\left(0:_{M} \gamma_{1}^{3}\right) \subseteq \ldots
$$

deve ser estacionárial.

Suponhamos que $m$ é suficientemente grande para garantir que

$$
\left(0:_{M} \gamma_{1}^{m}\right)=\left(0: M \gamma_{1}^{m+1}\right)
$$

Fintão, pcla Proposição $2.2 .5(v)$ e $(i)$, temos as igualdades

$$
\begin{aligned}
\left(0: F_{m} \gamma\right) & =\left(\left(0:_{M} \gamma_{1}^{m}\right):_{M} \gamma\right) /\left(\left(0:_{M} \gamma_{1}^{m}\right)\right) \\
& =\left(0:_{M} \gamma_{1}^{m-1}\right) /\left(0:_{M} \gamma_{1}^{m}\right) \\
& =\left(0:_{M} \gamma_{1}^{m}\right) /\left(0:_{M} \gamma_{1}^{m}\right) \\
& =0
\end{aligned}
$$

como queríamos demonstrar

O próximo teorema, mostra que dados um sistema de nultiplicidade, $\gamma_{1}, \ldots, \gamma_{s}$ de um A-módılo $M$, então a multiplicidade $e_{A}\left(\gamma_{1}, \ldots, \gamma_{s} M\right)$ é limitada, inferiormente pelo 0 e superiormente pelo inteiro não-negativo $L_{A}\left(M / \gamma_{1} M+\ldots+\gamma_{s} M\right)$. Observamos que não estamos dizendo que o símbolo da multiplicidade é uma função limitada no conjunto de todos os sistemas de multiplicidades do anel $A$.

Teorema 2.4.4: Scjam $M$ um A-módulo Noetheriano e $\gamma_{1}, \ldots, \gamma_{s}$ um sistema de multiplicidade em $M$. Então

$$
0 \leq e_{A}\left(\gamma_{1}, \ldots, \gamma_{s} \mid M\right) \leq L_{A}\left(M / \gamma_{1} M+\ldots+\gamma_{s} M\right) .
$$


Demonstração: Para provarmos quo

$$
e_{A}\left(\gamma_{1}, \ldots, \gamma_{s} \mid M\right) \geq 0
$$

usaremos indução sobre o número de clementos no sistema de multiplicidade.

Se $s=0$, então $e_{A}(. \mid M)=L_{A}(M) \geq 0$, pela definição do símbolo de multiplicidade e pelo fato que o comprimento é sempre um número inteiro não-negativo.

Portanto assuma que $s \geq 1$ e que o símbolo de multiplicidade ć um intciro nãonegativo para qualquer sistema de multiplicidades consistindo apenas de $s-1$ elementos.

Seja $F=M /\left(0:_{M} \gamma_{1}^{m}\right)$, onde $m$ foi escolhido suficientemente grande para assegurar que $\left(0: F \gamma_{1}\right)=0$, o que é possível pelo Lema 2.4 .3 .

Em scguida, aplicando o Teorema 2.3.T na sequência exata abaixo

$$
0 \longrightarrow\left(0:_{M} \gamma_{1}^{m}\right) \longrightarrow M \longrightarrow F \longrightarrow 0
$$

temos que

$$
e_{A}\left(\gamma_{1}, \ldots, \gamma_{s} \mid M\right)=e_{A}\left(\gamma_{1}, \ldots, \gamma_{s} \mid F\right)+e_{A}\left(\gamma_{1}, \ldots, \gamma_{s} \mid\left(0: M \gamma_{1}^{m}\right)\right)
$$

Comı $\gamma_{1}^{m}\left(0:_{M} \gamma_{1}^{m}\right)=0$, segue da Proposição 2.3.19, que $e_{A}\left(\gamma_{1}, \ldots, \gamma_{s} \mid\left(0:_{M} \gamma_{1}^{m}\right)\right)=0$. Por outro lado, termos

$$
\begin{aligned}
e_{A}\left(\gamma_{1}, \ldots, \gamma_{s} \mid F\right) & =e_{A}\left(\gamma_{2}, \ldots, \gamma_{s} \mid F^{\prime} / \gamma_{1} F\right)+e_{A}\left(\gamma_{2}, \ldots, \gamma_{s} \mid\left(0: \gamma_{1}\right)\right) \\
& =e_{A}\left(\gamma_{2}, \ldots, \gamma_{s} \mid F / \gamma_{1} F\right)
\end{aligned}
$$

pois $\gamma_{1}\left(0:{ }_{F} \gamma_{1}\right)=0$. Portanto das cquaçöes 2.12 e 2.13 , temos

$$
e_{A}\left(\gamma_{1}, \ldots, \gamma_{s} \mid M\right)=e_{A}\left(\gamma_{2}, \ldots, \gamma_{s} \mid F / \gamma_{1} F\right)
$$

Mas pela hipótese indutiva, como $\gamma_{2}, \ldots, \gamma_{s}$ é um sistema de multiplicidade contendo s-1 clementos, obtemos a seguinto desigualdade

$$
e_{A}\left(\gamma_{2}, \ldots, \gamma_{s} \mid F / \gamma_{1} F\right) \geq 0
$$

ou seja,

$$
s_{A}\left(\gamma_{1}, \ldots, \gamma_{s} \mid M\right) \geq 0
$$


e isto prova a primeira desigualdade.

Vamos estabelecer a segunda desigualdade. Se $s \geq 1$ 1, cmos, pela Definição 2.3.1, que

$$
\epsilon_{A}\left(\gamma_{1}, \ldots, \gamma_{s} \mid M\right)=\epsilon_{A}\left(\gamma_{2}, \ldots, \gamma_{s} \mid M / \gamma_{1} M\right) \cdot c_{A}\left(\gamma_{2}, \ldots, \gamma_{s} \mid\left(0:_{M} \gamma_{1}\right)\right)
$$

ou seja,

$$
e_{A}\left(\gamma_{1}, \ldots, \gamma_{s} \mid M\right) \leq \epsilon_{A}\left(\gamma_{1}, \ldots, \gamma_{s} \mid M / \gamma_{1} M\right)
$$

pois $e_{A}\left(\gamma_{1}, \ldots, \gamma_{s} \mid\left(0:_{M} \gamma_{1}\right)\right) \geq 0$, pelo que acabamos do provar acima.

Seja $M^{\prime}=M / \gamma_{1} M$. Segue pelo mesmo argumento anterior, que

$$
e_{A}\left(\gamma_{1}, \ldots, \gamma_{s} \mid M\right) \leq \epsilon_{A}\left(\gamma_{2}, \ldots, \gamma_{s} \mid M^{\prime}\right) \leq \epsilon_{A}\left(\gamma_{3}, \ldots, \gamma_{s} \mid M^{\prime} / \gamma_{2} M^{\prime}\right) .
$$

Mas pela Proposição 2.2.5 (iii) temos que.

$$
M /\left(\gamma_{1} M+\gamma_{2} M\right) \cong\left(M / \gamma_{1} M\right) / \gamma_{2}\left(M / \gamma_{1} M\right)=M^{\prime} / \gamma_{2} M^{\prime}
$$

Portanto

$$
\epsilon_{A}\left(\gamma_{1}, \ldots, \gamma_{s} \mid M\right) \leq e_{A}\left(\gamma_{3}, \ldots, \gamma_{s} \mid M /\left(\gamma_{1} M+\gamma_{2} M\right)\right)
$$

Procedendo desta maneira teremos

$$
\begin{aligned}
e_{A}\left(\gamma_{1}, \ldots, \gamma_{s} \mid M\right) & \leq e_{A}\left(\mid M / \gamma_{1} M+\ldots+\gamma_{s} M\right) \\
& -L_{A}\left(M / \gamma_{1} M+\ldots+\gamma_{s} M\right),
\end{aligned}
$$

e assim o tcorema está provado.

Corolário 2.4.5: Se $\gamma_{1}, \ldots, \gamma_{s}$ é um sistema de multiplicidade num $A$-módulo Noetheriano $M$ tal que $\gamma_{1} M+\ldots+\gamma_{s} M=M$. Então

$$
e_{A}\left(\gamma_{1}, \ldots, \gamma_{s} \mid M\right)=0
$$

Demonstração: P'elo 'l'eorema 2.4.4.

$$
0 \leq \epsilon_{A}\left(\gamma_{1}, \ldots, \gamma_{s} \mid M\right) \leq L_{A}\left(M / \gamma_{1} M+\ldots+\gamma_{s} M\right)
$$

Mas como, por hipótese, $\gamma_{1} M+\ldots+\gamma_{s} M=M$, então

$$
\left(M / \gamma_{1} M+\ldots+\gamma_{s} M\right) \cong\{0\}
$$


Portanto, $e_{A}\left(\gamma_{1}, \ldots, \gamma_{s} \mid M\right)=0$

O próximo teorema mostra que o símbolo da multiplicidade comporta-se como uma aplicação aditiva no conjunto dos sistemas de multiplicidades do anel $A$.

Teorema 2.4.6: Sejam $M$ um A-módulo Noetheriano $\gamma_{1}, \ldots, \gamma_{i}, \ldots, \gamma_{s}$ e $\gamma_{1}, \ldots, \gamma_{i}^{\prime}, \ldots, \gamma_{s}$ dois sistemas de multiplicidades em $M$. Então $\gamma_{1}, \ldots, \gamma_{i} \gamma_{i}^{\prime}, \ldots, \gamma_{s}$ também é um sistema de multiplicidade $\mathrm{em} M$. Além disso,

$$
\left.e_{A}\left(\gamma_{1}, \ldots, \gamma_{i} \gamma_{i}^{\prime}, \ldots, \gamma_{s} \mid M\right)=e_{A}\left(\gamma_{1}, \ldots, \gamma_{i}, \ldots, \gamma_{s}\right) \mid M\right)+e_{A}\left(\gamma_{1}, \ldots, \gamma_{i}^{\prime}, \ldots, \gamma_{s} \mid M\right)
$$

Demonstraçāo: Seja $F^{\prime}=\gamma_{1} M+\ldots+\gamma_{i} M+\ldots+\gamma_{s} M$. Então $F$ é um submódulo de $M$ e portanto, pela Proposição 2.2.5, $\gamma_{1}, \ldots, \gamma_{i}^{\prime}, \ldots, \gamma_{s}$ é um sistema de multiplicidade em $F$.

Das inclusões abaixo

$$
\gamma_{1} F+\ldots+\gamma_{i}^{\prime} F+\ldots+\gamma_{s} F \subseteq F \subseteq M
$$

temos pela Observação 2.3 .15

$L_{A}\left(M / \gamma_{1} F+\ldots+\gamma_{i}^{\prime} F+\ldots+\gamma_{s} F\right)=L_{A}(M / F)+L_{A}\left(F / \gamma_{1} F+\ldots+\gamma_{i}^{\prime} F+\ldots+\gamma_{s} F\right)$,

e nesta equação, os termos do lado direito são finitos. Das inclusões abaixo

$$
\gamma_{1} F+\ldots+\gamma_{i}^{\prime} F+\ldots+\gamma_{s} F \subset \gamma_{1} M+\ldots+\gamma_{i} \gamma_{i}^{\prime} M+\ldots+\gamma_{s} M \subseteq M
$$

obtemos a seguinte igualdade numérica

$$
\begin{aligned}
& L_{A}\left(M / \gamma_{1} F+\ldots+\gamma_{i}^{\prime} F+\ldots+\gamma_{s} F\right)=L_{A}\left(M / \gamma_{1} M+\ldots+\gamma_{i} \gamma_{i}^{\prime} M+\ldots+\gamma_{s} M\right) \\
& +L_{A}\left(\left(\gamma_{1} M+\ldots+\gamma_{i} \gamma_{i}^{\prime} M+\ldots+\gamma_{s} M\right) /\left(\gamma_{1} F+\ldots+\gamma_{i}^{\prime} F+\ldots+\gamma_{s} F\right)\right),
\end{aligned}
$$

e como o comprimento só assume valores não negativos, segue que

$$
L_{A}\left(M / \gamma_{1} M+\ldots+\gamma_{i} \gamma_{i}^{\prime} M+\ldots+\gamma_{s} M\right) \leq \infty
$$


Portanto, $\gamma_{1}, \ldots, \gamma_{i} \gamma_{i}^{\prime}, \ldots, \gamma_{s}$ é, por definiçăo, um sistema de multiplicidade em M. Vamos provar a equação (2.14) observando, que em vista da Proposição 2.3.17 podemos supor que $i=s$. Por aplicaçoes sucessivas da Definição 2.3.1, obtemos

$$
e_{A}\left(\gamma_{1}, \ldots, \gamma_{i} \gamma_{i}^{\prime}, \ldots, \gamma_{s} \mid M\right)=\sum_{v} \kappa_{v} e_{A}\left(\gamma_{s} \mid M_{v}\right)
$$

onde o lado direito acima é uma soma finita e cada c -+1 o $M_{v}$ são A-móslulos Northerianos para os cuais $\gamma$ ś um sistema de multiplicidade. Além disso, os números $\epsilon_{v}$ e $M_{v}$ dependem somente de $M$ e dos elementos $\gamma_{1}, \ldots, \gamma_{s-1}$. Segue que

$$
e_{A}\left(\gamma_{1}, \ldots, \gamma_{s} 1, \gamma_{s}^{\prime} \mid M\right)-\sum_{n} c_{v} e_{A}\left(\gamma_{s}^{\prime} \mid M_{v}\right)
$$

$$
\epsilon_{4}\left(\gamma_{1}, \ldots, \gamma_{s-1}, \gamma_{s} \gamma_{s}^{\prime} \mid M\right)=\sum_{v} \epsilon_{\eta} \epsilon_{A}\left(\gamma_{s} \gamma_{s}^{\prime} \mid M_{\eta}\right)
$$

com os mesinos ce $c_{v}$ de antes.

A partir das duas igualdades acima, vemos que basta provarmos o teorema no caso em que $s=1$. No que segue, assumiremos que temos esta hipótese adicional a simplificaremos a notação, escrevendo $\gamma=\gamma_{s}$ e $\gamma^{\prime}-\gamma_{s}^{\prime}$.

Pela Definição 2.3.1, temos

$$
\begin{gathered}
c_{A}(\gamma / M)-L_{A}(M / \gamma M) \cdots L_{A}\left(\left(0:_{M} \gamma\right)\right) \\
c_{A}\left(\gamma^{\prime} / M\right)-L_{A}\left(M / \gamma^{\prime} M\right) \cdots L_{A}\left(\left(0: M \gamma^{\prime}\right)\right) \\
e_{A}\left(\gamma \gamma^{\prime} / M\right)=L_{A}\left(M / \gamma \gamma^{\prime} M\right)-L_{A}\left(\left(0:_{M} \gamma \gamma^{\prime}\right)\right) .
\end{gathered}
$$

Agora, a multiplicasão por $\gamma$, produr um epimorfismo

$$
M-\gamma M
$$

com a propriedade que a imagem inversa de $\gamma \gamma^{\prime} M$ ce exatamente $(0: M \gamma)+\gamma^{\prime} M$. Assim temos o isomorfismo

$$
\gamma M / \gamma \gamma^{\prime} M \cong M /\left((0: M \gamma)+\gamma^{\prime} M\right)
$$

Das inclusoes

$$
\gamma^{\prime} M \subseteq(0: M \gamma)+\gamma^{\prime} M \subseteq M
$$


temos a igualdade

$$
\left.L_{A}\left(M / \gamma^{\prime} M\right)=L_{A}\left(M /\left(0:_{M} \gamma\right)+\gamma^{\prime} M\right)+L_{A}\left(\left(0:_{M} \gamma\right)+\gamma^{\prime} M\right) / \gamma^{\prime} M\right),
$$

mas do isomorfismo (2.15), obtemos a seguinte igualdade

$$
L_{A}\left(\gamma M / \gamma \gamma^{\prime} M\right)=L_{A}\left(M / \gamma^{\prime} M\right)-L_{A}\left(\left(\left(0:_{M} \gamma\right)+\gamma^{\prime} M\right) / \gamma^{\prime} M\right)
$$

Porém valem das seguintes relações

$$
\left(\left(0:_{M} \gamma\right)+\gamma^{\prime} M\right) / \gamma^{\prime} M \cong\left(0:_{M} \gamma\right) /\left(\gamma^{\prime} M \cap\left(0:_{M} \gamma\right)\right)=\left(0:_{M} \gamma\right) / \gamma^{\prime}\left(0:_{M} \gamma \gamma^{\prime}\right)
$$

obtemos a seguinte igualdade

$$
L_{A}\left(\left(\left(0:_{M} \gamma\right)+\gamma^{\prime} M\right) / \gamma^{\prime} M\right)=L_{A}\left(\left(0:_{M} \gamma\right)\right)-L_{A}\left(\gamma^{\prime}\left(0:_{M} \gamma \gamma^{\prime}\right)\right),
$$

c assim, (1.16) torna-se

$$
L_{A}\left(\gamma M / \gamma \gamma^{\prime} M\right)=L_{A}\left(M / \gamma^{\prime} M\right)-L_{A}\left(0:_{M} \gamma\right)+L_{A}\left(\gamma^{\prime}\left(0:_{M} \gamma \gamma^{\prime}\right)\right)
$$

Além disso, somando-se $L_{A}(M / \gamma M)$ a ambos os lados da igualdade acima, obtemos

$$
L_{A}\left(M / \gamma \gamma^{\prime} M\right)=L_{A}(M / \gamma M)-L_{A}\left(0 i_{M} \gamma\right)+L_{A}\left(M / \gamma^{\prime} M\right)+L_{A}\left(\gamma^{\prime}\left(0:_{M} \gamma \gamma^{\prime}\right)\right) .
$$

Para completar a prova será suficionte mostrar que

$$
L_{A}\left(\gamma^{\prime}\left(0:_{M} \gamma \gamma^{\prime}\right)\right)=L_{\Lambda}\left(0:_{M} \gamma \gamma^{\prime}\right)-L_{A}\left(0:_{M} \gamma^{\prime}\right)
$$

Mas isto é verdade, pois a multiplicação por $\gamma^{\prime}$ produz um epimorfismo

$$
\left(0:_{M} \gamma^{\prime}\right) \longrightarrow \gamma^{\prime}\left(0:_{M} \gamma^{\prime}\right)
$$

cujo núcleo é exatamente $\left(0:_{M} \gamma^{\prime}\right)$. Daí pelo Teorema 2.3.13, tomos o seguinte isomorfismo

$$
\left(0:_{M} \gamma \gamma^{\prime}\right) /\left(0:_{M} \gamma^{\prime}\right) \simeq \gamma^{\prime}\left(0:_{M} \gamma \gamma^{\prime}\right)
$$

Por outro lado, a seguinte sequência exata

$$
0 \longrightarrow\left(0:_{M} \gamma^{\prime}\right) \longrightarrow\left(0:_{M} \gamma \gamma^{\prime}\right) \longrightarrow\left(0:_{M} \gamma \gamma^{\prime}\right) /\left(0:_{M} \gamma^{\prime}\right) \longrightarrow 0
$$


nos fornece a igualdade

$$
L_{A}\left(0:_{M} \gamma \gamma^{\prime}\right)=L_{\Lambda}\left(0:_{M} \gamma^{\prime}\right)+L_{A}\left(\left(0:_{M} \gamma \gamma^{\prime}\right) /\left(0:_{M} \gamma^{\prime}\right)\right),
$$

c disto obtemos novamente a partir do Teorema 2.3 .13 o seguinte isomorfismo

$L_{A}\left(M / \gamma^{\prime} M\right)-L_{A}\left(0:_{M} \gamma \gamma^{\prime}\right)=L_{A}(M / \gamma M)-L_{A}\left(0:_{M} \gamma\right)+L_{A}\left(M / \gamma^{\prime} M\right)-L_{A}\left(0:_{M} \gamma^{\prime}\right)$

ou seja,

$$
e_{A}\left(\gamma \gamma^{\prime} \mid M\right)=e_{A}(\gamma \mid M)+e_{A}\left(\gamma^{\prime} \mid M\right)
$$

como queríanos demonstrar.

() Teorema 2.4.6, permite resolvermos o Exemplo 2.3.5, mais rapidamente, sem termos que nos envolver em cálculos que muitas vezes são trabalhosos. Basta ver que $\{x, y\}$ ć um sistema de multiplicidade em $\mathbb{C}[x, y])$ e portanto, pelo Teorema 2.4.6, $\left\{x, y^{2}\right\}$ também. Logo temos,

$$
e\left(x, y^{2} \mid \mathbb{C}[x, y]\right)=e(x, y \mid \mathbb{C}[x, y])+e(x, y \mid \mathbb{C}[x, y])=2 .
$$

Corolário 2.4.7: Sejam $M$ um A-módulo Noctheriano, $\gamma_{1}, \ldots, \gamma_{s}$ um sistema de multiplicidado $\mathrm{cm} M$ e $n_{1}, \ldots, n_{\mathrm{s}}$ inteiros positivos. Entào

$$
\epsilon_{A}\left(\gamma_{1}^{n_{1}}, \ldots, \gamma_{s}^{n_{s}} \mid M\right)=n_{1} \cdots n_{s} e_{A}\left(\gamma_{1}, \ldots, \gamma_{s} \mid M\right)
$$

Demonstração: Basta usar o Teorerna 2.4.6 sucessivamente.

Exemplo 2.4.8: O Corolário 2.4.7 permite concluir imediatamente que

$$
\Theta\left(x^{2}, y \mid \mathbb{C}[x, y]\right)=2 \text {. }
$$

Observe que pelo Teorema 2.4.6, $x_{1}^{n 1}, \ldots, x_{s}^{n_{s}}$, ć um sistema de multiplicidades em $\mathbb{C}\left[x_{1}, \ldots, x_{s}\right]$. Podemos então calcular facilmente a multiplicidade do ideal gerado pelo elementos $x_{1}^{n_{1}}, \ldots, x_{s}^{n_{s}}$. De fat.o, pelo Corolário 2.4.7,

$$
e\left(x_{1}^{n:}, \ldots, x_{s}^{n_{s}} \mid \mathbb{C}\left[x_{1}, \ldots, x_{s}\right]\right)-n_{1} \cdots n_{s}
$$


Corolário 2.4.9: Scjam $M$ um A-módulo Noctheriano e $\gamma_{1}, \ldots, \gamma_{s}$ um sistema de multiplicidade em $M$. Então

$$
0 \leq \epsilon_{A}\left(\gamma_{1}, \ldots, \gamma_{s} \mid M\right) \leq \frac{L_{A}\left(M / \gamma_{1}^{n_{1}} M+\ldots+\gamma_{s}^{n_{s}} M\right)}{n_{1} \cdots n_{s}},
$$

para intciros positivos arbitrários $n_{1}, \ldots, n_{s}$.

Demonstração: Pelos Corolário 2.4.7 e Teorema 2.4.4, temos

$$
0 \leq n_{1} \cdots n_{s} e_{A}\left(\gamma_{1}, \ldots, \gamma_{s} \mid M\right)=e_{A}\left(\gamma_{1}^{n_{1}}, \ldots, \gamma_{s}^{n_{*}} \mid M\right) \leq L_{A}\left(M / \gamma_{1}^{n_{1}} M+\ldots+\gamma_{s}^{n_{s}} M\right),
$$

pois $\gamma_{1}^{n_{1}} \ldots, \gamma_{s}^{n_{s}}$ lambém ó um sistema de multiplicidade em $M$.

Dividindo por $n_{1} \cdots n_{s}$, obtemos

$$
0 \leq e_{A}\left(\gamma_{1}, \ldots, \gamma_{s} \mid M\right) \leq \frac{L_{A}\left(M / \gamma_{1}^{n_{1}} M+\ldots+\gamma_{s}^{n_{s}} M\right)}{n_{1} \cdots n_{s}},
$$

como queríamos provar.

Corolário 2.4.10: Scjam $M$ um A-módulo Voetheriano e $\gamma_{1}, \ldots, \gamma_{s}$ um sistema de multiplicidade en $M$. Suponha que

$$
\gamma_{i}^{m} M \subseteq \gamma_{1} M+\ldots+\gamma_{i \cdot 1} M+\gamma_{i 11} M+\ldots+\gamma_{s} M
$$

onde $m$ ć um inteiro positivo. Então $e_{A}\left(\gamma_{1}, \ldots, \gamma_{s} \mid M\right)=0$.

Demonstração: Pela Proposição 2.3.17, podemos supor que $i=1$. Se $n>m$, então

$$
\gamma_{1}^{n} M+\gamma_{2} M+\ldots+\gamma_{s} M=\gamma_{2} M+\ldots+\gamma_{s} M,
$$

e assim pelo Corolário 2.4.10,

$$
0 \leq n e_{A}\left(\gamma_{1}, \ldots, \gamma_{s} \mid M\right) \leq L_{A}\left(M / \gamma_{1}^{n} M+\ldots+\gamma_{s} M\right)-L_{A}\left(M / \gamma_{2} M+\ldots+\gamma_{s} M\right) .
$$

Como $\gamma_{1}, \ldots, \gamma_{s}$ é um sistema de multiplicidades $\mathrm{m} M, \gamma_{1}^{n_{1}}, \ldots, \gamma_{s}^{n_{s}}$ também ć um sistema de multiplicidades em $M$. Logo temos que $L_{A}\left(M / \gamma_{2} M+\ldots+\gamma_{s} M\right)$ é finito. Portanto, dividindo por $n$ e fazendo $n$ tender ao infinito, o resultado segue.

Já sabemos que se $M$ é um A-módulo Noetheriano, então valem as desigualdades

$$
0 \leq e_{A}\left(\gamma_{1}, \ldots, \gamma_{N} \mid M\right)<L_{A}\left(M / \gamma_{1} M+\ldots+\gamma_{s} M\right) .
$$


Vaturalmente, segue da definição do multiplicidade, que se $s=0$, então a igualdade ć scmpro verdadeira. Gostaríamos entăo, que dado um sistema de multiplicidade $\gamma_{1}, \ldots, \gamma_{s}$, com $s>0$, obter condiçoss sobre ele, de modo que tivéssemos a igualdade entre a multiplicidade $e_{A}\left(\gamma_{1}, \ldots, \gamma_{s} M\right)$ e o comprimento $L_{A}\left(M / \gamma_{1} M+\ldots+\gamma_{s} M\right)$. O teorema abaixo, dá uma condição suficiente para esta questão.

Teorema 2.4.11: Scjam $M$ um A-módulo Noetheriano e $\gamma_{1} \ldots, \gamma_{s}$ um sistema de multiplicidade em $M$. So

$$
\left(\left(\gamma_{1} M+\ldots+\gamma_{i} M\right):_{M} \gamma_{i+1}\right)=\left(\gamma_{1} M+\ldots+\gamma_{i} M\right)
$$

para $0 \leq i \leq s-1$, então

$$
e_{A}\left(\gamma_{1}, \ldots, \gamma_{s} \mid M\right)=L_{A}\left(M / \gamma_{1} M+\ldots+\gamma_{s} M\right)
$$

Demonstração: Seja $M_{i}-M /\left(\gamma_{1} M+\ldots+\gamma_{i} M\right)$. Então pela Proposição 2.2 .6 (v), temos

$$
\begin{aligned}
\left(0:_{M_{i}} \gamma_{i+1}\right) & =\left(0:_{M /\left(\gamma_{1} M+\ldots \cdot \gamma_{i} M\right)} \gamma_{i+1}\right) \\
& \left.=\left(\left(\gamma_{1} M+\ldots+\gamma_{i} M\right):_{M} \gamma_{i+1}\right)\right) /\left(\gamma_{1} M+\ldots+\gamma_{i} M\right) \\
& \cong 0
\end{aligned}
$$

pois por hipótese

$$
\left(\left(\gamma_{1} M+\ldots+\gamma_{i} M\right):_{M} \gamma_{i+1}\right)=\left(\gamma_{1} M+\ldots+\gamma_{i} M\right)
$$

para $0 \leq i \leq s-1$. Em particular, quando $i=0 .\left(0:_{M} \gamma_{1}\right)=0$. Agora, pola Proposição $2.2 .6($ iii $), M_{i} / \gamma_{i+1} M_{i}$ e $M_{i \div 1}$ são isomórficos, pois

$$
\begin{aligned}
M_{i} / \gamma_{i+1} M_{i} & =\left(M /\left(\gamma_{1} M+\ldots+\gamma_{s}\right)\right) /\left(\gamma_{i+1}\left(M /\left(\gamma_{1} M+\ldots+\gamma_{s}\right)\right)\right. \\
& \cong M / \gamma_{1} M+\ldots+\gamma_{i+1} M \\
& =M_{i+1} .
\end{aligned}
$$


Assim sendo, como $\left(0:_{M} \gamma_{1}\right)=0$, tem-se

$$
\begin{aligned}
e_{A}\left(\gamma_{1}, \ldots, \gamma_{s} \mid M\right) & =e_{A}\left(\gamma_{2}, \ldots, \gamma_{s} \mid M / \gamma_{1} M\right)-e_{A}\left(\gamma_{2}, \ldots, \gamma_{s} \mid\left(0:_{M} \gamma_{1}\right)\right) \\
& =e_{A}\left(\gamma_{2}, \ldots, \gamma_{s} \mid M / \gamma_{1} M\right) \\
& =e_{A}\left(\gamma_{2}, \ldots, \gamma_{s} \mid M M_{1}\right) \\
& =e_{A}\left(\gamma_{2}, \ldots, \gamma_{s} \mid M_{1} / \gamma_{2} M_{1}\right)-\epsilon_{A}\left(\gamma_{2}, \ldots, \gamma_{s} \mid\left(0:_{M_{1}} \gamma_{2}\right)\right) \\
& =\ldots, \ldots, \\
& =e_{A}\left(. \mid M_{s}\right) \\
& =L_{A}\left(M / \gamma_{1} M+\ldots+\gamma_{s} M\right) .
\end{aligned}
$$

Portanto

$$
e_{A}\left(\gamma_{1}, \ldots, \gamma_{s} \mid M\right)=L_{A}\left(M / \gamma_{1} M+\ldots+\gamma_{s} M\right),
$$

como queríamos demonstrar.

Naturalmente, gostaríamos de obter uma recíproca para o Teorema 2.4.11, mesmo que seja parcial. Para isto, precisamos inicialmente, de um resultado da Álgebra Comutativa. O resultado abaixo, ć uma consequiencia do Teorema da Interseção de kirull [13], pag.293.

Teorema 2.4.12: ([13],pag. 295) Scjam $M$ um $A$-módulo Noctheriano e I um ideal contido no radical de Jacobson de. A. Então

$$
\cap_{n=1}^{\infty} I^{n} M=0
$$

O proximo resultado, fornece uma importante recíproca parcial do Teorema 2.4.11.

Teorema 2.4.13: Sejam $M$ um A-módulo Noetheriano c $\gamma_{1}, \ldots, \gamma_{s}$ um sistema de multiplicidade em $M$, cujos elementos estäo contidos no radical de Jacobson de $A$. Então as seguintes afirmaçōes são equivalentes:

a) $e_{A}\left(\gamma_{1}, \ldots, \gamma_{s} \mid M\right)=L_{A}\left(M / \gamma_{1} M+\ldots+\gamma_{s} M\right)$

b) $\left(\left(\gamma_{1} M+\ldots+\gamma_{i} M\right):_{M} \gamma_{i+1}\right)=\left(\gamma_{1} M+\ldots+\gamma_{i} M\right)$, para $0 \leq i \leq s-1$.

Demonstração: Pelo Teorema 2.4.11, $(b) \rightarrow(a)$. Entrão precisamos apenas provar que $(a) \longrightarrow(b)$.

A prova é por indução sobre $s$. Se $s=1$ temos pela Definição 2.3.1, que

$$
\epsilon_{A}\left(\gamma_{1} \mid M\right)-L_{A}\left(M / \gamma_{1} M\right)-L_{A}\left(0:_{M} \gamma_{1}\right),
$$


e assim desde que $(a)$ é verdadeiro, devemos ter $L_{A}\left(0:_{M} \gamma_{1}\right)=0$. Logo $\left(0:_{M} \gamma_{1}\right)=0$, c isto ó turlo que precisávamos provar neste casso.

De agora cm diante vamos assumir que s $>1$ e também que a afirmação "(a) implica (b)," foi provada para sistrmas de multiplicidades tendo apenas s-1 elementos.

Seja $n_{1}, \ldots, n_{s}$ intciros positivos. Então pelos Teorena 2.4.4, Proposição 2.2 .30 Corolário 2.4.7 segue que

$$
\begin{aligned}
e_{A}\left(\gamma_{1}^{n_{1}}, \ldots, \gamma_{s}^{n_{s}} \mid M\right) & \leq L_{A}\left(M /\left(\gamma_{1}^{n_{1}} M+\ldots+\gamma_{s}^{n_{s}} M\right)\right) \\
& \leq n_{1} \cdots n_{s} L_{A}\left(M /\left(\gamma_{1} M+\ldots+\gamma_{s} M\right)\right) \\
& =n_{1} \cdots n_{s} \epsilon_{A}\left(\gamma_{1}, \ldots, \gamma_{s} \mid M\right) \\
& =c_{A}\left(\gamma_{1}^{n_{1}}, \ldots, \gamma_{s}^{n_{s}} \mid M\right) .
\end{aligned}
$$

Assim temos que

$$
e_{A}\left(\gamma_{1}^{n_{1}}, \ldots, \gamma_{s}^{n_{s}} \mid M\right)=L_{A}\left(M /\left(\gamma_{1}^{n_{1}} M+\ldots+\gamma_{s}^{n_{s}} M\right)\right),
$$

para quaisquer inteiros positivos $n_{1}, \ldots, n_{s}$.

Seja $K=M /\left(0: M \gamma_{1}\right)$. Então segue da sequência exata abaixo e do Teorema 2.3 .7

$$
0 \longrightarrow\left(0: M \gamma_{1}\right) \longrightarrow M \longrightarrow K \longrightarrow 0
$$

a seguinte igualdade numérica

$$
e_{A}\left(\gamma_{1}^{n_{1}}, \ldots, \gamma_{s}^{n_{s}} \mid M\right)=e_{A}\left(\gamma_{1}^{n_{1}} \ldots, \gamma_{s}^{n_{s}} ; K^{\prime}\right)+e_{A}\left(\gamma_{1}^{n_{1}}, \ldots, \gamma_{s}^{n_{s}} \mid\left(0: M \gamma_{1}\right)\right)
$$

Mas pela Proposiçäo 2.3 .19 , desde que $\gamma_{1}^{h_{1}}\left(0:{ }_{M} \gamma_{1}\right)=0$, temos que

$$
e_{A}\left(\gamma_{1}^{n_{1}}, \ldots, \gamma_{S}^{n_{S}} \dot{\gamma}\left(0:_{M} \gamma_{1}\right)\right)=0
$$

e portanto, vale a igualdade

$$
\epsilon_{A}\left(\gamma_{\mathrm{i}}^{n_{1}}, \ldots, \gamma_{s}^{n_{s}} \mid M\right)=\epsilon_{A}\left(\gamma_{1}^{n_{1}} \ldots, \gamma_{s}^{n_{s}} \mid K\right)
$$

Assim por 2.17 e pelo Teorema 2.4.1,

$$
\begin{aligned}
L_{A}\left(M /\left(\gamma_{1}^{n_{1}} M+\ldots+\gamma_{s}^{n_{*}} M\right)\right) & =e_{A}\left(\gamma_{1}^{n_{1}}, \ldots, \gamma_{s}^{n_{s}} \mid K\right) \\
& \leq L_{A}\left(K /\left(\gamma_{1}^{n} K+\ldots+\gamma_{s}^{n_{s}} K\right)\right) \\
& \leq L_{A}\left(M /\left(\left(0:_{M} \gamma_{1}\right)+\gamma_{1}^{n_{1}} K+\ldots+\gamma_{s}^{n_{s}} K\right)\right) .
\end{aligned}
$$


$\Lambda$ íltima desigualdade se justifica pelo Trorema 2.3.13, pois os seguintes módulos

$$
K /\left(\gamma_{1}^{n_{1}} K+\ldots+\gamma_{s}^{n_{s}} K\right): M /\left(\left(0:_{M} \gamma_{1}\right)+\gamma_{1}^{n_{1}} M+\ldots+\gamma_{s}^{n_{s}} M\right)
$$

são isomorfos. Mas desde que

$$
\left(\gamma_{1}^{n_{1}} M \cdots+\gamma_{s}^{n_{s}} M\right) \subseteq\left(\left(0:_{M} \gamma_{1}\right)+\gamma_{1}^{n_{1}} M+\ldots+\gamma_{s}^{n_{s}} M\right)
$$

então

$$
\begin{aligned}
& L_{A}\left(M /\left(\gamma_{1}^{n_{1}} M+\ldots+\gamma_{s}^{n_{s}} M\right)\right)=L_{A}\left(M /\left(\left(0:_{M} \gamma_{1}\right)+\gamma_{1}^{n_{1}} M+\ldots+\gamma_{s}^{n_{s}} M\right)\right) \\
& +L_{A}\left(\left(\left(0::_{M} \gamma_{1}\right)\right.\right. \\
& \left.\left.\left.+\gamma_{1}^{n_{1}} M+\ldots+\gamma_{s}^{n_{s}} M\right) / \gamma_{1}^{n:} M+\ldots+\gamma_{s}^{n_{s}} M\right)\right) \\
& \geq L_{A}\left(M /\left(\left(0:_{M} \gamma_{1}\right)+\gamma_{1}^{n_{1}} M+\ldots+\gamma_{s}^{n_{s}} M\right)\right) \text {. }
\end{aligned}
$$

Portanto

$$
L_{A}\left(M /\left(\gamma_{1}^{n_{1}} M+\ldots+\gamma_{s}^{n_{s}} M\right)\right)=L_{A}\left(M /\left(\left(0:_{M} \gamma_{1}\right)+\gamma_{1}^{n_{1}} M+\ldots+\gamma_{s}^{n_{s}} M\right)\right),
$$

e deste que

$$
\left.\gamma_{1}^{n_{1}} M+\ldots+\gamma_{s}^{n_{s}} M\right) \subseteq\left(0:_{M} \gamma_{1}\right)+\gamma_{1}^{n_{1}} M+\ldots+\gamma_{s}^{n_{s}} M
$$

segue que $\gamma_{1}^{n_{1}} M+\ldots+\gamma_{s}^{n_{s}} M=\left(0:_{M} \gamma_{1}\right)+\gamma_{1}^{n_{1}} M+\ldots+\gamma_{s}^{n_{s}} M$. Logo

$$
\left.\left(0:_{M} \gamma_{1}\right) \subseteq \gamma_{1}^{n_{1}} M+\ldots+\gamma_{s}^{n_{s}} M\right)
$$

para quaisquer inteiros positivos $n_{1}, \ldots, n_{s}$.

Seja $I=\gamma_{1} A+\ldots+\gamma_{s} A$. Então para cada inteiro positivo $n$ temos

$$
\left.\left(0:_{M} \gamma_{1}\right) \subseteq \gamma_{1}^{n_{1}} M+\ldots+\gamma_{s}^{n_{s}} M\right) \subseteq I^{n} M
$$

pois $\gamma_{1}^{n_{1}} \ldots \ldots \gamma_{s}^{n_{s}}$. Porém pclo Teorema 2.1.12,

$$
\cap_{n-1}^{\infty} I^{n} M=0
$$

pois $I$ é um ideal contido no radical de Jacobson de $\Lambda$. Assim temos que $\left(0:_{M} \gamma_{1}\right)=0$.

Scja $M=M / \gamma_{1} M$. Desde que $\left(0: M \gamma_{1}\right)=0$, temos o seguinte isomorfismo de A-rródulos

$$
M /\left(\gamma_{1} M+\ldots+\gamma_{s} M\right) \cong \bar{M} /\left(\gamma_{2} M+\ldots+\gamma_{s} \bar{M}\right)
$$


e segue deste isomortismo que

$$
\begin{aligned}
e_{A}\left(\gamma_{2}, \ldots, \gamma_{s} \mid \bar{M}\right) & -e_{A}\left(\gamma_{1}, \ldots, \gamma_{s} \mid M\right) \\
& =L_{A}\left(M /\left(\gamma_{1} M+\ldots+\gamma_{s} M\right)\right) \\
& =L_{A}\left(\bar{M} /\left(\gamma_{1} \bar{M}+\ldots+\gamma_{s} \bar{M}\right)\right) .
\end{aligned}
$$

Agora ó possível aplicar a hipótese de indutiva. Isto mostra que

$$
\left(\left(\gamma_{2} \bar{M}+\ldots+\gamma_{i} \bar{M}\right):_{\bar{M}} \gamma_{i+1}\right)-\gamma_{2} \bar{M}+\ldots+\gamma_{i} M
$$

para $1 \leq i<s$. Mas

$$
\gamma_{2} M+\ldots+\gamma_{i} M=\left(\gamma_{1} M+\ldots+\gamma_{i} M\right) / \gamma_{1} M
$$

Portanto, pela Proposiçào 2.2 .6 (iii), tenos que

$$
\left(\left(\gamma_{1} M+\ldots+\gamma_{i} M\right):_{M} \gamma_{i+1}\right)=\left(\gamma_{1} M+\ldots+\gamma_{i} M\right),
$$

não apenas para $i=0$, mas também para $1 \leq i<s$.

Teorema 2.4.14: Sejam $M$ um $A$-módulo e $I$ um ideal parâmetro de $A$. Entrào $M$ ć um A-módulo Cohen-Macaulay se, c somente se,

$$
L(M / I M)=e(I, M)
$$

Demonstração: Suponha que $M$ seja um A-módulo Cohen-Macaulay o $I$ um ideal parâmetro de $A$. Se $\operatorname{dim} M=s$, então $I=\left(\gamma_{1}, \ldots, \gamma_{s}\right)$ onde $\gamma_{1}, \ldots, \gamma_{s}$ ć um sistema de parâmetros de $M$, e portanto, $L(M / I M)$ é finito, e além disso, $\gamma_{1}, \ldots, \gamma_{s}$ é urna $M$ sequencia cm $M$ pela condição (b) do Teorema 2.4.13. Portanto $L(M / I M)=e(I ; M)$.

Reciprocamente, suponha que $I$ seja um ideal parâmetro de $A$ e $L(M / I M)=$ e $(I ; M)$. Então pelo Teorema 1.2 .3 temos

$$
\left.\left(\gamma_{1} M+\ldots+\gamma_{i} M\right):_{M} \gamma_{i+1}\right)=\left(\gamma_{1} M+\ldots+\gamma_{i} M\right)
$$

para $0 \leq i \leq s-1$. Mas isto significa que $\gamma_{1}, \ldots, \gamma$ s uma $M$-sequencia em $M$, e portanto, $M$ é um $A$-mósdulo Cohen-. Vacaulay. 


\subsection{A Fórmula Limite de Lech}

Nesta seçâo, vamos expressar a multiplicidade de um ideal $I$ gerado por um sistema de multiplicidade $\gamma_{1}, \ldots, \gamma_{s}$, de uma forma aparentemente mais concreta, isto é, explicitamente dependendo do comprimento do módulo $M /\left(\gamma_{1} M+\ldots+\gamma_{s} M\right)$.

Considere o caso em que $M$ é um $A$-módulo Noetheriano e $\gamma$ seja um sistema de multiplicidade em $M$. Então. desde que

$$
\left(0:_{M} \gamma_{1}\right) \subseteq\left(0:_{M} \gamma_{1}^{2}\right) \subseteq\left(0:_{M} \gamma_{1}^{3}\right) \subseteq \cdots
$$

ć uma cadeia ascendente de submódulos de $M$, existe um inteiro $n>0$ tal que

$$
\left(0: M \gamma_{1}^{n}\right) \subseteq\left(0:_{M} \gamma_{1}^{m}\right) \text { para todo } n \geq m
$$

Assim quando $n \geq m$, temos pela Definição 2.3.1,

$$
\begin{aligned}
e_{A}\left(\gamma^{n} \mid M\right) & =L_{A}\left(M / \gamma^{n} M\right)-L_{A}\left(\left(0:_{M} \gamma_{1}^{n}\right)\right) \\
& =L_{A}\left(M / \gamma^{n} M\right)-L_{A}\left(\left(0:_{M} \gamma_{1}^{m}\right)\right)
\end{aligned}
$$

mas pelo Corolário 2.4.7 do Teorema 2.4.6,

$$
e_{A}\left(\gamma^{n} \mid M\right)-n \epsilon_{A}(\gamma \mid M)
$$

Segue que

$$
L_{\lambda}\left(M / \gamma^{n} M\right)=n e_{A}(\gamma \mid M)+C,
$$

para todo $n \geq m$, onde $C-L_{A}\left(\left(0:_{M} \gamma_{1}^{m}\right)\right)$ é independente de $n$. Fim particular, vemos que

$$
\lim _{n-\rightarrow \infty} \frac{\left(L_{A}\left(M / \gamma^{n} M\right)\right)}{n}=\epsilon_{A}(\gamma \mid M)
$$

Fste ó o caso mais simples da Fórmula Limite de Lech. O resultado mais geral está contido no próximo teorema.

Teorema 2.5.1: (A Fórmula Limite de Lech) Scjam $M$ um A-módulo Noctheriano e $\gamma_{1}, \ldots, \gamma_{s}$ um sistema de multiplicidade em $M$. Então

$$
\lim _{n_{i} \rightarrow \infty} \frac{L_{A}\left(M /\left(\gamma_{1}^{n_{1}} M+\ldots+\gamma_{s}^{n_{s}} M\right)\right)}{n_{1} \cdots n_{s}}=e_{A}\left(\gamma_{1}, \ldots, \gamma_{s} \mid M\right)
$$

onde $n_{1}, \ldots, n_{s}$ são inteiros não-negativos e $n_{i}=\min \left\{n_{1}, \ldots, n_{s}\right\}$. 
Demonstração: Usaremos indução $\mathrm{cm} s$ e obscrvemos que o caso $s=1$ já foi provado acima. Vamos supor que $s>1$ e que a fórmula já foi estabclecida para o caso de sistemas de multiplicidades em $M$, tendo apenas $s-1$ elementos. O objetivo inicial da primeira parté mostrar, que sem perda de generalidades, podemos supor a hipótese adicional de que $\left(0:_{M} \gamma_{1}\right)$ ó o submódulo nulo de $M$. Uma ve\% que isto tenha sido feito, a conclusão decorre facilmente.

Tome $F=M /\left(0:_{M} \gamma_{1}^{p}\right)$, onde $p$ é escolhido suficientemente grande para assegurar que $\left(0: r: \gamma_{1}\right)=0$. Isto pode ser feito pelo Lema 2.4 .3

Segue da sequencia exala abaixo o do Teorema 2.3 .7

$$
0 \longrightarrow\left(0: M \gamma_{1}^{p}\right) \longrightarrow M \longrightarrow F \longrightarrow 0
$$

que

$$
e_{A}\left(\gamma_{1}, \ldots, \gamma_{s} \mid M\right)=e_{A}\left(\gamma_{1}, \ldots, \gamma_{s} \mid F\right)+e_{A}\left(\gamma_{1}, \ldots, \gamma_{s} \mid\left(0:_{M} \gamma_{1}^{p}\right)\right)
$$

Mas pela Proposição 2.3.19, como $\gamma_{1}^{p}\left(0:_{M} \gamma_{1}^{3 p}\right)=0$, temos que

$$
e_{A}\left(\gamma_{1}, \ldots, \gamma_{s} \mid\left(0: M \gamma_{1}^{p}\right)\right)=0
$$

$\log 0$

$$
e_{A}\left(\gamma_{1}, \ldots, \gamma_{s} \mid M\right)=e_{A}\left(\gamma_{1}, \ldots, \gamma_{s} \mid F\right)
$$

Lim seguida do isomorfismo

$$
F /\left(\gamma_{1}^{n_{1}} F+\ldots+\gamma_{s}^{n_{s}} F\right) \cong M /\left(\left(0:_{M} \gamma_{1}^{\beta}\right)+\gamma_{1}^{n_{1}} M+\ldots+\gamma_{s}^{n_{s}} M\right)
$$

e. das inclusões

$$
\gamma_{1}^{n_{1}} M+\ldots+\gamma_{s}^{n_{s}} M \subseteq\left(0: M \gamma_{1}^{p}\right)+\gamma_{1}^{n_{1}} M+\ldots+\gamma_{s}^{n_{s}} M \subseteq M
$$

deduzimos que

$$
\begin{aligned}
0 & \leq L_{A}\left(M /\left(\gamma_{1}^{n_{1}} M+\ldots+\gamma_{s}^{n_{s}} M\right)\right)-L_{A}\left(F /\left(\gamma_{1}^{n_{1}} F+\ldots+\gamma_{s}^{n_{s}} F\right)\right) \\
& -L_{A}\left(\left(\left(0:_{M} \gamma_{1}^{p}\right)+\gamma_{1}^{n_{1}} M+\ldots+\gamma_{s}^{n_{s}} M\right) /\left(\gamma_{1}^{n_{1}} M+\ldots+\gamma_{s}^{n_{s}} M\right)\right. \\
& =L_{A}\left(\left(0:_{M} \gamma_{1}^{p}\right) /\left(0:_{M} \gamma_{1}^{p}\right) \cap\left(\gamma_{1}^{n_{1}} M+\ldots+\gamma_{s}^{n_{s}} M\right)\right) .
\end{aligned}
$$


Ainda, pela inclusão

$$
\gamma_{2}^{n_{2}}\left(0:_{M} \gamma_{1}^{p}\right)+\ldots+\gamma_{s}^{n_{s}}\left(0:_{M} \gamma_{1}^{p}\right) \subseteq\left(0:_{M} \gamma_{1}^{p}\right) \cap\left(\gamma_{1}^{n_{1}} M+\ldots+\gamma_{s}^{n_{s}} M\right)
$$

e pela Proposição 2.2 .3 , tem-se que

$$
\begin{aligned}
B & =L_{A}\left(\left(0:_{M} \gamma_{1}^{p}\right) /\left(0:_{M} \gamma_{1}^{p}\right) \cap\left(\gamma_{1}^{n_{1}} M+\ldots+\gamma_{s}^{n_{s}} M\right)\right) \\
& \leq L_{A}\left(\left(0:_{M} \gamma_{1}^{p}\right) /\left(\gamma_{2}^{n_{2}}\left(0:_{M} \gamma_{1}^{p}\right)+\ldots+\gamma_{s}^{n_{s}}\left(0:_{M} \gamma_{1}^{p}\right)\right)\right) \\
& =n_{2} \cdots n_{s} L_{A}\left(\left(0:_{M} \gamma_{1}^{p}\right) /\left(\gamma_{2}\left(0:_{M} \gamma_{1}^{p}\right)+\ldots+\gamma_{s}\left(0:_{M} \gamma_{1}^{p}\right)\right)\right)
\end{aligned}
$$

Afirmamos que $L_{A}\left(\left(0:_{M} \gamma_{1}^{p}\right) /\left(\gamma_{2}\left(0:_{M} \gamma_{1}^{p}\right)+\ldots+\gamma_{s}\left(0:_{M} \gamma_{1}^{p}\right)\right)\right.$ é finito. Para isto, é suficiente provarmos que $\gamma_{2}, \ldots, \gamma_{s}$ ć um sistema de multiplicidade em $\left(0:_{M} \gamma_{1}^{p}\right)$. Porém $\gamma_{1}^{p}, \gamma_{2}, \ldots, \gamma_{k}$ é um sistema de multiplicidade em $M$, e assim, tambóm é um sistema de multiplicidade em $\left(0:_{M} \gamma_{1}^{p}\right)$. Além disso, $\gamma_{1}^{p}\left(0:_{M} \gamma_{1}^{p}\right)=0$, o por isso, $\gamma_{2}, \ldots, \gamma_{s}$ é um sistema de multiplicidade em $\left(0:_{M} \gamma_{1}^{p}\right)$.

Portanto, pela definição de sistema de multiplicidade,

$$
C=I_{A}\left(\left(0:_{M} \gamma_{1}^{p}\right) /\left(\gamma_{2}\left(0:_{M} \gamma_{1}^{p}\right)+\ldots+\gamma_{s}\left(0:_{M} \gamma_{1}^{p}\right)\right)\right)
$$

é finito.

Estas observaçoos, mostram que temos as seguintes desigualdades numéricas

$$
0 \leq \frac{L_{A}\left(\gamma_{1}^{n_{1}} M+\ldots+\gamma_{s}^{n_{s}} M\right)-L_{A}\left(\gamma_{1}^{n_{1}} F+\ldots+\gamma_{s}^{n_{s}} F\right)}{n_{1} \cdots n_{s}} \leq \underset{n_{1}}{C}
$$

e pela equação (2.18) é suficiente mostrar que

$$
\lim _{n_{i} \times \infty} \frac{L_{A}\left(F /\left(\gamma_{1}^{n_{1}} F+\ldots+\gamma_{s}^{n_{s}} F\right)\right)}{n_{1} \cdots n_{s}}=e_{A}\left(\gamma_{1}, \ldots, \gamma_{s} \mid F\right),
$$

onde $n_{i}=\min \left\{n_{1}, \ldots, n_{s}\right\}$. Em vista disto, ć possível assumir que $\left(0:_{M} \gamma_{1}\right)=0$ é satisfcito.

Scija $\bar{M}=M / \gamma_{1} M$. Então pelo Corolário 2.4 .9 do Teorena 2.4.6, o da Proposição 2.2 .3

$$
\begin{aligned}
0 & \leq n_{1} \cdots n_{s} C_{A}\left(\gamma_{1}, \ldots, \gamma_{s}{ }^{\prime} M\right) \\
& \leq L_{A}\left(M /\left(\gamma_{1}^{n_{1}} M+\ldots+\gamma_{s}^{n_{s}} M\right)\right) \\
& \leq n_{1} L_{A}\left(M /\left(\gamma_{1} M+\gamma_{2}^{n_{2}} M \ldots+\gamma_{s}^{n_{s}} M\right)\right) \\
& =n_{1} L_{A}\left(\bar{M} /\left(\gamma_{2}^{n_{2}} \bar{M}+\ldots+\gamma_{s}^{n_{s}} M\right)\right),
\end{aligned}
$$


pois temos o seguinte isomorfismo

$$
M /\left(\gamma_{1} M+\gamma_{2}^{n_{2}} M \ldots+\gamma_{s}^{n_{s}} M\right) \simeq \bar{M} /\left(\gamma_{2}^{n_{2}} \bar{M}+\ldots+\gamma_{s}^{n_{s}} \bar{M}\right)
$$

Assim sondo temos

$$
\begin{aligned}
0 \leq \epsilon_{A}\left(\gamma_{1}, \ldots, \gamma_{s} \mid M\right) & \leq \frac{L_{A}\left(M /\left(\gamma_{1}^{n_{1}} M+\ldots+\gamma_{s}^{n_{s}} M\right)\right)}{n_{1} \cdots n_{s}} \\
& \leq \frac{L_{A}\left(\bar{M} /\left(\gamma_{2}^{n_{2}} \bar{M}+\ldots+\gamma_{s}^{n_{s}} \bar{M}\right)\right)}{n_{2} \cdots n_{s}}
\end{aligned}
$$

Porém, nossa hipótese indutiva permite concluir que esta expressão tende a

$$
\epsilon_{A}\left(\gamma_{1} \ldots, \gamma_{s} \mid M\right)
$$

e descle que $\left(0: M \gamma_{1}\right)=0$, temos

$$
e_{A}\left(\gamma_{2}, \ldots, \gamma_{s} \cdot \bar{M}\right)=e_{A}\left(\gamma_{1}, \ldots, \gamma_{s} \mid M\right)
$$

como queríamos demonstrar.

\subsection{Funções de Hilbert}

Sejam $A \mathrm{um}$ and $\mathrm{e}, r_{1}, \ldots, x_{\text {s }}$ um conjunto finito de indeterminadas sobre o anel A. Por toda esta sção, vamos denotar o anel dos polinomios $A\left[x_{1}, \ldots, x_{s}\right], s \geq 0$; por $A[X]$. Os anéis graduados gue nos interessam aqui são os anéis dos polinômios. Vamos considerar o anel $A\left[X^{*}\right]$ graduado, da forma usual, pelos inteiros não-negativo. Assim sendo, se $m \geq 0$ é um inteiro, então un polinomio é homogêneo de grau $m$, se cle pode ser expresso na forma

$$
\sum_{\mu_{1}+\ldots+\mu_{s}=m} r_{\mu_{2} \cdots \mu_{s}} x_{1}^{\mu_{1}} \cdots x_{s}^{\mu_{s}}
$$

onde $r_{\mu_{1} \cdots \mu_{s}} \in A$.

Quando $s=0 . A \mid X]$ c exatamente o and $A$ c a graduacào em $A$ c a trivial, isto é, todos os clementos não-nulos são clementos homogêneos de grau zero. 
Seja $M$ um $A[X]$-módulo graduado. Entăo para cada $n \geq 0$, intciro, os elementos homogêneos de $M$ de grau $n$ formam um subgrupo aditivo, denotado por $M_{n}$, do grupo aditivo $M$. Assim temos a seguinte soma direta de $M$

$$
M=M_{0} \oplus M_{1} \oplus \cdots \oplus M_{n} \oplus \cdots
$$

Além disso, se um elemento de $M_{n}$ ć multiplicado por um polinômio homogêneo $r$ do ancl $A$, de grau $m$, então o clemento resultante pertence a $M_{n+m}$ : por definição de graduação. Agora os polinômios homogêneos de grau zero formam um subancl de $A[X]$, que naturalmente identificamos com $A$. Assim cada $M_{n}$ ć um A-módulo.

Defina a função $H: \mathbb{N} \longrightarrow \mathbb{N}$ pela seguinte expressão numérica

$$
H(n, M)=L_{A}\left(M_{n}\right)
$$

Então, $H(n . M)$ é uma função de $n$, para $n=0,1,2, \ldots$ e seus valores são sempre inteiros positivos ou mesmo $\infty$, caso $M_{n}$ tenha comprimento infinito, para pelo menos um valor de $n$.

Definição 2.6.1: A função $H(n, M)$ da equação (2.19), é chamada a função de Hilbert do $A[X]$-módulo graduado $M$.

Do ponto de vista da tcoria da multiplicidade, não é $I(n, M)$ que nos interessa, mas a função

$$
H^{*}(n, M)=I I(0, M)+I I(1, M)+\ldots+H(n, M)
$$

que é chamada a função iterada de Hilbert de $M$.

Sc $M$ é um $A[X]$-módulo graduado e $N$ é um $A[X]_{\text {-submódulo homogêneo de }}$ $M$. cntão a graduação de $M$ induz uma graduação em $N$. $M / N$ também pode ser considerado como um $A[X]$-módulo graduado de modo nat ural. Essas graduações são tais, que na sequência exata usual

$$
0 \rightarrow N \rightarrow M \rightarrow M / N \rightarrow 0
$$


as aplicaçôes preservam grau, isto significa dizer, que um elemento homogôneo sempre é levado num clemento homogêneo de mesmo grau. Segue que, para cada intciro $n \geq 0$, a sequência exatia 2.20 fornece a seguinte sequencia

$$
0 \rightarrow N_{n} \rightarrow M_{n} \rightarrow(M / N)_{n} \rightarrow 0
$$

que tanbém é uma sequencia oxala de A-módulos. Assim sendo, pela aditividade da função comprimento

$$
L_{A}\left(M_{n}\right)-L_{A}\left(N_{n}\right)+L_{A}\left((M / N)_{n}\right),
$$

e pelas equações 2.19 e 2.20 , temos

$$
H(n, M)=H(n, N)+H(n, M / N) .
$$

Mais geralmente, sc

$$
0 \rightarrow N \rightarrow M \rightarrow K \cdot>0
$$

ó uma sequência exata de $A[X]$-módulos em que as aplicaçoes preservam grau, tem-so

$$
H(n, M)=H(n, N)+H(n, K),
$$

para todo $n \geq 0$ e portanto

$$
H^{*}(n, M)=H^{*}(n, N)+H^{*}(n, K)
$$

para todo $n \geq 0$

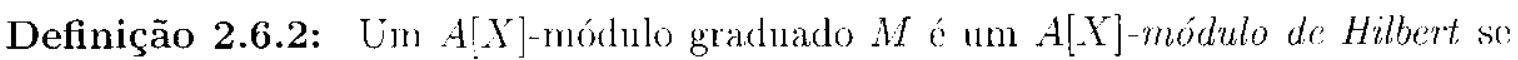
satisfazer as seguintes condiçoes:

i) $M$ é um $A[X]$-módulo finitamente gerado;

ii) $H(n, M)<\infty$ para todo $n \geq 0$.

Exemplo 2.6.3: O ancl dos polinômios $\mathbb{C}[X]$ é um exemplo de um $\mathbb{C}[X]$-módulo de Hilbert, pois $H(n, M)$ é exatamente a dimensào do $\mathbb{C}$-espaço vetorial formado pelos polinomios homogêneos de grau $n$. 
Os próximos resultados: além de sua importância, serão utilizados mais adiante As sulas demonstrações, apesar de não serem extensas, precisam de mais resultados por isso, apcnas ircmos cnunciá-las.

Proposição 2.6.4: ([13], pag. 319) Seja $M$ um $A[X]$-módulo de Hilbert. Então $M$ é um $A[X]$-módulo Noctheriano e $x_{1}, \ldots, x_{s}$ é um sistema de multiplicidade em $M$.

A próxima proposição nos fornece vários exemplos de módulos de Hilbert.

Proposição 2.6.5: ([13], pag. 318) Scjam $M$ um $A[X]$-módulo de Hilbert c $N$ um submódulo homogèneo do $M$. Fntão $N$ e $M / N$ com as graduações usuais também são $A[X]$-módulos de Hilbert.

Proposição 2.6.6: ([13], pag. 319) Scjam $M$ um $A[X]$-módulo de Hilbert e suponha que $s=0$. Então $H(n, M)=0$, para todo $n$ suficicntemente grande. Além disso, $L_{A}(M)$ é finito e $I^{*}(n, M)=L_{A}(M)$, para todo $n$ suficientemente grande.

A Proposição 2.6.6, nos diz tudo sobre a função de Hilbert de: um módulo de Hilbert, no caso onde o número de variáveis é zcro.

Teorema 2.6.7: ([13],pag. 320) Seja $M$ um $A\left[x_{1}, \ldots, x_{s}\right]$-módulu de Hilbert. Então para $n$ suficientemente grande, $H(n, M)$ é dado por uma relação da forma

$$
H(n, M)=\sum_{v=0}^{s-1} c_{v}\left(\begin{array}{c}
n+v \\
v
\end{array}\right)
$$

onde $c_{0}, c_{1}, \ldots, c_{s}$, são inteiros independentes de $n$. Além disso, a expressão (2.23) ćminicil.

Observação 2.6.8: O Teorema 2.6.7 é o resultado chave da teoria das funçós de Ilibert. Primeiro, note que $\left(\begin{array}{c}n+v \\ v\end{array}\right)$ denota o coeficiente binomial usual, isto é,

$$
\left(\begin{array}{c}
n+v \\
v
\end{array}\right)=\frac{(n+v)(n+v-1) \cdots(n+1)}{1.2 \cdots v}
$$


de modo que, para um valor fixo de $v \geq 0,\left(\begin{array}{c}n+v \\ v\end{array}\right)$ ś um polinomio em $n$ de grau v. cujo termo dominante é $\frac{n^{v}}{v !}$. O Teorema 2.6 .7 mostra portanto, que para valores suficientemente grande de $n$,

$$
H(n, M)-c_{s-1} \frac{n^{s-1}}{(s-1) !}+\text { termos de menor gran }
$$

ć um polinomio em $n$, cujo grau é nenor on igual do que $s-1$.

Observação 2.6.9: () Teorema 2.6.7 nos diz que se $M$ é um $A[X]$-módulo de Hilbert, então existem inteiros unicamentc determinados por $M$,

$$
h_{0}(M), h_{1}(M), \ldots, h_{s-1}(M)
$$

tais que

$$
I(n, M)=\sum_{v=0}^{s \cdots 1} h_{v}(M)\left(\begin{array}{c}
n+v \\
v
\end{array}\right)
$$

para todo $n$ suficientemente grande.

Listaremos agora duas propriedades dos coeficientes binomiais que serão utilizarlos no decorrer desta seção.

i) Para quaisquer inteiros $n, v$, com $n \geq 1, v \geq 1$ tem-se:

$$
\left(\begin{array}{c}
n+v \\
v
\end{array}\right)-\left(\begin{array}{c}
n+v-1 \\
v-1
\end{array}\right)=\left(\begin{array}{c}
n-1+v \\
v
\end{array}\right)
$$

ii) Para quaisquer inteiros $n$ e $v$ năo-negativos vale:

$$
\sum_{k=0}^{n}\left(\begin{array}{c}
k+v \\
v
\end{array}\right)=\left(\begin{array}{c}
u+v-1 \\
v+1
\end{array}\right)
$$

Nós iremos chamar os inteiros $h_{\eta}(M), v=0,1, \ldots, s-1$ de os coeficientes de Hilbert de $M$. A expressão (2.24) mostra que

$$
H(k, M)=\sum_{v-0}^{s-1} h_{v}(M)\left(\begin{array}{c}
n+v \\
v
\end{array}\right)+\mu_{k}
$$


para todo $k \geq 0$, onde $\mu_{0}, \mu_{1}, \ldots, \mu_{s}$ é nma sequência de inteiros com a propriedade que $\mu_{k}=0$, desde que $k$ seja suficientemente grande. Suponha quc $\mu_{k}-0$ quando $k>q$. Se agora $k>q$ e somarmos a equação $(2.27)$ para $k-0,1, \ldots, n$, então encontraremos

$$
\begin{aligned}
H^{*}(n, M) & =H(0, M)+H(1, M)+\ldots+H(n, M) \\
& -\sum_{v=0}^{s-1} h_{v}(M) \sum_{k=0}^{n}\left(\begin{array}{c}
k-v \\
v
\end{array}\right)+\sum_{k=0}^{\infty} \mu_{k} \\
& =\sum_{k=0}^{\infty} \mu_{k}+\sum_{v=0}^{s} h_{v}(M)\left(\begin{array}{c}
n+v+1 \\
v+1
\end{array}\right)
\end{aligned}
$$

em virtude da equação (2.26). Segue que existem únicos inteiros

$$
h_{0}^{*}(M), h_{v}^{*}(M), \ldots, h_{s}^{*}(M)
$$

tais que

$$
H^{*}(n, M)=\sum_{v=0}^{s} h_{1}^{*}(M)\left(\begin{array}{c}
n \cdots v \\
v
\end{array}\right),
$$

desde que $n$ seja suficientemente grande. Além disso,

$$
h_{0}(M), h_{1}(M) \ldots, h_{s-1}(M)
$$

são os mesmos que os múmeros

$$
h_{1}^{*}(M), h_{1}^{*}(M), \ldots, h_{s}^{*}(M)
$$

Fin particular, quando $s \geq 1$ temos

$$
h_{s}^{*}(M)=h_{s} \quad 1(M) .
$$

Além disso, prova-se cm ([13]. pag. 324) que

$$
H(n, M)=h_{s}^{*}(M) \frac{n^{s-1}}{(s-1) !}+\cdots
$$

0

$$
h_{s}^{*}(M)=h_{s-1}^{*}\left(M / x_{s} M\right)-h_{s-1}^{*}\left(0:_{M} x_{s}\right)
$$


O próximo teorema, mostra que se $M$ é um $A\left[x_{1}, \ldots, x_{s}\right]$-módulo de Hilbert, então a multiplicidade de um sistema de multiplicidade $\gamma_{1}, \ldots, \gamma_{s}$ é o coeficiente do maior grau do polinônio de Hilbert.

Teorema 2.6.10: Seja $M$ um $A\left[x_{1}, \ldots, x_{s}\right]$-módulo de Hilbert. Então

$$
h_{s}^{*}(M)=e_{A[X]}\left(\gamma_{1}, \ldots, \gamma_{s} \mid M\right)
$$

Demonstração: Usaremos indução sobre s. Se s -0 , entăo $A\left[x_{1}, \ldots, x_{s}\right]=A \mathrm{e}$ daí

$$
e_{A[X]}\left(\gamma_{1}, \ldots, \gamma_{s} \mid M\right)=e_{A}(. \mid M)=L_{A}(M)
$$

Por outro laxto, a Proposição 2.6.6 mostra que $h_{s}^{*}(M)=L_{A}(M)$ neste caso particular.

Vamos supor que $s \geq 1$ e que o teorema já foi provado quando ajenas $s-1$ indeterminadas estão envolvidas. Pela Proposiçäo 2.6.4, $\epsilon_{A[X]}\left(\gamma_{1}, \ldots, \gamma_{s} \mid M\right)$ está bem definido. Agora

$e_{A \mid X]}\left(x_{1}, \ldots, x_{s-1}, x_{s} \mid M\right)=e_{\left.A_{1} X\right\}}\left(x_{1}, \ldots, x_{s-1} \mid M / x_{s} M\right) \cdots e_{\left.A_{l} X\right]}\left(x_{1}, \ldots, x_{s-1} \mid\left(0:_{M} x_{s}\right)\right)$.

Além disso, o anel $A\left[x_{1}, \ldots, x_{s}\right] /\left(x_{s}\right)$ ć isomorfo ao anel $A\left[x_{1}, \ldots, x_{s-1}\right]$ eo isomorfismo 6 tal que, para cada $1 \leq i \leq s-1$, a imagem de $x_{i}$ em $A\left[x_{1}, \ldots, x_{s}\right] /\left(x_{s}\right)$ corresponde a $x_{i}$ considerado como um elemento de $A\left[x_{1}, \ldots, x_{s-1}\right]$. Note que o ideal $\left(x_{s}\right)$ anula $M / x_{s} M$. Então podemos aplicar o fato de que

$$
e_{A\left[x_{1}, \ldots, x_{s-1}\right]}\left(x_{1}, \ldots, x_{s-1} \mid M / x_{s} M\right)=e_{A\left[x_{1}, \ldots, x_{s-1} \mid\right.}\left(x_{1}, \ldots, x_{s} \mid M / x_{s} M\right)
$$

Mas pelo Proposição $2.6 .5, M / x_{s} M$ é um $A\left[x_{1}, \ldots x_{s-1}\right]$-módulo de Hilbert. Assim sendo, pela hipótese indutiva

$$
e_{\left.A x_{1}, \ldots, x_{s-1}\right]}\left(x_{1}, \ldots, x_{s-1} \mid M / x_{s} M\right)-h_{s-1}^{*}\left(M / x_{s} M\right) .
$$

Analogamente podemos mostrar que

$$
e_{A} i_{\left.1, \ldots, r_{s-1}\right]}\left(x_{1}, \ldots, x_{s-1} \mid\left(0:_{M} x_{s}\right)\right)=h_{s-1}^{*}\left(\left(0:_{M} x_{s}\right)\right)
$$

Assim

$$
\begin{aligned}
e_{A}\left[X_{-}\left(x_{1}, \ldots, x_{s} \mid M\right)\right. & =h_{s-1}^{*}\left(M / x_{s} M\right) \cdots h_{s-1}^{*}\left(0:_{M} x_{s}\right) \\
& =h_{s}^{*}(M)
\end{aligned}
$$

pela cquagão (2.30). 


\subsection{A Fórmula Limite de Samuel}

Seja $M$ um $A$-módulo Noetheriano e $\gamma_{1} \ldots \gamma_{s}$ um sistema de multiplicidade em $M$. Considere o ideal $I$ do anel $A$ gerado por este sistema de multiplicidade, isto é,

$$
I=\gamma_{1} A+\ldots+\gamma_{s} A \text {. }
$$

Temos que $I$ é um ideal de $A$ finitamente gerado e usarcmos os símbolos $x_{1}, \ldots, x_{s}$, para denotar as indeterminadas sobre o anel $A$. Note que existe exatamente uma indeterminada para cada elemento do sistema de multiplicidade.

Sejam $n, n_{1}, \ldots, n_{s}$ números inteiros positivos. Então temos as scguintes inclusões

$$
\gamma_{1}^{n_{1}} M+\ldots+\gamma_{s}^{n_{s}} M \subseteq I^{n} M \subseteq M
$$

Daí temos

$$
L_{A}\left(M /\left(\gamma_{1}^{n_{1}} M+\ldots+\gamma_{s}^{n_{s}} M\right)\right)=L_{A}\left(M / I^{n} M\right)+L_{\Lambda}\left(A^{n} M /\left(\gamma_{1}^{n_{1}} M+\ldots+\gamma_{s}^{n_{s}} M\right)\right) .
$$

Como $\gamma_{1}^{n_{1}}, \ldots, \gamma_{s}^{n_{s}}$ ó um sistema de multiplicidade em $M$ então $L_{A}\left(M /\left(\gamma_{1}^{n_{1}} M+\right.\right.$ $\left.\left.\ldots+\gamma_{s}^{n_{s}} M\right)\right)$ é finito. Segue que $L_{A}\left(M / I^{n} M\right)$ é finito para todo inteiro positivo $n$.

Seja

$$
G_{I}(M)=(M / I M) \oplus\left(I M / I^{2} M\right) \oplus \cdots .
$$

$\Lambda$ firmamos que $G_{I}(M)$ admite uma estrutura de $A[X]$-módulo, no qual o grupo dos elementos homogêneos de grau $n$ são precisamente $I^{n} M / I^{n+1} M$, para qualquer intciro $n \geq 0$. Para isto, seja $\eta$ um elemento de $I^{n} M / I^{n+1} M$, isto é,

$$
\eta=y+I^{n+1} M
$$

onde $y \in I^{n} M$

Seja

$$
\phi\left(x_{1}, \ldots, x_{s}\right)=\sum_{\mu_{1}+\ldots+\mu_{s}-m} r_{\mu_{1} \cdots \mu_{s}} x_{1}^{\mu_{1}} \cdots x_{s}^{\mu_{s}}:
$$

um elemento homogêneo de $A[X]$ de grau $m$, onde $r_{\mu_{1} \cdots \mu_{s}} \in A$. Então $\phi\left(x_{1}, \ldots, x_{s}\right) \eta$ ć un elemento de $I^{n+m} M / I^{n+m+1} M$ que é representado por

$$
\sum_{\mu_{1}+\ldots+\mu_{s}-m_{n}} r_{\mu_{1} \ldots \mu_{s}} \gamma_{1}^{\mu_{1}} \cdots \gamma_{s}^{\mu_{s}} y
$$


Verifica-se, que com este produto definido acima, $G_{I}(M)$ torna-se um $A[X]$-módulo graduado.

Desde que $M$ é um A-módulo Noctheriano, ele é finitamente gerado. Seja $m_{1} \ldots, m_{q}$, um conjunto gerador de $M$, então

$$
M-R m_{1}+\ldots+R m_{\eta}
$$

e denote por $\tilde{m}_{i}$ a imagem de $m_{i}$ em $M / I M$ de modo que $\tilde{m}_{i}$ seja um elemento homogeneo de $C_{l}(M)$ de graun zero. Fntão

$$
G_{I}(M)=A[X] \tilde{m}_{1}+\ldots+A[X] \tilde{n}_{q}
$$

Agora,

$$
L_{\Lambda}\left(M_{n}\right)=L_{A}\left(I^{n} M / I^{n+1} M\right)
$$

é finito para todo $n$. Assim sendo $G_{I}(M)$ é um $A[\mathrm{X}]$-módulo de Hilbert. Além disso, pola equaçào (2.31),

$$
L_{A}\left(M / I^{n} M\right)=L_{A}\left(M_{0}\right)+L_{A}\left(M_{1}\right)+\ldots+L_{A}\left(M_{n} 1\right),
$$

pois para quaisquer $n \geq 0$. De fato das seguintes inclusões

$$
I^{n+1} M \subset I^{n} M \subseteq M
$$

obtemos as igualdades numéricas

$$
\begin{aligned}
L\left(M_{0}\right) & =L(M / I M) \\
L\left(M_{1}\right)=L\left(I M / I^{2} M\right) & =L\left(M / I^{2} M\right)-L(M / I M) \\
L\left(M_{2}\right)=L\left(I^{2} M / I^{3} M\right) & -L\left(M / I^{3} M\right)-L\left(M / I^{2} M\right) \\
\cdots & =\cdots \\
L\left(M_{n-1}\right)=L\left(I^{n}{ }^{1} M / I^{n} M\right) & =L\left(M / I^{n} M\right)-L\left(M / I^{n-1} M\right) .
\end{aligned}
$$

Mas, somando todas estas igualdades, obtemos a equação (2.32), ou seja,

$$
L_{\lambda}\left(M / I^{n} M\right)=H^{*}\left(n-1, G_{I}(M)\right) .
$$


Portanto, pela equação (2.28), para $n$ suficientemente grande, $L_{1}\left(M / I^{n} M\right)$, é um polinômio cujo grau não excede s. De fato, novamente pela equação (2.28), tem-se que

$$
L_{A}\left(M / I^{n} M\right)=h_{s}^{*}(M) \frac{n^{s}}{s !}+\text { termos de menor ordem }
$$

para $n$ suficientemente grande.

Defina $e(s, A, M)$ da seguinte forma

$$
e(s, A, M)=\lim _{n \rightarrow \infty} \frac{L_{A}\left(M / I^{n} M\right)}{n^{s} / s !}=h_{s}^{*}\left(G_{l}(M)\right) .
$$

Para simplificar a notação, vamos denotar o $A$-módulo $G_{l}(M)$, simplesmente por $G(M)$.

Lema 2.7.1: Scja $I$ um idcal de $A\left[x_{1}, \ldots, x_{s}\right]$ gerado pelas indeterninadas $x_{1}, \ldots, x_{s}$ c scja $n$ um intciro satisfazendo $n \geq n_{1}-\ldots+n_{s}$. onde $n_{1}, \ldots, n_{s}$ são inteiros nầnegativos. Então

$L_{A}\left(G(M) /\left(x_{1}^{n_{1}} G(M)+\ldots+x_{s}^{n_{s}} G(M)\right)\right)=L_{A X]}\left(G(M) /\left(x_{1}^{n_{1}} G(M)+\ldots+x_{s}^{n_{s}} G(M)\right)\right)$

Demonstração: De fato, das seguintes inclusões

$$
x_{1}^{n_{1}} G(M)+\ldots+x_{s}^{n_{s}} G(M) \subseteq I^{n} G(M) \subseteq G(M),
$$

temos que

$L_{\Lambda}\left(G(M) /\left(x_{1}^{n_{1}} G(M)+\ldots+x_{s}^{n_{s}} G(M)\right)\right) \geq L_{\Lambda[X]}\left(G(M) /\left(x_{1}^{n_{1}} G(M)+\ldots+x_{s}^{n_{s}} G(M)\right)\right)$

$L_{A}\left(I^{n} G(M) /\left(x_{1}^{n !} G(M)+\ldots+x_{s}^{n_{*}} G(M)\right)\right) \geq L_{A}{ }^{n}\left(I^{n} G(M) /\left(x_{1}^{n_{1}} G(M)+\ldots+x_{s}^{n_{*}} G(M)\right)\right)$, pois todo $A[X]$-submódulo também é um A-submódulo. Assim sendo, o resultado seguirá do seguinte fato

$$
L_{A}\left(G(M) / I^{n} G(M)\right)-L_{A} X !\left(G(M) / I^{n} G(M)\right)<\infty .
$$


Mas

$$
L_{A}\left(G(M) / I G_{A}(M)\right)=\sum_{v=0}^{n-1} L_{A}\left(I^{v} G(M) / I^{v+1} G(M)\right)
$$

$$
L_{A X X]}\left(G(M) / I^{n} G(M)\right)=\sum_{n=-1}^{n+1} L_{A\{X ?}\left(I^{n} M / I^{n+1} G(M)\right)
$$

e cada elemento $x_{1}, \ldots, x_{s}$ anula $I^{\prime \prime} G(M) / I^{v+1} G(M)$.

Assim os $A[X]$-submódulos $I^{v} G(M) / I^{v+1} G(M)$ coincidem quando vistos como A-submódulos, e assim temos

$$
L_{A}\left(G(M) / I^{n} G(M)\right)-L_{A X]}\left(G(M) / I^{n} G(M) .\right.
$$

Mas pela Proposição 2.6.4, $x_{1}, \ldots, x_{x}$ é um sistema de multiplicidade em $G(M)$ e daí

$$
L_{A 1}\left(G(M) / I^{n} G(M)\right)<L_{A}\left(M /\left(x_{1}^{n_{1}} M+\ldots+x_{s}^{n_{s}} M\right)\right)<\infty
$$

c assim $L_{A[X]}\left(G(M) / I^{n} G(M)\right)$ é finito.

Finalmente

$$
\begin{aligned}
L_{A\left[Y_{i}\right.}\left(G(M) / I^{n} G(M)\right) & =L_{A X_{0}^{\prime}}\left(G(M) /\left(x_{1}^{n_{1}} G(M)+\ldots+x_{s}^{n_{s}} G(M)\right)\right) \\
& +L_{A i X_{j}}\left(I^{n} G(M) /\left(x_{1}^{n_{1}} G(M)+\ldots+x_{s}^{n_{s}} G(M)\right)\right) \\
& \leq L_{\Lambda}\left(G(M) /\left(x_{1}^{n_{1}} G(M)+\ldots+x_{s}^{n_{s}} G(M)\right)\right) \\
& +L_{A}\left(I^{n} G(M) /\left(x_{1}^{n_{1}} G(M)+\ldots+x_{s}^{n_{s}} G(M)\right)\right) \\
& =L_{A}\left(G(M) / I^{n} G(M)\right) .
\end{aligned}
$$

c isto implica que

$$
L_{A}\left(G(M) /\left(x_{1}^{u_{1}} G(M)+\ldots+x_{s}^{n_{s}} G(M)\right)\right)=L_{A[X} X_{j}\left(G(M) /\left(x_{1}^{u_{1}} G(M)+\ldots+x_{s}^{n_{s}} G(M)\right)\right)
$$

como queríamos demonstrar.

Teorema 2.7.2: (Fórmula Limite de Samuel) Scja $M$ um A-módulo Noetheriano e $\gamma_{1}, \ldots \gamma$ um sistema de multiplicidade em $M$. Se agora $I=\gamma_{1} A+\ldots+\gamma_{s} A$, entăo

$$
\lim _{n \rightarrow \infty} \frac{L_{A}\left(M / I^{n} M\right)}{n^{s} / s !}=\epsilon_{A}\left(\gamma_{1}, \ldots, \gamma_{s} \mid M\right)
$$


Além disso,

$$
e_{A}\left(\gamma_{1}, \ldots, \gamma_{s} \mid M\right)=e_{A[X]}\left(\gamma_{1}, \ldots, \gamma_{s} \mid G(M)\right)
$$

onde

$$
G(M)=(M / I M) \oplus\left(I M / I^{2} M\right) \oplus \cdots
$$

Demonstração: A demonstração é por indução sobre s. So $s=0$ obtemos pela rquação (2.34).

$$
e(0,(0), M)=\lim _{n \rightarrow \infty} \frac{M /(0) M}{n^{0} 0 !}-L_{A}(M) .
$$

Agora suponha que $s \geq 1$ o ponha $\bar{M}=M / \gamma_{1} M, I=\gamma_{2} A+\ldots+\gamma_{s} A$. Então $\bar{M}$ é um A-modulo Noetheriano e admite $\gamma_{2} \ldots, \gamma_{s}$ como um sistema de multiplicidade. Como $\gamma_{1}$ anula $M$, temos $(I)^{n} M=I^{n} \bar{M}$ e portanto temos

$$
\bar{M} /(\bar{I})^{n} \bar{M} \cong M /\left(\gamma_{1} M+I^{n} M\right)
$$

Assim sendo

$$
\begin{aligned}
L_{A}\left(M /(I)^{n} M\right) & =L_{A}\left(M / I^{n} M\right)-L_{A}\left(\left(\gamma_{1} M+I^{n} M\right) / I^{n} M\right) \\
& =L_{A}\left(M / I^{n} M\right)-L_{A}\left(\gamma_{1} M /\left(\gamma_{1} M \cap I^{n} M\right)\right) \\
& =L_{A}\left(M / I^{n} M\right)-L_{A}\left(\gamma_{1} M / \gamma_{1}\left(I^{n} M:_{M} \gamma_{1}\right)\right),
\end{aligned}
$$

pela Proposição 2.2.6 (ii).

Porém o epimorfismo

$$
M \longrightarrow \gamma_{1} M
$$

produzido pela multiplicação por $\gamma_{1}$, ́ tal que a imagem inversa de $\gamma_{1}\left(A^{n} M:_{M} \gamma_{1}\right)$ é exatamente $\left(A^{n} M:_{M} \gamma_{1}\right)$. Assim temos pelo Teorema 2.3 .13 o isomorfismo

$$
\gamma_{1} M / \gamma_{1}\left(I^{n} M:_{M} \gamma_{1}\right) \cong M /\left(I^{n} M:_{M} \gamma_{1}\right),
$$

e assim

$$
\begin{aligned}
L_{A}\left(\bar{M} /(\bar{I})^{n} \bar{M}\right) & =L_{A}\left(M / I^{n} M\right)-L_{A}\left(M /\left(I^{n} M:_{M} \gamma_{1}\right)\right. \\
& \geq L_{A}\left(M / I^{n} M\right)-L_{A}\left(M / I^{n}{ }^{1} M\right),
\end{aligned}
$$

pois $I^{n-1} M \subseteq\left(I^{n} M:_{M} \gamma_{1}\right)$. Him seguida pelas equações (2.33) e (2.34) temos

$$
L_{A}\left(M / I^{n} M\right)=e(s, I, M) \frac{n^{s}}{s !}+\text { termos de menor ordem, }
$$


para $n$ suficientemente grande. Segue que para $n$ suficientemente grande

$$
L_{A 1}\left(M / I^{n} M\right)-L_{A}\left(M / I^{n-1} M\right)=c(s, I, M) \frac{n^{s-1}}{(s-1) !} \text { t termos de menor ordem, }
$$

c. portanto

$$
L_{A}\left(\bar{M} /(\bar{I})^{n} \bar{M}\right) \geq e(s, I, M) \frac{n^{s} 1}{(s-1) !}+\text { termos de menor ordem. }
$$

Vamos dividir por $\frac{n^{s-1}}{(s-1) !}$ e depois lazer $n$ tender para o infinito. Isto nos fornece

$$
\ell(s-1, \bar{I}, \bar{M}) \geq \ell(s, I, M)
$$

Se $s \geq 2$, então o argumento acima pode ser repetido para obtermos

$$
e(s-2, \dot{\bar{I}}, \overline{\bar{M}}) \geq e(s-1, \bar{I}, \bar{M})
$$

onde $\overline{\bar{I}}-\gamma_{3} A+\ldots+\gamma_{s} A$ e $\overline{\bar{M}}=M / \gamma_{2} \bar{M}$. Mas pela Proposição 2.2 .6 (iii). $\bar{M}$ ć isomorfo a $M /\left(\gamma_{1} M+\gamma_{2} M\right)$ e assim provamos que

$$
\epsilon(s, I, M) \leq e(s-1, I, \bar{M}) \leq e\left(s-2, \bar{I}, M /\left(\gamma_{1} M+\gamma_{2} M\right)\right)
$$

Procedendo desta forma obteremos

$$
e(s, I, M) \leq e\left(0,(0), M / \gamma_{1} M+\ldots+\gamma_{s} M\right)=L_{A}\left(M /\left(\gamma_{1} M+\ldots+\gamma_{s} M\right)\right.
$$

pela equação (2.36), on mudando a notação,

$$
\lim _{n \rightarrow \infty} \frac{L_{A}}{n^{s} / s !} \frac{M / I^{n}}{M)} \leq L_{\Lambda}\left(M / \gamma_{1} M+\ldots+\gamma_{s} M\right) .
$$

Vesta relação podemos substituir $\gamma_{1}, \ldots \gamma_{s}$ por $\gamma_{1}^{p} \ldots \gamma_{s}^{p}$, onde $p$ é urm inteiro positivo arbitrário e observemos ao mesmo tempo que

$$
\left(\gamma_{l}^{p} I+\ldots+\gamma_{s}^{p} I\right)^{n} \subseteq\left(\gamma_{1} I+\ldots+\gamma_{s} I\right)^{n p}
$$

Este artifício mostia clue

$$
\begin{aligned}
\lim _{n \rightarrow \infty} \frac{L_{A}\left(M /\left(\gamma_{1} M+\ldots+\gamma_{s} M\right)^{n p} M\right)}{n^{s} / s !} & \leq \lim _{n \rightarrow \infty} \frac{L_{A}\left(M /\left(\gamma_{1}^{p} M+\ldots+\gamma_{s}^{p} M\right)^{n} M\right)}{n^{s} / s !} \\
& \leq \frac{L_{A}\left(M /\left(\gamma_{1}^{p} M+\ldots+\gamma_{s}^{p} M\right)\right)}{p^{s}}
\end{aligned}
$$


Mas, o primeiro desses limites é e $(s, I, M)$. Portanto

$$
e(s, I, M) \leq \frac{L_{A}\left(M /\left(\gamma_{1}^{p} M+\ldots+\gamma_{s}^{p} M\right)\right)}{p^{s}}
$$

Agora fazendo $p$ tender para o infinito c aplicando a Fórmula Jimite de Lech, isto nos dá

$$
e(s, I, M) \leq e_{A}\left(\gamma_{1}, \ldots, \gamma_{s} \mid M\right) .
$$

Resta estabelecer a desigualdade oposta. Para este fim, seja $n_{1}, \ldots, n_{s}$ inteiros positivos. Ponha

$$
U=\ominus_{n-0}^{\infty}\left\{I^{n} M \Gamma\left(I^{n+1} M+\gamma_{1}^{n_{1}} M+\ldots+\gamma_{s}^{n_{s}} M\right) / I^{n+1} M\right\} .
$$

É fácil verificar que $U$ ó um $A[X]$-submódulo homogêneo de $G(M)$ e que

$$
x_{1}^{n_{1}} G(M)+\ldots+x_{s}^{n_{s}} G(M) \subseteq U
$$

Assim sendo

$$
L_{A}\left(G(M) / x_{1}^{n_{1}} G(M)+\ldots+x_{s}^{n_{s}} G(M)\right) \geq L_{A}(G(M) / U) .
$$

Tome agora $F=\gamma_{1}^{n_{1}} M+\ldots+\gamma_{s}^{n_{s}} M$. Então temos o isomorfismo

$$
G(M) / U \cong \oplus_{n=0}^{\infty}\left\{I^{n} M \cap\left(I^{n+1} M+F\right)\right\}
$$

de $A$-módulos. Mas

$$
\begin{aligned}
I^{n} M /\left(I^{n} M \bigcap\left(I^{n+1} M+F\right)\right) & \cong\left(I^{n} M+I^{n+1} M+F\right) /\left(I^{n+1} M+F\right) \\
& \cong\left(I^{n} M+F\right) /\left(I^{n+1} M+F\right),
\end{aligned}
$$

c assim

$$
G(M) / U \cong \oplus_{n=0}^{\infty}\left(I^{n} M+F\right) /\left(I^{n+1} M+F\right) .
$$

Porćm quando $n \geq n_{1}+\ldots+n_{s}$ temos $I^{n} M \subseteq F$ e portanto $I^{n} M=F$. Segue então que?

$$
L_{A}(G(M) / U)=\sum_{n \geq 0} L_{A}\left(\left(I^{n} M+F\right) /\left(I^{n+1} M+F\right)\right)=L_{A}(M / F),
$$

e então pola equação (2.38) que

$$
L_{A}\left(M /\left(\gamma_{1}^{n_{1}} M+\ldots+\gamma_{s}^{n_{s}} M\right)\right) \leq L_{A[X]}\left(G(M) /\left(a_{1}^{n_{1}} G(M)+\ldots+x_{s}^{n_{s}} G(M)\right)\right)
$$


Mas desde que

$$
\underline{L_{A}\left(M /\left(\gamma_{1}^{n_{1}} M+\ldots+\gamma_{s}^{n_{s}} M\right)\right)} \frac{\left.L_{A|X|} G(M) /\left(x_{1}^{n_{1}} G(M)+\ldots+x_{s}^{n_{s}} G(M)\right)\right)}{n_{1} \cdots n_{s}},
$$

segue pela Fórmula Limite de Lech que

$$
e_{A}\left(\gamma_{1}, \ldots, \gamma_{s} \mid M\right) \leq e_{A \mid X\}}\left(\gamma_{1}, \ldots, \gamma_{s} \mid G(M)\right)
$$

Mas pelo Teorema 2.6 .10 e prola equação (2.34), que

$$
\epsilon_{A \mid X j}\left(\gamma_{1}, \ldots, \gamma_{s} \mid G(M)\right)=h_{s}^{*}(G(M)) \cdots c(s, I, A) .
$$

Assim $c_{A}\left(\gamma_{1}, \ldots, \gamma_{s} \mid M\right) \leq e(s, I, A)$, que em vista da equação (2.38) completa a demonstração do teorema.

Teorema 2.7.3: ([13], pag. 330) Sejam $M$ um $A$-módulo Noetheriano, $\gamma_{1}, \ldots, \gamma_{s}$ e $\gamma_{1}^{\prime}, \ldots, \gamma_{s}^{\prime}$ dois sistemas de multiplicidades em $M$. Suponha que

$$
\gamma_{1} M+\ldots+\gamma_{s} M \subseteq \gamma_{1}^{\prime} M+\ldots+\gamma_{s}^{\prime} M
$$

i) Suponha que

$$
\gamma_{1} M+\ldots+\gamma_{s} M \subseteq \gamma_{1}^{\prime} M+\ldots+\gamma_{s}^{\prime} M
$$

Então para qualquer submódulo $N$ do $M$ tem-se a seguinte desigualdade

$$
e_{A}\left(\gamma_{1}, \ldots, \gamma_{s} \mid N\right) \geq e_{A}\left(\gamma_{1}^{\prime}, \ldots, \gamma_{s}^{\prime} \mid N\right)
$$

A afirmação continua válida se substituirmos $N$ por um módulo cuociente de $M$.

ii) Suponha que

$$
\gamma_{1} M+\ldots+\gamma_{s} M=\gamma_{1}^{\prime} M+\ldots+\gamma_{s}^{\prime} M
$$

Então para qualquer submódulo $N$ de $M$ tem-se a seguinte desigualdade:

$$
e_{A}\left(\gamma_{1}, \ldots, \gamma_{s} \mid N\right)=e_{A}\left(\gamma_{1}^{\prime}, \ldots, \gamma_{s}^{\prime} \mid N\right)
$$

A afirmarăa continua válida se substituirmos $N$ por um módulo quociente de $M$. 


\section{Capítulo 3}

\section{Multiplicidades Mixtas de dois Ideais $\mathcal{M}$-primários}

\subsection{Introdução}

Seja $(A, \mathcal{M})$ um ancl local de dimensào $d$. Seja $I$ um ideal $\mathcal{M}$-primário. Samuel [20] provou que o comprimento do ideal $I^{r}$, ó um polinônio em $r$, para valores suficientemente grande de $r$, cujo grau ś igual a dimensão de A. Além disso, o coeficiente de maior grau deste polinomio é chamado a multiplicidade do ideal $I$, denotada por $e(I, A)$.

Scjam $B$ um anel Artiniano primário e $X=\left\{x_{0}, x_{1}, \ldots, x_{m}\right\}$ e $Y=\left\{y_{0}, y_{1}, \ldots, y_{n}\right\}$ dois conjuntos de indeterminadas sobre o anel $B$. Na Seçào 3.4 deste capítulo, provaremos que a funçĩo de Hilbert, $I(B, s, I)$, de um ideal bihomogèneo $I$ do anel $B[X ; Y]$ é um polinomio em $r$ es, para valores suficientemente grandes de $r$ e s. Faremos uso deste resultado para mostrar que no anel local $(A, \mathcal{M})$, de dimensão $d>0$, o comprimento do idcal $I^{r} J^{s}$, onde $I$ e $J$ são dois ideais $\mathcal{M}$-primários é, para valores suficientemente grandes de $r$ e $s$, um polinómio em $r$ e $s$ de grau total $d^{t}=d-1$, onde $d$ é a dimensăo do anel local $A$.

P’ara orientar o leitor, este capítulo, com exceção da última seção, constitui basicamente um detalhamento do artigo [3]. A referencia [11], contém os mesmos resultados do primeiro artigo, com a diferença de que estuda a função de flilbert no caso de apenas um ideal $\mathcal{M}$-primário e trata de outros assuntos. 


\subsection{Resultados Preliminares}

Nesta seção, estudaremos alguns resultados que serão usados posteriormente de uma forma direta on indireta.

Definição 3.2.1: Lim anel $A$ é chamado primário se ele contém no máximo um ideal primo.

Alguns autores definem um ancl com apenas um ideal primo pelo nome de anel Artiniano local. Nós continuaremos a chamar tais anéis de primários.

Note que todo corpo é um inel primário e que todo anel primário é um anel local, desde que todo ideal maximal ś um ideal primo.

Observação 3.2.2: Durante esta seção, usaremos a letra $B$ para denotar um anel primário e a letra A para denotar $11 \mathrm{~m}$ anel local.

Teorema 3.2.3: ([33], pag. 204) Scja $A$ um anel o $I$ um ideal primário de $A$. Então o $B=A / I$ é um anel primário

Lema 3.2.4: Sejam $A$ um anel local, $\mathcal{M}$ seu idcal maximal o $I$ um ideal $\mathcal{M}$-primário. Fntão $B=A / I$ ć um ancl primário Artiniano tendo $\tilde{I}=\mathcal{M} / I$ como seu único ideal primo e, portanto $B$ ć um anel local.

Demonstração: O ideal $\tilde{I}=\mathcal{M} / I$ é primo e maximal. Como o ideal $I$ ć $\mathcal{M}$ primário, o Teorema 3.2.3, garante que o anel $A$ é primário. Além disso, como $A$ tem apenas um ideal primo tem-sc cue dim $A=0$ e como $A$ também ó Voetheriano, segue que é Artiniano

Corolário 3.2.5: Seja $\tilde{I}=\mathcal{M} / I$ o único ideal primo de $B=A / I$. Fntão $\tilde{I}$ pertence ano ideal nulo de $B$.

Demonstração: Sejam $0 \tilde{o}$ o ideal nulo de $B$ e

$$
\tilde{0}-I_{i} \cap \ldots \cap I_{n}
$$

a decomposição primária de 0 em $B$. Então polo Lema 3.2 .4 cada $I_{j}$ é $\dot{I}$-primário en 
$B, j=1, \ldots, n$. Como

$$
\sqrt{\tilde{0}}=\sqrt{I_{i} \cap \ldots \cap I_{n}}=\sqrt{I_{i}} \cap \ldots \cap \sqrt{I_{n}}=\tilde{I}
$$

1emos pelo 'Teorema 1.1.14 (iit), que $\tilde{I}$ pertence ao ideal nulo de $B$ como queríarmos demonstrar.

Definição 3.2.6: Sejam $A$ o $C$ dois ancís tais que $A \subset C$. Fintão dizcmos que $C$ é uma extensäo de A.

Se $I$ é um idcal de $A$ então o conjunto

$$
I^{e}=\left\{\sum_{\text {finita }} a_{i} r_{i}, \text { onde } a_{i} \in I e r_{i} \in C\right\}
$$

é chamado a extensão de $I$ em $C$.

Se $J$ é um ideal do anel $C$, entào a contraçâo de $J$ em $A$ é o conjunto $J \cap A$.

Segue da Definição 3.2.6, que os conjuntos $I^{e} e J \cap A$ são ideais de $C$ e $A$, respectivamente.

Exemplo 3.2.7: Sejam $A$ um ancl $0 x_{1}, \ldots, x_{n}$ indeterminadas sobre 0 anel $A$. Então o anel dos polinomios $A\left[x_{1}, \ldots, x_{n}\right]$ é nma extensão do anel $A$.

Seja $A$ um anel Noetheriano e $\left.A_{i} x_{1}, \ldots, x_{n}\right\}$ o anel dos polinomios do exemplo acima. Denotaremos $A\left[x_{1}, \ldots, x_{n}\right]$ simplesmente por $A[X]$ para simplificar a notação. Um elemento $F\left(x_{1}, \ldots, x_{n}\right)$ será escrito como $F(X)$. Como $A$ ć um anel Noctheriano, segue do Teorema da Base de Hilbert que $A[X]$ também é um anel Noetheriano.

Lema 3.2.8: Sejam $A$ um anel e $I$ um ideal de $A$. Um elemento $F(X)$ pertencente a $A[X]$, pertence ao ideal $I^{e}$ se, e somente se, todos os seus coeficientes estão em $I$.

Demonstração: Se todos os coeficientes de $F(X)$ estão em $I$, então segue da Definição 3.2.6, que $F(X)$ pertence a $I^{e}$.

Reciprocamente, se $F(X)$ pertence a $I^{e}$, então pela Definição 3.2 .6

$$
F(X)-a_{1} F_{1}(X)+\ldots+a_{n} F_{n}(X)
$$


onde cadia $a_{i}$ pertence a $I, i=1, \ldots, n$ e $F_{i}(X)$ sào polinómios em $A[X]$, tais que

$$
F_{i}(X)=\sum_{\text {finila }} a_{i_{1}} \ldots a_{i_{m}} x^{z_{1}} \cdots x^{i_{m}}
$$

Agora como todos os coeficientes de $F_{i}(X)$ estão em $A$, para $i=1, \ldots, n$ e $I$ é um idcal de $A$ segue, por definição de ideal, que todos os coeficientes de $F(X)$ estão cm I.

Corolário 3.2.9: Para qualquer ideal $/$ de $A$ tem-se

$$
I^{e} \cap A=I
$$

Em outras palavras, a contração de um ideal extendido é o próprio ideal.

Demonstração: Com cfcito, se $a \in I$ então $a \in A$ e portanto, pela Definição 3.2.6. temos que $I \subset I^{*}$, e por conseguinte $a \in I^{*} \cap A$. ou seja, $I \subset I^{*} \cap A$.

Por outro lado, se $a \in I^{e} \cap A$. em particular a pertence a $A$, isto é, a pode ser considerado como um polinômio constante cm $A[X]$. Como a pertence a $I^{e}$, seguc do Lema 3.2 .8 que $a \in I$. Isto completa a prova.

O próximo resultado estabelece uma relação entre a decomposição primária de um ideal $I$ de um ancl $A$ e a decomposição primária de sua extensão $I^{e}$, num anel $C$ que f́ extensãolo de $A$.

Corolário 3.2.10: Sejam $I$ um ideal do anel $A$ e $I=I_{1} \cap \ldots \cap I_{n}$ a sua decomposição primária em $A$. Então $I^{e}=I_{1}^{e} \cap \ldots \cap I_{n}^{e}$ é a decomposição primária de $I^{e}$ no anel $A[X\rceil$.

Demonstração: Seja $F(X)$ pertencente a $I^{e}$, então pelo Lema 3.2 .8 temos que todos os seus coeficientes cetâno en $I$, on seja, pertencem a $I_{1} \cap \ldots \cap I_{n}$, mas pelo Corolário 3.2.9, segue que eles pertencem a $I_{1}^{e} \cap \ldots \Gamma I_{n}^{e}$

Para provarmos a inclusão contrária, seja $F(X) \in I_{1}^{f} \cap \ldots \cap I_{n}^{e}$. Então $F(X)$ pertence a cada $I_{i}^{e}$ para $i=1, \ldots$ n e novamente pelo Lema 3.2 .8 , todos os coeficientes cle $F(X)$ estão en $I_{i}$, para $i=1, \ldots, n$. Isto mostra que todos os coeficientes de $F(X)$ estão em $I$, e novamente pelo Lema 3.2 .8 eles também cstão $\mathrm{cm} I^{e}$, $\operatorname{logo} F(X)$ pertence a $I^{\prime \prime}$ e isto completa a demonstração. 
Teorema 3.2.11: ([12], pag. 23) Sujonha que $A$ ć um anol Noetheriano e que $I$ ć un ideal próprio de $A$ e que $J$ seja um ideal arbitrário de $A$. Eintào $(I: J)=1$ sc: e somente se, J nào está contido em nenhum idcal primo pertencente a $l$. Em particular, b é um divisor do zero em $A$ se, e somente se, b pertenee a algum ideal primo de: $A$ que pertence ao ideal nulo de $A$.

Proposição 3.2.12: ([12, pag. 82) Seja $A$ um anel e $C=A[X]$. Se $I$ é um ideal $\mathcal{P}$-prinário do anel $A$, centào $I^{r}$ ó um ideal $\mathcal{P}^{r}$-primário do ancel $C$.

Teorema 3.2.13: Seja

$$
I=I_{1} \cap \ldots \cap I_{n}
$$

a decomposiçáo primária do ideal $I$, onde cada $I_{i}$ é $\mathcal{P}_{i}$-primário para $i=1, \ldots, n$. Então

$$
I^{*}-I_{1}^{:} \cap \ldots \cap I_{n,}^{*}
$$

onde cada $I_{i}^{\ell}$ é $\mathcal{P}_{2}^{e}$-primário para $i=1, \ldots, n$. Além disso, se a primeira decomposição é normal, a segunda também é.

Demonstração: Da Proposição 3.2.12, temos que $I_{i}^{e}$ ć $\mathcal{P}_{i}^{e}$-primário para cada $i=1, \ldots, n$. Agora suponha que

$$
I=I_{1} \cap \ldots \cap I_{n}
$$

6 a decomposição nomal de $I$. Desde que pelo Corolário 3.2.9, $I_{i}^{e} \cap A=I_{i}$ e todos os $I_{i}$ 's são distintos, segue que todos os $I_{i}^{e}$ 's também são distintos. Além disso, a decomposição

$$
I^{e}=I_{1}^{e} \cap \ldots \cap I_{A}^{e}
$$

também ó irredutivel, pois se tivéssemos

$$
I^{\epsilon} \supset \cap_{i \neq j} I_{j}^{\epsilon}
$$

então teríamos

$$
I_{i}=I_{i}^{e} \cap A=I_{i} \supseteq \cap I_{j}^{e}=\cap_{i \neq j} I_{j}
$$

o que contradiria o fato da primeira decomposição sor nomal. 
Teorema 3.2.14: Seja $A$ um anel local, $\mathcal{M}$ seu ideal maximal, $I$ um ideal $\mathcal{M}$ primário e $k=A / \mathcal{M}, \tilde{I}=I / \mathcal{M}$ scu único ideal primo e $\tilde{I}^{\circ}$ sua extensão em $A[x]$. Então $\tilde{I}^{e}$ ó o ínico ideal primo pertenente ao ideal nulo de $A[X]$.

Demonstração: Seja

$$
(0)=I_{1} \cap \ldots \cap I_{n}
$$

a decomposição normal do ideal $0 \mathrm{~cm} A$. Pelo Teorema 3.2.13,

$$
(0)=I_{1}^{e} \cap \ldots \cap I_{n}^{e}
$$

ć a decomposição normal do ideal nulo de $A[X]$. Como $\tilde{I}$ é o único ideal primo de $A$. tremos que

$$
\sqrt{I_{i}}=\tilde{I}
$$

para cada $i=1, \ldots n$, ou seja,

$$
\sqrt{I_{i}^{e}}=\tilde{I}^{\epsilon},
$$

para todo $i=1, \ldots, n$. Portanto, $I^{e}$ é o único ideal primo pertencente ao ideal nulo de $A[X]$, como queríamos demonstrar.

Corolário 3.2.15: Seja $A$ um anel e $A[X]$ o anıl dos polinônios nas indeterminadas $x_{1}, \ldots, x_{n}$ sobre $A$. Então:

i) Todo ideal primo de $A[X]$ contém $I^{e}$;

ii) Se $F(X)$ não pertence a $\tilde{I}^{e}$, então $F(X)$ não ć um divisor do zero $\mathrm{cm} A[X]$.

Demonstração: i) Todo ideal primo $\mathcal{P}$ de $A[X]$ contém o ideal nulo. Seja

$$
\left.\overline{0}=I_{1}^{e}\right\urcorner \ldots \cap I_{r}^{e}
$$

a decomposição normal do ideal nulo em $A[X]$. Então

$$
\tilde{I}^{e}=\sqrt{\overline{0}} \subseteq \sqrt{\mathcal{P}}=\mathcal{P} .
$$

ii) Se $F(X)$ fosse um divisor do zero em $A[X]$, cntáo jelo Teorema 3.2.11, ele pertenceria a algum ideal primo $\mathcal{P}$, pertencente a algum ideal primo do ideal nulo de $A[\mathrm{X}]$, ou seja, ele pertenceria a $\tilde{I}^{e}$, o que contradi\% a nossia hipótesese. 


\subsection{Ideais Bihomogêneos}

Sejam $A$ uma anel local, $\mathcal{M}$ seu único ideal maximal e $I$ um ideal $\mathcal{M}$-primário de $A$. Fntão pelo Tema $3.2 .4 B=A / I$ é um anel Artiniano primário tendo $\mathcal{P}=\mathcal{M} / I$ como seu único ideal primo e portanto $B$ ć urr anel local. No que segue, $B$ é um anel Artiniano primário arbitrário.

Seja $X-\left\{x_{0}, x_{1} \ldots, x_{m}\right\}$ o $Y=\left\{y_{0}, y_{1}, \ldots, y_{n}\right\}$ dois conjuntos de indeterminadas solbre o anel $B$. Então o anel de polinômios $B[X ; Y]$ é um anel bigraduado. Seja $R_{r s} O$ $B$-mórlulo gerado pelos monômios da forma $P Q$ onde $P$ é um monômio de grau $r$ em $X$ e $Q$ é um monomio de grau s em $Y$. Nós dizemos que $P Q$ é um monômio de bigrau $(r, s)$. Seja $R=\oplus_{r, s \geq 0} R_{r s}$ e $R_{r s} R_{a b}=R_{r-a, s+b}$ para todo $r, s, a, b \in \mathbb{N}$. Um elemento de $R_{r s}$ é chamado bihomogêneo de bigrau $(r, s)$. Lm ideal $I \subset R$ gerado por elementos bihomogéneos é chamado um ideal bihomogêneo. Portanto o anel $R / I$ é bigraduado $\mathrm{e}$ as componentes bigraduadas de bigrau $(r, s)$ sendo $A_{r s}=R_{r s} / I_{r s}$ Para simplificar a notação, escreveremos, $F(X ; Y)$ para denotar a forma $F\left(x_{0}, x_{1} \ldots, x_{m} ; y_{0}, y_{1}, \ldots, y_{n}\right)$ em $B[X ; Y]$.

Observação 3.3.1: Em artigos mais recentes, alguns autores, referem-se aos ideais bihomogêneos, chamando-os de ideais bigraduados. Nós continuaremos a chamá-los por bihomogêneos.

Observaçāo 3.3.2: Seja $\mathcal{F}(r, s)$ o $A$-módulo de todas as formas de $B[X, Y]$ de bigrau $(r, s)$. Descde que o $B$-módulo $\mathcal{F}(r, s)$ tem base finita, $\operatorname{dim}_{B} \mathcal{F}(r, s)$, o comprimento de uma série de composição de $B$-submódulos de $\mathcal{F}(r, s)$ é definida e finita. Se $\bar{I}$ é um ideal bihomogêneo de $B: X, Y]$, então $\mathcal{F}(r, s ; \bar{I})$ irá denotar o $B$-submódulo de todas as formas de bigrau $(r, s)$ contidas en $\bar{I}$.

Se $\bar{J}$ é outro ideal bihomogêneo de $B[X, Y]$, cntão temos facilmente que:

$$
\mathcal{F}(r, s ; \bar{I}+\bar{J}) \quad-\quad \mathcal{F}(r, s ; \bar{I})+\mathcal{F}(r, s ; \bar{J})
$$

$$
\mathcal{F}(r, s ; \bar{I} \cap \bar{J})=\mathcal{F}(r, s ; \bar{I}) \cap \mathcal{F}(r, s ; \bar{J})
$$


Lema 3.3.3: Sejam $\bar{I}$ e $\bar{J}$ ideais bihomogêneos de $B[X, Y]$. Então $\operatorname{dim}_{B} \mathcal{F}(r, s ; \bar{I}+\bar{J})+\operatorname{dim}_{B} \mathcal{F}(r, s ; \bar{I} \cap J)=\operatorname{dim}_{B} \mathcal{F}(r, s ; \bar{I})-\operatorname{dim}_{B} \mathcal{F}(r ; s ; \bar{J})$.

Demonstração: Considere as seguintes sequèncias exatas de $B$-módulos:

$$
0 \longrightarrow \mathcal{F}(r, s ; \bar{I}) \cdots \cdots \mathcal{F}(r, s ; \bar{l}+\bar{J}) \longrightarrow \mathcal{F}(r, s ; \bar{I}+\bar{J}) / \mathcal{F}(r, s ; \bar{I})
$$

$$
0 \longrightarrow \mathcal{F}(r, s ; \bar{I} \cap \bar{J}) \longrightarrow \mathcal{F}(r, s ; \bar{J}) \longrightarrow \mathcal{F}(r, s ; \bar{J}) / \mathcal{F}(r, s ; \bar{I} \cap \bar{J}) .
$$

Como dimbs ú uma funçĩo adlitiva temos que:

$$
\begin{aligned}
\operatorname{dim}_{B} \mathcal{F}(r, s ; \bar{I}+\bar{J})-\operatorname{dim}_{B} \mathcal{F}(r, s ; \bar{I}) & =\operatorname{dim}_{B} \mathcal{F}(r, s ; \bar{I}+\bar{J}) / \mathcal{F}(r, s ; \bar{I}) \\
& =\operatorname{dim}_{B}(\mathcal{F}(r, s ; \bar{I})+\mathcal{F}(r, s ; \bar{J})) / \mathcal{F}(r, s: \bar{I}) \\
& =\operatorname{dim}_{B} \mathcal{F}(r, s ; J) / \mathcal{F}(r, s ; I) \cap \mathcal{F}(r, s ; \bar{J}) \\
& -\operatorname{dim}_{B} \mathcal{F}(r ; s ; J) / \mathcal{F}(r ; s ; I \cap J)
\end{aligned}
$$

Da mesma forma obtemos

$$
\operatorname{dim}_{B \beta} \mathcal{F}(r, s ; \tilde{J})-\operatorname{dim}_{B \beta} \mathcal{F}(r, s ; \dot{I} \cap \bar{J})-\operatorname{dim}_{B}(\mathcal{F}(r, s ; \bar{J}) / \mathcal{F}(r, s ; \bar{I} \cap \bar{J}))
$$

Portanto combinando (3.1) e (3.2) temos a fórmula desejada.

Definiçāo 3.3.4: Seja $\vec{I}$ um ideal bihomogeneo de $B[X ; Y]$. Definimos a funç̧̃̃o de Hilbert do ideal $\bar{I}$, denotada por $H(r, s ; \bar{I})$, pela seguinte expressão

$$
H(r, s ; I)=\operatorname{dim}_{B} \int(r, s) \cdots \operatorname{dim}_{B} f(r, s ; \bar{I}) .
$$

Observação 3.3.5: A função de Hilbert $H(r, s ; \bar{I})$, nada mais é do que o comprimento da componente $R_{r s}$ do ancl bigraduado $R / \bar{l}$.

Teorema 3.3.6: Scjam $\bar{l}$ o $\bar{J}$ dois ideais bihomogêneos do ancl $B[X, Y]$. Fintão

$$
H(r ; s ; \bar{I}+\bar{J})=H(r, s ; I)+H(r, s ; \bar{J})-H(r, s ; \bar{I} \cap \bar{J}) .
$$


Demonstração: P’ela Definição 3.3.4, temos:

$$
\begin{gathered}
I I(r, s ; \bar{I}+\bar{J})=\operatorname{dim}_{B} \mathcal{F}(r, s)-\operatorname{dim}_{B} \mathcal{F}(r, s ; \bar{I}+\bar{J}) \\
H(r, s: \bar{I})-\operatorname{dim}_{B} \mathcal{F}(r, s)-\operatorname{dim}_{B} \mathcal{F}(r, s ; \bar{I}) \\
H(r, s ; \bar{J})=\operatorname{dim}_{B} \mathcal{F}(r, s)-\operatorname{dim}_{B} \mathcal{F}(r, s ; \bar{J}) \\
H(r, s ; I \cap \bar{J})-\operatorname{dim}_{B} \mathcal{F}(r, s) \cdots \operatorname{dim}_{B} \mathcal{F}(r, s ; \bar{I} \cap, \bar{J}) .
\end{gathered}
$$

$\log O$

$$
H(r, s ; \bar{I}+\bar{J})-H(r, s ; \bar{I})-H(r, s ; J)-H(r, s ; I \cap J)=0,
$$

on cquivalentemente,

$$
H(r, s ; \bar{I}+\bar{J})=H(r, s ; \bar{I})+H(r, s ; \bar{J})+H(r, s ; \bar{I} \cap \bar{J})
$$

como queríanos demonstrar.

Teorema 3.3.7: Seja $I$ um ideal bihomogêneo próprio de $B[X ; Y]$ e seja $F(X ; Y)$ uma forma de bigrau $(a ; b)$ tal que

$$
(\bar{I}: F(X ; Y))=\bar{I}
$$

Então para $r \geq a$ e $s \geq b$ temos

$$
H(r, s ; \bar{I}+(F))=H(r, s ; \bar{I})-H(r-a, s-b ; \bar{I}) .
$$

Demonstração: Vamos provar inicialmente que $F(X ; Y)$ não é un divisor do zero em $B[X ; Y]$. Se $F(X ; Y)$ fosse um divisor do zcro, então cla pertenceria ao único ideal primo $\tilde{P}$ que pertence ao ideal zero de $B[X ; Y]$, mas como todo ideal primo de $B[X ; Y]$ contém $\tilde{P}$, então $H^{\prime}(X ; Y)$ pertenceria a qualquer ideal primo de $\bar{I}$ o que contraria pelo Teorema 3.2 .11 a nossa hipótese de que $(\bar{I}: F(X ; Y))=\bar{I}$. Portanto $F(X, Y)$ não é um divisor de zero en $B[X ; Y]$. As seguintes igualdades são facilmente verificáveis:

$$
\mathcal{F}(r, s ; I \cap F)-F(X, Y) \mathcal{F}(r \cdots a, s-b ; \tilde{I})
$$




$$
\mathcal{F}(r, s ; F)=F(X, Y) \mathcal{F}(r-a, s-b)
$$

Desde que $F(X: Y)$ não é um divisor de zero, temos os seguintes isomorfismos entre B-módulos.

$$
\mathcal{F}(r, s: I \cap(F)) \cong \mathcal{F}(r-a, s-b ; I)
$$

$$
\mathcal{F}(r, s ;(F)) \cong \mathcal{F}(r-a, s-b)
$$

Portanto

$$
\begin{aligned}
H(r, s ; \bar{I}+(F))= & \operatorname{dim}_{B} \mathcal{F}(r, s)-\operatorname{dim}_{B} \mathcal{F}(r, s ; \bar{I} ;(F)) \\
= & \operatorname{dim}_{B} \mathcal{F}(r, s)-\operatorname{dim}_{B} \mathcal{F}(r, s ; \bar{I})- \\
& \operatorname{dim}_{B} \mathcal{F}(r, s ;(F))+\operatorname{dim}_{B} \mathcal{F}(r, s ; \bar{I} \cap(F)) \\
= & H(r, s ; \bar{I})-H(r-a, r-b ; \bar{I}) .
\end{aligned}
$$

Isto completa a prova do teorema.

Lema 3.3.8: Seja $\mathcal{X}=(\mathcal{P}, X ; Y)$ un ideal bihomogêneo de $B[X ; Y]$. Entào se qualequer ideal bihomogèneo $\bar{I}$ de $B_{i}^{r} \mathrm{X} ; Y$; contém uma potencia de $(\mathrm{X} ; Y)$ entäo $\bar{I}$ ć $\mathcal{X}$-primário.

Demonstração: Como $\mathcal{X}=(\mathcal{P}, X ; Y)$ ć o ideal bihomogêneo maximal de $B[\mathcal{X} ; Y]$ ele contém todo ideal bihomogêneo de $B[X ; Y]$, portanto contém $\bar{I}$. Assim temos que $\sqrt{ } I \subset \mathcal{X}$. Desde quo $\mathcal{P}$ ć um ideal nilpotente, segue que para algum inteiro positivo $k$ temos $\mathcal{P}^{k}=0$. Portanto para csse mesmo inteiro $k$ temos

$$
\mathcal{X}^{k} \subset(X ; Y)
$$

Como $I$ contém uma potencia de $\mathcal{X}$, segue que para algum inteiro positivo $s$ tem-se

$$
\mathcal{X}^{k s} \subset(X ; Y)^{s} \subset I
$$

Portanto temos que $\sqrt{\mathcal{X}^{r}} \mathrm{~s}-\sqrt{\bar{I}}$. como queríamos demonstrar. 
Definição 3.3.9: Iim ideal bihomogeneo próprio $\ddot{I}$ que contém o ideal $(X)^{a}(Y)^{b}$ para inteiros năo negativos a e $b$, é chamado de ideal projetivamente irrelevante do anel $B[X ; Y]$.

Segue do lema anterior que se um ideal bihomogêneo I ć $\mathcal{X}$-primário então ele é necessariamente projetivamente irrelevante.

Lema 3.3.10: Seja $\bar{I}$ um ideal bihomogêneo projetivamente irrelevante de $B[X ; Y]$. Então para valores sufficientemente grandes de $r$ e $s, H(r, s ; I)=0$.

Demonstração: Como I ć um ideal projetivamente irrelevante temos que I contém alguma potência de $(X ; Y)$ e portanto é $\mathcal{X}$-primário pelo Lema 3.3.8. Seguo disto que $\mathcal{X}^{r} \subset I$ \& $\mathcal{X}^{n} \subset I, \log \circ \mathcal{F}(r, s) \subset \mathcal{F}(r, s ; \bar{I})$, como a outra inchusão sempre vale, temos (que $\mathcal{F}(r, s)=\mathcal{F}(r ; s ; \bar{I})$. Portanto $I /(r, s ; \bar{l})=0$.

O próxino teorema afirma que, para valores suficientemente grande de $r$ e $s$, a função de Hilbert $H(r, s ; I)$ de um idcal bihomogeneo $I$, ć um polinômio em $r$ e s.

Teorema 3.3.11: Se $\check{I}$ é um ideal bihomogêneo de $B[X ; Y]$, ertão $H(r, s ; \bar{l})$ é representada, para valores suficientemente grande de $r$ e $s$, por uma expressão da forma

$$
H(r, s ; \bar{I})-\sum_{i+j \ll d-2} a_{i j}\left(\begin{array}{l}
r \\
i
\end{array}\right)\left(\begin{array}{l}
s \\
j
\end{array}\right),
$$

onde $a_{i j}$ são int ciros e d denota a dimensão de $\bar{l}$.

Demonstração: Se $d=0$ ou $d=1$, então $I$ é projetivamente irrelevante, então pelo Lema 3.3.10, para valores suficientemente grandes de $r$ es, $H(r, s ; \ddot{I})=0$,ou seja, $H(r, s ; \bar{I})$ é dado pelo polinômio nulo que tem a expressão (3.4). Fntão o teorema vale para $d=00 d-1$.

Suponha que $d>1$, esuponha que o teorema foi provado para ideais bihomogêneos de dimensões menores. Suponha, além disso, que $\bar{I}$ seja $\mathcal{X}$-primário. Se $I$ ó projetivamente irrelevante, crutão nós já sabemos que $H(r, s ; I)=0$ para valores suficientemente grandes de $r$ e $s$ e, portanto o teorema vale neste caso.

Se $\vec{I}$ não é projetivamente irrelevante, contão existem $x_{i}$ \& $y_{j}$ tais que $x_{i}$ não pertence 
a $\mathcal{I}^{e}$ e $y_{j}$ näo pertenco a $\mathcal{I}^{e}$. Fntăo os irleais $I+\left(x_{i}\right), I+\left(y_{j}\right)$ tem dimensão no máximo $d-1$. P'ortanto polo Teorema 3.3.7 temos que:

$$
H\left(r, s ; \bar{I}+\left(x_{i}\right)\right)=H(r, s ; I)-H(r \cdots 1, s ; I)
$$

$$
H\left(r, s ; I+\left(y_{i}\right)\right)=H(r, s ; I)-H(r, s-1 ; \bar{I}) .
$$

Por hipótese, existem $r_{0}$ o so, tais cues, para $r \geq r_{0}, s \geq s_{0}$,

$$
H\left(r, s ; \bar{I}+\left(x_{2}\right)\right)=\sum_{i+j \leq d-3} b_{i, 3}\left(\begin{array}{l}
r \\
i
\end{array}\right)\left(\begin{array}{l}
s \\
j
\end{array}\right)
$$

$$
I I\left(r, s ; I+\left(y_{i}\right)\right)=\sum_{i, j<d} c_{i j}\left(\begin{array}{l}
r \\
i
\end{array}\right)\left(\begin{array}{l}
s \\
j
\end{array}\right) .
$$

Das equaçöes (3.5) e (3.7) obtemos por somatório

$$
I(r, s ; \bar{I})-H\left(r_{0}, s ; \bar{I}\right)=\sum_{i \rightarrow j<d} b_{i j}\left\{\left(\begin{array}{c}
r+1 \\
i+1
\end{array}\right)-\left(\begin{array}{c}
r_{0}+1 \\
i+1
\end{array}\right)\right\}\left(\begin{array}{l}
s \\
j
\end{array}\right)
$$

Analogamente pelas equações (3.6) • (3.8) temos que

$$
H\left(r_{0}, s ; \bar{I}\right)-H\left(r_{0}, s_{0} ; I\right)=\sum_{i+j \leq d-3} c_{i j}\left\{\left(\begin{array}{c}
s+1 \\
j+1
\end{array}\right)-\left(\begin{array}{c}
s_{0}-1 \\
j+1
\end{array}\right)\right\}\left(\begin{array}{c}
r_{0} \\
i
\end{array}\right) .
$$

Adicionarıdo as equaçôes (3.9) e (3.10), obtemos

$$
\begin{aligned}
H(r, s ; \bar{I})= & H\left(r_{0}, s_{0} \bar{I}\right)+\sum_{i+j<d-3} b_{i j}\left(\begin{array}{c}
r+1 \\
i+1
\end{array}\right)\left(\begin{array}{c}
s \\
j
\end{array}\right)- \\
& \sum_{i+j \leq d-3} b_{i j}\left(\begin{array}{c}
r_{0}+1 \\
i+1
\end{array}\right)\left(\begin{array}{c}
s \\
j
\end{array}\right)+\sum_{i 1, j \leq d-3} c_{i j}\left(\begin{array}{c}
r_{0} \\
i
\end{array}\right)\left(\begin{array}{c}
s+1 \\
j-1
\end{array}\right)- \\
& \sum_{i-j \leqslant d-3} c_{i j}\left(\begin{array}{c}
r_{0} \\
i
\end{array}\right)\left(\begin{array}{c}
s_{0}+1 \\
j+1
\end{array}\right)
\end{aligned}
$$

que tem a forma (3.4). 
Vamos romover a restriçào de que $\bar{I}$ seja primário. Scja

$$
\bar{I}=\bar{I}_{1} \cap \bar{I}_{2} \cap \ldots \cap I_{t}
$$

a decomposição normal de $\bar{I}$ em ideais primários homogêneos. Desde que $\operatorname{dim} \dot{I}=d$ podemos supor que $\operatorname{dim} I_{l}=d$, Seja

$$
\bar{L}=\bar{I}_{1} \cap \bar{I}_{2} \cap \ldots \cap \bar{I}_{l}
$$

Então $\dot{I}=\bar{L} \cap \bar{I}_{l}$, onde $\bar{L}$ tem menos do que $l$ componentes primárias e $\bar{I}_{l}$ é primário de dimensão $d$. Se $l$ - 1 o teorema já foi provado. Suponhamos portanto, que o teorema vale para ideais bihomogêneos com menos do que $l$ componentes primárias. Pcla Equação (3.2) podemos escrever

$$
H(r, s ; \bar{I})=H(r, s ; \bar{L})+H\left(r, s ; \bar{I}_{l}\right)-H\left(r, s ; \bar{I}_{l}+\bar{L}\right) .
$$

Os dois primeiros termos no lado dircito referem-se a ideais com menos do que 1 componentes primárias e o terceiro termo refere-se a um ideal de dimensão menor do que $d$. Portanto todas as funções no lado direito têm a forma (3.4), e portanto, $H(r, s ; I)$ tem a forma (3.4), para valores suficientemente grandes de $r$ e $s$.

Definição 3.3.12: Os coeficientes $a_{i j}$ dos termos da função de Hilbert tais que $i+j=d-2$, onde $d$ é a dimensão do ideal bihomogêneo $\bar{I}$, são chamados os bigruus de $\bar{I}$ : denotados por $e_{i j}(\bar{I})$.

O próximo resultado é uma consequência direta do teorema 3.3 .11

Teorema 3.3.13: Qualquer bigrau $e_{i j}(\bar{I})$ de um ideal bihomogêneo $\bar{I}$ é a soma dos bigraus correspondentes de $\bar{I}$ com a mesma dimensão.

Na Teorema 6 de [3], Bhattacharya afirma que se İ não é projetivamente irrelevante, então cada $\epsilon_{i j}(\bar{I})$ ć maior do que 0 . Na verdade, o que cle prova realmente é que os bigraus são todos não-negativos e que pelo menos um $e_{i j}(\bar{I})$ é de fato maior que zcro. O próximo exemplo esclarece melhor esta questão. 
Exemplo 3.3.14: Se $\bar{I}=0$ então () nào ó um ideal projetivamente irrelevante e temos

$$
H(r, s ; \overline{0})=\left(\begin{array}{c}
r+m \\
m
\end{array}\right)\left(\begin{array}{c}
s+n \\
n
\end{array}\right),
$$

de modo que $e_{m n}(\overline{0}) \neq 0$ e todos os domais $e_{i j}(\overline{0})$ são zero.

\subsection{A Função de Hilbert de dois Ideais $\mathcal{M}$-primários}

Antes de passarmos ao próximo resultado, vamos definir o anel de Rees e fazermos algumas observações que facilit arão a comprensão da demonstração do teorema abaixo.

Scjam $A$ un anel, $I$ um ideal de $A$ e $t$ uma indeterminada sobre $A$. Considere o anel graduado $A: t]$ com a graduação natural ( $\mathbb{N}$-graduado). O conjunto

$$
\left.R .=R_{-}(I, A)-\left\{\sum c_{n} t^{t} \mid c_{n} \in I^{n}\right\}=\oplus_{n} I^{n} t^{n} \subset A^{i} t\right],
$$

ć um subancl de $A[t]$. Se $I=\left(a_{1}, \ldots, a_{r}\right)$, então $R_{+}$pode ser escrito como $R_{1}=$ $A\left[a_{1} l, \ldots, a_{r} l\right]$, de modo que $R_{+}$é Noetheriano se $A$ também for. Agora seja $u=t^{-1}$, c considere $A[t, u]=A\left[t, t^{-1}\right]$ como um $\mathbb{Z}$-anel graduado da maneira usural

$$
R=R(I, A)=R_{+}[u]-\left\{\sum c_{n} l^{n} \mid c_{n} \in I^{n} \text { para } n \geq 0 c c_{n} \in A \text { para } n \leq 0\right\}
$$

$O$ anel $R(I, A) \subset A\left[t, t^{-1}\right]$ é chamado o anel de Rees de $A$ com relação ao ideal I. Mostra-se que $\operatorname{dim} h(I, A)=\operatorname{dim} A+1$. por exemplo, ([9], pag. 122). O anel $R\left(I, t^{-1}\right)=A\left[a_{1} t, \ldots, a_{r}, t, t^{-1}\right]$ é chamado o anel de Rees extendido de $A$ com rolaçâo ao ideal $I$. Mais geralmente, sejam $A$ um anel. $I_{1} \ldots, I_{r}$ ideais de altura positiva de $A$ e $t_{1}, \ldots, t_{r}$ indeterminadas sobre $A$. () anel de Rees $R=A\left[I_{1} t_{1}, \ldots, I_{r} t_{r}\right]$, é o subanel dos polinomios $A\left[t_{1}, \ldots, t_{r}\right]$ consistindo de todas as somas finitas do tipo

$$
\left.\sum a_{\left(\mu_{1}, \ldots, \mu_{r}\right.}\right)_{1}^{h_{1}} \cdots t_{r}^{\mu_{r}}
$$

onde $a_{\left(\mu_{1}, \ldots, \mu_{,}, w_{1}\right.} \subset I_{1}^{\mu_{i}} \ldots I_{r}^{\mu_{r}}$, para todo $\left(\mu_{1}, \ldots, \mu_{r}\right) \in \mathbb{N}^{r}$. 
Teorema 3.4.1: Se J ó um ideal $\mathcal{M}$-primário e I é um ideal próprio qualquer do anel local $(A, \mathcal{M})$. Então para valores suficientemente grande de $r$ es,

$$
\operatorname{dim}_{-4 / J}\left(. J^{r} I^{s} / J^{r} I^{s+1}\right)
$$

é um polinomio em $\rho$ e $\sigma$. Os termos da parte homogênea de grau $d-1$ neste polinômio pode ser escrita como

$$
\left.\frac{1}{(d \cdots 1) !}\left\{\epsilon_{0}(I \mid J) r^{(d}{ }^{1}\right)+\ldots+\left(\begin{array}{c}
d \\
i
\end{array}\right) e_{i}(I \mid J) r^{(d+1} i^{i} s^{i}+\ldots+e_{d}(I \mid J) s^{(d-1)}\right\} .
$$

Demonstração: Sejam $I$ e $J$ dois ideais de $(A, \mathcal{M})$ e $J$ sendo $\mathcal{M}$-primário. Seja $u_{0}, \ldots, u_{m}$ uma base para $I \circ v_{0}, \ldots, v_{n}$ uma base para $J$. Considere o anel de Rees

$$
R=A\left[t_{1} u_{0}, \ldots, t_{1} u_{m} ; t_{2} v_{0}, \ldots, t_{2} v_{n}, t_{2}{ }^{\prime}\right]
$$

onde $l_{1}$ e $l_{2}$ são indeteminadas sobre $A$. Os elementos de $R$ são polinômios da forma

$$
\sum_{i=0}^{s} \sum_{j=-p}^{q} c_{i j} t_{1}^{i} t_{2}^{j}
$$

onde $c_{i j} \in I^{i} J^{j} \mathrm{C} I^{i}=J^{j}=A$ se $i, j$ säo zero ou negativos.

Considere o ideal consistindo de todos os polinomios da forma

$$
\sum_{i=0}^{s} \sum_{j=p}^{q} c_{i j} t_{1}^{i} t_{2}^{j}
$$

onde $c_{i j} \subset I^{i} J^{j+1}$, denotado por $t_{2}{ }^{-1} R$. Portanto $R / t_{2}^{-1} R$ consiste de todos os polinômios da forma

$$
\sum_{i-0}^{s} \sum_{j=-p}^{q} c_{i j} t_{1}^{i} t_{2}^{j}
$$

onde $c_{i j} \in I^{2} J^{j} / I^{i} J^{j+1}$.

Mas, tomando $B=A / J$ temos

$$
R / t_{2}^{-1} R=B\left[\overline{t_{1} u_{0}}, \ldots, \overline{t_{1} u_{m}} ; \overline{t_{2} v_{0}}, \ldots, \overline{t_{2} v_{n}}\right]
$$

descle que $A \cap t_{2}^{-1} R=J$, onde $\overline{T_{1} u_{i}}, \overline{t_{2} v_{j}}$ săo, respectivamente, as imagens de $t_{1} u_{i}$ c $t_{2} v_{j} \mathrm{em} R / t_{2}^{-1} R$ 
Agora considere o homomorfismo

$$
B\left[x_{0}, \ldots, x_{m}: y_{0} \ldots, y_{n}\right] \longrightarrow B\left[\overline{t_{1} u_{0}}, \ldots, \overline{t_{1} u_{m}} ; \overline{t_{2} v_{0}}, \ldots, \overline{t_{2} v_{n}}\right]
$$

onde $x_{0}, \ldots, x_{m} ; y_{0}, \ldots, y_{n}$ são indeterminadas independentes sobre $A / J$.

Se um elemento $F(X ; Y)$ de $B\left[x_{0}, \ldots, x_{m} ; y_{0}, \ldots, y_{n}\right]$ é levado sobre o elemento zero de $B\left[\overline{t_{1} u_{0}}, \ldots, \overline{t_{1} u_{m}}: \overline{t_{2} v_{0}} \ldots, \overline{t_{2} v_{n}}\right]$, então cada forma de $F\left(\mathrm{X}: \mathrm{Y}^{\prime}\right)$ é levado sobre o clemento zero de $B\left[\overline{t_{1} u_{0}}, \ldots, \overline{t_{1} u_{m}}, \overline{t_{2} v_{0}}, \ldots, \overline{t_{2} v_{n}}\right]$. desde que o último é um ancl graduado, e portanto cada forma de $F(X ; Y)$ pertence ao núcleo do homomorfismo. Portanto, o núcleo do homomorfismo acima ć um ideal bihomogêneo $I$, digamos, de $B\left[x_{0}, \ldots, x_{m} ; y_{0}, \ldots, y_{n}\right]$. Portanto

$$
B\left[\overline{t_{1} u_{0}}, \ldots, \overline{t_{1} u_{m}} ; \overline{t_{2} v_{0}}, \ldots, \overline{t_{2} v_{n}}\right] \cong B\left[x_{0}, \ldots, x_{m} ; y_{0}, \ldots, y_{n}\right] / \bar{I}
$$

Então desde que $B$ é um anel Artiniano primário $c \mathcal{I}=\mathcal{M} / J$ é seu único ideal primo, em virtude do isomorfismo acima. temos que

$$
\operatorname{dim}_{B}\left(I^{r} J^{s} / I^{r} J^{s+1}\right)-\operatorname{dim}_{B}[\mathcal{F}(r, s)+\bar{I}] / \bar{I}
$$

onde $\mathcal{F}(r, s)$ é o $B$-módulo de todas as formas de $B[X, Y]$ de bigrau $(r, s)$; assim

$$
\operatorname{dim}_{B}\left(I^{r} J^{s} / I^{r} J^{s+1}\right)=\operatorname{dim}_{B} \mathcal{F}(r, s)-\operatorname{dim}_{B} \mathcal{F}(r, s ; I),
$$

on seja,

$$
\operatorname{dim}_{B}\left(I^{r} J^{s} / I^{r} J^{s \cdot 1}\right)=H(r, s ; \bar{I})
$$

onde $H(r, s ; I)$ é a função de Hilbert do ideal bihomogênco $I$ no ancl de polinômios $B[X, Y]$. Desde que nós já provamos que para valores suficientemente grande do $r, s$, $H(r, s ; \ddot{I})$ ó um polinômio em $r, s$ de grau $d-2$, onde $d$ ć a dimensão de $\bar{I}$ o assim o tcorema está provado.

Observação 3.4.2: Se tivéssemos mostrado que todos os bigraus do polinòmio homogenco de mais alto grau do Teorema 3.3.11, fossem estritamente positivo, cntão todos os coeficientes de

$$
\left.\frac{1}{(d-1) !}\left\{e_{0}(I \mid J) r^{(d} 1\right)+\ldots+\left(\begin{array}{c}
d \\
i
\end{array}\right) c_{i}(I \mid J) r^{(d-1-i)} s^{i}+\ldots+e_{d}(I \mid J) s^{(d-1)}\right\}
$$

também seriam positivos. 
Apresentaremos um exemplo. embora sem detalhamento, para exemplificar uma situação cm que nem todos os coeficientes $e_{i}(I \mid J), i=0, \ldots, d-1$, são maiores que zero.

Exemplo 3.4.3: ([26], pag, 114) Seja $S=k[X, Y, Z, W]]$ ancl das séries das potências formais sobre o corpo $k$ nas indeterminadas $X, Y, Z, W$. Sejam $\mathcal{M}=$ $(X, Y, Z, W), P=(Y, W), Q=(X, Z)$ e $F=(X Y \quad Z W)$ ideais de $S$. Fscreva $R=S /(F), m=\mathcal{M} R \sim q=Q R . \quad$ Neste caso $I=m \cdot J=q \circ c_{0}(m \mid q)=2$, $r_{1}(m \mid q)=1 c_{2}(m \mid q)=0$

Voltamos agora para o principal teorema desta seção.

Teorema 3.4.4: Sejam $I, J$ dois ideais $\mathcal{M}$-primários do anel local $(A, \mathcal{M})$. Entào se $L_{I, J}(\rho, \sigma)$ denota o comprimento do ideal $I^{\rho} J^{\sigma}$. Entrão para valores suficientemente grandes de $\rho$ e $\sigma, L_{I, J}(\rho, \sigma)$ um polinômio em $\rho$ e $\sigma$, cujo grau total ć igual a dimensão do $A$.

Demonstração: Se $A$ ć um ancl primário Artiniano o teorema é trivialmente verdadeiro. Suponhamos entäo que $A$ não é um anel primário. Ponha $B_{1}=A / J, B_{2}=$ $A / I$. Fintão $B_{1}$ o $B_{2}$ são ambos anćis primários e pelo Teorema 3.4.1 temos que

$$
\operatorname{dim}_{B_{1}}\left(I^{r} J^{s} / I^{r} J^{s+1}\right)=H(r, s ; I),
$$

onde $\bar{I}$ ć um ideal bihomogêneo de $B_{1}[X, Y]$ que nâo é projetivamente irrelevante. Do mesmo modo podemos mostrar que

$$
\operatorname{dim}_{B_{2}}\left(I^{r} J^{s} / I^{r+1} J^{s+}\right)=H\left(r, s ; \bar{I}^{\prime}\right),
$$

onde $\bar{I}^{\prime}$ é um ideal bihomogênco de $B_{2}[X, Y]$ que não é projetivamente irrelevante. Portanto existem intciros positivos $\rho$ e $\sigma$ tais que para todos os valores de $r \geq \rho_{0}, s \geq$ $\sigma_{0}$

$$
H(r, s ; \bar{l})-\sum_{i \mid j \leq d} a_{i j}\left(\begin{array}{l}
r \\
i
\end{array}\right)\left(\begin{array}{l}
s \\
j
\end{array}\right)
$$

e

$$
H\left(r, s ; \bar{I}^{\prime}\right)=\sum_{i+j \leq d^{\prime}-2} a_{i j}^{\prime}\left(\begin{array}{l}
r \\
i
\end{array}\right)\left(\begin{array}{l}
s \\
j
\end{array}\right),
$$


onde $a_{i j}$ e $a_{i j}^{\prime}$ são inteiros positivos e $d$ e $d$ são, respectivamente, as dimensões de $\bar{I}$ $\bar{I}^{\prime}$.

$$
\begin{aligned}
& \text { Agora para } \rho>\rho_{0}, \sigma>\sigma_{0} \\
& H_{I, J}(\rho, \sigma)-H_{l, J}\left(\rho_{0}, \sigma_{0}\right)=\sum_{r-\rho_{0}}^{\rho-1} \operatorname{dim}_{B_{2}}\left(I^{r} J^{\sigma_{0}} / I^{r+1} \cdot J^{\sigma_{0}}\right)+\sum_{s=\sigma_{\|}}^{\sigma-1} \operatorname{dim}_{B_{1}}\left(I^{r} J^{s} / I^{r} J^{s+1}\right) \\
& =\sum_{r-\rho_{0}}^{p-1}\left[\sum_{i, j \leq u^{\prime \prime}-2} a_{i i}^{\prime}\left(\begin{array}{c}
r \\
i
\end{array}\right)\left(\begin{array}{c}
s-\sigma_{0} \\
j
\end{array}\right)\right]+ \\
& \sum_{s=\sigma_{0}}^{\sigma}\left[\sum_{i+j \leq d-2} a_{i i}\left(\begin{array}{l}
\rho \\
i
\end{array}\right)\left(\begin{array}{l}
s \\
j
\end{array}\right)\right] \\
& =\sum_{i+j \leq d^{\prime}-2} a_{i j}^{\prime}\left\{\left(\begin{array}{c}
\rho \\
i+1
\end{array}\right)-\left(\begin{array}{c}
\rho_{0} \\
i+1
\end{array}\right)\right\}\left(\begin{array}{c}
\sigma_{0} \\
j
\end{array}\right)+ \\
& \sum_{i+j \leq d-2} a_{i j}\left\{\left(\begin{array}{c}
\sigma \\
j+1
\end{array}\right)-\left(\begin{array}{c}
\sigma_{0} \\
j+1
\end{array}\right)\right\}\left(\begin{array}{c}
\rho \\
i
\end{array}\right) \text {. }
\end{aligned}
$$

ou de outra forma

$$
\begin{aligned}
H_{l, j}(\rho, \sigma)-H_{l, J}\left(\rho_{0}, \sigma_{0}\right)= & \sum_{i+j \leq d-2} a_{i j}\left(\begin{array}{c}
\rho \\
i
\end{array}\right)\left(\begin{array}{c}
\sigma \\
j+1
\end{array}\right)- \\
& \sum_{i+j \leq d-2} a_{i j}\left(\begin{array}{c}
\rho \\
i
\end{array}\right)\left(\begin{array}{c}
\sigma_{0} \\
j+1
\end{array}\right)+ \\
& \sum_{i+j \leq d^{\prime}-2} a_{i, j}^{\prime}\left(\begin{array}{c}
\rho \\
i+1
\end{array}\right)\left(\begin{array}{c}
\sigma_{0} \\
j+k
\end{array}\right) .
\end{aligned}
$$

onde $k$ é uma constante. 
Por outro lado, temos

$$
\begin{aligned}
& H_{I, J}(\rho, \sigma)-I_{I, J}\left(\rho_{0}, \sigma_{0}\right)=\sum_{s-\sigma_{0}}^{\sigma} \operatorname{dim}_{B_{i}}\left(I^{\rho_{0}} J^{s} / I^{\rho_{0}} J^{s-1}\right)+\sum_{r \cdots \rho_{0}}^{\rho-1} \operatorname{dim}_{H_{2}}\left(I^{r} J^{\sigma} / I^{r+1} J^{\sigma}\right) \\
& =\sum_{i=o_{0}}^{\sigma \cdot 1}\left[\sum_{i+j<d-2} a_{i i}\left(\begin{array}{l}
\rho \\
i
\end{array}\right)\left(\begin{array}{l}
s \\
j
\end{array}\right)\right]+ \\
& \sum_{r=\rho_{01}}^{\rho}\left[\sum_{i+j \leq d^{\prime}-2} a_{i i}^{\prime}\left(\begin{array}{l}
r \\
i
\end{array}\right)\left(\begin{array}{l}
\sigma \\
j
\end{array}\right)\right] \\
& -\sum_{i+j \leq d-2} a_{i, j}\left\{\left(\begin{array}{c}
\sigma \\
j+1
\end{array}\right)-\left(\begin{array}{c}
\sigma_{0} \\
j-1
\end{array}\right)\right\}\left(\begin{array}{c}
\rho_{0} \\
i
\end{array}\right) \div \\
& \sum_{i, j \leq d^{\prime}-2} a_{i j}^{\prime}\left\{\left(\begin{array}{c}
\rho \\
i+1
\end{array}\right)-\left(\begin{array}{c}
\rho_{0} \\
i+1
\end{array}\right)\right\}\left(\begin{array}{c}
\sigma \\
j
\end{array}\right) \text {. }
\end{aligned}
$$

ou ainda

$$
\begin{aligned}
I_{l, J}(\rho, \sigma)-I_{l, J}\left(\rho_{0}, \sigma_{0}\right)= & \sum_{i, j \leq d^{\prime}-2} a_{i j}^{\prime}\left(\begin{array}{c}
\rho \\
i \cdot 1
\end{array}\right)\left(\begin{array}{c}
\sigma \\
j
\end{array}\right)- \\
& \sum_{i+j \leq d^{\prime}-2} a_{i j}^{\prime}\left(\begin{array}{c}
\rho_{0} \\
j
\end{array}\right)\left(\begin{array}{c}
\sigma \\
j
\end{array}\right)- \\
& \sum_{i+j \leq d-2} a_{i j}\left(\begin{array}{c}
\rho_{0} \\
i
\end{array}\right)\left(\begin{array}{c}
\sigma_{0} \\
j+1
\end{array}\right)+k,
\end{aligned}
$$

onde $k$ é um inteiro conslante.

Desde que I o J, não são ideais projetivamente irrelevantes nos anéis

$$
B_{1}\left[x_{0}, \ldots, x_{m} ; y_{0}, \ldots, y_{n}\right] \text { e } B_{2}\left[x_{0}, \ldots, x_{m}: y_{0}, \ldots, y_{n}\right]
$$

respectivamente, temos que os bigraus de $I$ o $J$ são maiores ou iguais a zero. Porlanto comparando as duas últimas expressios obtemos $d-d^{\prime}$, isto ce a dimensão do 1 cm $B_{1}\left[x_{0}, \ldots, x_{m} ; y_{0}, \ldots, y_{n}\right]$ ó igual a dimensão de $J \mathrm{~cm} B_{2}\left[x_{0}, \ldots, x_{m} ; y_{0}, \ldots, y_{n}\right]$. Nós 
podemos rescrever a última equação como segue:

$$
\begin{aligned}
H_{I, J}(\rho, \sigma)= & \sum_{i+j<d<2} a_{i, j}\left(\begin{array}{c}
\rho \\
i
\end{array}\right)\left(\begin{array}{c}
\sigma \\
j+1
\end{array}\right)-\sum_{i+j \leq d-2} a_{i j}\left(\begin{array}{c}
\rho \\
i
\end{array}\right)\left(\begin{array}{c}
\sigma_{0} \\
j+1
\end{array}\right)+ \\
& \sum_{i+j \leq d^{\prime}-2} a_{i, j}^{\prime}\left(\begin{array}{c}
\rho \\
i+1
\end{array}\right)\left(\begin{array}{c}
\sigma_{0} \\
j
\end{array}\right)+k,
\end{aligned}
$$

onde $k$ é $\mathrm{um}$ inteiro constante. Ou ainda,

$$
H_{l, j}(\rho, \sigma)=\sum_{i+j \leq d} c_{1}\left(\begin{array}{c}
\rho \\
i
\end{array}\right)\left(\begin{array}{c}
\sigma_{0} \\
j
\end{array}\right),
$$

onde $c_{i j}$ são inteiros e $c_{i j} \geq 0$ se $i+j=d-1$.

Se $I^{\prime}$ e $J^{\prime \prime}$ são outros dois ideais $\mathcal{M}$-primários de $(A, \mathcal{M})$, então pelo Teorema 1.1.10, segue que existem inteiros positivos $m$ o $n$ tais que

$$
I^{\rho} J^{\sigma} \supset I^{\prime m \rho} J^{\prime \prime m \sigma}
$$

$$
I^{\prime \rho} J^{\prime \sigma} \supset I^{n \rho} J^{n \sigma}
$$

Portanto valem

$$
H_{I, \jmath}(\rho, \sigma) \leq H_{I^{\prime}, J^{\prime \prime}}(m \rho, m \sigma)
$$

$$
I_{I^{\prime}, J^{\prime \prime}}(\rho, \sigma) \leq H_{I, J}(n \rho, n \sigma) .
$$

Estas duas equaçóes mostram que o grau de $H_{l, J}(\rho, \sigma)$ é o mesmo que o grau de $H_{I^{\prime}, I^{\prime \prime}}(\rho, \sigma)$. Em particular, se $I=J$, então $H_{I, J}(\rho+\sigma)=H_{I}(\rho+\sigma)$. Mas o grau de $H_{l}(\rho+\sigma)$, que é um polinômio em $\rho+\sigma$, é igual a dimensão do anel local $A$. I'ortianto provamos que o grau do polinômio $H_{\Gamma}(\rho+\sigma)$ é igual a dimensão de $A$.

Vamos reformular o Teorema 3.4.4. Sejam $(A, \mathcal{M})$ um anel local de dimensão positiva $d, I$ o $J$ dois ideais $\mathcal{M}$-primários do ancl local $(\mathcal{A}, \mathcal{M})$. Sejam $L$ a função comprimento e $\mathbb{N}$ o conjunto dos números naturais. Então a funçào

$$
B(r, s): \mathbb{N}^{2} \longrightarrow \mathbb{N}
$$


definida por $B(r, s)=L\left(A / I^{r} y^{s}\right)$, é um polinòmio de grau total $d$, para valores suficientemente grande de $r$ es. Este polinòmio é chamado o polinômio de Bhattacharya de $I$ e $J$. Os termos de grau total $d$ no polinomio de Bhattacharya de $I$ e J tèm a forma

$$
\frac{1}{d !}\left\{\epsilon_{0}(I \mid J) r^{d}+\ldots+\left(\begin{array}{c}
d \\
i
\end{array}\right) \epsilon_{i}(I \mid J) r^{d-i} s^{i}+\ldots+e_{d}(I \mid J) s^{d}\right\} .
$$

Definição 3.4.5: Os clementos

$$
e_{0}(I \mid J), e_{1}(I \mid J), \ldots, e_{d}(I J)
$$

que aparecem no polinômio Bhattacharya de $I$ e $I$, são todos números inteiros, chamados as multiplicidades mixtas dos idcais $I$ e $J$.

No próximo capítulo, trataremos das multiplicidades mixtas de vários ideais $\mathcal{M}$ primários. Por enquanto, estudaremos as multiplicidades mixtas de dois ideais $\mathcal{M}$. primários $I$ e $J$.

\subsection{Multiplicidade Mixta e Dependência Integral}

Nesta secão, estudaremos algumas das propriedades mais elementares das multiplicidades mixtas. Os resultados abordados aqui podem ser encontrados em [28]. A definição de redução de ideal aparece originalmente em [14].

Assim como a multiplicidade $e(I, A)$ de um ideal $\mathcal{M}$-primário foi cstudada exaustivamente por muitos autores, o mesmo aconteceu com as multiplicidades mixtas, sendo esta última, tema atual de pesquisa de vários matemáticos. O nome multiplicidade mixta é devido a B. Teissicr e a J.J. Risler.

Um dos primeiros resultados obtidos sobre os coeficientes do polinômio de Bhattacharya de dois ideais $\mathcal{M}$-primário $I$ e $J$ foi obtido por Recs em ([16], pag. 14). Neste trabalho, Rees provou que se $I$ e $J$ são dois ideais $\mathcal{M}$-primários de una anel local $(A, \mathcal{M})$, cutáo os termos $c_{0}(I \mid J)$ e $e_{d}(I \mid J)$ do polinômio de Bhattacharya de $I$ e $J$ são, respectivamente, iguais a $e(I, A) \odot e(J, A)$, onde $e(I, A)$, denota a multiplicidade do ideal $\mathcal{M}$-primário $I$. 
Vamos provar alguns resultados sobre as multiplicidades mixtas de dois ideais $\mathcal{M}$-primários $I$ e $J$ do ancl local $(A, \mathcal{M})$.

Teorema 3.5.1: Sejam $I$ e $J$ dois ideais $\mathcal{M}$-primários do anel $(A, \mathcal{M}, d)$. Então para quaisquer $r$ es inteiros positivos vale:

$$
e\left(I^{r} J^{s}\right)=e(I) r^{d}+\ldots+\left(\begin{array}{c}
d \\
i
\end{array}\right) e_{i}(I \mid J) r^{d-i} s^{i}+\ldots+e(J) s^{d} .
$$

Demonstração: Pela Fórmula Limite de Samuel temos

$$
e(I ; A)=\lim _{n \rightarrow \infty} \frac{d !}{n^{d}} L\left(A / I^{n} A\right) .
$$

Desde que $I^{r} J^{s}$ é um ideal $\mathcal{M}$-primário, para quaiscuer $r$ e $s$ inteiros positivos vale

$$
\begin{aligned}
e\left(I^{r} J^{s}\right) & =\lim _{n \rightarrow \infty} \frac{d !}{n^{d}} L\left(A /\left(I^{r} J^{s}\right)^{n} A\right) \\
& =\lim _{n+\infty} \frac{d !}{n^{d}} L\left(A /\left(I^{r n} J^{s n}\right) A\right) \\
& =\lim _{n \rightarrow \infty} \frac{d !}{n^{d}} B(r n, s n) \\
& =\lim _{n \rightarrow \infty} \frac{d !}{n^{d}}\left\{\frac{1}{d !} \sum_{i-0}^{d}\left(\begin{array}{c}
d \\
i
\end{array}\right) e_{i}(I \mid J)(r n)^{d i}{ }^{i}(s n)^{i}\right\} \\
& =\lim _{n \rightarrow \infty}\left\{\sum_{i=0}^{d}\left(\begin{array}{c}
d \\
i
\end{array}\right) e_{i}(I \mid J) r^{d}{ }^{i} s^{i}\right\} \\
& =e(I) r^{d}+\ldots+\left(\begin{array}{c}
d \\
i
\end{array}\right) e_{i}(I \mid J) r^{d-i} s^{i}+\ldots+e(J) s^{d},
\end{aligned}
$$

como queríamos mostrar.

Em particular, tomando $r=s=1$ no T'orema 3.5.1, obtemos a multiplicidade do produto de dois ideais $\mathcal{M}$-primários $I$ o $J$.

$$
c(I J)=c(I)+\ldots+\left(\begin{array}{c}
d \\
i
\end{array}\right) e_{i}(I \mid J)+\ldots+c(J) .
$$

Agora, se $I=J$, então para quaisquer inteiros positivos $r$ e $s$, temos

$$
e\left(I^{r+s}\right)=e(I) r^{d}+\ldots+\left(\begin{array}{c}
d \\
i
\end{array}\right) e_{i}(I \mid I) r^{d-i} s^{i}+\ldots+e(I) s^{d} .
$$


Mas por outro lado

$$
e\left(I^{r \cdot s}\right)=(r+s)^{d} e(I)
$$

Segue destas igualdades que

$$
\Theta_{i}(I \mid I)=e(I)
$$

para todo $i=0,1, \ldots d$.

Definição 3.5.2: Seja $(A, \mathcal{M}, d)$ un ancl local. Dados $K, I$ dois ideais de $(A, \mathcal{M}, d)$, dizemos que $K$ é uma redução de $I$ sc:

i) $K \subseteq I$

ii) $K I^{n}=I^{n-1}$ para algum inteiro positivo $n$.

Observação 3.5.3: Note que todo ideal é uma redução dele mesmo, ou seja, todo ideal admite pelo menos uma redução.

Lema 3.5.4: Sejam $I$ e $J$ dois ideais $\mathcal{M}$-primários do anel $A$ tais que $J$ é una reduçäo de $I$, valem as seguintes propriedades;

Se,$J^{r}=I^{r+1}$, entào $J I^{n}=I^{n+1}$, para todo $n \geq r$ e, além disso, $J^{m} I^{r}=I^{r+m}$, para todo inteiro $r \geq 0$.

Demonstração: Segue diretamente da Definição 3.5 .2 e por induşão cm m.

O próximo resultado estabelece uma relação entre a multiplicidade de um idcal $\mathcal{M}$-primário $I$ e qualquer ideal $J$ que seja reduçào de $I$.

Teorema 3.5.5: $\operatorname{Sejam~}(A, \mathcal{M})$ um anel Noetheriano local, $J$ um ideal $\mathcal{M}$-primário e $I$ uma redução de $J$. Então $I$ é $\mathcal{M}$-primário e: além disso: para qualquer $A$-módulo $M$ tem-se

$$
(J, M)=e(I, M)
$$

Em particular, quaisquer duas reduçồ do ideal $J$ têm à mesma multiplicidade

Demonstração: Desde cue $I$ ó uma redução de $J$, então existe um inteiro positivo 
$r$ tal que $I J^{r}=J^{r+1}$ e disto segue que

$$
J^{r+1} \subseteq I \subseteq . J
$$

c aplicando radicais temos que $\sqrt{I}-\mathcal{M}$, isto é, $I$ é um ideal $\mathcal{M}$-primário.

Agora como $I \subset J$, temos que $\epsilon(J \mid M) \leq e(I \mid M)$. Resta então provar a desigualdade contrária. Mas as seguintes desigualdades são verdadeiras

$$
L_{A}\left(M / I^{n+r} M\right) \geq L_{A}\left(M / J^{n+r}\right)=L_{A}\left(M / I^{n} J^{r}\right) \geq L_{A}\left(M / I^{n}\right)
$$

de modo que e $(J \mid M) \geq e(I \mid M)$.

O Teorema 3.5.5 tem uma recíproca parcial. Antes precisamos de uma definição necessária para a compreensão de seu enunciado.

Definição 3.5.6: Seja $(A, \mathcal{M})$ um anel Noetheriano local. Dizemos que $A$ ć um ancl equidimensional se $\operatorname{dim} A / \mathcal{P}=\operatorname{dim} A<\infty$ para todos os ideais primos minimais $\mathcal{P}$ de $A$.

Teorema 3.5.7: ([16], pag. 16) Sejam $A$ um ancl equidimonsional e $I J$ dois ideais $\mathcal{M}$-primários de $A$ tais que $I \subset J$ e $e(I, A)=e(J, A)$, então $I$ é uma redução de $J$.

Corolário 3.5.8: Seja $(A, \mathcal{M}, d)$ un anel local. Se $K$ é uma redução de $I$ então $e(I)-e(K)$

Demonstração: Pclo Teorema 3.5.1, temos

$$
e\left(K I^{s}\right)=e(K)+\ldots+\left(\begin{array}{c}
d \\
i
\end{array}\right) \epsilon_{i}(I \mid J) s^{i}+\ldots+e(I) s^{d}
$$

$$
c\left(I^{1 ! s}\right)-e(I)+\ldots+\left(\begin{array}{c}
d \\
i
\end{array}\right) c_{i}(I \mid I) s^{i}+\ldots+e(I) s^{d} .
$$

Como esta expressão vale para todo $s$, temos $e(K)=e(I)$.

Lema 3.5.9: Se $K$ é uma redução de $I$ e $L$ é uma redução de $J$, então $K^{r} L^{s}$ é uma reduça de $I^{r} J^{s}$ para quaisquer inteciros positivos $r$ es. 
Demonstração: Como $K$ é uma redução de $I$, existe um inteiro positivo $n$ tal que

$$
K I^{n}=I^{n+1}
$$

Da mesma forma, como $L$ é uma redução de $J$, existe $u$ inteiro positivo $m$ tal que

$$
L J^{m}=J^{m+1} .
$$

Assim pelo Lema 3.5.4, temos

$$
K^{r} I^{s}-I^{r 1511} \odot L^{s} \cdot J^{m}=J^{m 1 s \cdot 1} .
$$

Logo

$$
\begin{aligned}
\left(K^{r} L^{s}\right)\left(I^{r} J^{s}\right)^{n m} & =K^{r} I^{r n m} L^{s} \cdot J^{s n m} \\
& =I^{r m n-r} \cdot J^{s m n+s} \\
& =I^{r(m n \cdot 1)} J^{s(m n-1)} \\
& =\left(I^{r} \cdot J^{s}\right)^{m n+1} .
\end{aligned}
$$

Teorema 3.5.10: Se $K e^{\prime}$ uma redução de $I$ e $L$ é mama reduçào de J, entäo

$$
e_{i}(I \mid J)=e_{i}(K \mid L)
$$

para todo $i=1, \ldots d$.

Demonstração: Pelo Lema $3.5 .9, K^{r} L^{s}$ ́ uma reduçào de $T^{r} J^{s}$, para todos inteiros positivos $r$ es. Entä́ temos

$$
e\left(K^{r} L^{s}\right)-e\left(I^{r} J^{s}\right)
$$

Mas

$$
\begin{gathered}
\epsilon\left(K^{r} L^{s}\right)=\sum_{i=0}^{d} \epsilon_{i}(K ! L) r^{d-i} s^{i} \\
\epsilon\left(I^{r} J^{s}\right)=\sum_{i=0}^{d} \epsilon_{i}(I \mid J) r^{d-i} s^{i} .
\end{gathered}
$$

Logo, concluimos que $e_{i}(I \mid J)=e_{i}(K \mid L)$, para todo $i=1, \ldots d$. 
Corolário 3.5.11: Sejam $m$ e $n$ dois inteiros positivos. Fntão para quaisquer inteiros positivos $r$ e $s$ temos

$$
e_{i}\left(I^{m} \mid J^{n}\right)=m^{d-i} n^{i} e_{i}(K \mid L)
$$

Demonstração: Para quaisquer inteiros positivos $r$ e $s$ temos

$$
e\left(I^{m n} \cdot J^{s n}\right)=\sum_{i=0}^{d} e_{i}(I \mid J)(r m)^{d-i}(s n)^{i} .
$$

Por outro lado,

$$
\begin{aligned}
e\left(I^{r m} \cdot J^{s n}\right) & =e\left(\left(I^{m}\right)^{r}\left(J^{n}\right)^{s}\right) \\
& =\sum_{i=0}^{d} e_{i}\left(I^{m} \mid J^{n}\right) r^{d}{ }^{i} s^{i} .
\end{aligned}
$$

Comparando as duas expressões obtcmos

$$
e_{i}\left(I^{m} \mid J^{n}\right)=m^{d-i} n^{i} e_{i}(K \mid L)
$$

Definição 3.5.12: Sejam $x$ um elemento do anel $A$. So $I$ ć um ideal de $A$, dizcmos que $x$ ć inteiro sobre $I$ se ele satisfaz uma equação da forma

$$
x^{n}+a_{1} x^{n-1}+\ldots+a_{n}=0
$$

onde $a_{i} \in I$, para $i=1, \ldots n$.

Lema 3.5.13: Scjam $A$ um anel e $I$ um ideal de $A$. Lim elenento $x \in A$ cinteiro sobre $I$ se, e somente se, para algum $n \geq 0$,

$$
I(I, x)^{n-1}=(I, x)^{n}
$$

Em outras palavras, $I$ é uma redução de $(I, x)$.

Demonstração: Note inicialmente que os ideais $I(I, x)^{n-1}$ o $(I, x)^{n}$, săo realmento distintos, para qualquer valor de $n$, pois $x^{n}$ não pertence a $I(I, x)^{n-1}$, apesar da inclusão $I(I, x)^{n-1} \subset(I, x)^{n}$, sempre valer, independente do valor de $n$. Na verdade, 
$x^{n}$ ó o único elemento de $(I, x)^{n}$ que nào pertence a $I(I, x)^{n}{ }^{1}$. Mas, como por hipótese, $x$ ó inteiro sobra $I$, entäo ele satisfa\% uma equaçào da forma

$$
x^{n}+a_{1} x^{n-1}+\ldots+a_{n}=0
$$

onde $a_{i} \in I$, para $i=1, \ldots n$, ou seja,

$$
x^{n-}-\left(a_{1} \cdot x^{n-1}+\ldots+a_{n}\right) \in(I, x)^{n-1} .
$$

Reciprocamente, seja $x \in A$. Entäo para todo $n \geq 0$, temos $x^{n} \in(I, x)^{n}$. Como, por hipótesce, $I(I, x)^{n}{ }^{1}=(I, x)^{n}$, entào $x^{n} \in I(I, x)^{n}{ }^{1}$, on seja,

$$
x^{n}=\sum_{i-1}^{n} a_{i} b_{i} \text {, onde } a_{i} \in I, b_{i} \in(I, x)^{n-1}, i=1 \ldots, n-1,
$$

e isto mostra que $x$ é integral sobre $I$.

Teorema 3.5.14: Sejam $(A, \mathcal{M})$ um anel Noetheriano local e $I \subset J$ dois ideais de $A$. Então $I$ é unia redução de $J$ se, c somente se, todo clemento de $J$ é integral sobre 1.

Demonstração: Suponha que $I$ seja uma redução de $J$ e seja $x \in J$. Como $I$ ć uma redução de $J$, existe um inteiro $n>0$ tal que $I J^{n}=J^{n+1} \subset I$. Então $x^{n+1} \in J^{n+1} \subset I$. Portanto, $x^{n+1}$ é integral sobre $I$, em particular, $x$ é integral sobre 1 .

Reciprocamente, suponha que todo elemento de J é integral sobre I e suponhla que: $J$ seja gerado pelos elementos $y_{1}, \ldots, y_{r}$, isto ó, $J=\left(y_{1}, \ldots, y_{r}\right)$. Pelo Lema 3.5.13, para cualquer índice $i-1, \ldots, r$, existem inteiros positivos $n_{1} \ldots, n_{r}$, tais que

$$
I\left(I, y_{i}\right)^{n_{i} \quad 1}=\left(I, y_{i}\right)^{n_{t}}
$$

Antes de continuarmos a demonstração, obscrvemos que so um elemento de J é integral sobre 1 , também é integral sobre todo subconjunto $K \subseteq J$ que contenha $I$.

Como $y_{1}$ é integral sobre $I$ tem-se $I\left(I, y_{1}\right)^{n_{1}-1}=\left(I, y_{1}\right)^{n_{1}}$. Mas $y_{2}$ é integral sobre $\left(I, y_{1}\right)$, ou seja, existe um inteiro positivo $n_{2}$ tal que pelo Lema 3.5 .13 ,

$$
\left(I, y_{1}\right)\left(I, y_{1}, y_{2}\right)^{n_{2}} 1=\left(I, y_{1}, y_{2}\right)^{n_{2}}
$$


Acontece que $I\left(I, y_{1}\right)^{n_{1}-1}=\left(I, y_{1}\right)^{n_{1}}$, e multiplicando a Equaçăo (3.14) por $n_{1}-1$ n usando as propriedades de redução, obtemos

$$
\left(I, y_{1}\right)^{n_{1}}\left(I, y_{1}, y_{2}\right)^{n_{2}-1}=\left(I, y_{1}, y_{2}\right)^{n_{2}+n_{3}-1}
$$

Mas por outro lado, temos as seguintes igualdades

$$
\begin{aligned}
\left(I, y_{1}\right)^{n_{1}}\left(I, y_{1}, y_{2}\right)^{n_{2}-1} & =I\left(I, y_{1}\right)^{n_{1}-1}\left(I, y_{1}, y_{2}\right)^{n_{2}-1} \\
& =I\left(I, y_{1}, y_{2}\right)^{n_{2} \cdot n_{1}}{ }^{2}
\end{aligned}
$$

ou seja,

$$
I\left(I, y_{1}, y_{2}\right)^{n_{2}+n_{1}-2}=\left(I, y_{1}, y_{2}\right)^{n_{2}+n_{1}-1} .
$$

Prosscguindo desta mancira, obteromos o resultado.

Vamos provar agora o principal teorema desta seção.

Teorema 3.5.15: Sejam $I$ e $J$ dois ideais $\mathcal{M}$-primários de um anel equidimensional local $(A, \mathcal{M})$ tais que $I \subset J$. Entào $J$ é integral sobre $I$ sce e somente se, $e(I, A)=$ $\ell(J, A)$.

Demonstração: Suponha que $J$ ó integral sobre $I$. Fintão pelo Tocorema $3.5 .14, I$ é uma redução de $J$ e pelo Tcorema $3.5 .5, e(I \mid A)=e(J \mid A)$.

Reciprocamente, suponha que $e(I \mid A)=e(J \mid A)$, cntão pelo Tcorcma 3.5.7, $I$ ć uma redução de $J$. Mas pelo Teorema 3.5.14, $J$ é integral sobre $I$. 


\section{Capítulo 4}

\section{Multiplicidades Mixtas}

\subsection{Introdução}

No final da Seção 3.5, definimos a multiplicidade mixta de dois ideais $\mathcal{M}$-primários $I$ o $J$ do anel local $(A, \mathcal{M})$, ver I) finiçăo 3.4.5. As multiplicidades mixtas para ideais; foram definidas inicialmente por Teissier e Risler em ([23], pag. 302), onde eles estudaram polinomios de Hilbert e seus coeficientes para un conjunto $\mathcal{U}=\left\{I_{1}, \ldots, I_{s}\right\}$, de ideais $\mathcal{M}$-primários arbitrários, usando o que eles chamaram de elementos "suficiontemente gerais". Teissier, mostrou que as multiplicidades mixtas, eram na verdade, a multiplicidade de Samuel de um ideal $\mathcal{M}$-primário, gerado por um conjunto de elementos suficientemente gerais.

\subsection{Resultados Básicos}

Nesta serano, definiremos alguns conceitos necessários para a compreensiáo da próxima seção e também servirá para fixarmos a notação que usiaremos neste capítulo. O principal conceito desta seção, ó o de elemento superficial. Procuraremos mostrar ao leitor, a importância deste conceito, como um artifício para a passagem do passo indutivo de $n \cdots 1$ para $n$. Uma boa referência para este conceito é ([34], pag. 285-296). Uma pergunta que pode surgir ó a seguinte: não é possível imitar a demonstraçào do Teorema 3.4.4. para o caso de mais de dois ideais $\mathcal{M}$-primários?. A resposta é afirmativa, como se porle conferir em [19], mas neste caso, como no Teorema 3.4.4, năa 
conseguimos obter nenhuma informação sobre os coeficientes do termo homogêneo de maior grau do polinômio de Bhatıacharya.

Teorema 4.2.1: ([34], pag. 284) Sejam I um idcal de um anel Noctheriano local $(A, \mathcal{M})$ e $M$ um $A$-módulo Noctheriano. Então a aplicação,

$$
I I(n, I)=L_{A}\left(M / I^{n} M\right)
$$

é um polinômio de grau $d$ em $n$, para $n$ suficientemente grande.

Seja $I$ um ideal $\mathcal{M}$-primário do anel Noetheriano local $(\mathcal{A}, \mathcal{M})$ e seja $x \in I$ Então $I / A x$ também é Noctheriano do anel $A / A x$. O próximo teorema estabeleco uma relação entro os polinômios $H(n, I) \circ H(n, I / A x)$

Teorema 4.2.2: ([34], pag. 285) Seja $I$ um ideal $\mathcal{M}$-primário de um anel local $(A . \mathcal{M})$ c seja $x \in I$. Então

$$
H(n, I / x A)=H(n, I)-L_{A}\left(I^{n}: x A\right) .
$$

Seja $s$ o maior inteiro tal que $x \in I^{s}$, mas $x \notin I^{s+1}$. Note que $I^{n-s} \subseteq\left(I^{n}: A x\right)$. Se pudéssemos provar que $\left(I^{n}: A x\right)$ não é muito diferente de $I^{n-s}$, então teríamos provado que $H(n, I / A x)$ não ć muito diferente de $H(n, I)-H(n-s, I)$, uma situação que é muito útil no caso $s=1$, para provas que usam o processo indutivo.

Definiçāo 4.2.3: Seja $I$ um ideal do anel local $(A, \mathcal{M})$. Dizemos que um elemento $x \in A$ é superficial de ordem $s$ para o ideal $I$. se $x \in I^{s}$ o existe um inteiro positivor tal que

$$
\left(I^{n}: A x\right) \cap I^{c}-I^{n-s},
$$

para todo $n$ suficientemente grande

Observaçāo 4.2.4: A definição de elemento superficial aparecen originalmente em ([20], pag. 182). No caso do corpo ser finito, elementos superficiais de uma detcrminada ordem podem năo existir. Por exemplo, o ideal maximal de

$$
k\{x, y\} /(x y(x+y)),
$$


onde $k$ é um corpo com dois elementos, não possui elemento superficial de ordem 1.

Vejamos como fica o Teorema 4.2.2, com a hipótese de num clemento superficial de orden s para o ideal $I$.

Lema 4.2.5: ([34], pag. 286) Scja I um ideal $\mathcal{M}$-primário do ancl local $(A, \mathcal{M})$ o $x$ um clemento de $A$ superficial de ordem s para $I$. Entio existo 1 m intciro e tal que:

$$
I I(n, I)-H(n, \cdots, l) \leq H(n, I / A x) \leq H(n, I)-H(n-s, I)+H(c, I),
$$

para todo $n$ suficientemente grande.

O Lema 4.2.5 mostra, que se $x$ é um elemento superficial de orden $s$ para um ideal $I$ do ancel $A$, entio para $n$ suficientemente grande, o polinômio $H(n, I / A x)$ difere do polinônio $I I(n, I)-I I(n-s, I)$, apenas por uma constanter.

O segninte lema prova que elementos superficiais realmente existom

Lema 4.2.6: ([34], pag. 286) Scja $I$ um ideal $\mathcal{M}$-primário do anel $(A, \mathcal{M})$. Fntão existe um inteiro s o um elemento $x$ do anel $A$ tal que $x$ é superficial de ordem s para o ideal $I$, isto $e^{\prime}$

$$
\left(I^{n}: A x\right) \cap I^{c}-I^{n-s},
$$

para todo inteiro $n$ suficientemente grande.

Como estamos interessados no passo indutivo, gostaríamos que cxistisse elementos superficiais de ordem 1 para o ideal $I$, e que além disso, esses elementos possuíssem certas propricedades que fossem úteis em provas por indução. O próximo lema é uma respostia para esta pergunta.

Teorema 4.2.7: ([34], pag. 287) Seja $I$ um ideal $\mathcal{M}$-primário do anel local $(A, \mathcal{M})$ c suponha além disso que o corpo residnal $A / \mathcal{M}$ seja infinito. Entäo para cada conjunto finito de ideais $I_{1}, \ldots, I_{r}$, tais que $\sqrt{ } I_{i}^{-} \neq \mathcal{M}$ e cualcuer inteiro positivo $s>0$, existe um elemento $x \in A$ que ś superficial de orden $s$ e que năo pertence a nenhum $I_{i}, i=1, \ldots, r$. 
Em ([34], pag. 291), mostra-se que se I é um ideal $\mathcal{M}$-primário e $x$ é superficial para o idçal $I$, cutão

$$
\operatorname{dim}(A / A x)=\operatorname{dim} A \quad 1
$$

Sejam $\mathbf{i}=\left(i_{1}, \ldots, i_{k}\right)$ e $\mathbf{j}=\left(j_{1}, \ldots, j_{k}\right)$ dois elementos de $\mathbb{Z}^{n}$. Dizemos que $\mathbf{i} \leq \mathbf{j}$ se $i_{p} \leq j_{p}$ para todo $p=1, \ldots$, k. Ainda temos

$$
\left(\begin{array}{l}
\mathbf{i} \\
\mathbf{j}
\end{array}\right)=\left(\begin{array}{l}
i_{1} \\
j_{1}
\end{array}\right) \cdots\left(\begin{array}{l}
i_{k} \\
j_{k}
\end{array}\right)
$$

$$
\left(\begin{array}{l}
\mathbf{i}+\mathbf{e}_{p} \\
\mathbf{j}+\mathbf{e}_{p}
\end{array}\right)=\left(\begin{array}{c}
\mathbf{i} \\
\mathbf{j}+\mathbf{e}_{p}
\end{array}\right)+\left(\begin{array}{l}
\mathbf{i} \\
\mathbf{j}
\end{array}\right) .
$$

onde $\mathbf{e}_{p}$ é o elemento de $\mathbb{Z}^{n}$ que tem 1 na p-ésima componerite o 0 nas demais.

Lema 4.2.8: ([19], pag. 24) Scja $I(\mathbf{i})$ uma funçüo definida para todo $\mathbf{i} \in \mathbb{Z}^{n}$ a suponha que

$$
H(\mathbf{i})-H\left(\mathbf{i}-\mathbf{e}_{p}\right)=\sum_{l=0}^{m} c_{p}\left(\begin{array}{c}
\mathbf{i}+\mathbf{p} \\
\mathbf{p}
\end{array}\right) .
$$

para $c_{p} \in \mathbb{Z}$ e para todo $\mathbf{i} \geq \mathbf{e}_{n}$. Fintão

$$
\begin{aligned}
H(\mathbf{i})= & \sum_{p-0}^{m} c_{p}\left(\begin{array}{c}
\mathbf{i}+\mathbf{p}+\mathbf{e}_{n} \\
\mathbf{p}+\mathbf{e}_{n}
\end{array}\right)+H\left(i_{1} \ldots, i_{n-1}, j_{n}\right)- \\
& \sum_{p=0}^{m}\left(\begin{array}{c}
\tilde{\mathbf{i}}+\tilde{\mathbf{p}} \\
\tilde{\mathbf{p}}
\end{array}\right)\left(\begin{array}{c}
j_{n}+p_{n}-1 \\
p_{n}+1
\end{array}\right) .
\end{aligned}
$$

para todo $\mathbf{i} \geq \mathbf{j}$, onde $\tilde{\mathbf{i}}=\left(i_{1}, \ldots, i_{n-1}\right) \in \mathbb{Z}^{n} \quad$ l.

O somatório $\sum_{p=0}^{m}$, significa que estamos somando sobre todos os elementos de $\mathbb{Z}^{n}$ entre 0 e $m$ com a ordem acima definida.

Vamos definir agora o que entenderemos por urm elemento ser suficientemente geral. 
Definição 4.2.9: Seja $I$ um ideal do anel $(A, \mathcal{M})$. Suponha tambóm que $a_{1} \ldots, a_{l}$ seja $u m$ base minimal de $I$. Suponha que exista um aberto de Zariski $\mathcal{U} \subset k^{\prime \prime}$ tal que so $a-\sum_{i-1}^{\checkmark} \alpha_{i} a_{i} \circ\left(\alpha_{1}+\mathcal{M}, \ldots, \alpha_{l}+\mathcal{M}\right) \in \mathcal{U}$, então a satisfaz uma propriedarle dada. Entäo esta propriedade é chamada suficientemente geral e qualquer elemento de I que satisfaça uma propriedade suficientemente geral é chamado demento suficirntemente geral com relação a esta propriedade.

A vantagem de uma propriedade ser suficientemente geral e que se o corpo $k$ : infinito, é possível encontrar pelo menos um elemento satisfazendo finitas propriedades suficientemente gerais, pois a intersecção finita de abertos de Zariski é ainda um aberto de Zariski.

\subsection{A Definição de Multiplicidade Mixta}

Nesta seção, vamos provar o Teorema 3.4.4, para o caso de um número finito de ideais $\mathcal{M}$-primários. Quando provamos o Teorema 3.4.4, definimos os coeficientes do polinomio homogêneo de maior grau, como as multiplicidades mixtas de dois ideais $\mathcal{M}$-primários, mas não demos informações de quem elas eram realmente, exceto sobre algumas de suas propriedades elementares. Provaremos aqui, que elas são na verdade, a multiplicidade de Samuel de um ideal $\mathcal{M}$ primário, gerado por elementos suficientemente gerais, mais precisamente, de elementos superficiais.

O teorema abaixo, é o principal resultado deste trabalho.

Teorema 4.3.1: ([23], pag. 302) Seja $(A, \mathcal{M}, k, d)$ um anel Noetheriano local, onde $k$ é um corpo infinito. Suponha que $I_{1}, \ldots I_{s}$ são ideais $\mathcal{M}$-prinários e que $M$ seja un $A$-módulo finitamente gerado. Entäo existe un inteiro $c \geq 0$ tal que

$$
L\left(M / I_{1}^{n_{1}} \cdots I_{s}^{n_{s}} M\right)
$$

é um polinomio com coeficientes racionais em $n_{1}, \ldots, n_{s}$, de grau $\operatorname{dim}(M)$, se $n_{i} \geq c$, para cada $i, 1 \leq i \leq s$

O polinômio homogêneo de grau $\operatorname{dim}(M)$ em $L\left(M / I_{1}^{n_{1}} \ldots I_{s}^{n_{s}} M\right)$ pode ser escrito 
como

$$
\sum_{d_{1}+\ldots+d_{s}=\operatorname{dim}(M)} \frac{1}{d_{1} ! \ldots d_{s} !}\left[I^{\left[d_{1} !\right.}, \ldots, I^{\left[d_{s}\right]} ; M\right] n_{1}^{d_{1}} \ldots n_{s}^{d_{s}}
$$

onde $\left.\left[I^{d_{1}}\right], \ldots, I^{\left.d_{s}\right]} ; M\right]$ é um inteiro positivo.

Antes de passarmos a prova do teorema, definiremos os símbolos $\left[I^{\left[d_{1}\right]}, \ldots, I^{\left[d_{s}\right]} ; M\right]$, e enunciaremos um lema que garantirá a existência de elementos suficientemente gerais com propriedades necessárias a demonstraçäo do T'corema 4.3.1. 'Também provaromos vários lemas necessários para a sua demonstração.

Definição 4.3.2: O inteiro positivo $\left[I^{\left[d_{1}\right]}, \ldots, I^{\left[d_{s}\right]} ; M\right]$ é chamado a multiplicidade mirta do módulo $M$ de tipo $\left(d_{1}, \ldots, d_{s}\right)$ com relaçäo aos ideais $I_{1}, \ldots, I_{s}$.

Pretendemos no final desta seção, mostrar que o símbolo $\left[I^{\left.d_{1}\right]}, \ldots, I^{\left[d_{s}\right]} ; M\right]$ é na verdade, a multiplicidade $e\left(I_{1}^{\left[d_{1}, \lambda_{1}\right]}+\ldots+I_{s}^{d d_{s} \cdot \lambda_{s_{1}}}\right)$, de um ideal gerado por $d_{1}$ slementos superficiais de $I_{1}, \ldots, d_{s}$ elementos superficiais de $I_{s}$. Fm resumo, queremos mostrar que

$$
\left[I^{\left[d_{1}\right]}, \ldots, I^{\left[d_{s}\right]} ; M\right]-e\left(I_{1}^{\left[d_{1}, \lambda_{1} \mid\right.}+\ldots+I_{s}^{\left[d_{s}, \lambda_{s}\right]}\right)
$$

Lema 4.3.3: ([23], pag. 303) Sejam $I_{1}, \ldots, I_{s}$ ideais $\mathcal{M}$-primários num ancl Noetheriano local $(A, \mathcal{M}, d, k)$ com corpo residual $k$ infinito e $M$ um $A$-módulo finitamente? gerado. Jefina $I=I_{1}$ e $J=\left(I_{2}, \ldots, I_{s}\right), \mu=\left(n_{2}, \ldots, n_{s}\right)$ e $J^{\mu}=I_{2}^{n \cdot 2} \cdots I_{s}^{n_{s}}$. Então darfo um sistema minimal de geradores $a_{1}, \ldots, a_{l}$ de $I$, existe um aberto não-vazio do Zariski $\mathcal{U} \subset k^{l}$ e inteiros $n_{0}$ e $\mu_{0}=\left(n_{2}^{0}, \ldots, n_{s}^{0}\right)$ tais que para $a=\sum_{i=1}^{l} \alpha_{i} a_{i}$ tem-se para $n \geq n_{0}, \mu \geq \mu_{0} \circ\left(\alpha_{1}+\mathcal{M}, \ldots, \alpha_{l}+\mathcal{M}\right) \subset U$. Além disso, para $a \in I$ nestas condições trmos

$$
\left(I_{1}^{n_{1}} \cdots I_{s}^{n_{s}} M:_{M} a\right) \cap I_{1}^{c} \cdots I_{s}^{n_{s}} M=I_{1}^{\eta_{1}} \cdots I_{s}^{n_{s}} M
$$

Definição 4.3.4: Un elemento $a \in I$ nas condições do Lema 4.3.3 ć chamado um elemento superficial para $I_{1}, \ldots, I_{s}, M$.

Observação 4.3.5: O Lema 4.3.3, mostra que elementos superficiais scmpre exislem, desde que $A$ seja um anel local com corpo residual infinito. O mesmo lema 
também prova que existem $a_{1} \ldots, a_{d}$ elementos do anel $A$ satisfazendo a seguinte condição: os primeiros $d_{1}$ são elementos superficiais pertencentes a $I_{1}, \ldots I_{s}$, e os áltimos $d_{s}$, são elementos superficiais pertencentes a $I_{s}$

() próximo resultado é o Lema de Artin-Rees generalizado.

Lema 4.3.6: (Lema de Artin-Rees Generalizado) ([9], pag. 63) Scjam $I_{1}, \ldots, I_{s}$ ideais $\mathcal{M}$-primários do ancl local $(A, \mathcal{M})$ e $M$ 11m $A$-módulo Noetheriano. Então existem um inteiro $n_{0}$ e $\mu_{0} \in \mathbb{Z}^{n-1}$ tais que

$$
I^{n} . J^{\mu} M \cap a M-I^{n-n_{0}} J^{\mu-\mu_{0}}\left(I^{n_{0}} J^{\mu_{0}} M \cap a M\right)
$$

para todos $n \geq n_{0}$ e $\mu \geq \mu_{0}$

Lema 4.3.7: ([23]. pag. 305) Sejam $I_{1}, \ldots, I_{s}, \mu, \mu_{0}$, como no Lema $4.3 .6 \mathrm{com}$ $\mu \geq \mu_{0}$ e $n$ suficientemente grande. Então temos

$$
\left(I^{n} J^{\mu} M:_{M} a\right)=\left(0:_{M} a\right)+\left(I^{\nu_{1}} J^{\mu} M\right)
$$

e. $v_{1}$ como em (4.1).

Demonstração: Seja $m \in\left(0:_{M} a\right)+I^{n_{1}} . J^{\mu} M$. Funtão $m=m_{1}+m_{2}$, onde $m_{1} \in\left(0:_{M}\right.$ a) e $m_{2} \in I^{n_{1}}, J^{\mu} M$. Segue disto que $a m_{1}=0$. Alérn disto, $I^{v_{1}} J^{\mu} M \subseteq\left(I^{n_{1}} J^{\mu} M:_{M} a\right)$, pela 4.1, o que nos fornece $m_{2}^{\prime} \in\left(I^{n} J^{\mu} M:_{M} a\right)$, ou scja, am $n_{2}^{\prime} \in I^{n} J^{\mu} M$. Portanto,

$$
a m=a m_{1}+a m_{2}^{\prime}-a m_{2}^{\prime} \in I^{n} J^{n} M
$$

ou scja, $m \in\left(I^{n} J^{\mu} M:_{M} a\right)$.

Reciprocamente, seja $m \in\left(I^{n} J^{\mu} M:_{M} a\right)$, isto é, $a m \in I^{n_{1}} J^{\mu} M$. Então pelo Lema 4.3.6, existem $n_{0}$ e $\mu_{0}$ tais que

$$
I^{n} J^{\mu} M \cap a M=I^{n-n_{0}} J^{\mu \mu_{0}}\left(I^{n_{0}} J^{\mu_{0}} M \cap a M\right),
$$

descle que $n \geq n_{0}$ e $\mu \geq \mu_{0}$. Como $I^{n} n_{0}, J^{\mu-\mu_{0}}\left(I^{n_{1}} J^{\mu_{0}} M \cap a M\right) \subseteq I^{n-n_{0}} J^{\mu-\mu_{0}}$, segue que $a m \in I^{n^{n}} n^{n_{1}} J^{\mu-\mu_{0}} a M$. Entäo oscrevemos, $a m=a m^{\prime}$ com $m^{\prime} \in I^{n}{ }^{n_{0}} J^{\mu-\mu_{0}}$. Como 
os ideais $I_{i}$ sào $\mathcal{M}$-primários, se $v$ ó suficientemente grande, tem-se $m^{\prime} \in I^{v-v_{0}} M \subseteq$ $I^{v_{1}} J^{\mu_{0}} \mathrm{M}$. Portanto. para $v$ suficientemente grande, $m^{\prime} \in I^{n_{1}}, J^{\mu} M$. Finalmente

$$
a m=a\left(m-m^{\prime}\right)+a m^{\prime}=a m^{\prime} \in I^{m} J^{\prime \prime} M
$$

desde que $m-m^{\prime} \in\left(0:_{M} a\right)$. Isto prova a igualdade.

Lema 4.3.8: (23], pag. 305) Sejam $I_{1} \ldots, I_{s}$ : como no Lema 4.3 .6 com $\mu$ e $n$ suficientemente grande. Então

$$
\left(I^{n} J_{M}^{\mu} M:_{M} a\right) /\left(I^{n} 1^{1} J^{\mu} M\right) \simeq(0: M a) .
$$

Demonstração: Pclo Lema 4.3.3. $\left(I^{n} J^{\mu} M: M a\right)=\left(0:_{M} a\right)+\left(I^{\nu_{1}} J^{\mu} M\right) \cdot v_{1}$ como em 4.1. Então segue que

$$
\begin{aligned}
\left(I^{n} J^{\mu} M:_{M} a\right) /\left(I^{n-1} J^{\mu} M\right) & \left.\simeq I^{n} J^{\mu} M:_{M} a\right) /\left(\left(I^{n} J^{\mu} M:_{M} a\right) \cap I^{v_{1}} J^{\mu} M\right) \\
& \left.\simeq\left(\left(I^{n} J^{\mu} M:_{M} a\right)+I^{v_{1}} J^{\mu} M\right)\right) / I^{n_{1}} J^{\mu} M \\
& \left.\left.-\left(\left(0:_{M} a\right)+I^{u_{1}} J^{\mu} M\right)+I^{v_{1}} J^{\mu} M\right)\right) / I^{v_{1}} J^{\mu} M \\
& \simeq\left(0:_{M}\right) /\left(I^{v_{1}} J^{\mu} M \cap\left(0:_{M} a\right)\right) .
\end{aligned}
$$

Fixemos $\mu \geq \mu_{0}$. O comprimento do lado dircito da última cquação, depende de $v_{1}$, mas por sua vez, o lado esquerdo não depende. Como $I^{n} J^{\mu} M \cap\left(0:_{M} a\right)$ é decrescente entäo necessariamente devemos ter

$$
I^{i *} J^{n} M \Gamma(0: M a)
$$

como queríamos demonstrar.

Lema 4.3.9: ([23], pag. 301) Sejam $I_{1} \ldots, I_{s}$ ideais $\mathcal{M}$-primários, $n$ e $\mu$ suficientemente grandes. Então a sequêneia

$$
\begin{array}{r}
0 \longrightarrow\left(I^{n} J^{\mu} M:_{M}(t) / I^{n-1} J^{\mu} M \longrightarrow M / I^{n-1} J^{\mu} M \longrightarrow\right. \\
M / I^{n} J^{\mu} M \longrightarrow M / I^{n} J^{\mu} M+a M \longrightarrow 0
\end{array}
$$

c exata, onde a aplicação

$$
M / I^{n}{ }^{1} J^{\mu} M \longrightarrow M / I^{n} J^{\mu} M
$$

é a multiplicaç̃o pelo olemento superficial $a \in I_{1}$. 
Demonstração: Considere a aplicação $\times a: M / I^{n-1} J^{\mu} M \longrightarrow M / I^{n} J^{\mu} M$. Esta aplicação é definida por

$$
m+I^{n-1} J^{\mu} M=a m+I^{n-1} J^{\mu} M .
$$

Ela é bem definida e $\operatorname{Ker}(\times a)=\left(I^{n} J^{\mu} M:_{M} a\right) / I^{n-1} J^{\mu} M$

Por outro lado,

$$
M /\left(I^{n} \cdot J^{\mu} M+a M\right) \simeq\left(M / I^{n} J^{\mu} M\right) / a\left(M / I^{n} J^{\mu} M\right) .
$$

Portanto a aplicaçăo

$$
M / I^{n} J^{\mu} M \longrightarrow\left(M /\left(I^{n} J^{\mu} M\right)\right) / a\left(M / I^{n} J^{\mu} M\right)
$$

definida por

$$
m \longmapsto m+a\left(M /\left(I^{n} J^{\mu} M\right)\right),
$$

ć bem definida e sobrcjetora. Além disso, $\operatorname{Ker}()=\operatorname{Im}(\times a)=a\left(M / I^{n} J^{\mu} M\right)$. Portauto a sequência é de fato exata.

Demonstração do Teorema 4.3.1. A prova ó por indução sobre $d=\operatorname{dim} M$. Se $d=0$, então, por definição, $\operatorname{dim}(A / a m n(M))=0$. Agora $\mathcal{M} / \operatorname{ann}(M)$ é o único idcal primo de $A / \operatorname{ann}(M)$ e pelo fato de $\operatorname{dim}(A / \operatorname{ann}(M))=0$, cle também é seu único idcal primo. Como $\operatorname{dim}(A / \operatorname{ann}(M))=0$, então todo ideal de $A / \operatorname{ann}(M)$ é nilpotente. Segue que existe um inteiro positivo $r$ dependendo de $\mathcal{M}$ tal que $(\mathcal{M} / \text { ann }(M))^{r}=\{0\}$ ( $\{0\}$ ć o submódulo nulo de $A / \operatorname{ann}(M))$.

Se para algumı $n_{1}, \ldots, n_{s}$ tivermos $I_{1}^{n_{1}} \ldots I_{s}^{n_{s}} \subseteq \operatorname{ann}(M)$, então

$$
M /\left(I_{1}^{n_{1}} \cdots I_{s}^{n_{s}} M\right) \simeq M
$$

e portanto, $H_{M}\left(n_{1}, \ldots, n_{s}\right)-L(M)$. Se $I_{1}^{n_{1}} \cdots I_{s}^{n_{s}}$ não está contido em ann $(M)$, então o ideal $\left(I_{1}^{n_{1}} \cdots I_{s}^{n_{s}}+\operatorname{ann}(M)\right) / a n n(M)$ é nilpotente, isto é, cxiste um inteiro positivo, $r^{\prime}$ tal que

$$
\left(\left(I_{1}^{n_{1}} \cdots I_{s}^{n_{s}}+\operatorname{ann}(M)\right) / \operatorname{ann}(M)\right)^{r^{\prime}}=0,
$$

ou seja $\left(I_{1}^{n_{1}} \cdots r I_{s}^{n_{s}}\right)^{r^{\prime}} \subseteq \operatorname{ann}(M)$. Portanto para $n_{1}, \ldots, n_{s}$ suficientemente grande, podemos novamente definir $I_{M}\left(n_{1}, \ldots, n_{s}\right)=L(M)$. 
Suponhamos que o teorema já foi provado para $A$-módulos Noetherianos de dimensão $d-\cdots 1$, isto $́$, se $\operatorname{dim} M=d-1$ e $I_{1}, \ldots, I_{s}$ é um conjunto finito de ideais $\mathcal{M}$-primários do anel local $(A, \mathcal{M})$, então o comprimento $A$-módulo

$$
M / I^{n} J^{\mu} M
$$

ć um polinômio de grau $d-1$. Considere a sequência exata

$$
\begin{array}{r}
0 \longrightarrow\left(I^{n} J^{\mu} M: M a\right) / I^{n-1} J^{\mu} M \longrightarrow M / I^{n}{ }^{1} J^{\mu} M \longrightarrow \\
M / I^{n} J^{\mu} M \longrightarrow M / I^{n} J^{\mu} M+a M \longrightarrow 0
\end{array}
$$

do Lema 1.3.9. Então pela aditividade da função comprimento, temos

$$
\begin{aligned}
L_{A}\left(M / I^{n} J^{\mu} M-a M\right)= & L_{A}\left(M / J^{n} J^{\mu} M\right)- \\
& L_{A}\left(I^{n-1} J^{\mu} M\right) \div L_{A}\left(I^{n} \cdot J^{\mu} M:_{M}(a) / I^{n-1} \cdot J^{\prime \prime} M\right) .
\end{aligned}
$$

Mas, desde que pelo Lema 4.3.8, $\left(I^{n}, J^{\mu} M:_{M} a\right) / J^{n-1} J^{\mu} M \simeq\left(0:_{M} a\right)$, temos da Equação (4.2) que

$$
L_{A}\left(M / I^{n} J^{\mu} M+a M\right)=L_{A}\left(M / I^{n} J^{\mu} M\right)-L_{A}\left(I^{n} 1^{1} J^{\mu} M\right)+L_{A}\left(\left(0:_{M} a\right)\right),
$$

ou seja

$$
H_{M}\left(n_{1}, \ldots, n_{s}\right)-I_{M}\left(n_{1}-1, \ldots, n_{s}\right)=H_{M / a M}\left(n_{1}, \ldots, n_{s}\right)-L_{A}\left(0 i_{M}\right) .
$$

Acontece que $\operatorname{dim}(M / a M)=\operatorname{dim}(M)-1$. Portanto, segue disto e pela hipótese de indução, que $H_{M / a M}\left(n_{1}, \ldots, n_{s}\right)$ é um polinômio de grau $d \cdots 1$, para $n_{1}, \ldots, n_{*}$ suficientemente grande. Como os polinómios $I_{M}\left(n_{1}, \ldots, n_{s}\right) \cdots H_{M}\left(n_{1}-1, \ldots, n_{s}\right)$ diferem do $H_{M / n M}\left(n_{1}, \ldots, n_{s}\right)$ apenas por uma constante, entĩo segue do Lema 4.2 .8 , que $H_{M}\left(n_{1}, \ldots, n_{s}\right)$ é um polinòmio de graı $d$, como queríamos demonstrar.

Varnos agora mostrar que a multiplicidade mixta, é de fato, a multiplicidade do Samuel de um ideal gerado por uma sçuencia de elementos superficiais. Para isto, considere os termos de mais alto gran do polinómio

$$
H_{M}\left(n_{1}, \ldots, n_{s}\right)-I_{M}\left(n_{1}-1, \ldots, n_{s}\right)=H_{M / a M}\left(n_{1}, \ldots, n_{s}\right) .
$$


Então, segue que

$$
\left[I_{1}^{d_{1}}, \ldots, I_{s}^{d_{s}} ; M\right]=\left[I_{1}^{d_{3}} 1^{1}, \ldots, I_{s}^{d_{s}} ; M / a M\right]
$$

Acontece que pelo Lema 4.3.3, á possível encontrar um elemento superficial para $I_{1}, \ldots, I_{s}$ e $\mathrm{M} / \mathrm{a} M$. Fintão prosseguindo teremos encontrado

$$
\left[I_{1}^{d_{1}}, \ldots, I_{s}^{d_{s}} ; M\right]=\left[a_{d} ; M /\left(a_{1}, \ldots, a_{d-1}\right) M\right] .
$$

onde $a_{d}$ pertence a polo menos um dos ideais $I_{1}, \ldots, I_{s}$, o é um elemento superficial para $I_{1}, \ldots, I_{s}$ e $H /\left(a_{1}, \ldots, a_{d-1}\right) M$. Mas, suponhamos que $a_{d} \in I_{1}$, então mostra-se cm ([34], pag. $294 \cdots 295)$ que se dim $A=1$ entâo a multiplicidade do ideal $I_{1}$ e do ideal $a_{d} A$ coincidem. Was $\operatorname{dim}\left(M /\left(a_{1}, \ldots, a_{d-1}\right) M\right)=1$, logo

$$
\begin{aligned}
e\left(a_{d}, M /\left(a_{1}, \ldots, a_{d-1}\right) M\right) & =e\left(. \mid M /\left(a_{1}, \ldots, a_{d-1}\right) M\right)-e\left(0:_{M /\left(a_{1}, \ldots, a_{d-1}\right) M} a_{d}\right) \\
& =L_{4}\left(M /\left(a_{1}, \ldots, a_{d-1}\right) M\right)+L_{A}\left(\left(0:_{M /\left(a_{1}, \ldots, a_{d-1}\right) M} a_{d}\right)\right)
\end{aligned}
$$

que fa a multiplicidade de Samuel de um idcal gerado por uma sequència de elementos superficiais consistindo de $d$ elementos, sendo que os $d_{1}$ são elementos de $T_{1} \ldots$, e os $d_{s}$ são elementos de $I_{s}$

Corolário 4.3.10: ([23], pag. 306) Scjam $(A, \mathcal{M}, k, d), I_{j} 1 \leq j \leq s, M$ como no enunciado do Teorema 4.3.1. Seja $\left.\left(d_{1}, \ldots, d_{s}\right)\right)$ inteiros tais que $d_{1}+\ldots+d_{s}=\operatorname{dim} M$. Então os seguintes símbolos

$$
\begin{aligned}
& e\left(I_{i}: M /\left(I_{1}^{\left[d_{1}, \lambda_{i} ;\right.}+\ldots+\hat{I}_{i}^{\left[d_{i}, \lambda_{i}\right]}+\ldots+I_{s}^{i d_{s}, \lambda_{s}}\right)\right. \\
& e\left(I_{i}^{\left[d_{i}, \lambda_{i}\right]} ; M /\left(I_{i}^{d_{1}, \lambda_{1}}+\ldots i \hat{I}_{i}^{\left.d d_{i}, \lambda_{i}\right]}+\ldots+I_{s}^{\left[d_{s}, \lambda_{s}\right]}\right)\right. \\
& e\left(I_{j} ; M /\left(I_{1}^{\left[d_{1}, \lambda_{i}\right]}+\ldots+\hat{I}_{j}^{\left[d_{j}, \lambda_{j}\right.}+\ldots+I_{s}^{\left[d_{s}, \lambda_{s}\right]}\right),\right.
\end{aligned}
$$

são iguais.

Demonstração: Scgue do comentário acima.

Em particular, se $A$ é um anel Noetheriano local e Cohen- Nacaulay com corpo residual infinito e $I, J$ são dois ideais $\mathcal{M}$-primários, então a aplicação $H: \mathbb{N}^{2}-\rightarrow \mathbb{N}$ definida por

$$
H\left(n_{1}, n_{2}\right)=L_{A}\left(A / I^{n_{1}} J^{n_{2}}\right)
$$


assume os mesmos valores que um polinomio de gran $d=$ dim $A$ e o termo de mais alto grau se escreve como

$$
\vec{H}\left(n_{1}, n_{2}\right)-\frac{1}{d !}\left\{\sum_{i=0}^{d}\left(\begin{array}{c}
d \\
i
\end{array}\right) e_{A}\left(I^{[d-i]}, J^{i}\right) n_{1}^{d-i} n_{2}^{i}\right\}
$$

onde

$$
e_{A}\left(I^{\mid d-i]}, J^{i}\right)=L_{A}\left(A /\left(I^{[d-i}+J^{[i]}\right) A\right)
$$

e $\left.I^{[d-i} \cdot+J^{|i|}\right) A$ é um ideal gerado por uma sequencia de elementos superficiais consistindo de $d-i$ elementos de $I$ e $i$ clementos de $J$ 


\section{Capítulo 5}

\section{Aplicações}

Neste capílulo, daremos uma aplicação geométrica das multiplicidades mixtas. Consideraremos o anel $\mathcal{O}_{n-1}-\mathbb{C}\left\{z_{0}, z_{1}, \ldots, z_{n}\right\}$, das séries convergentes sobre $\mathbb{C}$, localizado no ideal maximal $\mathcal{M}=\left(z_{0}, z_{1}, \ldots, z_{n}\right)$ e continuaremos chamando seu ideal maximal de $\mathcal{M}$.

\subsection{O Número de Milnor}

Para uma melhor compreensão do vocabulário matemático do início desta seção recomentamos [24].

Seja $f:\left(\mathbb{C}^{n+1}, 0\right) \rightarrow \rightarrow \rightarrow(\mathbb{C}, 0)$ um polinômio não constante em $n+1$ variáveis, e $V-f^{1}(0)$ a hipersuperfície complexa definida por $f$, com singularidade isolada no ponto 0 .

Dado $>$ o, consideremos os seguintes subconjuntos de $\mathbb{C}^{n+1}$.

$$
\begin{aligned}
D_{t}= & \left\{z \in \mathbb{C}^{n+1}:|z|<\epsilon\right\} \\
S_{\epsilon}= & \left\{z \in \mathbb{C}^{n+1}:|z|=\epsilon\right\} \\
& K-V \cap S_{\epsilon} .
\end{aligned}
$$

Teorema 5.1.1: (Teorema da Fibração de Milnor) ([24], pag. 5) Seja z um ponto qualquer de $V$ e se $S_{\ell}$ é uma esfera suficientemente pequena centradia em $z$, 


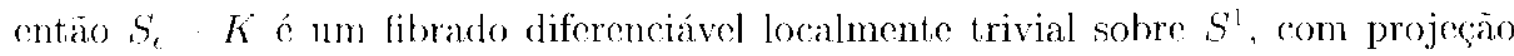
$\phi(z)=f(z) /|f(z)|$ e fibra $F_{\theta}=\phi^{\prime}\left(e^{i \theta}\right)$.

Teorema 5.1.2: ([24], pag. 5) Suponhamos que $z=0$ seja uma singularidade isolada de $f$, então

$$
f: D_{\epsilon} \cap f^{-1}\left(B_{\eta}-\{0\}\right) \rightarrow B_{\eta}-\{0\}
$$

é a projeção de uma fibração localmente trivial, onde $B_{\eta_{l}}=\{w \in \mathbb{C}:|w| \leq \eta\}$.

Observação 5.1.3: ([24], pag. 6) Os resultados do Teorema 5.1.1 o do Teorema 5.1 .2 se correspondem, pois se $w \in \mathbb{C}$ possui módulo suficientemente pequeno, então $f^{\prime}(w) \cap D_{c}$ ć difeomorfo à $F_{y}$.

Teorema 5.1.4: ([24], pag. 7) Cada fibra $F_{0}$ tem o mesmo tipo de homotopia de um bouquet de $S^{n} \mathrm{v} \ldots \mathrm{v} S^{n}$ esforas.

Definição 5.1.5: () número de esferas $S^{\prime \prime}$ do bouquet é chamarlo o número de Milnor de f em o ó denotado por $\mu$.

Definição 5.1.6: Dois germes $(X, 0)$ e $\left(X^{\prime}, 0\right)$ são chamados topologicamente equivalentes se existe um germe de um homeomorfismo

$$
\varphi:\left(\mathbb{C}^{n}, 0\right) \cdots\left(\mathbb{C}^{n}: 0\right)
$$

tal que $\varphi(X, 0)=\left(X^{\prime}, 0\right)$. Também dizemos que os germes $(X, 0)$ e $\left(X^{-1}, 0\right)$ são de: mesmo tipo topolígico.

O préximo teorema mostra que o número de Milnor é $11 m$ invariante topológico.

Teorema 5.1.7: ([23], pag. 295 ou [24], pag. 11) Sejam $(X, 0)$ e $\left(X^{\prime}, 0\right)$ dois germes com singularidade isolada en $\left(\mathbb{C}^{n}, 0\right)$. Se $(X, 0)$ e $\left(X^{\prime}, 0\right)$ sĭo topologicamente cquivalentes então $\mu(X, 0)=\mu\left(X^{\prime}, 0\right)$.

1)ada $f=0$ a equação de um germe $(X, 0)$ com singularidade isolarla: podemos definir o ideal jacobiano de $f$, denotado por $j(f)$, como o ideal de $\mathcal{O}_{n+1}$ gerardo pelas 
derivadas parciais de $f$, isto é,

$$
j(f)=\left(\frac{\partial f}{\partial x_{0}}, \ldots, \frac{\partial f}{\partial x_{n}}\right) .
$$

Temos o seguinte resultado.

Teorema 5.1.8: (24], pag. 23) Scja $j(f)$ o ideal jacobiano de um germe de $\mathcal{O}_{n+1}$. Então dim $\frac{\mathcal{O}_{n}}{j(f)}<\infty$ se, o somente se, 0 ć una singularidade isolada de $f$.

Observaçāo 5.1.9: Vo Capítulo d de [24]. ficou provado que o número de Milnor $\mu(f)$, de um germe com singularidade isolada, e dim $\frac{\mathcal{O}_{n+1}}{j(f)}$ são iguais.

Definição 5.1.10: Definimos o número de Milnor de uma hipersuperfície complexa com singularidade isolada $(X, 0)$ por

$$
\mu(X, 0)=\mu(f)=\operatorname{dim} c \frac{\mathcal{O}_{n}}{j(f)},
$$

ou seja, o núnero de Milnor de um germe com singularidade isolada, nada mais é do que a multiplicinlade do ideal jacobiano de qualquer representante do germe.

Observação 5.1.11: 1) Convém observar que o ideal $j(f)$ é unn idcal $\mathcal{M}$-primário, desde que $f$ tenha singularidade isolada.

2) O número de Mihnor é um objeto geométrico, enquanto dim a $\frac{\mathcal{O}_{n}}{j(f)}$ é um objeto algébrico

\subsection{O Cálculo do Número de Milnor}

Em [24], vários exemplos foram calculados para o número de Milnor. Nesta seção, pretendemos calcular alguns desses exemplos usando as técnicas que apresentamos no Capítulo 2.

Exemplo 5.2.1: Seja $\int:\left(\mathbb{C}^{n+1}, 0\right) \longrightarrow(\mathbb{C}, 0)$, dada por

$$
f\left(z_{0}, z_{1}, \ldots, z_{n}\right)=z_{0}^{2}+z_{1}^{2}+\ldots+z_{n}^{2} .
$$


() ideal $j(f)=\left(2 z_{0}, 2 z_{1}, \ldots, 2 z_{n}\right)$ ć o jacobiano de $f$. Note que $f$ tem uma singularidade isolada em $z=0$. Então

$$
\begin{aligned}
\left(2 z_{0}, 2 z_{1}, \ldots, 2 z_{n,} \mid \mathcal{O}_{n, 11}\right) & \left.-\operatorname{dim} \frac{\mathcal{O}_{n+1}}{\left(2 z_{1}\right)} 2 z_{1}, \ldots, 2 z_{n}\right) \\
& =1 .
\end{aligned}
$$

Exemplo 5.2.2: Seja $f:\left(\mathbb{C}^{2}, 0\right) \longrightarrow(\mathbb{C}, 0)$ dada por

$$
f(x, y)=x^{2} y+y^{4}
$$

O ideal $\left(2 x y, x^{2}+4 y^{3}\right)$ ó o jacobiano de $f$. Note que os conjuntos $\left\{2 x, x^{2}+4 y^{3}\right\}$ e $\left\{y, x^{2}+4 y^{3}\right\}$ são sistemas de multiplicidades em $\mathcal{O}_{2}$. Portanto pelo Teorema 2.4.6, $\left\{2 x y, x^{2}+4 y^{3}\right\}$ também ć um sistema de multiplicidade em $\mathcal{O}_{2}$. Portanto

$$
\begin{aligned}
r\left(2 x y, x^{2}+4 y^{3} \mid \mathcal{O}_{2}\right) & =e\left(2 x, x^{2}+4 y^{3} \mid \mathcal{O}_{2}\right)+c\left(2 y, x^{2}+4 y^{3} \mid \mathcal{O}_{2}\right) \\
& =e\left(y^{3} \mid \mathbb{C}\{y\}\right)+\left(x^{2} \mid \mathbb{C}\{x\}\right) \\
& =5
\end{aligned}
$$

Vamos calcular o número de Milnor de germes determinados por polinòmios quasihomogêneos.

Definição 5.2.3: Sejam $d_{0}, d_{1}, \ldots, d_{n}$ números racionais positivos. Um polinômio $f\left(z_{0}, z_{1}, \ldots, z_{n}\right)$ édito quasi-homogêneo de tipo $\left(d_{0}, d_{1}, \ldots, d_{n}\right)$ se cle pode ser expresso como combinação linear de monômios $z_{0}^{i_{0}} z_{1}^{i_{1}} \cdots z_{n}^{i_{n}}$ tal que

$$
\frac{i_{0}}{d_{0}}+\ldots+\frac{i_{n}}{d_{n}}
$$

O próximo teorema estabelece uma fórmula que permite o cálculo do número de Milnor com relativa facilidade em determinadas situaçós.

Teorema 5.2.4: Scja $f\left(z_{1}, z_{1}, \ldots, z_{n}\right)$ um polinômio quasi-homogeneo de tipo $\left(d_{0}, d_{1}, \ldots, d_{n}\right)$. tendo na origem uma singularidade isolada. Então

$$
\mu=\left(d_{0}-1\right)\left(d_{1}-1\right) \cdots\left(d_{n}-1\right)
$$

Exemplo 5.2.5: Seja $f(x, y)=x^{2}+y^{5}$. Temos que $f$ ó um polinomio quasehomogêneo de tipo $(2,5)$ e entião pelo Tcorema 5.2.4,

$$
\mu-(2-1)(j-1)=4 .
$$




\subsection{Multiplicidades Mixtas e o Número de Milnor}

Scja $f-0, f \in \mathbb{C}\left\{z_{0}, z_{1}, \ldots, z_{n}\right\}=\mathcal{O}_{n+1}$, uma equaçäo para um germe de uma hipersuperfície complexa $\left(\mathrm{X}_{0}, 0\right) \subset(\mathbb{C}, 0)$, isto $e^{\prime}$

$$
\left(X_{0}, 0\right)=\left\{z \in \mathbb{C}^{n+1}: f(z)=0\right\}
$$

ou, de outa forma $\left(X_{i}, 0\right)=f^{-1}(0)$.

Definição 5.3.1: Lim ponto $z \in\left(X_{0}, 0\right)$ é charnado um ponto regular; se existe pelo menos um indice $i, i \in\{0,1, \ldots, n\}$ bal que

$$
\frac{\partial f}{\partial x_{i}}(z) \neq 0
$$

Caso contrário, $z$ é chamado um ponto singular; on unna singularidade do germe $\left(X_{0}, 0\right)$.

Dizcmos que $z \in\left(X_{0}, 0\right)$ é uma singularidade isolada do germe $\left(X_{0}, 0\right)$, se existe uma vizinhanga aberta de $X_{0} \quad\{0\}$ tal cue o germe é regular nesta vizinhança.

Para os nossos objetivos, vamos supor que os germes säo reduzidos.

Seja $C^{(i)}$ o espaço das Grassmanianas do $\mathcal{C}^{n+1}$.

Teorema 5.3.2: ([23], pag. 299) Scja $(X, 0) \subset\left(\mathbb{C}^{n+1}\right)$ um germe de uma hipersuperfícic analítica complexa redurida. Para todo $0 \leq i \leq n+1$ existo uma aberto denso de Zariski $U_{2}^{(i)}$ de $G^{(i)}$ tal que o tipo topológico de $(X \cap H, 0)$ são independentes de $H \subset U_{2}^{(i)}$.

() Teorema 5.3.2, permilo falar do tipo topológico de uma sçăo plana geral $H$ de $(X, 0)$, que chamaremos de subespaço linear geral de dimensäo h. Então pelo 'Teorema 5.1.7. $\mu(\mathrm{X} \cap H, 0)=\mu\left(\mathrm{X} \cap H^{\prime}, 0\right)$ para qualquer subespaço linear genérico $I /$ que passa por 0. Note também que $(X \cap H, 0)$ ć uma hipersuperfície com singularidade isolada c, portanto faz sentido falar do número de Vilnor de um subespaço linear geral de dimensào $h$, para $0 \leq h<n+1$. Segue que o múmero

$$
\mu^{h}(X, 0)=\mu(X \cap I I, 0)
$$

é bem definido. 
Definição 5.3.3: Scjam $(X, 0)$ um germe com singularidade isolada na origem e $H$ um subespaço linear genérico de dimensão $h$, para $0<h<n+1$. Então podemos definir o número de Milnor de $(X\ulcorner H, 0)$ pela igualdade numérica

$$
\mu(X \cap H, 0)=\mu\left(\left.f\right|_{H}\right)=\operatorname{dim} \mathbb{c} \frac{\mathcal{O}_{n+1}}{j\left(\left.f\right|_{I I}\right)} .
$$

A secuência de números

$$
\mu^{*}(X, 0)=\left(\mu^{n+1}(X, 0), \ldots, \mu^{h}(X, 0) \ldots, \mu^{1}(X, 0), \mu^{0}(X, 0)\right),
$$

é chamada a sequência $\mu^{*}$-invariante de $(X, 0)$.

Varmos procurar determinar explicitamente a sequência $\mu^{*}(X, 0)$. Para isto. sejam $I=\mathcal{M}$ e $J=j(f)$, onde $f=0$ e a equação de un germe com singularidade isolada na origem. Considere também a função

$$
K_{X_{0}, 0}: \mathbb{N}^{2} \longrightarrow \mathbb{N}
$$

definida por

$$
K_{\left(X_{0}, 0\right)}(r, s)=\operatorname{dim} c \mathcal{O}_{n+1} / m^{r} j(f)^{s} .
$$

Já sabemos que para valores suficientemente grande de $r$ e s, a funçăo $K_{x_{0}, 0}$ assume os mesmos valores que um polinômio em $r$ e $s$ de grau igual a dimensão de $\mathcal{O}_{n-1}$. Além disso, o polinômio homogêneo de maior grau tem a forma

$$
\bar{K}_{(X, 0)}(r, s)=\frac{1}{(n+1) !} \sum_{i=10}^{n}\left(\begin{array}{c}
n+1 \\
i
\end{array}\right) \tilde{\mu}^{(h), r^{n+1-h} s^{h}}
$$

onde $\tilde{\mu}^{(h)}$ ó a multiplicidade da restrição do ideal jacobiano $j(f)$ a um subespaco linear genérico de $\left(\mathbb{C}^{n+1}, 0\right)$.

Tcissier ([23], pag. 308), mostrou que o ideal jacobiano da restrição da $f$ com um subespaço linear genérico $H$ de dimensão $h$ e a restrição do idcal jacobiano a $H$ têm a mesma multiplicidade, ou seja. $\tilde{\mu}^{(h)}=\mu^{(h)}$. Portanto, podemos rescrever a equaçäo (5.1) da seguinte forma

$$
\bar{K}_{\left(X_{0}, 0\right)}(r, s)=\frac{1}{(n+1) !} \sum_{i=0}^{n}\left(\begin{array}{c}
n+1 \\
i
\end{array}\right) \mu^{(n), r^{n+1-n} s^{h}} .
$$

Portanto temos o seguinte teorema. 
Teorema 5.3.4: ([23], pag. 315) Seja $\left(X_{0}, 0\right) \subset\left(\mathbb{C}^{n+1}, 0\right)$ um germe de uma hipersuperfício analítica complexa com singularidade isolada. Escolhemos as coordenadas $\left(z_{0}, \ldots, z_{n}\right)$ para $\left(\mathbb{C}^{n+1}, 0\right)$ o uma equaçào $f-0$ para $\left(X_{0}, 0\right)$. Consideremos $j(f)=\left(\frac{\partial f}{\partial x_{0}}, \ldots, \frac{\partial f}{\partial x_{x_{n}}}\right), \mathcal{M}=\left(z_{0}, \ldots, z_{n}\right)$ e aplicação $K: \mathbb{N}^{2} \rightarrow \mathbb{N}$ definida por

$$
K\left(n_{1}, n_{2}\right)=L_{A} \mathcal{O}_{n+1} / \mathcal{M}^{n_{i}} j(f)^{n_{2}} .
$$

Então para $n_{1}, n_{2}$ suficientemente grandes, $K$ assume os mesmos valores que um polinômio de grau $n+1$ cujo termo homogêneo de mais alto grau se escreve como

$$
\bar{K}_{\left(X_{0}, 0\right)}(r, s)=\frac{1}{(n+1) !} \sum_{i=0}^{n}\left(\begin{array}{c}
n+1 \\
i
\end{array}\right) \mu^{(h)} r^{n+1-h} s^{h},
$$

onde $\mu^{(h)}=\mu_{x_{11}}^{(h)}\left(X_{0}\right)$. 


\section{Apêndice A}

\section{Localização}

\section{A.1 Localização e Multiplicidades}

Seja $A$ um anel e $S$ um subconjunto multiplicativamente fechado não-vazio tal que $1 \in S$. A relação $\sim \mathrm{em} A \times S$ definida por

$$
(a, s) \sim(b, t) \leftrightarrow(a t-b s) u=0
$$

para algum $u \in S$ é uma relação de equivalência no conjunto $\sim \operatorname{m} A \times S . O$ conjunto de todas as classes de equivalência em $A \times S$ é denotado por $S^{-1} A$. $A$ classe de cquivalência do par $(a, s)$ será denotada por $\frac{a}{s}$. Vamos definir uma estrutura de ancl em $S^{-1} A$. Sejam $\frac{a}{s}$ e $\frac{b}{t}$ duas classes de $S^{-1} A$. Defina a sua soma e seu produto do seguinte modo:

$$
\begin{gathered}
\frac{a}{s}+\frac{b}{t}=\frac{a t+b s}{s t} \\
\frac{a b}{s t}=\frac{a b}{s t} .
\end{gathered}
$$

$O$ anel $S A$ é chamado o anel das fraçóes de $A$ com relação a $S$. A aplicação $f: A \rightarrow S^{-1} A$ definida por $f(a)=\frac{a}{1}$ ce um homomorfismo de anéis, chamado de homomorfismo natural.

Exemplo A.1.1: Scja $\mathcal{P}$ um ideal primo de $A$. Claramente $S-A-\mathcal{P}$ é um subconjunto multiplicativamente fechado de $A: 1 \in S$. Denote $A_{p}$ para o anel $S^{-1} A$. Ap 6 um anel local, chamado a localizaça de $\mathcal{P}$. 
Analogamente, scja $M$ um $A$-módulo. Defina uma relação $\sim \operatorname{em~} M \times S$ como

$$
(m, s) \sim\left(m^{\prime}, s^{\prime}\right) \leftrightarrow \text { existe } t \in S \text { tal que } t\left(s m^{\prime}-s^{\prime} m\right)=0 .
$$

Verifica-se que $\sim$ é uma relação de equivalência no conjunto $M \times S$. O conjunto de todas estas classes é denotado por $S^{-1} M$ e com as operações

$$
\frac{m}{s}+\frac{m^{\prime}}{s^{\prime}}=\frac{s^{\prime} m+s m^{\prime}}{s s^{\prime}}
$$

torna-se um $S^{1}$ A-módulo.

$$
\frac{s m}{s s^{\prime}}=\frac{a m}{s s^{\prime}}
$$

Passaremos de agora em diante a denotar o anel $S^{-1} A$ c o $S^{-1} A$-módulo $S^{-1} \mathrm{M}$ por $A_{S}$ e $M_{S}$, respectivamente.

Scjam $A$ um anel Xoetheriano não necessariamente local, $M$ um $A$-módulo Noctheriano e $\gamma_{1} \ldots, \gamma_{s}$ elementos de $A$ que são um sistema do multiplicidade em $M$. Nós usaremos o símbolo $\mathcal{M}$ para denotar um elemento maximal qualquer de $A$ e $f_{\mathcal{M}}$ : $A \rightarrow A_{\mathcal{M}}$ para denotar o homomorfismo canônico de $A$ na sua localização em $A_{\mathcal{M}}$.

Teorema A.1.2: ([13], pag. 332) Sejam $M$ um $A$-módulo Noetheriano e $\gamma_{1} \ldots, \gamma_{s}$ um sistema de multiplicidade em $M$. Então para todo idcal maximal $\mathcal{M}, M_{\mathcal{M}}$ é um $A_{\mathcal{M}^{-}}$ módulo Noetheriano e $f_{\mathcal{M}}\left(\gamma_{1}\right) \ldots, f_{\mathcal{M}}\left(\gamma_{s}\right)$ é um sistema de multiplicidade em $M_{\mathcal{M}}$. Além disso, existe apenas um númcro finito de ideais maximais $\mathcal{M}$ para os quais $e_{\mathcal{A}_{\mathcal{M}}}\left(f_{\mathcal{M}}\left(\gamma_{1}\right) \ldots, f_{\mathcal{M}}\left(\gamma_{s}\right) \mid M_{\mathcal{M}}\right) \neq 0$ e csse ideais maximais ocorrem entre os ideais maximais que contém o ideal $\left(A n n_{A}(M), \gamma_{1} \ldots, \gamma_{s}\right)$

Teorema A.1.3: (Princípio da Localização) (13]. pag. 333) Sejam A um anel. $M$ um $A$-módulo c $\gamma_{1} \ldots \gamma_{s}$ um sistema de multiplicidade em $M$. Fntăo

$$
e_{M}\left(\gamma_{1} \ldots \gamma_{s} \mid M\right)-\sum_{M} e_{A_{M}}\left(f_{M}\left(\gamma_{1}\right) \ldots f_{M}\left(\gamma_{s}\right) \mid M_{M}\right)
$$

Sejam $A C A^{\prime}$ dois ancicis tais que $A \subset A^{\prime}$. Dizemos que $A^{\prime}$ ć uma extensão de $A$ so a aplicação inclusão $i: A \rightarrow A^{\prime}$ é um homomorfismo de anéis. Neste caso dizemos que $A$ c um subanel de $A^{\prime}$. 
Seja $A^{\prime}$ uma estensão do anel $A$. Dizemos que um elenento $x \in A^{\prime}$ é integral sobre A se ele satisfaz uma relação da forma

$$
x^{n}+a_{1} x^{n-1}+\ldots+a_{n}=0 .
$$

onde $a_{1}, \ldots, a_{n}$ estão $\mathrm{cm} A$ e $n \geq 1$. Note que tedo elemento de $A$ e integral sobre $A$.

Se os únicos elementos de $A^{\prime}$ (que são integrais sobre $A$ são exatamente os elementos de $A$, dizemos entào que $A$ ce integralmente fechlodo sobre $A^{\prime}$.

Suponha que o ancl $A^{\prime}$ seja uma estensão integral do anel $A$. Vamos denotar por $\mathcal{M}_{A^{\prime}}$ um elemento maximal de $A^{\prime}$. $\mathcal{M}_{A^{\prime}} \cap A$ é um ideal maximal de $A$ ([13],pag. 92). Qualquer $A^{\prime}$-módulo é automaticamente um $A$-módulo e, além disso, $A^{\prime} / \mathcal{M}_{A^{\prime}}$ pode ser considerado como nim espaço vetorial sobre o corpo $A / \mathcal{M}_{A^{\prime}} \cap A$ ([13], pag. 333). Denotaremos por $\left[A^{\prime} / \mathcal{M}_{A^{\prime}}: A / \mathcal{M}_{A^{\prime}} \cap A\right]$ a dimentsão deste espaço vetorial.

Teorema A.1.4: (Fórmula da Estensão) ([13], pag. 333) Sejam $A^{\prime}$ uma estensão integral de $A, M$ um $A^{\prime}$-módulo e $\gamma_{1} \ldots, \gamma_{s}$ elementos de $A$. Suponha que

i) $M$ ć Noetheriano tanto como A-módulo quanto $A^{\prime}$-módulo;

ii) $\gamma_{1} \ldots, \gamma$, é um sisterna de multiplicidade quando $M$ for considerado tanto comes A-módulo quanto Á-módulo. Entrăo temos $e_{A}\left(\gamma_{1} \ldots, \gamma_{s} \mid M\right)=\sum_{\mathcal{M}_{A^{\prime}}} e_{{\mathcal{M}^{\prime}}_{A^{\prime}}}\left(f_{\mathcal{M}_{A^{\prime}}}\left(\gamma_{1}\right) \ldots, \int_{\mathcal{M}_{A^{\prime}}}\left(\gamma_{s}\right) \mid M_{\mathcal{M}_{A^{\prime}}}\right)\left[A^{\prime} / \mathcal{M}_{A^{\prime}}: A / \mathcal{M}_{A^{\prime}} \cap A\right]$, onde $f_{\mathcal{M}_{A^{\prime}}}$ denota o homomorfismo canônico de $A^{\prime} \mathrm{em} A_{\mathcal{M}_{A^{\prime}}}^{\prime}$.

Observaçāo A.1.5: 1) Se $\mathcal{M}_{A^{\prime}}$ í tal que $e_{A_{\mathcal{M}_{A^{\prime}}}^{\prime}}\left(f_{\mathcal{M}_{A^{\prime}}}\right)\left(\gamma_{1}\right)=-0$ e arontecer de $\left[A^{\prime} / \mathcal{M}_{A^{\prime}}: A / \mathcal{M}_{A^{\prime}} \cap A\right]=\infty$, então seu produto pode ser considerado como sendo 0 . 2) Se $A=A^{\prime}$, então obtém-se o Tcorema A.1.3 a partir do Teorema A.1.4.

Teorema A.1.6: ([13], pag. 334) Sejam $A$ um ancl local o $\gamma_{1} \ldots \gamma_{s}$ um sistema de multiplicidade $\mathrm{cm} A$. Entrão $s \geq \operatorname{dim} A$. Além disso, $s=\operatorname{dim} A$ se, e somente se, $e_{A}\left(\gamma_{1} \ldots, \gamma_{s} \mid .4\right)>0$

Teorema A.1.7: Sejam A um ancl, $M$ um A-mórlulo Voetheriano e $\gamma_{1} \ldots, \gamma_{s}$ um sistema de multiplicidade em $M$. Suponha que para todo ideal maximal $m$ contendo 
o ideal $\left(\operatorname{Ann}_{A}(M), \gamma_{1} \ldots, \gamma_{s}\right)$ tenhamos $h(M / A n n(M))<s$. Entào

$$
e_{A}\left(\gamma_{1} \ldots, \gamma_{s} \mid M\right)=0
$$

Teorema A.1.8: (Lei Associativa) ([13], pag. 342) Scjam $A$ um ancl e $M$ um $A$ módulo finitamente gerado. Além disso, seja $\gamma_{1} \ldots, \gamma_{s}$ um sistema de multiplicidade no próprio $A$. Se agora $i$ é um int.ciro satisfazendo $0 \leq i \leq s$, então

$$
e_{A}\left(\gamma_{1} \ldots, \gamma_{s} \mid M\right)=\sum_{\mathcal{P}} e_{A_{\mathcal{P}}}\left(f_{\mathcal{P}}\left(\gamma_{1}\right) \ldots, f_{\mathcal{P}}\left(\gamma_{s}\right) \mid M_{\mathcal{P}}\right) e_{A / \mathcal{P}}\left(\pi_{\mathcal{P}}\left(\gamma_{1}\right) \ldots, \pi_{\mathcal{P}}\left(\gamma_{s}\right) \mid R / \mathcal{P}\right)
$$

Aqui $\mathcal{P}$ percorre todos os primos minimais do ideal $\left(\gamma_{1} \ldots, \gamma_{s}\right): f_{\mathcal{P}}: A \rightarrow A_{\mathcal{F}}$ é o homomorfismo canônico de $A$ em $A / \mathcal{p}$ e $\pi_{\mathcal{p}}$ é a projeção canônica de $A \mathrm{~cm} A / \mathcal{P}$. 


\section{Apêndice B}

\section{Teorema de Rees}

\section{B.1 O Complexo de Koszul}

Veste apendice, daremos duas generalizaçóes da noçăo de redução, aplicada a um conjunto de ideais m-primários $\mathcal{U}=\left\{I_{1}, \ldots, I_{s}\right\}$ de um anel Noetheriano local $(A, \mathcal{M})$ de dinensäo d. A primeira é chamada de reduçăo conpleta de $\mathcal{U}$ e a segunda generalização ó chamada de "joint reduction". Nesta última, tomaremos $s=d$. Além disso, definiremos multi-filtração do um conjunto de ideais $m$-primários de um anel Noetheriano local $(A, \mathcal{M})$ e demonstraremos o Teorma 3.4.4 para mais de dois ideais $\mathcal{M}$-primários $I$ e $J$, mostrando (que as multiplicidades mixtas, são números intciros não-negativos. Por todo este apêndice, estaremos supondo que o anel A é Noetheriano de dimensio positiva $d$.

Os resultados deste apêndice, sobre complexo de Koszul, podem ser encontrados em $[13]$

Por um complexo de A-módulos entendemos uma sequência

$$
\cdots \rightarrow M_{n+1} \stackrel{d_{n+1}}{\longrightarrow} M_{n} \stackrel{d_{n}}{\longrightarrow} M_{n-1} \stackrel{d_{n-1}}{\longrightarrow} M_{n-2} \rightarrow \cdots
$$

de A-módulos e homomorfismos, extendidos indefinidamente $\mathrm{em}$ ambas as direcoès, com a propricdade que 


$$
d_{n} \circ d_{n+1}-0
$$

para lodos os valores de $n$. () A-módulo $M_{n}$ será chamado de o módulo componente de grail $n$, ou a n-ésima componente do complexo. Iremos nos referir a $d_{n}$ como o n-ésimo homomorfismo de bordo .

O complexo (B.1), será denotado simplesmente por $(M, d)$, onde $d: M \longrightarrow M 6$ definido por $d\left(x_{n}\right)=d_{n}\left(x_{n}\right)$ para todo $x_{n} \in M$. Note que do (B.2), $d^{2}=0$.

Seja $(M, d)$ un complexo de A-módulos. Defina

$$
Z_{n}(M)-\operatorname{Ker}\left(M_{n} \longrightarrow M_{n-1}\right)=\operatorname{Ker}\left\{d_{n}\right\}
$$

$$
B_{n}(M)=\operatorname{Im}\left(M_{n \mid 1} \longrightarrow M_{n}\right)=\operatorname{Im}\left\{d_{n-1}\right\} .
$$

Os elementos de $Z_{n}(M)$ são chamados os n-ciclos de $M$ e os elementos de $B_{n}(M)$ são chamados os $n$-bordos. Segue de (B.2) que $B_{n}(M) \subseteq Z_{n}(M)$ e, assim podemos definir o módulo quociente

$$
H_{n}(M)=Z_{n}(M) / B_{n}(M)
$$

Chamaremos $H_{n}(M)$ o n-ésimo módulo de homologia do complexo $(M, d)$.

Sejam $\gamma_{1}, \ldots, \gamma_{s}$, onde $s \geq 0$ elementos do anel $A$. Vamos construir o complexo de Koszul do A-módulo $M$ com relaçào aos elementos $\gamma_{1}, \ldots, \gamma_{s}$ do anel $A$, que será denotado por $K\left(\gamma_{1}, \ldots, \gamma_{i} ! M\right)$ ou, às vezes, por $K(\gamma \mid M)$, e tem a forma

$$
0 \rightarrow K_{s}(\gamma \mid M) \stackrel{d_{s}}{\longrightarrow} K_{s} \quad 1(\gamma \mid M) \stackrel{d_{s-1}}{\longrightarrow} \cdots \stackrel{d_{1}}{\longrightarrow} K_{0}(\gamma, M) \rightarrow 0
$$

Para $0 \leq \mu \leq s$, a $\mu$-ésima componente de $K_{\mu}(-\gamma \mid M)$ ć uma soma dircta de $\left(\begin{array}{c}s \\ \mu\end{array}\right)$ cópias de $M$. onde $\left(\begin{array}{c}s \\ \mu\end{array}\right)$ ć o coeficiente binomial usual. Assim quando $s=0$, 
$K_{0}(. \mid M)$ é simplesmente

$$
\cdots \rightarrow 0-\longrightarrow M \longrightarrow 0->\cdots
$$

onde $M$ ocorre como a componente do complexo $K_{0}(. j M)$.

Sejam $T_{i_{1}}, \ldots, T_{i_{\mu}}$ noros símbolos e escreva

$$
K\left(\gamma_{1}, \ldots, \gamma_{s} \mid M\right)=\odot_{i_{1}<\ldots<i_{\mu}} M T_{i_{1}} \cdots T_{i_{\mu}},
$$

onde $i_{1} \ldots, i_{\mu}$ sio inteiros que variann de modo que $0 \leq i_{1}<\cdots<i_{\mu} \leq s$. Assim, quando $0 \leq \mu \leq s, K_{\mu}\left(\gamma_{1}, \ldots, \gamma s, M\right)$ é de fato uma soma direta de $\left(\begin{array}{c}s \\ \mu\end{array}\right)$ cópias de M. Lm elemento arbitrário de $K_{\mu}\left(\gamma_{1}, \ldots \gamma_{s} / M\right)$ tem uma única representaçäo na forma

$$
\sum_{i_{1}<\cdots<i_{\mu}} r_{i_{1} \ldots i_{\mu}} T_{i_{1}} \cdots T_{i_{n}}
$$

onde $r_{i_{1} \ldots i_{k}} \in M$. No caso $=0$, temos $K_{0}\left(\gamma_{1}, \ldots, \gamma_{s} M\right)=M$.

Vamos definir o n-ésimo homonorfismo de bordo

$$
d_{\mu}: K_{\mu}\left(\gamma_{1}, \ldots, \gamma_{s} \mid M\right) \rightarrow K_{\mu}\left(\gamma_{1} \ldots, \gamma_{s} \mid M\right)
$$

para valores de $\mu$ tais que $0<\mu \leq s$.

Suponha que $\mu$ seja como acima e que $i_{1}, \ldots, i_{k}$ sejam inteiros positivos para os quais $1 \leq i_{1}<\cdots<i_{\mu} \leq$ s. Desde cule cada elemento de $k_{\mu}\left(\gamma_{1}, \ldots, \gamma_{s} \mid M\right)$ é expresso de forma única como unta soma de elementos da forma $r T_{i_{1}} \cdots T_{i_{\mu}}$, onde $r \subset M$ podemos definir $d_{\mu}$ en cada parcela desta soma e depois extender por lincaridade. Desta forma defina

$$
d_{\mu}\left(r T_{i_{1}} \cdots T_{i_{\mu}}\right)=\sum_{p=1}^{\mu}(--1)^{p} 1_{\gamma_{i_{p}}} T_{i_{1}} \cdots \hat{T}_{i_{p}} \cdots T_{i_{\mu}}
$$

onde o símbolo $\hat{T}_{i_{p}}$, significa cue $T_{i_{p}}$ foi omitido da parcela $(-1)^{p-1} r T_{i_{1}} \cdots T_{i_{p}} \cdots \Gamma_{i_{p^{\prime}}}$ . Note que $d_{\mu k}$ c um homonorfismo de A-módnlos, $d_{k l} d_{l+1}=0$ c, alón disso,

$$
\cdots \rightarrow K_{\mu ! 1}(\gamma \mid M) \stackrel{d_{\mu+1}}{\longrightarrow} K_{\mu}(\gamma \mid M) \stackrel{d_{\mu}}{\longrightarrow} K_{\mu} \quad 1(\gamma \mid M) \rightarrow \cdots
$$


é realmente um complexo de A-módulos.

Desde que $K\left(\gamma_{1}, \ldots, \gamma_{s}, M\right)$ é um complexo, podemos formar seus módulos de homologia. O $\mu$-ésimo módulo de homologia será denotado por

$$
H_{\mu} K\left(\gamma_{1}, \ldots, \gamma_{s}{ }_{s} M\right)
$$

Caturalmente, $H_{\mu} K\left(\gamma_{1}, \ldots, \gamma_{s} \mathrm{i} M\right)=0$ quando $\mu>0$ e $\mu<0$.

Quando $\mu=1$, temos que $K_{1}\left(\gamma_{1}, \ldots, \gamma_{s} \mid M\right)$ é igual a soma direta de $s$ cópias de M. Então (B.9) asscgura que

$$
d_{1}\left(e T_{i_{1}}\right)=\gamma_{i_{1}} e
$$

Naturalmente um elemento arbitrário de $K_{1}\left(\gamma_{1}, \ldots, \gamma_{s}, M\right)$ tem a forma

$$
\sum_{i=1}^{s} c_{i} T_{i}
$$

onde $\boldsymbol{e}_{i} \in M$ e por (B.11), temos

$$
d_{1}\left(\sum_{i=1}^{s} \epsilon_{i} T_{i}\right)=\gamma_{1} e_{1}+\ldots+\gamma_{s} e_{s} .
$$

Portanto,

$$
\operatorname{Im}\left(d_{1}\right)=\gamma_{1} M+\ldots+\gamma_{s} M
$$

e desde que $K_{1}\left(\gamma_{1}, \ldots, \gamma_{s} \mid M\right)=0$, temos

$$
H_{0} K\left(\gamma_{1}, \ldots, \gamma_{s} \mid M\right)=M /\left(\gamma_{1} M+\ldots+\gamma_{s} M\right) .
$$

Agora, quando $\mu=s$ temos que $K\left(\gamma_{1}, \ldots, \gamma_{s} \mid M\right)$ i a soma direta de $M$ apenas uma vez, isto ć, $K\left(\gamma_{1}, \ldots, \gamma_{s} \mid M\right)=M$. Assim cada elemento de $K\left(\gamma_{1}, \ldots, \gamma_{s} \mid M\right)$ é da forma $e T_{1} \ldots T_{s}$. Então por (B.9),

$$
d_{s}\left(e T_{1} \ldots T_{s}\right)=\sum_{p-1}^{s}(-1)^{p-1} \gamma_{p} e T_{1} \ldots T_{s} .
$$

Desse modo, $d_{s}\left(e T_{1} \ldots T_{s}\right)=0$ se, e somente se, $\gamma_{i} e=0$ (identificamos $e T_{1} \ldots T_{s}$ com c). para $i-1, \ldots, s$. Desta forma, temos

$$
\operatorname{Ker}\left(d_{s}\right)-\left(0:_{M} \gamma_{1} A+\ldots \gamma_{s} A\right)
$$

Portanto desde que $K_{s-1}\left(\gamma_{1}, \ldots, \gamma_{s} \mid M\right)=0$, temos que

$$
H_{s} K\left(\gamma_{1} \ldots \gamma_{s} ; M\right)=\left(0:_{M} \gamma_{1} A+\ldots \gamma_{s} A\right) .
$$


Teorema B.1.1: ([13], pag. 364) Sejam $M$ um $A$-módulo e $\gamma_{1} \ldots, \gamma_{s}$ elementos do anel $A$. Defina $I-\gamma_{1} A+\ldots+\gamma_{s} A$. Entáo cada $\gamma_{i}$ anula os módulos de homologia $H_{\mu} K\left(\gamma_{1}, \ldots, \gamma_{s} \mid M\right)$ do complexo de Kośzul de $M$ com rolação a $\gamma_{1}, \ldots, \gamma_{s}$. Fm particular, I amula todos os módulos de homologia $H_{\mu} K\left(\gamma_{1}, \ldots, \gamma_{s} \mid M\right)$.

Teorema B.1.2: ([13], pag. 369) Scjam $M$ um $A$-módulo Noetheriano e $\gamma_{1}, \ldots, \gamma_{s}$ elementos do anel $A$ tais que

$$
L\left(M /\left(\gamma_{1} M+\ldots+\gamma_{s} M\right)\right.
$$

tem comprimento finito. Fntão, $H_{\mu} K\left(\gamma_{1}, \ldots, \gamma_{s} \mid M\right)$ tem comprimento finito para todo $\mu \in\{0, \ldots, s\}$.

Observação B.1.3: O Teorema (B.1.2), diz que se $\gamma_{1}, \ldots, \gamma_{s}$ é um sistema do multiplicidade $\mathrm{cm} M$, então $I_{\mu} K\left(\gamma_{1}, \ldots, \gamma_{s} \mid M\right)$ tem comprimento finito para todo $\mu \in\{0, \ldots, s\}$.

Seja $M$ um $A$-módulo Noetheriano e $\gamma_{1} \ldots, \gamma_{s}$ un sistema de multiplicidado em M. Pelo Teorema B.1.2, cada módulo de homologia $H_{\mu} K\left(\gamma_{1}, \ldots, \gamma_{s} \mid M\right)$, tem comprimento finito. Portanto podemos definir

$$
\mathcal{X}\left(\gamma_{1}, \ldots, \gamma_{s} ! M\right)=\sum_{\mu}(-1)^{\mu} L_{A}\left(I_{\mu} K\left(\gamma_{1}, \ldots, \gamma_{s} \mid M\right)\right) .
$$

Note que esta soma é finita, desde que o somando é zero se $\mu>s$ ou $\mu<0$. Iremos mostrar fue

$$
\mathcal{X}\left(\gamma_{1}, \ldots, \gamma_{s} \mid M\right)=\epsilon_{A}\left(\gamma_{1}, \ldots, \gamma_{s} \mid M\right)
$$

Observe que se $s=0$, entăo da Equação (B.12), concluímos que

$$
\mathcal{X}(. \mid M)=e_{A}(. \mid M)
$$

Antes de provarmos este resultado, vamos fazer algumas observaçós e enunciaremos alguns teoremals.

Suponha que $(M, d)$ é um complexo de $A$-módulos tal que todos os módulos componentes tenham comprimento finito e no máximo um número finito deles são não-nulos. 
Então

$$
\sum_{n}(-1)^{n} L_{\Lambda}\left(M_{n}\right)
$$

está bem definida. Fsta soma ó chamada a característica de L̇uler-Poincaré do complexo $(M, d)$.

Os próximos teoremas são essenciais na demonstração de (B.13) e na comprecnsão do que faremos na próxima scção.

Teorema B.1.4: ([13], pag. 355) Sejam $M$ um A-módulo e $\gamma_{1}, \ldots, \gamma_{s}$ um sistema de multiplicidade em $M$. Considere o complexo de Ḱoszul $K\left(\gamma_{1}, \ldots, \gamma_{s} \mid M\right)$. Então a sequência

$$
0 \cdots H_{s} K(\gamma \mid M) \cdots H_{s}, K(\gamma \mid M) \quad>\cdots \longrightarrow H_{1} K(\gamma \mid M) \longrightarrow H_{0} K(\gamma \mid M) \longrightarrow 0
$$

é exata.

O complexo do Teorema (B.1.4), é chamado o complexo de homologia do complexo $K\left(\gamma_{1}, \ldots, \gamma_{s} \mid M\right)$.

Sejam $M^{\prime}, M$ e $M^{\prime \prime}$ complexos de A-módulos e seja $\phi: M^{\prime} \longrightarrow M$ e $\psi^{\prime}: M \longrightarrow M^{\prime \prime}$ aplicações de complexos. Ijizcmos que

$$
0 \rightarrow M^{\prime} \stackrel{\phi}{\longrightarrow} M \stackrel{\psi}{\longrightarrow} M^{\prime \prime} \rightarrow 0
$$

é uma sequência exata de $A$-módulos so

$$
0 \rightarrow M_{n}^{\prime} \stackrel{\phi_{n}}{\longrightarrow} M_{n} \stackrel{\dot{\psi}_{n}}{\longrightarrow} M_{n}^{\prime \prime} \longrightarrow 0
$$

é uma sequência exata de $A$-módulos, para todo $n$.

Teorema B.1.5: ([13], pag. 362) Seja $\gamma_{1}, \ldots, \gamma_{s}$ elementos do anel $A$ e considere a seguinte sequência exata de complexos de A-módulos.

$$
0 \longrightarrow M^{\prime} \longrightarrow M \longrightarrow M^{\prime \prime} \longrightarrow 0 .
$$

Então os módulos de homologia dos complexos de Koszul de $M, M^{\prime}$ e $M^{\prime \prime}$ com relação a $\gamma_{1}, \ldots, \gamma_{s}$, estão conectados pela sequência exata:

$$
0 \longrightarrow H_{s} K\left(\gamma \mid M^{\prime}\right) \longrightarrow H_{s} K(\gamma \mid M) \longrightarrow H_{s} K\left(\gamma \mid M^{\prime \prime}\right) \cdots \cdots \cdots
$$




$$
\begin{gathered}
H_{\mu} K\left(\gamma \mid M^{\prime}\right) \longrightarrow H_{\mu} K(\gamma \mid M) \longrightarrow H_{\mu} K\left(\gamma \mid M^{\prime \prime}\right) \longrightarrow \\
H_{\mu-1} K\left(\gamma \mid M^{\prime}\right) \longrightarrow H_{\mu} K(\gamma \mid M) \longrightarrow H_{\mu-1} K\left(\gamma \mid M^{\prime \prime}\right) \longrightarrow \cdots \\
H_{\mu-1} K\left(\gamma \mid M^{\prime}\right) \longrightarrow H_{\mu-1} K(\gamma \mid M) \longrightarrow H_{\mu-1} K\left(\gamma \mid M^{\prime \prime}\right) \longrightarrow 0
\end{gathered}
$$

Teorema B.1.6: ([13], pag. 368) Seja $M$ um A-módulo e $\gamma_{1}, \ldots, \gamma_{s}$ elementos do anel $A$. Se $\gamma_{s}$ nầo é um divisor do zero em $M$, cntão temos so seguinte isomorfismo

$$
I_{\mu} K\left(\gamma_{1}, \ldots, \gamma_{s} \mid M\right) \simeq I_{\mu} K\left(\gamma_{1}, \ldots, \gamma_{s} \mid M / \gamma_{s} M\right)
$$

de A-módulos para cada valor de $\mu$.

Vamos provar agora que a característica de Euler-Poincaré $\mathcal{X}\left(\gamma_{1}, \ldots, \gamma_{s} \mid M\right)$ é aditiva.

Teorema B.1.7: Seja

$$
0 \longrightarrow M^{\prime} \rightarrow M \longrightarrow M^{\prime \prime} \longrightarrow 0
$$

uma sequêrucia exata de A-módulos Noetherianos e suponha que cada termo admite $\gamma_{1}, \ldots, \gamma_{s}$ como um sistema de multiplicidade. Funtāo

$$
\mathcal{X}\left(\gamma_{1}, \ldots, \gamma_{s} \mid M\right)=\mathcal{X}\left(\gamma_{1}, \ldots, \gamma_{s} \mid M^{\prime}\right)+\mathcal{X}\left(\gamma_{1}, \ldots, \gamma_{s} \mid M^{\prime \prime}\right)
$$

Demonstração: Pelo Lema B.1.2, todos os módulos na sequência (B.19) têm comprimento finito. Como o comprimento é uma função aditiva, o resultado segne apli(ando $L_{A}$ na sequência (B.19).

Lema B.1.8: ([13], pag. 370) Seja $M$ um $A$-módulo Noetheriano e $\gamma_{1}, \ldots, \gamma_{s}$ um sistema de multiplicidarle em $M$. Se $\gamma_{s}^{m} M=0$, para algum $m>0$, cnlão

$$
\mathcal{X}\left(\gamma_{1}, \ldots, \gamma_{s} \mid M\right)=0
$$

Teorema B.1.9: Seja $M$ um A-módulo Noetheriano e $\gamma_{1} \ldots, \gamma_{s}$ um sistema de multiplicidade em $M$. Entrão

$$
\mathcal{X}\left(\gamma_{1}, \ldots, \gamma_{s} \mid M\right)=e_{A}\left(\gamma_{1}, \ldots, \gamma_{s} \mid M\right)
$$


Demonstração: Faremos a demonstração por indução em s. So s $s=0$, então por (B.13), o resultado vale. Portanto, iremos supor que o teorema já foi provado para sistemas de multiplicidades em $M$, contendo no máximo s - 1 elementos.

'Tome $F^{\prime}=M /\left(0:_{M} \gamma_{s}^{m}\right)$, onde $m$ foi escolhido de modo que $\gamma_{s}$ nào é um divisor do zero con $F$. Fntão aplicando o Teorema B.1. 7 a sequência exatia

$$
0 \longrightarrow\left(0: M \gamma_{s}^{m}\right) \rightarrow M \longrightarrow F \longrightarrow 0
$$

obtemos que

$$
\mathcal{X}\left(\gamma_{1}, \ldots, \gamma_{s} \mid M\right)=\mathcal{X}\left(\gamma_{1}, \ldots, \gamma_{s} \mid F\right)+\mathcal{X}\left(\gamma_{1}, \ldots, \gamma_{s} \mid\left(0:_{M} \gamma_{s}^{m}\right)\right)
$$

c isto se reduz a

$$
\mathcal{X}\left(\gamma_{1}, \ldots, \gamma_{s} \mid M\right)-\mathcal{X}\left(\gamma_{1}, \ldots, \gamma_{s} \mid F\right)
$$

pelo Teorema B.1.8. Dosde que $\gamma_{s}$ nào é um divisor de zero em $F$, o Tcorcma B.1.6, mostra que temos o seguinte isomorfismo

$$
H_{\mu} K\left(\gamma_{1}, \ldots, \gamma_{s-1}, \gamma_{s} \mid F\right) \sim H_{\mu} K\left(\gamma_{1}, \ldots, \gamma_{s} \mid F / \gamma_{s} F\right)
$$

de A-módulos. Segue disto que

$$
\mathcal{X}\left(\gamma_{1}, \ldots, \gamma_{s} \mid F\right)=\mathcal{X}\left(\gamma_{1}, \ldots, \gamma_{s-1} \mid F / \gamma_{s} F\right)
$$

por (B.20).

Por ontro lado. temos

$$
e_{A}\left(\gamma_{1}, \ldots, \gamma_{s}, \gamma_{s} \mid M\right)-\epsilon_{A}\left(\gamma_{1}, \ldots, \gamma_{s-1}, \gamma_{s}, H\right)=\epsilon_{A}\left(\gamma_{1}, \ldots \gamma_{s-1}, \gamma_{s} \mid F / \gamma_{s} F\right) .
$$

pelas propriedades básicas do símbolo da multiplicidade. Finalmente

$$
e_{A}\left(\gamma_{1}, \ldots, \gamma_{s}, \gamma_{s} \mid F / \gamma_{s} F\right)=\mathcal{X}\left(\gamma_{1}, \ldots, \gamma_{s}, 1 \mid F / \gamma_{s} F\right)
$$

pela hipótese indutiva. Assim o teorema está provado. 


\section{B.2 Complexo de Koszul e Multiplicidades Mixtas}

Nosso objetivo é provarmos o Teorema 4.3.1 usando o Complexo de Kostul. Antes de passarmos a prova do teorema, vamos fixar algumas notaçoess que serão ûteis na sua demonstração.

Suponha que $\mathcal{U}$ seja um conjunto de ideais $I_{1}, \ldots, l_{s}$ do ancl $(A, \mathcal{M}, k, d)$ e $R=$ $\left\{r_{1}, \ldots, r_{s}\right\}$ um conjunto de inteiros näo negativos. Entäo $\mathcal{U}^{R}$, denotará o ideal $\mathcal{M}$ primário $I_{1}^{r_{2}} \ldots I_{s}^{r_{s}}$. Porém vamos modificar isto de modo que $r_{1}, \ldots, r_{s}$ possam ser inteiros negativos tomando $I_{1}^{r}=A$. se $r$ for um inteiro negativo. Em seguida, seja $T=\left\{t_{1}, \ldots, t_{s}\right\}$ um corjunto de indeterminadas. Fntão se $r_{1}, \ldots, r_{s}$ ó um conjunto de inteiros, escrevemos $T^{R}$ para denotar o produto $l_{1}^{r_{1}} \cdots t_{s}^{r_{s}}$. Também escrevemos $T$ para o conjunto $\left\{t_{1}^{-1}, \ldots, t_{s}^{-1}\right\}$.

Definimos o anel $R(\mathcal{U})$ como sendo o subanel de $A\left[T, T{ }^{\mathrm{l}}\right]$ consistindo de todas as somas finitas

$$
\sum c_{R} T^{R} c_{R} \in \mathcal{U}^{R}
$$

O proximo teorema mostra a existencia de redução completa de um conjunto $\mathcal{U}-\left\{I_{1}, \ldots, I_{d}\right\}$ de idlais $\mathcal{M}$-primários. Sua prova, além de extensa, não á relevante. para a contimuação do nosso trabalho.

Teorema B.2.1: ([17], pag. 401) Scjam $I_{l}, \ldots, I_{s}$ ideais de $(A, \mathcal{M})$. Eutão existom elementos $x_{i, j}(j=1, \ldots, d)$ de $I_{i}$, para $i=1, \ldots, s$, tais que se

$$
y_{j}=x_{1 j} \ldots x_{s j} \quad(j=1, \ldots, d)
$$

cutão o ideal $b=\left\langle y_{1}, \ldots, y_{d}>\right.$ ć una reduçäo de $I_{1} \cdots I_{s}$

Definição B.2.2: Seja $x_{i j}(j=1, \ldots, d, i=1, \ldots, s)$ um conjunto de elementos do anel $(A, \mathcal{M}, k, d)$ satisfazendo as condições do 'Teorema B.2.1. Então dizemos que esses elementos são uma redução completa de $\mathcal{U}$.

Definição B.2.3: Seja $\mathcal{U}-\left\{I_{1}, \ldots, I_{d}\right\}$ un corjunto de ideajs $\mathcal{M}$-primários do ancl $(A, \mathcal{M}, k, d)$, não necessarriamente distintos. Entào dizemos que um conjunto de elementos $x_{j}(j=1, \ldots, d)$ é uma "joint reduction" do ideal $\mathcal{U}$, se $x_{i} \subset I_{i}$, para cada 
$i_{d}$ e para algum $R-\left\{r_{1}, \ldots, r_{d}\right\}$

$$
\mathcal{U}^{R}=\sum_{i=1}^{d} x_{i} \mathcal{U}^{R_{i}}
$$

onde $R^{i}=\left\{r_{1}, \ldots, r_{i}-1, \ldots, r_{d}\right\}$.

Observe que estamos definindo algo, que nem sabemos que existe. O seguinte resultado nos fornece uma direção para garant ir a existência de uma "joint reduction" de um conjunto finito de ideais $\mathcal{M}$-primários.

Teorema B.2.4: $\operatorname{Sejam}(A, \mathcal{M}, k, d) \circ U=\left\{I_{1}, \ldots, I_{d}\right\}$ um conjunto finito de ideais $\mathcal{M}$-primários năo necessariamente distintos. Fntão o conjunto $x_{t}(i=1, \ldots, d)$, é uma "joint reduction" de $\mathcal{U}$ se, e somente se, o ideal

$$
C=\sum_{i=1}^{d} x_{i} I_{1} \ldots \hat{I}_{i} \ldots I_{d},
$$

é uma redução do ideal $I_{1} \cdots I_{d}$, onde o símbolo $I$, significa que o ideal $I$, não aparece $\sin x_{i} I_{1} \ldots \hat{I}_{i} \ldots I_{t}$.

Demonstração: Vamos escrever a Equação (B.21) por extenso para entendermos melhor. Enttão,

$$
I_{1}^{r_{1}} \cdots I_{s}^{r_{s}}=x_{1} I_{1}^{r_{1} \cdot 1} \ldots I_{s}^{r_{s}}+\ldots+x_{s} I_{1}^{r_{s}} \cdots I_{s}^{r_{s}} \cdot 1 .
$$

Seja $n \geq \max \left\{r_{1}, \ldots, r_{s}\right\}$. cntäo a Equação (4.1) pode ser eserita dla forma

$$
\begin{aligned}
\left(I_{1} \cdots I_{s}\right)^{n} & =I_{1}^{r_{n}} \cdots I_{s}^{n} \\
& =x_{1} I_{1}^{n-1} \cdots I_{s}^{n}+\ldots+x_{s} I_{1}^{n} \cdots I_{s}^{n-1} \\
& =\left(x_{1} I_{2} \cdots I_{s}+x_{2} I_{1} I_{3} \cdots I_{s} \cdots+x_{s} I_{1} \cdots I_{s-1}\right)\left(I_{1}^{n-1} \cdots I_{s}^{n-1}\right) \\
& =\left(x_{1} I_{2} \cdots I_{s}+x_{2} I_{1} I_{3} \cdots I_{s} \ldots+x_{s} I_{1} \cdots I_{s-1}\right)\left(I_{1} \cdots I_{s}\right)^{n !},
\end{aligned}
$$

ou seja, o ideal $\left(x_{1} I_{2} \cdots I_{s}+x_{2} I_{1} I_{3} \cdots I_{s} \ldots+x_{s} I_{1} \cdots I_{s} \quad 1\right)$, ó uma reduçáo do ideal $I_{1} \cdots I_{s}$. 
Reciprocamente, suponha que o ideal $C=\sum_{i=1}^{-d} x_{i} I_{1} \ldots \hat{I}_{i} \ldots I_{d}$, é uma redução do ideal $I_{1} \cdots I_{d}$. Então, existe um intciro positivo $n$ tal que $\left(I_{1} \cdots I_{d}\right)^{n} C^{\dagger}=\left(I_{1} \cdots I_{d}\right)^{n+1}$, ou seja,

$$
\begin{aligned}
\left(I_{1} \cdots I_{d}\right)^{n+1} & =\left(\sum_{i=1}^{d} x_{i} I_{1} \ldots \hat{I}_{i} \ldots I_{d}\right)\left(I_{1} \cdots I_{d}\right)^{n_{2}} \\
& =\sum_{i=1}^{d} x_{i} I_{1}^{n-1} \ldots I_{i}^{n} \ldots I_{d}^{n}
\end{aligned}
$$

ou seja, $x_{1}, \ldots, x_{d}$, é uma "joint reduction" dos ideal $I_{1} \cdots I_{s}$.

Aparentemente, poderíamos ter usado o Teorema B.2.4, para definir a "joint reduction" do conjunto $\mathcal{U}$, de ideais $\mathcal{M}$-primários, mas mesno assim, continuaríamos sem garantir sua cxistência. O próximo teorema, mostra que a existência de uma redução completa de um conjunto de ideais m-primário $I_{1}, \ldots, I_{d}$, garante a existencia de uma "joint reduction" para estes ideais.

Teorema B.2.5: Seja $\left\{x_{i j} i, j-1, \ldots d\right\}$ uma reduçäo completa de $U-\left\{I_{1}, \ldots, I_{d}\right\}$. Seja $i \longrightarrow j(i)$ uma permutação do conjunto $\{1, \ldots, d\}$. Então o conjunto de elementos $x_{i}=x_{i, j(i)}(i=1, \ldots, d)$ é uma "joint reduction" de $\mathcal{U}$.

Demonstraçāo: Sejam $y_{j}=x_{1 j}, \ldots, x_{d j}, j-1, \ldots, d$, onde $b=\left(y_{1}, \ldots, y_{d}\right)$ ć uma redução de $I_{1} \cdots I_{d}$. Considere o ideal

$$
C=\sum_{i=1}^{d} x_{i, j(1)} I_{1} \ldots \hat{I}_{i} \ldots I_{d, j(d)} .
$$

Então para algum inteiro $n>0$ temos

$$
\begin{aligned}
\left(I_{1} \cdots I_{d}\right)^{n+1} & =b\left(I_{1} \cdots I_{d}\right)^{n} \subseteq C\left(I_{1} \cdots I_{d}\right)^{n} \\
& \subseteq\left(I_{1} \cdots I_{d}\right)^{n+1}
\end{aligned}
$$

ou seja, $C$ é uma redução do ideal $\left(I_{1} \cdots I_{d}\right)$. Note que o ideal $b$ está contido no ideal $C$. Portanto pelo Lema B.2.4, $\left\{x_{i j} i, j=1, \ldots, d\right\}$ é uma "joint reduction"de $\left(I_{1} \cdots I_{d}\right)$.

No final deste capítulo, apresentaremos outra forma de garantir a existência de "joint reductions".

O leitor interessado em saber mais propriedades das "joint reduction"pode consultar as referências [17] e [22]. 
Definição B.2.6: Scja $A$ um ancl. Uma filtração $F \mathrm{em} A$ é uma cadeia descendente

$$
A=I_{0} \supset I_{1} \supset I_{2} \supset \cdots
$$

de ideais de $A$ tais que $I_{i} I_{j} \subset I_{i-j}$

Também podemos definir o conceito de filtração para módulos como segue.

Definiçāo B.2.7: Scjam $M$ um $A$-módulo o $I$ um ideal de A. Lina filtração $F \mathrm{~cm}$ $M$ ć uma cadeia descendente

$$
M-M_{0} \supset M_{1} \supset M_{2} \supset \ldots
$$

de submódulos de $M$ tais que $I M_{n} \subset M_{n-1}$. Neste caso dizemos que $F$ é uma I-filtraçầo em $M$.

Exemplo B.2.8: Sejam $A$ um anel, $M$ um $A$-módulo e $I$ um ideal de $A$. Fntão a secquência $\left\{I^{\prime \prime} M\right\}$ ć uma $I$-filtraçào de $M$. chamada de filtraçäo $I$-ádica.

Queremos agora definir uma filtração para $u$ m A-módulo finitamente gerado, nas não apenas em relaçâo a um único ideal do $A$. Para isto, tome $R^{i}$ para denotar o conjunto $\left\{r_{1}, \ldots, r_{i}+1, \ldots, r_{d}\right\}$. onde $R$ é o conjunto $\left\{r_{1}, \ldots, r_{d}\right\}$.

Definição B.2.9: Sejam $V u$ um A-módulo finitamente gerado e $\mathcal{U}=\left\{I_{1}, \ldots, I_{d}\right\}$. $I_{i}$ ideais m-primários $i-1, \ldots, d$. Por uma $\mathcal{U}$-multifiltraçán $F$, entenderemos um conjunto de submódulos $F(R)$ do $M$ definida para todos os conjuntos de $s$ inteiros $\left\{r_{1}, \ldots, r_{s}\right\}$ e satisfazendo as seguintes condiçòs

$$
F(R) \supseteq F\left(R^{i}\right) \supseteq q_{i} F(R)
$$

para todo $r$ e $i=1, \ldots, s$. No caso, $s=1$. temos uma $I$-filtração.

Associaremos com a $\mathcal{U}$-multifiltração $F$ um $R(\mathcal{U})$-módulo graduado $\mathcal{F}$, consistindo de todas as somas finitas

$$
\sum f(R) T^{R} \operatorname{com} f(R) \in M
$$


Definição B.2.10: Dizemos que $F$ é uma $\mathcal{U}$-multifiltração ótima se as seguintes condiçōes forem satisfesitas:

i) $\mathcal{F}$ é um $R(\mathcal{U})$-módulo finitamente gerado;

ii) $M / F(R)$ tem comprimento finito para todo $R$.

Associaremos com uma $\mathcal{U}$-multifiltração ótima $F$ uma sćrie de potências formal em $Z=\left(z_{1}, \ldots, z_{r}\right)$ definida por

$$
P(F, Z)=\sum_{R} L(R) Z^{R}
$$

onde $L(R)=L(M / F(R)), L$ denota a função comprimento e $Z^{R}$ denota o produto $z_{1}^{r_{1}} \ldots z_{s}^{r_{s}}$. Também podemos considerar as series de potências formais

$$
I_{+}(F, Z)=\sum_{R>0} L(R) Z^{R}
$$

onde $R>0$ indica que $r_{i}>0(i=1, \ldots, s)$.

Vamos listar agora algumas séries de potências formais especiais:

i) $E_{i}-\sum_{-\infty}^{\infty} z_{i}^{r}$. Se $I$ é um subconjunto de $\{1, \ldots, s\}$ então $E_{I}=\prod_{i \in I} E_{i} . \Lambda$ gora se $I=\{1, \ldots, s\}$, então escrevemos $E$ para $E_{l}$.

ii) $w_{i}=\sum_{r-10}^{\infty} z_{2}^{r}$. So $I$ é um subconjunto de $\{1, \ldots, s\}$, então $W_{l}-\prod_{i \in I} w_{i}$. So tivcrmos porém $I=\{1, \ldots, s\}$ entâa $W^{1}$ será escrito para $W_{I}$, desde que $W$ será reservado para o conjunto $\left\{w_{1}, \ldots, w_{s}\right\}$, onde $w_{i}=\left(1-z_{i}\right)^{1}$.

Seja $X-\left\{X_{1}, \ldots, X_{d}\right\}$, onde $X_{j}-x_{j} t_{i(j)}$ e $j \rightarrow i(j)$ ó uma função do conjunto $\{1, \ldots, d\}$ no conjunto $\{1, \ldots, s\}$ dependendo do conjunto $X$ o os elementos $x_{1}, \ldots, x_{d}$ são sujoitos as seguintes condições:

i) $x_{j} \in I_{i(j)}$;

ii) o ideal $b=<x_{1}, \ldots, x_{d}>$ é $\mathcal{M}$-primário.

Vamos considerar o complexo de Koszul de $\mathcal{F}$ com relação a $X$.

Para cada subconjunto / de $\{1, \ldots, d\}$ defina um símbolo $u(I)$. Para qualquer inteiro $p, 0 \leq p \leq d$ defina o $R(\mathcal{U})$-módulo graduado $K_{p}(X, F, M)$ consistindo de 
todas as somas finitas

$$
\sum f(I) u(I)
$$

onde $f(I)$ pertence a $\mathcal{F}$ el $/$ percorre todos os subconjuntos que contém $p$ clementos.

Vamos agora definir uma graduação em $K_{p}(X, F, M)$ da seguinte maneira. Primeiro associamos um grau a u(I). Este será

$$
f(I)-\sum_{j=0}^{d} c_{2(j)}
$$

com $e_{i(j)}$ igual a 1 se $i(j)=i$ e zero caso contrário. Se $f(I)$ tem grau $\left(r_{1}, \ldots, r_{d}\right)$ o grau de $f(I) u(I)$ é $\left(r_{1}+\epsilon_{1}, \ldots, r_{d}+\epsilon_{d}\right)$. Agora $K_{p}(X, F, M)$ é considerado como um $R(\mathcal{U})$-móódulo da maneirá óbvia, isto é, é uma soma direta de $\left(\begin{array}{c}d \\ p\end{array}\right)$ cópias de $\mathcal{F}$ com uma mudança de graul.

Resta definir o operador diferenciaçào $D$ ). Se $I=\left\{i_{1}, \ldots, i_{p}\right\}$ está escrito cm ordem ascendente, denote por $i_{k}$ o conjunto obtido de $I$ pela omissão de $i_{k}$. Então defina

$$
D(f(I) u(I))=\sum_{k-0}^{d}(-1)^{k}{ }^{1} X_{i k} f(I) u\left(\hat{I}_{k}\right)
$$

e estenda $D$ por linearidade. A escolha do gran assegura que $D$ preserva graus e além disso, $D^{2}-0$.

Assim temos o seguinte complexo

$$
\left(K(X, F, M): 0 \rightarrow K_{d}(X, F, M) \rightarrow K_{d-1}(X, F, M) \rightarrow \ldots \rightarrow K_{0}(X, F, M) \rightarrow 0\right.
$$

Considere agora os módulos de homologia deste complexo. Hiles sào $R(\mathcal{U})$-módulos finitamente gerados e além disso são graduados e anulados por $\left\{X_{1}, \ldots, X_{d}\right\}$ e portanto pelo ideal $b$. Segue que as componentes homogencas $H_{i}(X, F, M)_{k}$ são todas A-módulos de comprimento finito o assim podemos definir as séries de Euler-Poincaré

$$
\mathcal{X}(X, F, M, Z)=\sum \mathcal{X}(X, F, M, R) Z^{\prime}
$$

$$
\mathcal{X}_{+}(X, F, M, Z)=\sum_{R \geq 0} \mathcal{X}(X, F, M, R) Z^{r}
$$


onde

$$
\mathcal{X}(X, F, M, Z)=\sum_{i=0}^{d}(-1)^{i} L\left(I_{i}(X, F, M)_{R}\right)
$$

No que segue se $R=\left\{r_{1}, \ldots, r_{s}\right\}$ é un conjunto qualquer de inteiros, $W^{R}$ irá denotar o produto $u_{1}^{r_{i}} \ldots u_{s}^{r_{s}}$. Em particular $W^{-R}$ denotará $\left(1-z_{1}\right)^{r_{1}} \ldots\left(1-z_{s}\right)^{r_{s}}$.

Lema B.2.11: (\{17], pag. 406) Seja $h_{i}$ o número de valores de $j$ para os quais $i(j)=i$, isto é, $\sum_{i-1}^{s} h_{i}=d \mathrm{c} \operatorname{seja} H=\left(h_{1}, \ldots, h_{s}\right)$. Então

$$
\mathcal{X}(X, F, M, Z)=e(b, M) E^{\prime}-W^{-H} P(F, Z)
$$

Consideraremos agora uma escolha especial do conjunto $\left\{X_{1}, \ldots, X_{d}\right\}$. Suponhamos que $H=\left(h_{1}, \ldots, h_{s}\right)$ ó dado e selecionemos os primeiros $h_{1}$ elementos $x_{j}$ de $q_{1}$, os próximos $h_{2}$ elementos de $q_{2}$ c assim por diante. Suponha além disso que $\left\{x_{1}, \ldots, x_{d}\right\}$ seja una joint reduction de $\mathcal{U}$. Dizemos neste caso que $\left\{x_{1}, \ldots, x_{d}\right\}$ é uma joint reduction de $\mathcal{U}$ de tipo $I$.

Scja $J$ o conjunto dos inteiros $i$ tais que $h_{i}>0$. Fntão segue da definição de joint. reduction que o ideal $X R(\mathcal{U})$ contém todos os elementos de grau $R$ se $r_{i}$ é suficientcmente grande para todo $i$ pertencente a $J$. Neste caso, seguc que $H_{p}(X, F, M)_{R}=0$ se $r_{i}$ é suficientemente grande para todo $i \in J$ desde que $H_{p}(X, F, M)$ c anulado por $X R(\mathcal{U})$ e por conseqüência $\mathcal{X}(X, F, M, Z)=0$ se $r_{i}$ é suficientemente grande para todo $i \in J$.

Lema B.2.12: ([17, pag.40T) $P_{1}(F, Z)$ pode ser cxpresso na forma $W^{1} h(F, W)$ onde $h(F, W)$ í un polinômio enn $w_{1}, \ldots, w_{s}, w_{1}^{-1}, \ldots, w_{s}{ }^{1}$.

Podemos reduzir o polinômio $h(F, W)$ a sua forma padrão $\sum_{I I} h_{H}(W) W^{H}$. onde $H$ denota um conjunto de inteiros não negativos e $h_{H}(h)$ ó um polinômio envolvendo aqueles $w_{i}$ para os quais $h_{i}-0$. 
Lema B.2.13: ([17]. pag. 407) Sejam $B$ um domínio de integridade c $B_{5}$ o anel dos polinômios em $u_{1}, \ldots, w_{s}, u_{1}{ }^{1}, \ldots, w_{s}^{-1}$. Seja $g$ o homomorfismo do $B_{s}$ no ancl das sćries do potencias formal con coeficientes $\mathrm{cm} B$ nas indeterminadas $z_{1} \ldots \ldots z_{s}$ definido por

$$
g\left(u_{i}\right)-\sum_{i=0}^{\infty} z_{i}^{r}, \quad g\left(w_{i}^{-1}\right)=1-z_{i}
$$

Seja $J$ um subconjunto de $\{1 \ldots, s\}$ e suponha que $G$ é um clemento de $B_{s}$ tal que o coeficiente de $Z^{R} \mathrm{em} g(G)$ seja zero se $r_{i} 6$ suficientemente grande para todo $i \in J$. Então o coeficiente de $W^{H}$ en $F$ é zero se $h_{i}>0$ para todo $I \in J$.

Vamos enunciar o T'orema 4.3 .1 de uma forma mais completa.

Teorema B.2.14: ([17]: Teorema 2.4$)$ Seja $h(F, W)$ tendo o mesmo significado que no Lema B.2.12 e seja $\sum_{\| l} h_{H}(W) W$ "sua forma padrão. Então valem as seguintes atirmaçōss.

i) Se $0=(0, \ldots 0)$, então $h_{0}(W)$ é um polinomio do grau $\leq d$ em $w_{1}, \ldots, w_{s}$;

ii) Se $G=\left\{g_{1}, \ldots, g_{s}\right\}$ é um conjunto de inteiros nào negativos satisfazendo $\sum_{i-1}^{s}=d$, cntão o coeficiente de $W^{(j}$ em $h_{0}(W)$ é igual a $e(b, M)$ para qualquer ideal gerado pelos elementos de uma joint reduction de $I_{1}, \ldots, I_{s}$ de tipo $G$ :

iii) Todos os ideais $b$ gerados pelos elementos de uma joint reduction de $I_{1}, \ldots, I_{s}$ de tipo $G$ tem o mesmo símbolo de multiplicidade $e(b, M)$;

iv) Os termos de grau $d$ em $h_{0}(W)$ dependem apenas de $I_{1} \ldots \ldots I_{s}$ e $M$ c nâo da. multifiltração ótima F' cm consideraçâ:

v) Se $H \neq 0$ então $h_{H}(W)$, graı $<d$.

Demonstração: Faremos a demonstração apenas do item (ii). Para a demonstração de (i) e (v) ver [17] Teorema 2.4. As demonstraçoes de (iii) e (iv) decorrem de (i).

Seja $G=\left(g_{1}, \ldots g_{s}\right)$ qualquer conjunto de inteiros não-negativos tais que $\sum_{i=1}^{s}-$ d a seja $J$ o conjunto de índiecs $i$ tais que $y_{i}>0$. Segue do lema 13.2 .11 o das 
observaçòes que o seguiram que a séric de potèncias

$$
(b, M) W^{1}-W^{-G} P_{-}(F, Z) \text { onde } w_{i}=\sum_{i=0}^{\infty} z_{i}^{r}
$$

tem coeficiente zero para $Z^{k}$ se $r_{i}$ é suficientemente grande para todo $i$ pertencente a $J$. Portanto se aplicarmos o lema B.2.13 ao polinômio

$$
e(b, M) W^{1}-W^{-G} W^{\prime} h(F, W)
$$

vemos que o coeficiente de $W^{H}$ neste polinomio á zero se $h_{i}>0$ para todo $i$ pertencente a $J$. Então desde que o conficiente de $W^{1}$ deve ser zero, segue que o coeficiente de $W^{G}$ em $h_{0}(W)$ ć e $(b, M)$ como queríamos demonstrar.

Vamos enunciar o Teorema B.2.14 no caso de dois ideais.

Teorema B.2.15: Sejam $I_{1}$ e $I_{2}$ dois ideais $m$-primários de $u m$ ancl local do dimensão $d$. Fntão $e_{i}\left(I_{1} \mid I_{2}\right), i=0,1, \ldots, d$ denota a multiplicidade do ideal gerado por qualquer joint reduction do conjunto de ideais que consistem de $d$ - $i$ cópias de $I_{1}$ e de $i$ cópias de $q_{2}$

Demonstração: Caso particular do Teorema B.2.14.

Exemplo B.2.16: Seja $A=\mathbb{C}[x, y, z]$ o anel dos polinomios nas indetcrminadas $x, y, z$ sobre o corpo $\mathbb{C}$ dos números complexos. Seja $I_{1}=\mathcal{M}=\langle x, y, z\rangle \mathrm{e} I_{2}=<$ $x, y^{2}, y \approx, z^{2}>$. Vamos calcular $\epsilon_{i}\left(I_{1} \mid I_{2}\right) ; i=0,1,2,3$.

Desde que $K=\left\langle x, y^{2}, z^{2}>\right.$ é uma redução de $I_{2}$, temos que $e_{i}\left(I_{1} \mid I_{2}\right)=e_{2}\left(I_{1} \mid K\right)$ para todo $i=0,1,2,3$

A equação

$$
\mathcal{M}^{2} K^{2}=y \mathcal{M} K^{2}+z \mathcal{M} K^{2}+x \mathcal{M}^{2} K
$$

mostra que $\{y, z, x\}$ é uma joint reduction do conjunto $\left\{\mathcal{M}, \mathcal{M}, K^{\prime}\right\}$. Analogamente a. equaçäo

$$
\mathcal{M}^{2} K^{2}-y \mathcal{M} K^{2}+z \mathcal{M}{ }^{2} K+z^{2} \mathcal{M}^{2} K
$$

mostra que $\left\{y, x, z^{2}\right\}$ e uma joint reduction do conjunto $\{m, K, K\}$.

$$
\epsilon_{0}\left(I_{1} \mid I_{2}\right)=\epsilon_{0}\left(I_{1} \mid A\right)=e(\mathcal{M} \mid A)=1
$$




$$
\begin{aligned}
& \iota_{1}\left(I_{1} \mid I_{2}\right)-e_{1}(\mathcal{M} \mid K)=e(y, z, x \mid A)=1 \\
& \epsilon_{2}\left(I_{1} \mid I_{2}\right)=e_{2}(\mathcal{M} \mid K)-2 \\
& \epsilon_{3}\left(I_{1} \mid I_{2}\right)-e_{3}\left(I_{2} \mid A\right)=e\left(x, y^{2}, y z, z^{2} \mid A\right)=4
\end{aligned}
$$

O Teorema 4.3.1, aparece originalmente em ([23], pag. 302). () Teorema B.2.14, ć uma generalização do Teorema 4.3.1. Para ver isto, vamos enunciar um lema que nos permitirá definir elementos gerais.

Toda sequência de elementos superficiais forma uma "joint reduction" do conjunto do ideais $\left\{I_{1}, \ldots, I_{1} \ldots, I_{s}, \ldots, I_{s}\right\}$ com relaçäo a $M$, onde cada $I_{i}$ aparece di vezes [[22], pag. 4]. Assim a existência de elementos superficiais garante a existência de "joint reduction" de $d$ idcais m-primários com relação a $M$. Isto fornece a generalização de falamos anteriormente.

() Teorema B.2.14, tem uma espécie de recíproca. Ele envolve outros conceitos näo tratados nesta dissertação, mas vamos incluí-lo mesmo assim. Um anel Voetheriano local é chamado "quasi-unmixed" se seu completamento $A^{*}$ é equidimensional.

Teorema B.2.17: ([22], pag. 12) Sejan $(A, m)$ um anel Noetheriano local "quasiunmixed", $I_{1}, \ldots, I_{s}$ ideais o $a_{i}$ um elemento de $I_{i}$ para cada $i=1, \ldots, s$. Suponha que o radical do idcal $\left(a_{i}, \ldots, a_{s}\right)$ ć o mesmo que o radical de todos os $I$, o que sua altura comum és. So

$$
e\left(\left(a_{i}, \ldots, a_{s}\right) A_{p} ; A_{p}\right)=e\left(I_{1} A_{p}, \ldots, I_{1} A_{p} ; A_{p}\right)
$$

para cada ideal primo minimal de $\left(a_{i}, \ldots, a_{s}\right)$, então $\left(a_{2}, \ldots, a_{s}\right)$ ć uma "joint reduction" de $\left(I_{i}, \ldots, I_{s}\right)$ 


\section{Referências Bibliográficas}

[1] Atiyah, M. F.; Macdonald, I. G. Introduction to commutative algebra. AddisonWosley Publishing Co., Reading, Mass.-London-Don Mills, Ont. 1969.

[2] Auslander. M.; Buchsbaum, D.; Codimension and multiplicity. Ann. of Math. (2) $68(1958) 625-657$.

[3] Bhattacharya, P.; 'The Hilbert functions of two ideals, Math. Proc. Cambridge Philos. Soc. $53(1957) 568-575$.

14] Bruns, W.; Herzog, J.: Cohen-Macaulay rings. Cambridge Studies in Advanced Mathematics, 39. Cambridge Lniversity Press, Cambridge, 1993.

[5] Fernandes, L.; Fecho integral de ideais analíticos e equisingularidades. Dissertação (Mostrado). USP-ICMC. São Carlos, 2001.

[6] Gunning, R.; Introduction to holomorphic functions of several variables. Vol. II. Iocal theory. The Wadsworth and Brooks/Cole Mathematies Series. Wadsworth and Brooks/Cole Advanced Books and Software, Monterey, CA, 1990.

[7] Lech, C.; Note on multiplicities of ideals. Ark. Mat. $4196063-86$.

[8] Lech, C; On the associativity formula for multiplicities. Ark. Mat. 3 (1957), 301-314.

[9] Matsumura,H.; Commutative ring theory, Cambridge Studies in Advanced Mathematics 81988 . 
[10] Nagatia, M.; The theory of multiplicity in general local rings. Procedings of the intenational symposium on algebraic number theory, Tokyo and Nikko, (1955), pp. 191 - 226. Science Council of Japan, Tokyo, 1956.

[11] Northeott, D.; Hilbert's function in a local ring. Quart. J. Math., Oxford Ser. 4 (1953) $67-80$.

[12] Northcott, D.; Ideal theory. Cambridge 'Tracts in Mathematics and Mathematical Physies, No. 42. Cambridge, at the University P'ress, 1953.

[1:3] Northeott, D.; Lessons on rings,modules and multiplicitins. Cambridge University Press, London. 1988.

[14] Northeott, D.; Rees, 1); Reductions of ideals in local rings. Proc. Cambridge Philos. Soc. 57 (1954) $145 \ldots 158$.

[15] Nortbcot1, D. G.: Reufel, M.A.; Generalization of the concept of length Quart. J. Math. Oxford Ser. (2) 16 (1965). $297-321$.

[16] Rees, D.; a-transforms of local rings and a theorem on multiplicities of ideals. Math. proc. Cambridge Philos. Soc. 57(1961) $8-17$.

17] Rees, D.; Generalizations of reductions and mixed multiplicities. J.Lond.Math.Soc. 29,(1984) $397-414$.

[18] Rees, D.; Lectures on the asymptotic theory of ideals. I.ondon Mathematical Society Lecture Note Scries, 113. Cambridge University Press, Cambridge, 1988.

[19] Roberts, Paul C.; Multiplicities and Chern classes in local algebra. Cambridge Tracts in Mathematics, 133. Cambridge University l'ress, Cambridge, 1998.

[20] Samucl, P; La notion de multiplicití en algèbre et en gécmétrie algébrique. (French) J. Math. Pures Appl. (9) 30, (1951). 159-205.

21] Serre, Jean-Pierre: Local algebra. Translated from the French by Cheowhye Chin and revised by the author. Springer Monographs in Mathematics. SpringerVerlag, Berlin, 2000 . 
[22] Swanson, I.; Mixed multiplicities, joint reductions and quasi-tunmixed local rings. J. London Math. Soc.(2) 48 (1993) $1-14$.

[23] Teissicr. B.; Cycles évanescentes, sections planes et conditions of Whitncy, Astérique 7-8,(1973) 285-362

[24] Tomazella, J. N.; Número de Milnor. Dissertação (Mestrado). USP-ICMC. Sao Carlos, 1992.

[25] Trung, N. V.; Positivity of mixed multiplicities. Math. Ann. 319 (2001), no. 1 , 33-63.

[26] Verma,J; Extended Rees algebras and mixed multiplicities. Mathematishe Zeitschrift.202 (1989) $111-128$.

[27! Verma, J. K.; Multigraded Rees algebras and mixed multiplicities. J. Pure Appl. Algebra 77 (1992), no. 2, 219-228.

[28] Verma, J.: Rees algebras and mixed multiplicities.l'roc. of the A.M.S. 104 (1988) $1036-1044$.

[29] Verma, J. K.; Rees algebras with minimal multiplicity. Comm. Algebra 17 (1989), no. $12,2999-3024$.

[30] Viêt, D. Q.; . Mixed multiplicities of arbitrary ideals in local rings. Comm. Algebra. $28(2000)$, no. $8,3803-3821$.

[31] Wright, D. J.; General multiplicity thcory. Proc. London Math. Soc. 15 (1965) $269-288$.

[32] Wright, D. J.; On the uniqueness of a definition of multiplicity,Quart. J. Math. Oxford Ser. (2) 21 1970 171-176.

[33] Zariski,O.and Samuel,P.: Commutalive algebra. Vol. 1. Craduate Texts in Mathcmatics, No. 28. Springer-Verlag, New York-Heidelberg-Berlin, 1975. 
[34] Zariski, O. and Samucl, P.; Commutative algebra. Vol. II. Graduate Texts in Mathematics, Vol. 29. Springer-Verlag, New York-Heidelberg, 1975. 


\title{
Índice Remissivo
}

\author{
altura, 13 \\ ancel \\ Artiniano, 6 \\ Cohen-Macanlay, 15 \\ Nontheriano, 5 \\ '1-graduado, 16 \\ de Rees extendido, 88 \\ de Rees, 88 \\ de fraçòes, 123 \\ dos germess analíticos, 21 \\ extensão, 77 \\ graduado associado, 19 \\ local, 5 \\ primário, 76 \\ anulador. 13 \\ bigraus, 87 \\ radela \\ de ideais primos, 12 \\ primária, 10 \\ coeficientes de Hilbert, 63 \\ complexo \\ de Koszul, 128 \\ de módulos, 127 \\ componente
}

bigraduada, 81

homogênea, 16

primária, 11

comprimento

de cadeia, 12

de módulo, 7

série normal, 7

corpo

residual, 5

decomposição

irredundante, 11

normal, 11

primária, 11

dimensäo

de Krull, 12

de módulo, 13

alemento

M-regular, 14

bihomogêneo, 81

homogèneo, 16

integral sobre anel, 125

inteiro, 100

rilpotente, 7

suficientemente geral, 107 


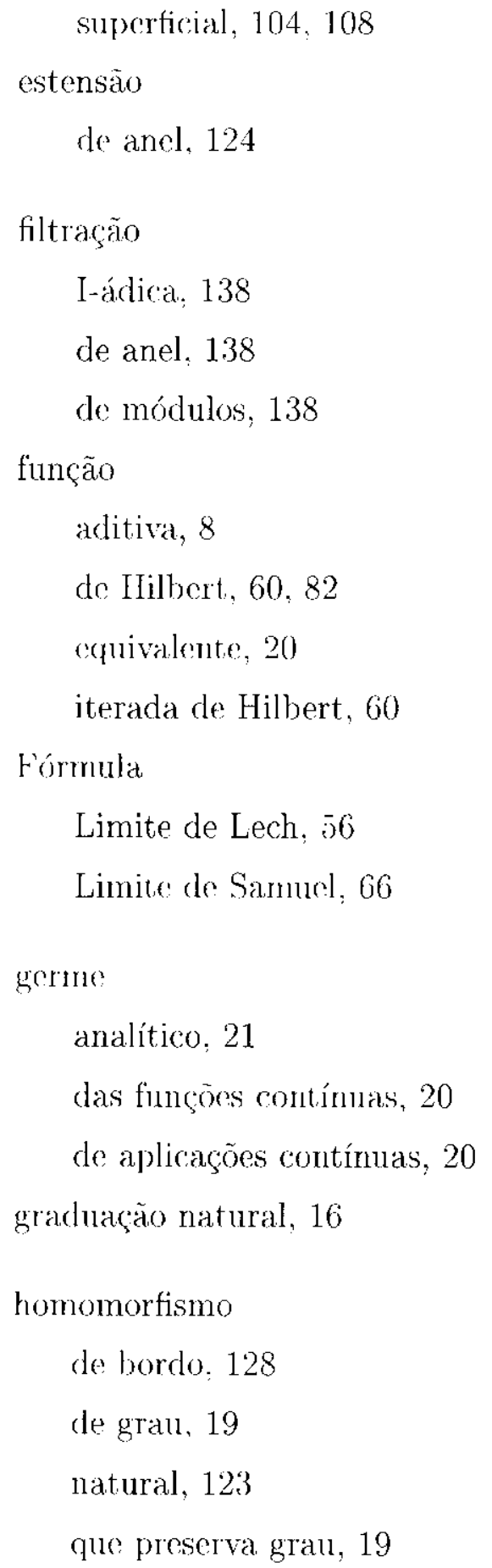

superficial, 104, 108

estensão

de anel, 124

filtração

I-ádica, 138

de anel, 138

de módulos, 138

função

aditiva, 8

de Hilbert, 60, 82

equivalente, 20

iterada de Hilbert, 60

Fórmula

Limite de Lech, 56

Limite: de Samuel, 66

gerine

analítico, 21

das funçoes contínuas, 20

de aplicações contínuas, 20

graduação natural, 16

homomorfismo

de bordo, 128

de grall, 19

natural, 123

que preserva grau, 19

I-filtração, 138

icleal

associado, 12

bihomogeneo, 81 contração, 77

decomponivel, 11

extensâo. 77

m-primário, 9

nilpotente, 7

nilradical, 7

p-primário, 9

parâmetro, 14

primo imerso, 12

primo minimal, 11

primário, 9

projetivamente irrelevante, 85

integralmente fechado, 125

joint reduction, 135

Lei Associativa, 126

localização, 12:3

M-sequência, 14

monóide de graduação, 15

multifiltıração, 138

multifiltração ótima, 139

multipliciclade. 28

multiplicidades mixtas, 95

nódulo

Artiniano, 6

Gohem-Macanlay, 14

Noctheriano, 5

'T-graduado, 17

componente, 128

do IIilbert, 61 
de homologia, 128

graduado associado, 19

n-bordos, 128

n-ciclos: 128

número

de Milnor, 117

número de Milnor, 116

polinômio

bihomogêneo, 81

de Bhattacharya, 95

quasi-homogêneo, 118

ponto

regular, 119

singular, 119

profundidade

de um módulo, 14

radical, 9

radical de Jacobson, 5

redução completa, 135

reduçăo de ideal, 97

singularidade, 119

singularidade isolada, 119

sistema.

de multiplicidades, 24

de parâmetros, 13

submódulo

homogeneo, 18

série

de composicão, 7 normal, 7

T-graduação, 16

incluzida, 18

quociente, 19

T-graduação trivial, 16

Teorema

da Base de Hilbert, 6

topologicamente equivalente, 116 